\title{
Métodos de seleção de pontos de corte em Análise de Sobrevivência
}

\author{
Gisele Cristine Eugenio \\ DiSSERTAÇÃO APRESENTADA \\ $\mathrm{AO}$ \\ Instituto DE MATEmÁtica e EstatísticA \\ $\mathrm{DA}$ \\ Universidade DE SÃo PAUlo \\ PARA \\ OBTENÇÃO DO TÍTULO \\ DE \\ Mestre em CiênCIAS
}

Programa: Estatística

Orientador: Prof. Dr. Antonio Carlos Pedroso de Lima

São Paulo, junho de 2017 


\section{Métodos de seleção de pontos de corte em Análise de Sobrevivência}

Esta versão da dissertação contém as correções e alterações sugeridas pela Comissão Julgadora durante a defesa da versão original do trabalho, realizada em 05/06/2017. Uma cópia da versão original está disponível no

Instituto de Matemática e Estatística da Universidade de São Paulo.

Comissão Julgadora:

- Prof. Dr. Antonio Carlos Pedroso de Lima - IME-USP

- Prof. Dr. Lúcia Pereira Barroso - IME-USP

- Prof. Dr. Liciana Vaz de Arruda Silveira - UNESP-Botucatu 


\section{Agradecimentos}

Primeiramente, quero agradecer ao meu orientador Antonio Carlos Pedroso de Lima os ensinamentos, a disponibilidade e a gentileza nas palavras ao me auxiliar e direcionar pelos melhores caminhos. Certamente, sem o seu apoio e profissionalismo esse trabalho não teria sido realizado com tanto esmero e motivação.

Quero agradecer também aos familiares que me apoiaram de tantas formas e me deram forças para concluir esta etapa, em especial a minha mãe Vanda que me auxiliou a todo momento, minha tia Vania que sempre acreditou em mim e me deu conselhos incríveis, ao meu irmão Leonardo que sempre me motivou e ao meu amigo/irmão que sempre está ao meu lado em todos os momentos, Felipe.

Quero agradecer a todos os amigos que fiz durante o período do mestrado, que estiveram ao meu lado e tornaram meus dias mais leves e felizes, em especial ao Helder que tanto me ouviu e ajudou, ao Renan, Willian (79), Aninha e Giulia. Os dias de jogos, filmes, campings e conversas foram especiais e inesquecíveis. E, também, um agradecimento especial a minha psicóloga e amiga Magali, e a minha professora e amiga Daniela.

Gisele Cristine Eugenio São Paulo, junho de 2015 


\section{Resumo}

EUGEniO, G. C. Métodos de seleção de pontos de corte em Análise de Sobrevivência. 2017. Dissertação - Instituto de Matemática e Estatística, Universidade de São Paulo, São Paulo, 2017.

Este trabalho visa apresentar métodos de categorização de variáveis explicativas contínuas em Análise de Sobrevivência. Do ponto de vista clínico, agrupar pacientes em grupos de risco distintos é importante para agilizar tomadas de decisões; entretanto, perda de informação e outros problemas estatísticos podem ocorrer. Portanto, métodos para seleção de pontos de corte e correção dos possíveis problemas gerados pela categorização são criticamente avaliados. Para a aplicação e comparação dos métodos são utilizados dados do Instituto do Coração do Hospital das Clínicas da Faculdade de Medicina da Universidade de São Paulo (InCor - FMUSP), em que a variável fração de ejeção é dicotomizada e tricotomizada.

Palavras-chave: seleção de pontos de corte, método do valor- $p$ mínimo, método da diferença das verossimilhanças, modelo de Cox 


\section{Abstract}

EUGENIO, G. C. Cutpoints selection methods in Survival Analysis. 2017. Dissertação - Instituto de Matemática e Estatística, Universidade de São Paulo, São Paulo, 2017.

This dissertation aims to present methods of categorization for continuous variables in Survival Analysis. From a clinical point of view, grouping patients into distinct risk groups is important for accelerating decision-making; however, loss of information and other statistical problems may occur. Therefore, methods for selecting cutpoints and correcting problems generated by categorization are critically evaluated. For the application and comparison of the methods, the dataset from Heart Institute - University of Sao Paulo Medical School (InCor - FMUSP) is used, in which the variable ejection fraction is dichotomized and trichotomized.

Keywords: cutpoint selection, minimum $p$-value method, difference of the likelihoods method, Cox model 


\section{Sumário}

1 Introdução 1

2 Motivação $\quad 5$

3 Categorização de variáveis independentes em análise de sobrevivência 11

3.1 Problemas oriundos da categorização de variáveis contínuas . . . . . . . . . . 12

3.2 Métodos descritivos de categorização . . . . . . . . . . . . . . . . . . . . 14

3.3 Métodos de seleção de ponto de corte . . . . . . . . . . . . . . . 18

3.3.1 Método do valor- $p$ mínimo . . . . . . . . . . . . . . . 19

3.3.2 Método da diferença das verossimilhanças . . . . . . . . . . . . 25

3.3 .3 Testes corrigidos . . . . . . . . . . . . . . . 26

3.3 .4 Outros métodos . . . . . . . . . . . . . . . . . . . 31

4 Métodos de validação de pontos de corte e de correção do valor- $p$ e do risco relativo $\quad 35$

4.1 Validação de resultados . . . . . . . . . . . . . . . . . . . 35

4.2 Correções para o valor-p no caso de dicotomia . . . . . . . . . . . . . . 36

4.2.1 Método de Lausen92 . . . . . . . . . . . . . . . . . . . . . . . 36

4.2 .2 Método de Lausen94 . . . . . . . . . . . . . . . . . . . . . . 38

4.2.3 Outras correções . . . . . . . . . . . . . . . . . . 39

4.3 Correções para o risco relativo no caso de dicotomia . . . . . . . . . . . . . . 40

4.3 .1 Fator de contração ad hoc . . . . . . . . . . . . . . . . . . 40

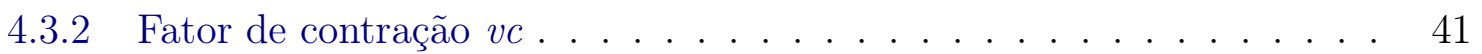

4.3.3 Fator de contração boot . . . . . . . . . . . . . . . . . . 42

4.3 .4 Fator de contração heurístico . . . . . . . . . . . . . . . . . 42

4.4 Correção para valor-p e razão de risco . . . . . . . . . . . . . . . . . . 42

4.4 Validação cruzada . . . . . . . . . . . . . . . . 43

4.4 .2 Split-Sample . . . . . . . . . . . . . . . . . . . . . . 44

5 Aplicação $\quad \mathbf{4 5}$

5.1 Análise gráfica . . . . . . . . . . . . . . . . . . . . . 49

5.2 Resultados para dicotomia . . . . . . . . . . . . . . . . . . 50

5.2 .1 Correção dos resultados . . . . . . . . . . . . . . . 53 
5.3 Resultados para tricotomia . . . . . . . . . . . . . . . . . . 54

5.3 .1 Correção dos resultados . . . . . . . . . . . . . . . . 55

5.4 Validação dos pontos de corte, valores- $p$ e riscos relativos . . . . . . . . . . 56

5.5 Comparação dos métodos . . . . . . . . . . . . . . . . . . . 59

5.5 .1 Análise gráfica . . . . . . . . . . . . . . . . . . 59

5.5.2 Resultados para dicotomia . . . . . . . . . . . . . . 60

5.5.3 Resultados para tricotomia . . . . . . . . . . . . 63

5.5.4 Validação dos pontos de corte, valores- $p$ e riscos relativos . . . . . . 64

5.6 Modelos finais . . . . . . . . . . . . . . . . . . . . 66

5.7 Discussão . . . . . . . . . . . . . . . . . . . . . . 71

5.7 .1 Pesquisas futuras . . . . . . . . . . . . . . . 73

A Testes da razão de verossimilhanças, Wald e Score $\quad 75$

$\begin{array}{ll}\text { B Outras definições } & 79\end{array}$

C Tabelas para os resultados dos métodos de correção e validação $\quad 81$

D Histogramas da distribuição dos pontos de corte e riscos relativos estima$\begin{array}{ll}\text { dos pelo método boot } & 95\end{array}$

$\begin{array}{ll}\text { Referências Bibliográficas } & 111\end{array}$ 


\section{Capítulo 1}

\section{Introdução}

Em pesquisas médicas, a análise de regressão é usada para desenvolver modelos que contribuam com a predição da resposta de um paciente a partir de uma ou mais variáveis explicativas, tais como fatores prognósticos, fatores de tratamento e características dos indivíduos. Para dados de sobrevivência, o principal objetivo da modelagem de um conjunto de dados é a análise do tempo até a ocorrência de um determinado evento, usualmente o óbito de um paciente, ou a recidiva de uma doença ou de algum sintoma. Nesse contexto, a importância da modelagem e predição da variável resposta está em definir o tratamento, o prognóstico e outras decisões clínicas para cada paciente individualmente e, também, em um processo de triagem, identificar grupos de risco para estratificar os indivíduos por grau de gravidade da doença. Em muitos casos a variável explicativa é medida em escala contínua e a interpretação de sua relação com a variável resposta pode ser difícil para profissionais não habituados à linguagem estatística. Uma das formas para esquivar-se dessa dificuldade é a categorização da variável contínua e a consequente divisão dos pacientes em grupos com características mais homogêneas (Altman e Royston (2000), Colosimo e Giolo (2006) e Tunes-da-Silva e Klein (2011)).

Nos casos em que a formação de grupos é requerida, nem sempre é fácil a identificação dos valores da variável que deveriam ser considerados como divisores dos grupos de pacientes que possuem riscos distintos. Para o caso de pacientes com câncer de mama, por exemplo, o risco de morte é uma função crescente da variável contínua tamanho do tumor, entretanto, essa informação pode não ser esclarecedora no direcionamento de pacientes para diferentes formas de tratamento, fazendo-se útil a definição de grupos para avaliar se apenas uma cirurgia é suficiente, ou se radioterapia ou quimioterapia devem ser associadas ao tratamento. É usual o valor de 2 centímetros para classificar os pacientes com câncer de mama nos grupos de alto e baixo riscos de morte (Mazumdar e Glassman, 2000). Como segundo exemplo, o estudo SABE buscou traçar o perfil das pessoas idosas sedentárias do Município de São Paulo e verificar o impacto do sedentarismo na incidência de doenças crônicas, incapacidade e na ocorrência de óbitos entre os idosos; uma das variáveis avaliadas foi o Índice de Massa Corpórea (IMC). Os idosos com baixo peso (IMC $<23 \mathrm{~kg} / \mathrm{m}^{2}$ ) e obesidade (IMC $>30 \mathrm{~kg} / \mathrm{m}^{2}$ ) apresentaram maior tendência ao sedentarismo e, portanto, mais suscetíveis a incidência de incapacidade e a ocorrência de óbito (Paes et al., 2008). 
Dado o uso extensivo da prática de categorização de variáveis contínuas, é importante desenvolver metodologias para seleção dos valores da variável explicativa que dividirão os pacientes em grupos ou categorias com características semelhantes, chamados de pontos de corte ou parâmetro limiar. O modelo ajustado na presença da variável categorizada por meio desses pontos de corte é denotado por modelo categorizado ou modelo com limiar (Klein e Wu (2004) e Bennette e Vickers (2012)). A seleção de pontos para divisão das observações da amostra em dois (dicotomia), três (tricotomia) ou mais grupos (politomia) está relacionado a vários problemas e não há consenso na literatura sobre a melhor estratégia a ser tomada.

Das formas de categorização, a prática de dicotomizar covariáveis originalmente contínuas é a mais comum em pesquisas clínicas, enquanto que no cenário epidemiológico é preferível a divisão em três ou mais grupos para investigação de uma possível relação doseresposta (Royston et al., 2006). Dos pontos de vista clínico e estatístico, as variáveis categóricas são preferidas, pois (Mazumdar et al. (2003), Altman (2005), Royston et al. (2006) e Williams et al. (2006)):

i. No caso de dicotomia, fornecem uma classificação simples em grupos de baixo e alto riscos;

ii. Estabelecem um critério padrão para comparação com estudos subsequentes;

iii. Auxiliam na recomendação de tratamentos terapêuticos;

iv. Definem um critério para o diagnóstico de uma enfermidade;

v. Estimam prognósticos;

vi. Oferecem uma interpretação mais simples de modelos estatísticos comuns como, por exemplo, as medidas razão de chances ou risco relativo;

vii. Evitam a suposição de linearidade implícita em alguns modelos estatísticos para variáveis contínuas e

viii. Fazem a sumarização dos dados mais eficientemente.

A categorização de variáveis contínuas também é útil na definição de um critério padrão para seleção de indivíduos que podem fazer parte de pesquisas para novos tratamentos; como em estudos de câncer, por exemplo, que tendem a excluir pacientes acima de 60 anos, pois acredita-se que esta é a idade que distingue melhor os pacientes com maior ou menor sensibilidade ao tratamento. Outra função importante dessa prática é o auxílio na estratificação de pacientes em testes clínicos, em que os indivíduos devem ser separados em grupos de mesmo tamanho e riscos distintos para aplicação das diferentes formas de tratamento em cada grupo (Mazumdar e Glassman, 2000).

Os métodos de seleção de pontos de corte são divididos em duas categorias: métodos orientados pelos dados e métodos orientados pela resposta (Klein e Wu, 2004). Os métodos 
orientados pelos dados baseiam a escolha do ponto de corte na distribuição da covariável como, por exemplo, o uso da média ou dos percentis. Essa abordagem encontra valores arbitrários e pode não ser útil para verificação do melhor valor para ponto de corte devida a alta dependência da amostra. A segunda abordagem, orientada pela resposta, propõe métodos que baseiam-se na relação entre a covariável e a resposta e seleciona pontos de corte para os quais a covariável categorizada tem o maior efeito sobre a variável dependente. Os pontos de corte encontrados por essa última abordagem são chamados de pontos de corte "ótimos" (Altman e Royston, 2000). Embora os métodos baseados na resposta sejam mais indicados para estimação dos pontos de corte, eles geram uma série de problemas que inspiram cuidados na hora de categorizar variáveis contínuas (Faraggi e Simon (1996) e Mazumdar e Glassman (2000)).

Apesar da utilidade da categorização, a forma como é feita a divisão dos indivíduos pode acarretar problemas graves, tais como: grande perda de informação, redução do poder de detectar a verdadeira relação entre as variáveis explicativa e resposta, variáveis importantes podem aparentar ser não significativas e a quantidade de grupos escolhida pode não ser adequada para indicar os diferentes grupos de risco. Para diminuir tais efeitos e reduzir a chance da categorização conduzir a um modelo clinicamente ou estatisticamente não significativo é importante procurar métodos de seleção de pontos de corte adequados ao banco de dados, utilizar técnicas de correção do possível viés dos parâmetros estimados ocasionado pelos problemas intrínsecos à categorização e métodos de validação dos resultados para que eles sejam úteis em pesquisas baseadas em outras amostras (Hilsenbeck e Clark, 1996).

Há uma extensa literatura a respeito da categorização de uma variável contínua em dois grupos, enquanto que pouco é discutido sobre três ou mais. A dificuldade em encontrar métodos para politomia aumenta para dados de sobrevivência, e o quadro piora na presença de censura. Este trabalho visa não só apresentar métodos de seleção de pontos de corte para dados de sobrevivência, mas também discorrer sobre métodos para correção e validação dos valores estimados a partir de um modelo categorizado. Além disso, a extensão para o caso de politomia de alguns métodos outrora aplicados somente em caso de dicotomia é exibida.

A aplicação das metodologias discutidas é feita nos dados de pacientes com insuficiência cardíaca congestiva que fazem acompanhamento pelo Instituto do Coração de São Paulo (InCor). A variável clinicamente relacionada ao óbito, fração de ejeção do ventrículo esquerdo, é alvo da categorização.

O Capítulo 2 exibe a motivação do trabalho, descreve o banco de dados disponibilizado pelo InCor e, também, apresenta uma breve análise sobre a relação da fração de ejeção e o óbito dos pacientes. As metodologias referentes à seleção dos pontos de corte estão apresentadas no Capítulo 3 e os métodos de correção dos parâmetros estimados a partir do modelo com limiar são apresentados no Capítulo 4. A parte de aplicação e comparação dos métodos de seleção de pontes de corte e correção dos parâmetros estão no Capítulo 5 . Todos os gráficos e resultados apresentados foram obtidos por meio do software estatístico $R$. 


\section{Capítulo 2}

\section{Motivação}

A insuficiência cardíaca (IC), também chamada de insuficiência cardíaca congestiva, é uma síndrome clínica complexa que leva a mudanças no tamanho, forma e função cardíaca, prejudicando a capacidade do coração em bombear sangue suficiente para todos os órgãos do corpo (Lala et al., 2016). Múltiplos fatores de risco estão presentes ao mesmo tempo e antecedem o aparecimento da IC, complicando a identificação da gênese da doença e a definição de ações específicas de planejamento de saúde para redução de sua incidência (Freitas et al. (2005) e Nogueira et al. (2010)). Estudos mais intensivos em relação a esta síndrome têm sido feitos e um fator bastante relevante comumente usado para categorizar pacientes em grupos de riscos distintos é a fração de ejeção do ventrículo esquerdo (FEVE ou FE). A FE representa a porcentagem de sangue bombeado para fora do ventrículo esquerdo a cada contração do coração e é definida por:

$$
\mathrm{FE}=\frac{\text { Volume diastólico final - Volume sistólico final }}{\text { Volume diastólico final }} \times 100 \% \text {. }
$$

Segundo a Diretriz Brasileira de Insuficiência Cardíaca Crônica (Bocchi et al., 2012), a forma clássica de divisão de pacientes com esta síndrome é feita segundo os que possuem FE preservada (ICFEP) e os que possuem FE reduzida (ICFER), mais conhecida como IC sistólica. Ainda não existe uma padronização para o valor da FE na definição desses dois grupos. Na literatura, os valores encontrados variam frequentemente entre $40 \%$ e $50 \%$ (Meijers et al. (2016), Bovitz et al. (2016), Messias et al. (2016) e Bocchi et al. (2012)).

Os pacientes com ICFEP e ICFER possuem características distintas em relação à fisiopatologia, diagnóstico e tratamento, e essa dicotomia tem sido questionada por muitos autores, pois alguns médicos e pesquisadores acreditam se tratar da mesma doença com diferentes fenótipos de apresentação; e outros, que se trata de duas entidades distintas (Bocchi et al., 2012). O que tem sido notado nos últimos anos é que a sobrevida de pacientes com FE reduzida aumentou, enquanto o mesmo não pôde ser observado para pacientes com FE preservada, embora esta última tenha sido vista como uma síndrome de menor gravidade e, portanto, alvo de pouca atenção nos últimos anos (El Aouar et al., 2013). Alguns pesquisadores creditam esse dado ao fato da abordagem e o tratamento de pacientes com ICFEP serem focados em aliviar os sintomas e tratar as comorbidades associadas, deixando de lado 
uma investigação mais profunda para o tratamento da insuficiência cardíaca em si. A III Diretriz Brasileira de Insuficiência Cardíaca Crônica, afirma que a ICFEP permanece sem receber a devida atenção e seu tratamento ainda é decidido de forma empírica, mesmo sendo o diagnóstico de metade dos pacientes que sofrem de IC no Brasil (Messias et al. (2016) e Bocchi et al. (2009)).

Grandes avanços terapêuticos no tratamento da IC, principalmente para o grupo de pacientes com ICFER, e de outras enfermidades associadas ao coração estão relacionados com a maior sobrevida dos pacientes e um aumento da prevalência de casos da doença pelo mundo. Estudos estimam que existem 23 milhões de pessoas no mundo que sofrem dessa doença, e que 2 milhões de casos novos são diagnosticados anualmente. Segundo dados do DATASUS, há cerca de 2 milhões de pacientes com IC no Brasil, e uma incidência anual de 240 mil casos. Por consequência, a prevalência de internações hospitalares e de gastos com medicamentos também aumentaram, e a situação se intensifica em países nos quais a população idosa é emergente. As projeções indicam que em 2025 o Brasil terá a sexta maior população de idosos do mundo, com aproximadamente 30 milhões de pessoas, o que deve provocar um grande impacto econômico para o país, principalmente associado às internações (Nogueira et al., 2010).

Mesmo com o aprimoramento do prognóstico e dos tratamentos médicos para insuficiência cardíaca, a IC ainda está associada às principais causas de internação pelo Sistema Único de Saúde (SUS) em pacientes acima de 65 anos, sendo a terceira principal causa de internação entre todas as enfermidades e a maior causa entre as doenças cardiovasculares (Moutinho et al., 2008). Além disso, a hospitalização de pacientes mais graves ainda é frequente e a taxa de sobrevivência é pequena, o que aumenta gastos públicos com internações e procedimentos terapêuticos, indicando uma real necessidade da elaboração de novas estratégias para o controle desses pacientes (Villacorta e Maisel, 2016).

Em maio de 2016, a Sociedade Europeia de Cardiologia (ESC) renovou suas diretrizes para o diagnóstico e tratamento da insuficiência cardíaca, trazendo como uma das principais novidades a inclusão de uma nova subcategoria de classificação na FEVE. Segundo a ESC, deve haver uma subcategoria mediana entre ICFER e ICFEP, chamada de limítrofe. Dividindo, assim, os pacientes em três grupos: IC com FE reduzida (FE $\leq 40 \%)$, IC com FE limítrofe (FE entre 40\% e 50\%) e IC com FE preservada (FE>50\%). Assim como discutido nas diretrizes brasileiras de IC, as diretrizes europeias anteriores também já haviam questionado sobre a divisão clássica em ICFER e ICFEP. Apenas na ICFER as terapias têm se mostrado eficientes sobre a redução da mortalidade. A ESC espera que a identificação da IC com FE limítrofe (ICFEL) como uma nova categoria estimule pesquisas sobre as particularidades deste grupo de pacientes (Santos e Vieira, 2016).

É notável a importância social do tratamento adequado da IC, tanto pela melhora das condições de vida da população, quanto em questões financeiras devido aos gastos públicos gerados pelo tratamento e acompanhamento da doença. Pesquisadores do Instituto do Coração do Hospital das Clínicas da Faculdade de Medicina da Universidade de São Paulo (InCor 
- FMUSP) também desejam dividir e avaliar o tempo de sobrevivência de pacientes com IC de acordo com a fração de ejeção dividida em três subgrupos: reduzida, limítrofe e preservada (Lima e Saito, 2015). Mesmo não havendo fortes evidências para seleção, inicialmente, eles propuseram os pontos de corte para a definição dos grupos baseando-se em suas vivências clínicas como sendo $45 \%$ e 55\%, ou seja, o grupo de pacientes com fração de ejeção reduzida (ICFER) possui valores da variável inferiores ou iguais a 45\%, o grupo com FE limítrofe (ICFEL) possui valores entre $45 \%$ e $55 \%$, ao passo que o grupo com FE preservada (ICFEP) possui valores acima de 55\%. Dada a arbitrariedade da escolha dos pontos, os pesquisadores desejam encontrar pontos de corte confiáveis por meio de métodos estatísticos. Espera-se assim encontrar valores da fração de ejeção para divisão dos pacientes de modo a facilitar o prognóstico e a escolha do tratamento terapêutico mais adequado para cada grupo; além disso, espera-se que os resultados possam ser aplicados a outros bancos de dados em estudos posteriores.

Para auxiliar nesta busca foram avaliados aproximadamente 3000 pacientes do Sistema Único de Saúde (SUS) acompanhados na Unidade Clínica de Ambulatório Geral do InCor no período de julho de 2003 a março de 2014 até o óbito ou a perda de contato. Os pacientes foram avaliados em consulta inicial, em que foram coletadas as informações demográficas e clínicas; as informações laboratoriais e da morfologia do coração foram retiradas de exames prévios, caso disponíveis, ou foram agendados exames, e receberam o diagnóstico de IC e a orientação para o tratamento medicamentoso e outros tratamentos porventura indicados. Todas as formas de obtenção dos dados foram consideradas como se tivessem sido coletadas na consulta inicial. A FEVE foi calculada a partir de parâmetros determinados por meio do ecocardiograma transtorácico e foi inserida diretamente no banco de dados, por isso não foram disponibilizados os parâmetros utilizados para o seu cálculo, volume diastólico final e volume sistólico final. AS informações coletadas foram previamente analisadas no Centro de Estatística Aplicada do Instituto de Matemática e Estatística da USP (Lima e Saito, 2015).

Pacientes que não possuíam a informação sobre a fração de ejeção foram retirados da análise, restando 2370 observações. Além da fração de ejeção, mais 32 possíveis variáveis pronósticas foram coletadas e avaliadas na seleção do modelo adequado para a predição do tempo de sobrevivência de pacientes com IC. A idade dos pacientes variou de 13 a 91 anos; 1361 eram homens, 1009 eram mulheres. As etiologias das cardiopatias foram: cardiopatia hipertensiva em 984 (41\%) pacientes, cardiopatia isquêmica em 428 (18\%), a cardiopatia da Doença de Chagas em 252 (11\%), cardiomiopatia alcoólica em 19 (0.8\%) outras etiologias em 678 (28.8\%). A cardiomiopatia dilatada sem etiologia diagnosticada em 9 (0.4\%) pacientes. O evento considerado como principal resposta do estudo foi o tempo até a ocorrência de óbito por qualquer causa e as seguintes informações foram utilizadas para sua definição:

- Data de entrada no protocolo, definida como a data da primeira consulta.

- Data do último retorno.

- Data de óbito, se ocorrido. 
A variável representante do tempo, em meses, foi calculada pela diferença entre as datas de último retorno (se não houve óbito registrado) ou data de óbito e a data de entrada no protocolo. O tempo foi calculado através da expressão:

$$
\text { tempo }=\frac{\text { data do último retorno ou óbito }- \text { data de entrada no protocolo }}{365.25 / 12} .
$$

A variável indicadora de falha registra 1 em caso de óbito observado e 0 caso tenha havido perda de acompanhamento sem a informação do óbito ou o paciente permanecia vivo no instante de seleção dos pacientes para o conjunto de dados.

Uma característica importante do banco disponibilizado é o grande número de dados faltantes para a maior parte das variáveis, havendo grande quantidade de óbitos observados dentre os pacientes que possuem um ou mais valores de variáveis faltantes. Categorizar as variáveis quantitativas, atribuindo uma categoria chamada "sem resposta", foi um meio de evitar possíveis vícios durante a análise e impedir que outras informações, se presentes para um paciente, fossem desconsideradas nas análises. Os pesquisadores forneceram os pontos de corte para a definição das classes de cada umas das covariáveis contínuas disponíveis. Pacientes com valor igual a zero para as variáveis PAS (pressão arterial sistólica), PAD (pressão arterial diastólica) e diâmetro do ventrículo direito foram considerados como parte da categoria "sem resposta".

Tendo em vista que a fração de ejeção é a principal variável prognóstica em análise deste trabalho, primeiramente foi feita uma avaliação descritiva univariada da FE em relação ao tempo de sobrevivência dos pacientes. O gráfico de dispersão apresentado na Figura 2.1 indica uma nuvem de pontos referente às falhas (pontos pretos) mais densa no canto esquerdo inferior do gráfico, ou seja, para os valores baixos da fração de ejeção (aproximadamente abaixo de 40), tendendo a uma leve diminuição para os tempos maiores de sobrevivência. A forma do gráfico é controlada pela associação entre a FE e o tempo de sobrevivência e pela informação de que a distribuição desta última tende a ser assimétrica para a direita; observa-se menos falhas para valores mais altos da fração de ejeção. Para valores mais altos da FE (aproximadamente acima do 40) ainda é possível observar uma quantidade razoável e uniforme de óbitos estendendo-se pelos valores da FE. A menor concentração de falhas está entre os últimos instantes de tempo.

Para avaliar os pontos de corte propostos pelos pesquisadores, as curvas de Kaplan-Meier para as três categorias da fração de ejeção definidas por tais pontos estão representadas na Figura 2.2. Além disso, foram realizados dois testes não-paramétricos, logrank e Wilcoxon, que sob a hipótese de igualdade das três curvas de sobrevivência, seguem uma distribuição qui-quadrado com dois graus de liberdade. As estatísticas resultaram em 36.9 e 38.8, como visto na Tabela 2.1, com os correspondentes valores- $p$ inferiores a 0.0001 , respectivamente, o que indica a existência de diferenças entre os grupos.

Pelo gráfico é possível perceber que a curva de sobrevivência dos pacientes com ICFER está mais distante das demais e apresenta menor estimativa para as probabilidades de sobre- 


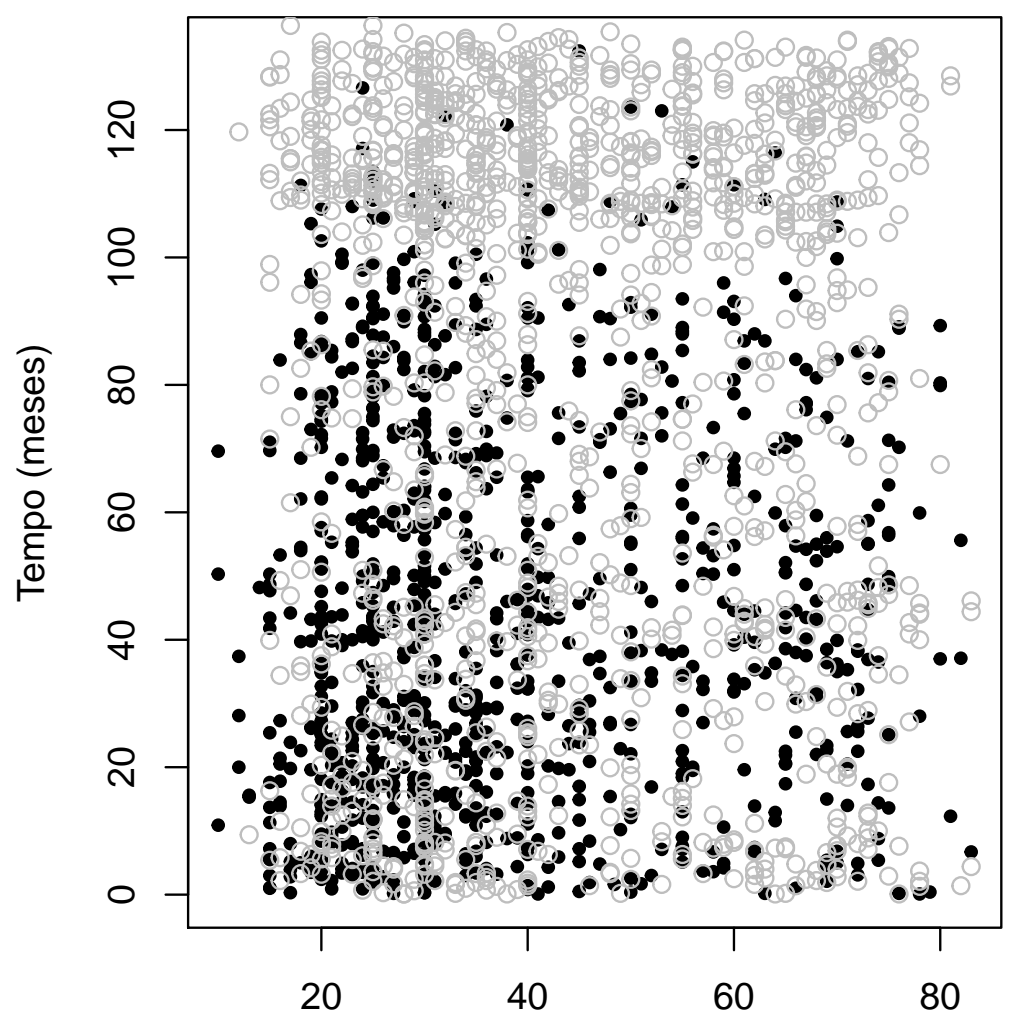

Fração de ejeção (\%)

Figura 2.1: Gráfico de dispersão do Tempo pela FE. Pontos pretos representam os óbitos e os pontos cinzas representam a censura.

vivência para esses indivíduos, enquanto que as curvas para os demais grupos apresentam-se próximas e entrelaçam-se em alguns valores centrais. Uma forma de encontrar as diferenças entre os grupos é comparar os grupos, dois a dois, controlando o erro do tipo I pelo método de Bonferroni padrão. Como existem três grupos, três testes serão necessários e o método de Bonferroni utiliza um nível de significância de $0.05 / 3=0.017$ para cada um dos testes de modo a garantir um nível global de no máximo 0.05 .

Pela Tabela 2.1, como já era notado por meio das curvas de Kaplan-Meier, pode-se concluir que existem diferenças significativas entre os grupos ICFER e ICFEL e entre os grupos ICFER e ICFEP. Entre os grupos ICFEL e ICFEP não há evidências de diferenças. Verifica-se, então, que os pontos de corte não estão sendo capazes de separar em categorias com diferença estatisticamente significante.

O principal objetivo deste trabalho é apresentar métodos para encontrar pontos de corte que limitam de forma mais eficiente os grupos de pacientes que pertencem a cada uma das três categorias da FE. Além disso, métodos para validação dos pontos de corte, razão de risco e nível descritivo dos testes que verificam as diferenças entre os grupos são avaliados. 


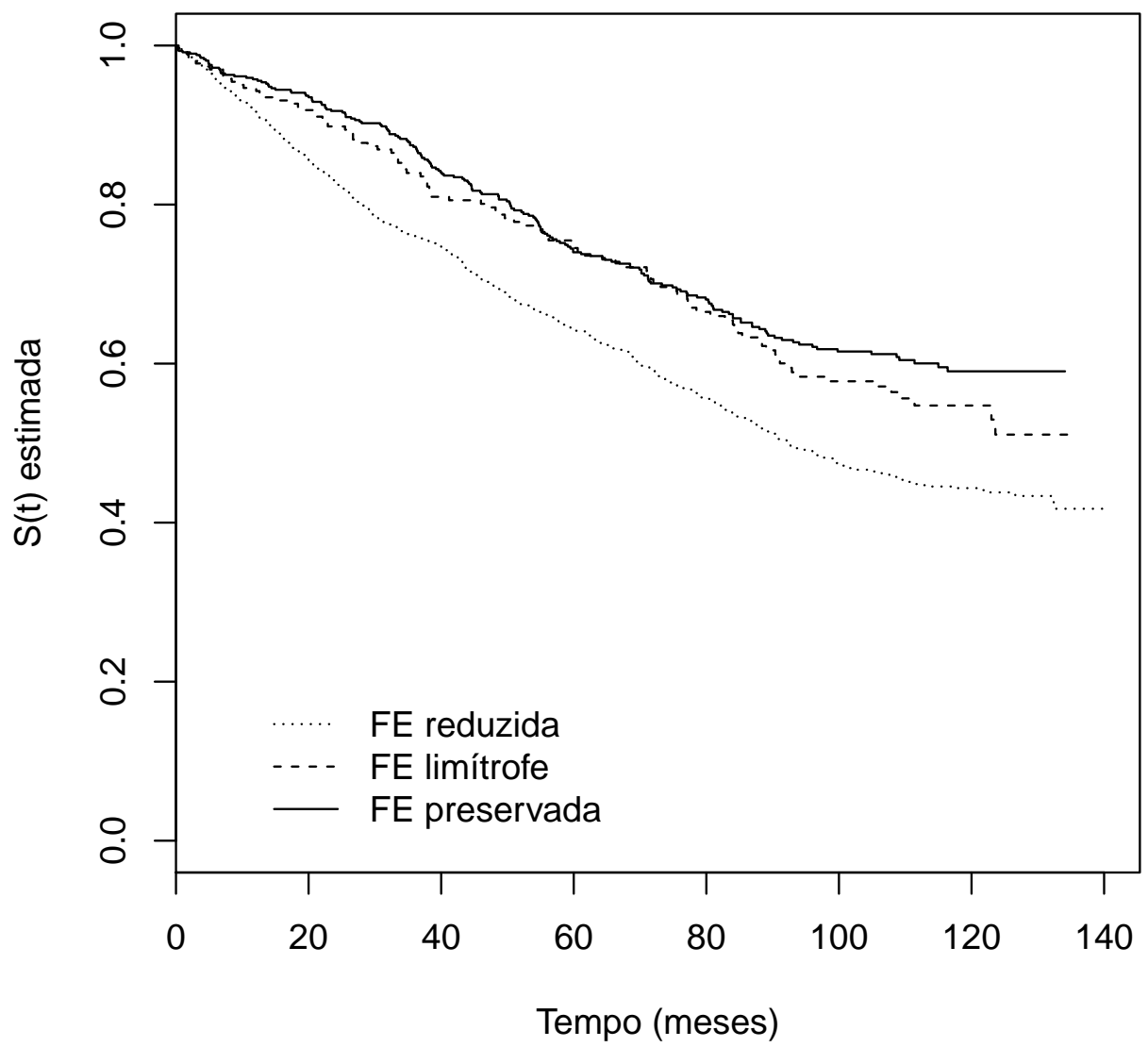

Figura 2.2: Curvas de Kaplan-Meier para as três categorias de FE definidas inicialmente pelos pesquisadores do InCor, com os pontos de corte $45 \%$ e $55 \%$.

Tabela 2.1: Resultados dos testes logrank e Wilcoxon utilizados para as comparações dos grupos de pacientes com ICFER, ICFEL e ICFEP, dois a dois.

\begin{tabular}{ccccc} 
Grupos comparados & Teste logrank & Valor p & Teste Wilcoxon & Valor p \\
\hline Todos & 36.9 & $<0.0001$ & 38.8 & $<0.0001$ \\
ICFER e ICFEL & 9.3 & 0.0023 & 10.5 & 0.0012 \\
ICFEL e ICFEP & 1.2 & 0.2660 & 1.0 & 0.3120 \\
ICFER e ICFEP & 32.0 & $<0.0001$ & 33.1 & $<0.0001$ \\
\hline
\end{tabular}




\section{Capítulo 3}

\section{Categorização de variáveis independentes em aná- lise de sobrevivência}

Os métodos abordados neste capítulo são aplicados em dados de sobrevivência, mais especificamente, no modelo de regressão de Cox. Neste contexto, suponha que o tempo até a ocorrência de um evento de interesse é representado por uma variável aleatória $T$, caracterizada por uma função de sobrevivência $S(t)=P(T>t)$ e uma função de risco $h(t)=$ $-d(\log S(t)) / d(t)$. Para avaliar o efeito de um conjunto de $m$ covariáveis $\boldsymbol{x}^{\prime}=\left(x_{1}, \ldots, x_{m}\right)$ em T, David (1972) propõe utilizar uma função de risco proporcional dada por

$$
h(t, \boldsymbol{x})=h_{0}(t) \exp \left(\boldsymbol{x}^{\prime} \boldsymbol{\beta}\right), \quad t \geq 0,
$$

em que $h_{0}(t)$ é uma função não-negativa arbitrária e $\boldsymbol{\beta}^{\prime}=\left(\beta_{1}, \ldots, \beta_{m}\right)$ um vetor de parâmetros desconhecidos, a ser estimado.

A análise de um modelo de regressão pode ser dividida em três estágios: especificação, ajuste do modelo e predição (Heller e Simonoff, 1992). Para o modelo semi-paramétrico de Cox, a especificação envolve a adequação da suposição de riscos proporcionais dada por (3.1), o ajuste envolve a estimação dos parâmetros $\boldsymbol{\beta}$ e $h_{0}(t)$. Entretanto, a terceira etapa da análise, a predição, não é feita pela equação de regressão ajustada, como em outros modelos. Como a estimação dos coeficientes de regressão $\boldsymbol{\beta}$ é feita com base no método de máxima verossimilhança parcial (EMVP), que dispensa a estimação da função de risco basal $h_{0}(t)$, a distribuição dos tempos de falha não é especificada e a predição é feita por meio da função de risco relativo dada por $\exp \left(\boldsymbol{x}^{\prime} \boldsymbol{\beta}\right)$.

O modelo semi-paramétrico de Cox é flexível o suficiente para comportar um número infinito de grupos, ou seja, manter as variáveis contínuas, mas a interpretação dos resultados exige considerar um número finito de grupos para avaliação dos riscos relativos. À vista disso, é frequente a categorização de variáveis contínuas à priori da especificação do modelo. Dessa forma, todos os indivíduos de um mesmo grupo são considerados como tendo o mesmo risco de falha. A forma como uma variável é agrupada, dependendo da quantidade de grupos e dos pontos de corte que os definem, pode interferir na significância dela para a predição do modelo. Então, apesar do modelo de riscos proporcionais ser um ótimo método para escolha 
de fatores prognósticos que influenciam a sobrevivência, ele pode ser comprometido pela especificação das categorias da covariável inicialmente contínua.

\subsection{Problemas oriundos da categorização de variáveis contínuas}

A simplicidade alcançada pelo agrupamento dos valores de uma variável contínua em grupos com características mais homogêneas conduz a vários problemas estatísticos que podem empobrecer a análise; ainda assim, o uso dessa abordagem permanece sendo a principal forma de apresentação dos dados e de avaliação da relação entre uma variável contínua e a reposta (Royston et al. (2006) e Bennette e Vickers (2012)). Mesmo em casos que a variável explicativa não estabelece relação linear com a resposta, em vez de recorrer a sua categorização, Weinberg (1995) pontuou que métodos alternativos para o uso integral da informação transmitida pela variável devem ser preferidos, como diferentes tipos de splines e polinômios fracionários (Royston e Altman, 1994).

Um dos principais problemas da categorização é a inevitável perda de informação. A maneira como os dados são coletados geralmente possui um erro de medida gerado por fatores comuns como a variação de laboratórios e a imprecisão dos meios de coleta; quando a natureza da variável é alterada, o erro de medida aumenta. A informação que a variável contínua fornece pode ser alterada pela escolha dos pontos de corte, pois indivíduos que possuam valores muito próximos, mas que estejam em lados opostos ao ponto de corte, deverão ser tratados como se tivessem características muito diferentes ao invés de parecidas (Royston et al., 2006).

Um argumento utilizado erroneamente é afirmar que valores coletados de forma imprecisa, quando agrupados, se tornam mais precisos e confiáveis. Na verdade, a categorização de uma variável contínua reduz a correlação com os verdadeiros valores populacionais da variável (desconhecidos) e, geralmente, reduz a precisão de medida, subestima a força da relação entre as variáveis e prejudica o poder de detectar a verdadeira relação com a resposta (Maxwell e Delaney (1993) e Taylor e Yu (2002)). O fato dos resultados permanecerem estatisticamente significativos é outro argumento inválido para defender o uso da categorização nos casos em que mais de uma variável preditora é categorizada ou testes múltiplos são aplicados para seleção dos pontos de corte, pois a significância estatística alcançada pode ser resultado da inflação da taxa do erro tipo I (Maxwell e Delaney, 1993).

No caso em que a variável é dicotomizada, a amostra passa a ser representada por apenas dois grupos e a chance de ocorrer a junção de indivíduos com riscos muito distintos em um mesmo grupo é alta, sendo este fenômeno conhecido por pooling data. Suponha que a relação entre a variável e a reposta seja descrita por uma curva em forma de "U", certamente a dicotomia não será capaz de identificar que os extremos se comportam de uma maneira e a parte central de outra; apenas um ponto de corte irá separar grupos em que ambos terão valores altos e baixos, impedindo a identificação de um padrão para cada categoria, sendo preferível a politomia. Quando o verdadeiro risco cresce (ou decresce) monotonicamente 
com o nível da variável de interesse, a variabilidade aparente do risco será aumentada de acordo com o número de grupos, podendo ser seriamente subestimada no caso de dicotomia (Altman, 2005).

Segundo (Cohen, 1983), forçar uma variável contínua a ser representada por apenas dois valores é equivalente a perder de um terço a dois terços dos dados, levando a uma séria perda de poder de detecção da relação entre a variável e a resposta; mesmo ao aumentar o número de categorias, a perda de poder ainda pode acontecer. Descartar um terço dos dados é bastante penoso em pesquisas com poucas observações, e aumentar o tamanho da amostra pode não ser viável por motivos financeiros ou por falta de observações disponíveis.

Alguns autores quantificam a perda associada ao uso da categorização fazendo uso da eficiência relativa assintótica (ARE, do inglês, Asymptotic Relative Efficiency) definida no Apêndice B por (B.1). Os valores de ARE pertencem ao intervalo $[0, \infty)$, em que ARE $>1$ indica que o modelo categórico apresenta maior eficiência do que o modelo contínuo. Segundo Lagakos (1988), se a variável $X$ seguir distribuição Normal e for dicotomizada pela mediana, a eficiência relativa aos dados não agrupados é de 65\%. Para o caso em que a variável é exponencialmente distribuída, a eficiência relativa aos dados não agrupados é de apenas $48 \%$.

Muitos autores apresentam argumentos consistentes a fim de provar que a categorização em três ou mais grupos gera menor perda de informação do que a dicotomia, facilitando a absorção da informação fornecida pela variável contínua e identificando melhor a sua forma funcional. Mas apesar das vantagens, aumentar a quantidade de grupos pode superestimar a variabilidade dos riscos entre as categorias e aumentar a chance de formação de grupos com poucas observações. Portanto, a escolha do número de grupos é uma questão tão importante quanto a escolha dos valores que os dividirão (Altman, 2005) e deve levar em conta o tamanho da amostra e a opinião do pesquisador da área. Além disso, para dados de sobrevivência, é importante evitar a formação de grupos em que falhas não sejam observadas (Clark et al. (2003) e Morgan e Elashoff (1986)).

Connor (1972) avaliou a perda de eficiência ao agrupar os valores de variáveis contínuas em $k$ grupos baseando-se no método da eficiência relativa assintótica dada por (B.1). Os pontos de corte "ótimos" foram selecionados ao maximizar a eficiência assintótica dos testes. Para os casos em que a variável a ser categorizada segue distribuição Normal, Uniforme e Exponencial, a eficiência relativa aos dados não agrupados, em porcentagem, para $k=$ 2,3,4,5,6 é apresentada na Tabela 3.1. Note que os valores da ARE são crescentes em relação a $k$.

No caso de politomia, existem diversas estratégias de análise permitindo diferentes maneiras de codificação, o que prejudica a comparação com outros estudos. Supondo a existência de $k$ categorias, é usual criar $k$-1 variáveis indicadoras binárias (ou variáveis dummies). A fim de testar o efeito da variável categorizada, todas as $k-1$ dummies podem ser avaliadas de uma única vez por um único teste com $k$-1 graus de liberdade. Mas essa abordagem perde em poder para o teste na presença da variável ainda contínua. 
Tabela 3.1: Eficiência assintótica para a variável agrupada em $k$ grupos relativa aos dados não agrupados, em porcentagem (\%).

\begin{tabular}{lccccc}
\hline \multirow{2}{*}{ Distribuição } & \multicolumn{5}{c}{$\boldsymbol{k}$} \\
\cline { 2 - 6 } & 2 & 3 & 4 & 5 & 6 \\
\hline Normal & 65 & 81 & 88 & 92 & 94 \\
Uniforme & 75 & 89 & 94 & 96 & 97 \\
Exponencial & 65 & 82 & 89 & 93 & 95 \\
\hline
\end{tabular}

Maxwell e Delaney (1993) e Taylor e Yu (2002) discutem mais a respeito do viés das estimativas, perda de eficiência e significância estatística espúria decorrentes da dicotomia e categorização de variáveis explicativas contínuas. Quando o modelo possui observações que fogem das suposições usuais de uma modelagem estatística (por exemplo, variáveis não independentes e que não seguem a mesma distribuição de probabilidade) ou, ainda, quando mais de uma variável é categorizada, todos os problemas acima são agravados (Mazumdar e Glassman, 2000).

\subsection{Métodos descritivos de categorização}

No processo de categorização de variáveis, os pontos de corte que limitam os grupos deveriam, idealmente, ser propostos pelo pesquisador da área ou pela literatura do assunto, mas nem sempre isso é possível. Nestes casos, um estudo empírico baseado em amostras da população de interesse pode ser realizado a fim de auxiliar no processo da categorização. Uma vez colhida a amostra e ajustado o modelo que relaciona a variável a ser categorizada $X$, com a variável dependente $Y$, a análise gráfica desta relação pode auxiliar no processo de busca dos pontos de corte mais adequados. Se a curva que descreve a relação entre X e Y sofrer alterações no comportamento gráfico, então a busca pelo valor de $X$ que melhor divide a amostra em grupos com respostas distintas deve ser feita nesse intervalo de pontos que apresenta tal alteração.

Além de indicar intervalos de busca, a análise gráfica pode auxiliar na identificação da quantidade ideal de grupos em que a amostra deve ser dividida segundo a variável $X$. A Figura 3.1 indica três situações hipotéticas de possíveis relações entre variável explicativa e resposta. Se a relação das variáveis for da forma dos Gráficos A ou do B, monotonicamente crescente (ou decrescente), como a relação entre o tamanho do tumor e risco de morte, a dicotomia pode ser aplicada. Inclusive, a curva A apresenta a situação ideal em que o ponto de corte é revelado a partir da análise gráfica. O Gráfico C indica uma possível relação em que um único ponto de corte não é capaz de dividir as observações em grupos de diferentes riscos como, por exemplo, a relação entre a variável pressão arterial e risco de morte, em que valores baixos e altos estão associados ao aumento do risco de óbito (Mazumdar e Glassman, 2000).

As relações que se apresentam na forma da linha do Gráfico B não sugerem indícios de mudança no comportamento da variável e, então, a categorização não é indicada. Nos casos 
em que a categorização é requerida, é possível selecionar bons pontos de corte ao associar a análise gráfica à busca sistemática, avaliando os possíveis pontos segundo critérios estatísticos (Williams et al., 2006).

A

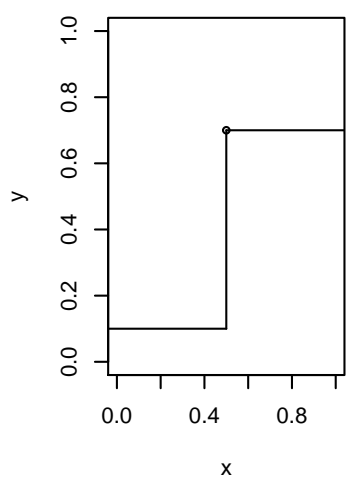

B

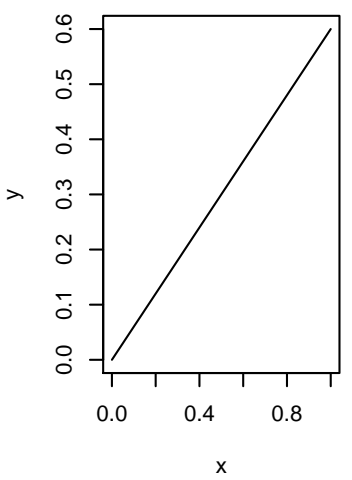

C

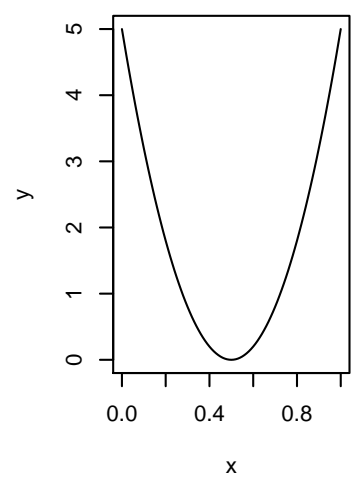

Figura 3.1: A variável resposta genérica é representada por uma variável contínua. Gráfico A: representa uma função step ou degrau e indica a situação ideal em que o gráfico é capaz de revelar o ponto de corte adequado para a variável, raramente a relação funcional entre as variáveis resposta e explicativa é dessa forma. Gráfico B: indica uma relação monótona crescente entre as variáveis $x$ e y; os pontos de corte para divisão dos grupos de risco não são aparentes. Gráfico C: apresenta uma possivel relação não monótona entre as variáveis e sugere a politomia da variável $x$.

\section{Gráficos}

Mazumdar e Glassman (2000) propõe gráficos distintos de acordo com a natureza das variáveis envolvidas para busca dos potenciais pontos de corte. Para descrição dos gráficos a seguir, suponha $X$ uma variável independente contínua, $Z^{*}=\left(Z_{1}, \ldots, Z_{p-1}\right)^{\prime}$ um vetor de covariáveis de dimensão $p-1, Y$ a variável dependente e $\boldsymbol{Z}=\left(X, Z_{1}, \ldots, Z_{p-1}\right)^{\prime}$ o vetor de covariáveis $p$-dimensional.

Resposta sem censura: Se $Y$ é uma variável categórica, o gráfico de dispersão sobre os valores da variável $X$ idealmente apresenta o grau da separação dos indivíduos nos diferentes grupos de risco (Gráfico A da Figura 3.2). Se o gráfico for da forma de uma função degrau (Figura 3.1, Gráfico A), então os pontos de corte são explicitados. Usualmente o gráfico não se apresenta dessa última forma. Para o caso em que $Y$ é uma variável dicotômica e $X$ tem valores muito dispersos, um gráfico para dados agrupados é uma boa opção; os valores de $X$ são agrupados em decis ou outro quantil mais adequado à amostra e, então, a média da covariável dentro de cada decil é plotada contra as médias de $Y$ para aquele decil; por ser binária, a média de $Y$ equivale à proporção de $Y=1$ em cada decil. Um método utilizado para ajudar a revelar a relação implícita entre as variáveis contínuas $X$ e $Y$ é a curva suavizada pelo algoritmo LOWESS (Locally wheighted regression smoothed scatter) sobre o gráfico de dispersão, baseado em sucessivos ajustes de retas de mínimos quadrados ponderados (Gráfico B da Figura 3.2). Quebras bruscas na curva podem fornecer um indício 
para seleção dos valores que separam grupos de riscos distintos.

A

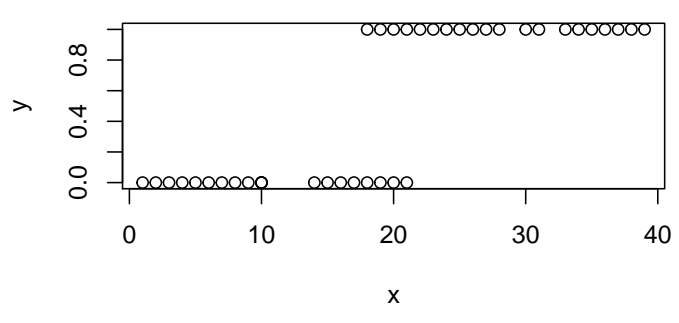

B

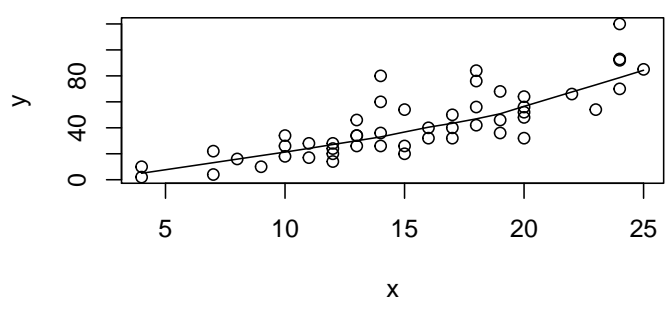

Figura 3.2: Gráfico A: representa o gráfico de dispersão para variável $Y$ binária e $X$ contínua; a curva indica uma leve alteração no comportamento da variável $x$ nos valores próximos a $x=20$. Gráfico B: representa o gráfico de dispersão para variáveis $X$ e $Y$ continuas com a curva suavizada via método LOWESS; a curva não indica mudança aparente no comportamento de $X$.

Resposta censurada: Quando $Y$ é uma variável resposta que apresenta censura, como no caso de tempos de sobrevivência, os gráficos acima podem não ser informativos devido à falta de acompanhamento da informação completa a respeito de $Y$. Para dados de sobrevivência é bastante usual a utilização dos gráficos baseados nas estimativas dos tempos de sobrevivência e nos resíduos martingal.

\section{Resíduo Martingal:}

Os resíduos martingal são vistos como uma estimativa do número de falhas em excesso observado nos dados mas não predito pelo modelo e são comumente utilizados para verificação da adequabilidade do modelo e da melhor forma funcional para cada covariável do modelo ajustado (logarítmica, quadrática, linear, categórica etc.).

Considere que $Y$ representa o tempo de falha de um indivíduo e $C$, uma variável aleatória independente de $Y$, representa o tempo de censura associado a esse indivíduo. Suponha uma amostra de tamanho $n$, em que a $i$-ésima observação é representada por $\left(y_{i}, \delta_{i}, \boldsymbol{z}_{i}\right)$, para $i=1,2, \ldots, n, \operatorname{com} y_{i}=\min \left(Y_{i}, C_{i}\right), \delta_{i}=I\left(Y_{i} \leq C_{i}\right)$ e $\boldsymbol{z}_{i}=\left(z_{1 i}, \ldots, z_{(p-1) i}, x_{i}\right)^{\prime}$ os valores das covariáveis medidas no $i$-ésimo indivíduo.

Seja $\boldsymbol{\beta}=\left(\beta_{1}, \ldots, \beta_{p-1}, \gamma\right)^{\prime}$ o vetor de parâmetros de regressão associados ao vetor de covariáveis $\boldsymbol{Z}$ de dimensão $p$, em que $\gamma$ é o parâmetro associado a variável a ser categorizada $X$. Para dados censurados à direita e variáveis não dependentes do tempo, os resíduos 
martingal para o modelo de Cox são definidos por:

$$
\hat{m}_{i}=\delta_{i}-\hat{\Lambda}_{0}\left(y_{i}\right) \exp \left\{x_{i} \hat{\gamma}+\sum_{k=1}^{p-1} z_{i k} \hat{\beta}_{k}\right\}=\delta_{i}-\hat{e}_{i}
$$

em que $\hat{e}_{i}$ são os resíduos de Cox-Snell, $i=1,2, \ldots, n, \hat{\boldsymbol{\beta}}$ é o vetor de parâmetros estimados obtidos ao maximizar a verossimilhança parcial e $\hat{\Lambda}_{0}(\cdot)$ é a função de taxa de falha acumulada estimada pelo estimador de Breslow (Breslow, 1972) dado por:

$$
\hat{\Lambda}_{0}(y)=\sum_{j: y_{j}<y} \frac{d_{j}}{\sum_{l \in R_{j}} \exp \left\{\boldsymbol{z}_{l} \hat{\boldsymbol{\beta}}\right\}},
$$

em que $d_{j}$ é o número de falhas no instante de tempo $y_{j}$ e $R_{j}$ é o conjunto de índices de indivíduos em risco no instante $y_{j}^{-}$(Colosimo e Giolo, 2006).

A curva suavizada pelo método LOWESS do gráfico de dispersão feito com base nos pares $\left(x_{i}, \hat{m}_{i}\right)$, para $i=1,2, \ldots, n$, deve indicar a melhor forma funcional da variável $X$. Se a curva apresentar uma mudança grosseira em um determinado valor de $X$, então uma versão categórica da variável pode ser indicada. Outros comportamentos da curva podem sugerir outros tipos de transformações na variável (Colosimo e Giolo, 2006).

\section{Tempo de falha predito por Kaplan-Meier:}

Suponha $Y$ definido como no gráfico dos resíduos martingal. Uma forma de analisar graficamente os tempos de falha preditos pelo método de Kaplan-Meier, além das usuais curvas de Kaplan-Meier (Colosimo e Giolo, 2006), agrupa os valores de $X$ de modo a manter uma proporção razoável de falhas observadas dentro de cada grupo. E então, os tempos de sobrevivência medianos, ou seja, os valores de $y$ para os quais $\hat{S}(y)=0.5$ dentro de cada grupo de $X$ são estimados. O gráfico do valor médio de $X$ em cada grupo versus os tempos preditos pode auxiliar na investigação sobre a relação funcional entre as variáveis explicativa e resposta. Traçar a linha que une os pontos do gráfico pode auxiliar na visualização da relação entre $X$ e $Y$.

Tempo de falha predito pelo modelo de Cox:

Suponha as variáveis e os parâmetros definidos como no gráfico dos resíduos Martingal. A função de sobrevivência no modelo de Cox também pode ser definida como

$$
\hat{S}(y \mid \boldsymbol{z})=\left[S_{0}(y)\right]^{\exp \left\{\boldsymbol{z}^{\prime} \hat{\boldsymbol{\beta}}\right\}}
$$

em que $y$ é o tempo de sobrevivência observado, $\hat{\boldsymbol{\beta}}$ é o coeficiente de regressão de Cox obtido pela maximização da verossimilhança parcial e $\hat{S}_{0}(y)$ é uma estimativa suavizada da função de sobrevivência latente (Heller e Simonoff, 1992). A função de sobrevivência estimada, $\hat{S}(y \mid \boldsymbol{z})$, representa a proporção $q$ de indivíduos que permanece livre de falha. 
De modo geral, o q-ésimo percentil da distribuição dos tempos de sobrevivência é predito ao encontrar o instante de tempo $\hat{y}$ que satisfaz

$$
\hat{S}_{0}(\hat{y})=[q]^{\exp \left\{-\boldsymbol{z}^{\prime} \hat{\boldsymbol{\beta}}\right\}}
$$

para cada valor observado da variável a ser categorizada, $x$. O gráfico obtido ao plotar $\hat{y} \times x$ pode indicar se a suposição de monotonicidade na relação entre as variáveis é válida, e auxiliar na seleção de um ou mais pontos de corte ou definir um intervalo de busca.

É usual definir $q=0.5$ para estimar os tempos de sobrevivência. Dessa forma, o tempo de falha mediano predito para um dado $x$ é o valor que satisfaz

$$
\hat{S}_{0}(\hat{y})=[0.5]^{\exp \left\{-\boldsymbol{z}^{\prime} \hat{\boldsymbol{\beta}}\right\}}
$$

\subsection{Métodos de seleção de ponto de corte}

Os métodos mais fáceis de serem aplicados para seleção de pontos de corte são orientados pelos dados, ou seja, aqueles que baseiam-se na distribuição da covariável a ser categorizada. Os métodos mais comuns listados na literatura são: $(i)$ usar os quantis como limitadores dos grupos; (ii) no caso de dados de sobrevivência, dividir em categorias com a mesma proporção ou mesmo número de eventos observados em cada uma delas; (iii) dividir os indivíduos em grupos de mesma largura, ou seja, escolher números inteiros (usualmente múltiplos de 5 ou 10) como pontos de corte da variável e (iv) usar o limite superior de um intervalo de referência como ponto de corte. Uma última opção é usar os pontos de corte definidos em estudos anteriores, caso disponíveis.

O uso de quantis é o método mais usado para categorização, a edição do The American Journal of Epidemiology de outubro de 2009 apontou que a cada seis artigos publicados em seu jornal, quatro recorreram a alguma forma de categorização baseada em quantis e apenas dois mantiveram as variáveis contínuas (Bennette e Vickers, 2012). Esta última é intuitivamente uma boa medida de separação (Clark et al., 2003), entretanto, é uma abordagem altamente relacionada aos dados e, portanto, as conclusões não são facilmente comparáveis com estudos baseados em amostras diferentes que, provavelmente, fornecem pontos de corte distintos. Além disso, quantis definidos por valores mais altos da variável tornam as médias de cada grupo mais altas (Altman e Royston, 2006). Os custos estatísticos causados pela escolha da mediana amostral para dicotomia de uma variável explicativa contínua são discutidos em Lausen e Schumacher (1996), Faraggi e Simon (1996), Mazumdar et al. (2003), Maxwell e Delaney (1993), Rota et al. (2015), Tueller et al. (2016) e outros.

O método (ii) também retorna valores de ponto de corte dependentes da amostra, o que dificulta a extensão dos resultados a outros conjuntos de dados, enquanto que o método (iii) apresenta como grande vantagem a comparação com estudos baseados em diferentes amostras e, também, é esteticamente agradável. É pouco usual a aplicação do método (vi) para categorização de variáveis. 
A seguir, serão apresentados métodos de seleção de pontos de corte orientados pela resposta. Tais métodos sugerem testar possíveis valores de ponto de corte e selecionar aquele (ou aqueles, no caso de politomia) que retorna o melhor resultado segundo algum critério estatístico. Apesar dessa abordagem ser considerada mais adequada do que a abordagem orientada pelos dados (Klein e Wu, 2004), problemas inerentes à categorização permanecem, além do acréscimo do problema do aumento da taxa do erro tipo I provocado pelos testes múltiplos (Altman e Royston, 2006).

\subsubsection{Método do valor- $p$ mínimo}

O método mais comum de seleção dos pontos de corte "ótimos" é chamado método do valor-p mínimo (MPMIN) ou, equivalentemente, método da estatística máximal, pois utiliza como critério de seleção o ponto (ou vetor de pontos) de corte que maximiza uma determinada estatística de teste e, consequentemente, minimiza o valor- $p$ associado. O algoritmo para busca e seleção dos pontos de corte via MPMIN pode ser encontrado em diversos artigos como em Royston et al. (2006), Hilsenbeck e Clark (1996) e Buettner et al. (1997), por exemplo. Usualmente, os artigos referentes à esta técnica indicam sua aplicação em modelos univariados e no caso em que deseja-se comparar dois grupos definidos pelo ponto de corte a ser estimado; entretanto, há discussões sobre os casos em que a variável a ser categorizada precisa ser dividida em três ou mais grupos e sobre o caso em que ela está inserida originalmente em um modelo multivariado (Mazumdar e Glassman (2000) e Mazumdar et al. (2003)).

A politomia é mais indicada para os casos em que a variável explicativa não possua relação linear ou monótona com a resposta. Variáveis como a pressão arterial e o IMC são exemplos de variáveis que não seriam bem representadas por uma variável binária e são consideradas fatores prognósticos importantes de muitas doenças. Apesar da facilidade em encontrar exemplos de variáveis que seriam melhor representadas por três ou mais grupos, pouco é discutido sobre métodos de politomia. Além da questão da quantidade de grupos, acredita-se que os pontos de corte de variáveis contínuas inseridas em um modelo multivariado deveriam ser estimados no cenário multivariado, uma vez que a presença de outras variáveis explicam parte da variabilidade da variável dependente e poderiam auxiliar na estimativa de pontos de corte mais adequados. Usualmente, a busca pelo ponto de corte ideal é feita a partir de um modelo univariado que só contém a variável a ser categorizada, e então, a variável categorizada é inserida no modelo multivariado. Devido à importância desses últimos casos, a extensão do MPMIN para a politomia e para o cenário multivariado também são apresentados (Schumacher et al. (1997), Taylor e Yu (2002) e Mazumdar et al. (2003)).

Para busca dos pontos de corte "ótimos", independentemente do critério de seleção ser o valor- $p$ mínimo, o primeiro passo é a definição dos valores da variável contínua $X$ que devem ser testados como potenciais pontos de corte. De modo a garantir uma quantidade 
razoável de observações (e de falhas) em cada categoria, deve-se restringir a busca entre os $80 \%$ ou $90 \%$ valores centrais da amostra, sendo que outras restrições podem ser sugeridas pela análise gráfica ou por indicação dos pesquisadores da área. Definido o intervalo $\mathcal{R}$ de potenciais pontos de corte, supondo que deseja-se dividir os valores de $X$ em $r$ grupos, $r$ fixado à priori daa aplicação do método, vetores $\boldsymbol{c}$ de dimensão $(r-1)$ são definidos a partir dos valores contidos em $\mathcal{R}$. Agora, considerando o MPMIN, o segundo passo é realizar um teste estatístico que verifique a influência da variável categorizada por $\boldsymbol{c}$ sobre a variável dependente, para cada possível valor de $\boldsymbol{c}$. Opta-se pelo vetor que retornar o menor valor- $p$ associado ao teste.

\section{Cenário univariado e testes não-paramétricos}

Uma vantagem de realizar as buscas pelo ponto de corte no cenário univariado é que as expressões dos testes estatísticos que verificam a força de associação entre a variável categorizada e resposta tomam suas formas mais simples comparado ao cenário multivariado. Nesse contexto, os testes Score e logrank são os mais citados na literatura para aplicação do MPMIN (Faraggi e Simon (1996), Schumacher et al. (1997), Mazumdar e Glassman (2000) e Mazumdar et al. (2003)). Além disso, vale lembrar que, para dicotomia, os testes Score e logrank são equivalentes.

\section{Dicotomia}

No caso de dicotomia, o teste logrank avalia a igualdade das duas curvas de sobrevivência, $S_{1}(t)$ e $S_{2}(t)$, referentes aos dois grupos gerados pelo ponto de corte fixado $c$, em que $S(t)=$ $P(T>t)$ e $T$ é a variável que representa o tempo até a ocorrência de um evento de interesse ou o tempo que a observação foi censurada. Suponha $k$ tempos de falha observados, distintos e ordenados avaliados sob a amostra completa $t_{1}<\ldots<t_{k}$. Considere que $d_{j}$ falhas ocorrem no instante $t_{j}$ e $n_{j}$ é o número de indivíduos em risco no instante de tempo imediatamente inferior a $t_{j}$ na amostra completa, para $j=1,2, \ldots, k$. O número de falhas e o de indivíduos em risco para cada grupo $i(i=1,2)$ são representados por $d_{i j}$ e $n_{i j}$.

Para cada tempo de falha $t_{j}$ e ponto de corte $c$ fixado, os dados podem ser dispostos em uma tabela de contingência $2 \times 2$, como representada pela Tabela 3.2, em que $n_{i j^{-}} d_{i j}$ indica o número de sobreviventes não censurados na categoria $i$ (Mazumdar e Glassman, 2000).

Tabela 3.2: Tabela de contingência $2 \times 2$ usada para o teste logrank no instante $t_{j}$ e ponto de corte c fixado.

\begin{tabular}{c|cc|c} 
& $X \leq c$ & $X>c$ & Totais \\
\hline Falha & $d_{1 j}$ & $d_{2 j}$ & $d_{j}$ \\
Não falha & $n_{1 j}-d_{1 j}$ & $n_{2 j}-d_{2 j}$ & $n_{j}-d_{j}$ \\
\hline Totais & $n_{1 j}$ & $n_{2 j}$ & $n_{j}$
\end{tabular}

Condicional à experiência de falha e censura até o instante $t_{j}$ e ao número de falhas no tempo $t_{j}$ (fixando as marginais), a distribuição de $d_{2 j}$ é hipergeométrica com média 
$w_{2 j}=n_{2 j} d_{j} n_{j}^{-1}$ e variância $V_{2 j}=n_{2 j}\left(n_{j}-n_{2 j}\right) d_{j}\left(n_{j}-d_{j}\right) n_{j}^{-2}\left(n_{j}-1\right)^{-1}$. Defina

$$
L=\frac{\left[\sum_{j=1}^{k}\left(d_{2 j}-w_{2 j}\right)\right]^{2}}{\sum_{j=1}^{k} V_{j 2}} .
$$

Isto posto, a estatística $d_{2 j}-w_{2 j}$ tem média zero e variância $V_{2 j}$. Supondo que as $k$ tabelas de contingência são independentes, a estatística representada por (3.3) é um teste para a igualdade das funções de sobrevivência que, sob $H_{0}: S_{1}(t)=S_{2}(t)$ para todo $t$ no período de acompanhamento, tem uma distribuição assintótica qui-quadrado com um grau de liberdade. A estatística de teste é calculada para cada potencial ponto de corte e aquele que resultar no maior valor da estatística será escolhido como o ponto de corte "ótimo".

\section{Politomia}

No caso de politomia, para testar a igualdade de $r>2$ funções de sobrevivência $S_{1}(t), \ldots$, $S_{r}(t)$, definidas por um vetor $c$ de pontos de corte, em que $c$ é de dimensão $r$ - 1 , basta usar a versão generalizada do teste logrank. Considere que a notação permanece a mesma que para o caso anterior, mas com o índice $i$ variando entre 1 e $r$. Dessa forma, para cada instante $t_{j}$, $j=1,2, . ., k$, os dados podem ser agrupados em tabelas de contingência $2 \times r$ com $d_{i j}$ falhas e $n_{i j}-d_{i j}$ sobreviventes na coluna $i$. Por exemplo, no caso de tricotomia $(r=3)$, para dois pontos de corte $c_{1}$ e $c_{2}$ fixados, a tabela é da forma:

Tabela 3.3: Tabela de contingência $2 \times 3$ exemplificando a versão generalizada do teste logrank para $r=3$ no instante $t_{j}$ e pontos de corte $c_{1}$ e $c_{2}$ fixados.

\begin{tabular}{c|ccc|c} 
& $X \leq c_{1}$ & $c_{1}<X \leq c_{2}$ & $X>c_{2}$ & Totais \\
\hline Falha & $d_{1 j}$ & $d_{2 j}$ & $d_{3 j}$ & $d_{j}$ \\
Não falha & $n_{1 j}-d_{1 j}$ & $n_{2 j}-d_{2 j}$ & $n_{3 j}-d_{3 j}$ & $n_{j}-d_{j}$ \\
\hline Totais & $n_{1 j}$ & $n_{2 j}$ & $n_{3 j}$ & $n_{j}$
\end{tabular}

Condicional à experiência de falha e censura até o instante $t_{j}$ e ao número de falhas no tempo $t_{j}$ (fixando as marginais), a distribuição conjunta de $d_{2 j}, \ldots, d_{r j}$ é hipergeométrica multivariada, em que $d_{i j}$ tem média $w_{i j}=n_{i j} d_{j} n_{j}^{-1}$, variância $\left(V_{j}\right)_{i i}=n_{i j}\left(n_{j}-n_{i j}\right) d_{j}\left(n_{j}-\right.$ $\left.d_{j}\right) n_{j}^{-2}\left(n_{j}-1\right)^{-1}$ e a covariância de $d_{i j}$ e $d_{l j}$ é dada por $\left(V_{j}\right)_{i l}=-n_{i j} n_{l j} d_{j}\left(n_{j}-d_{j}\right) n_{j}^{-2}\left(n_{j}-1\right)^{-1}$.

Sendo assim, a estatística $\boldsymbol{v}_{j}^{\prime}=\left(d_{2 j}-w_{2 j}, \ldots, d_{r j}-w_{r j}\right)$ possui média zero e matriz covariância $\boldsymbol{V}_{j}$ de dimensão $r$-1, em que $\left(V_{j}\right)_{i i}$ ocupa a diagonal principal, $i=1, . ., r$ e fora dela os elementos $\left(V_{j}\right)_{i l}$, para $i, l=1, \ldots, r$. Somando $\boldsymbol{v}_{j}$ para todos os instantes distintos de falha, obtém-se um vetor $\boldsymbol{v}$ de dimensão $(r-1)$ que contém as diferenças entre os totais observados e esperados de falha

$$
\boldsymbol{v}=\sum_{j}^{k} \boldsymbol{v}_{j}
$$

Para $k$ tabelas de contingência independentes, a variância da estatística $\boldsymbol{v}$ é dada por $\boldsymbol{V}=\boldsymbol{V}_{1}+\ldots+\boldsymbol{V}_{k}$ e um teste aproximado para igualdade das $r$ funções de sobrevivência 
pode ser baseado na estatística

$$
L=\boldsymbol{v}^{\prime} \boldsymbol{V}^{-1} \boldsymbol{v}
$$

que, sob hipótese nula, tem uma distribuição aproximada qui-quadrado com $r-1$ graus de liberdade. Para mais detalhes sobre o teste logrank verificar Colosimo e Giolo (2006).

Para cada possível vetor de pontos de corte $\boldsymbol{c}^{\prime}=\left(c_{1}, \ldots, c_{r-1}\right)$ o teste é realizado e seleciona-se o vetor associado ao menor valor- $p$ como o conjunto de pontos de corte que melhor diferenciam as curvas de sobrevivência.

Como a estatística qui-quadrado baseada neste teste é muito sensível ao tamanho da amostra, Mazumdar e Glassman (2000) sugerem uma avaliação conjunta do menor valor- $p$ e dos riscos relativos. A ideia é selecionar o ponto ou o vetor $c$ que leva a grupos com maior diferença entre os riscos.

\section{Cenários univariado e multivariado baseados em modelo de regressão}

Outros possíveis testes indicados para estimação dos pontos de corte, mas que permitem a inserção de outras covariáveis significativas na análise, são os testes de razão de verossimilhanças (TRV), Wald e Score (Klein e Wu (2004) e Holländer et al. (2004)). A dedução dos três testes citados faz uso de resultados assintóticos e métodos para amostras grandes; tanto a teoria assintótica quanto a dedução dos testes estão apresentados no Apêndice A.

No cenário univariado, suponha que deseja-se categorizar a variável contínua $X$ em $r$ grupos. Então, para cada possível vetor de pontos de corte $\boldsymbol{c}^{\prime}=\left(c_{1}, \ldots, c_{r-1}\right)$, os três testes são aplicados a fim de testar $H_{0}: \boldsymbol{\beta}=\mathbf{0}$, em que $\boldsymbol{\beta}$ é o vetor de parâmetros de regressão associado às $r$ categorias da variável categorizada a partir de $X$. Para cada um dos três testes, o vetor que resultar no menor valor- $p$ é escolhido como vetor "ótimo".

Estatisticas avaliadas em um subconjunto dos parâmetros

Se o modelo no qual a variável $X$ está inserida for multivariado, para avaliação da relevância desta variável no modelo é necessário restringir o teste de hipóteses somente aos parâmetros associados a ela. Testes e estimativas para um subconjunto de parâmetros podem ser obtidos particionando devidamente o vetor $\boldsymbol{\theta}$ e as estatísticas associadas $\boldsymbol{U}(\boldsymbol{\theta}), \boldsymbol{I}\left(\boldsymbol{\theta}_{0}\right)$ e $\boldsymbol{I}^{-1}\left(\boldsymbol{\theta}_{0}\right)$ (Lawless, 2011). Para os testes abaixo, suponha que deseja-se dividir a variável contínua $X$ em $k$ grupos e que $X^{\prime}$ representa a variável categorizada a partir de $X$.

Seja $\boldsymbol{\theta}^{\prime}=\left(\boldsymbol{\theta}_{1}^{\prime}, \boldsymbol{\theta}_{2}^{\prime}\right)$ o vetor de parâmetros particionado, em que $\boldsymbol{\theta}_{1}$ é o vetor $k$-dimensional associado às categorias de $X^{\prime}$ e $\boldsymbol{\theta}_{2}$ é o vetor $(p-k)$-dimensional associado ao restante das variáveis do modelo. As partições para as estatísticas citadas ficam da seguinte forma:

$$
\boldsymbol{U}(\boldsymbol{\theta})=\left(\begin{array}{c}
\boldsymbol{U}_{1}(\boldsymbol{\theta}) \\
\boldsymbol{U}_{2}(\boldsymbol{\theta})
\end{array}\right), \quad \boldsymbol{I}(\boldsymbol{\theta})=\left(\begin{array}{cc}
\boldsymbol{I}_{11}(\boldsymbol{\theta}) & \boldsymbol{I}_{12}(\boldsymbol{\theta}) \\
\boldsymbol{I}_{21}(\boldsymbol{\theta}) & \boldsymbol{I}_{22}(\boldsymbol{\theta})
\end{array}\right) \mathrm{e}
$$




$$
\boldsymbol{I}^{-1}(\boldsymbol{\theta})=\left(\begin{array}{cc}
\boldsymbol{I}^{11}(\boldsymbol{\theta}) & \boldsymbol{I}^{12}(\boldsymbol{\theta}) \\
\boldsymbol{I}^{21}(\boldsymbol{\theta}) & \boldsymbol{I}^{22}(\boldsymbol{\theta})
\end{array}\right) .
$$

De modo geral, para um dado valor de $\boldsymbol{\theta}_{1}=\boldsymbol{\theta}_{01}$, seja $\tilde{\boldsymbol{\theta}}_{2}\left(\boldsymbol{\theta}_{01}\right)$ o estimador de máxima verossimilhança (EMV) de $\boldsymbol{\theta}_{2}$ obtido por maximizar o logaritmo da verossimilhança parcial $l\left(\boldsymbol{\theta}_{01}, \boldsymbol{\theta}_{2}\right)=\log \left(L\left(\boldsymbol{\theta}_{01}, \boldsymbol{\theta}_{2}\right)\right)$ com repeito a $\boldsymbol{\theta}_{2}$ (Lawless, 2011). Esta última é denominada de verossimilhança perfilada e a função da log-verossimilhança perfilada é dada por

$$
\begin{aligned}
l_{k}\left(\boldsymbol{\theta}_{01}\right) & =\max _{\boldsymbol{\theta}_{2}} l\left(\boldsymbol{\theta}_{01}, \boldsymbol{\theta}_{2}\right) \\
& =l\left(\boldsymbol{\theta}_{01}, \tilde{\boldsymbol{\theta}}_{2}\left(\boldsymbol{\theta}_{01}\right)\right)
\end{aligned}
$$

Denote $\tilde{\boldsymbol{\theta}}=\left(\boldsymbol{\theta}_{01}, \tilde{\boldsymbol{\theta}}_{2}\left(\boldsymbol{\theta}_{01}\right)\right)$, então, sob $H_{0}: \boldsymbol{\theta}_{1}=\boldsymbol{\theta}_{01}$, as estatísticas Score, Wald e TRV são dadas, respectivamente, por:

$$
\begin{gathered}
S=\boldsymbol{U}_{1}^{\prime}(\tilde{\boldsymbol{\theta}})^{\prime} \boldsymbol{I}^{11}(\tilde{\boldsymbol{\theta}}) U_{1}(\tilde{\boldsymbol{\theta}}), \\
W=\left(\hat{\boldsymbol{\theta}}_{1}-\boldsymbol{\theta}_{01}\right)^{\prime} \boldsymbol{I}^{11}(\hat{\boldsymbol{\theta}})^{-1}\left(\hat{\boldsymbol{\theta}}_{1}-\boldsymbol{\theta}_{01}\right) \quad \mathrm{e} \\
T R V\left(\boldsymbol{\theta}_{01}\right)=2 l(\hat{\boldsymbol{\theta}})-2 l(\tilde{\boldsymbol{\theta}})=2 l_{k}\left(\hat{\boldsymbol{\theta}}_{1}\right)-2 l_{k}\left(\boldsymbol{\theta}_{01}\right) .
\end{gathered}
$$

Sob hipótese nula, todos os testes seguem distribuição assintótica $\chi^{2}$ com $k$ graus de liberdade, em que $k$ é a dimensão do vetor $\boldsymbol{\theta}_{01}$ e as matrizes $\boldsymbol{I}^{11}(\tilde{\boldsymbol{\theta}})$ e $\boldsymbol{I}^{11}(\hat{\boldsymbol{\theta}})$ são de dimensão $k \times k$.

O teste Score avaliado em um subconjunto de parâmetros perde a propriedade de não necessitar da estimação de parâmetros. Para aplicação do MPMIN, o modelo deve ser ajustado na presença da variável $X^{\prime}$ categorizada em $k$ grupos a partir da variável $X$ pelo vetor de pontos de corte $\boldsymbol{c}(k-1)$-dimensional. Nesse contexto, suponha que $H_{0}: \boldsymbol{\theta}_{1}=\mathbf{0}$, em que $\boldsymbol{\theta}_{1}$ é o parâmetro associada a variável $X^{\prime}$. Quando $X$ for dicotomizada e, consequentemente, $\theta_{1}$ for um escalar, para $\tilde{\boldsymbol{\theta}}=\left(0, \tilde{\boldsymbol{\theta}}_{2}(0)\right)$, as estatísticas acima tomam as suas formas mais simples, dadas por:

$$
\begin{gathered}
S=\boldsymbol{U}_{1}(\tilde{\boldsymbol{\theta}})^{2} i^{11}(\tilde{\boldsymbol{\theta}}), \\
W={\hat{\theta_{1}}}^{2} / \operatorname{Var}\left(\hat{\theta_{1}}\right) \quad e \\
T R V(0)=2 l_{k}\left(\hat{\boldsymbol{\theta}}_{1}\right)-2 l_{k}(0) .
\end{gathered}
$$

Para os testes acima $k=1$ e, portanto, as estatísticas seguem, sob $H_{0}$, distribuição assintótica qui-quadrado com 1 grau de liberdade. 


\section{Comparações múltiplas}

Ao aplicar métodos de seleção de pontos de corte que dependem de sucessivos testes estatísticos, chamados de testes múltiplos ou comparações múltiplas, como no caso do MPMIN, o grande problema da inflação da taxa do erro tipo I aparece. A fim de exemplificar o que acontece com as comparações múltiplas de modo geral, suponha que deseja-se estimar o vetor $\boldsymbol{c}$ que define a variável aleatória $X^{\prime}$ categorizada a partir da variável contínua $X$. Suponha que existam $b$ vetores de potenciais pontos de corte para $X$ e, portanto, para cada possível $\boldsymbol{c}$, uma estatística que testa $H_{0}: \boldsymbol{\theta}=\mathbf{0}$ é calculada, em que $\boldsymbol{\theta}$ é o vetor de parâmetros associado a $X^{\prime}$. Seja $\alpha$ o nível de significância pré-especificado de cada um dos testes, então, para $b$ testes múltiplos independentes, temos que:

$$
\begin{aligned}
P\left(\text { rejeitar } H_{0} \text { ao menos em um teste } \mid H_{0} \text { verdade }\right) & =1-P\left(\text { não rejeitar } H_{0} \mid H_{0} \text { verdade }\right) \\
& =1-(1-\alpha)^{b}
\end{aligned}
$$

Desse modo, a probabilidade de rejeitar a hipótese nula, mesmo que ela seja verdade, tende a 1 quando a quantidade de testes $b$ aumenta. Ou seja, quanto maior a quantidade de pontos ou vetores $c$ testados, maior é a probabilidade de um falso positivo. Note que, quanto maior a quantidade de grupos a serem formados a partir de $X$, a quantidade de testes também aumenta; assim sendo, a tricotomia tende a aumentar mais a taxa do erro tipo I do que a dicotomia de uma variável contínua. Para testes dependentes, como no caso do MPMIN que realiza testes de comparação dos grupos sobre a mesma amostra, o aumento na taxa do erro também ocorre (Buettner et al., 1997).

O efeito da seleção do ponto de corte "ótimo" sobre a probabilidade do erro tipo I foi avaliado por simulação em Faraggi e Simon (1996). Para cada amostra, 100 tempos de sobrevivência independentes e identicamente distribuídos (i.i.d.) foram gerados a partir de uma distribuição exponencial padrão, e para cada instante de tempo, um valor associado a uma covariável $X$ uniformemente distribuída com valores em [0,1], independente do tempo, foi gerado. O MPMIN foi aplicado para cada amostra e um histograma dos valores- $p$ mínimos retidos foi apresentado.

Assumindo $H_{0}$ verdadeira, ou seja, ausência de relação entre os tempos de sobrevivência e os valores da covariável, os valores- $p$ encontrados deveriam apresentar uma distribuição uniforme sobre o intervalo $[0,1]$. No entanto, o histograma apresentou uma distribuição assimétrica à direita e a probabilidade do valor- $p$ ser menor do que 0.05 se apresentou aproximadamente igual a $24 \%$.

Altman (2005) pontuou que a taxa global de falso positivo ao realizar os testes múltiplos fica em torno dos 40\% ao invés do usual 5\%. Consequentemente, haverá uma superestimação do verdadeiro impacto da variável sobre a resposta (Schumacher et al. (1997), Buettner et al. 
(1997) e Altman (2005)). Clark et al. (2003) sugerem não escolher pontos de corte baseados no MPMIN caso nenhum método de correção do viés seja aplicado, sendo preferíveis métodos orientados pelos dados, como a escolha de quantis, por exemplo. Portanto, métodos para correção do valor- $p$ e do possível viés na estimação dos pontos de corte são propostos mais para frente.

\subsubsection{Método da diferença das verossimilhanças}

Para o modelo de riscos proporcionais de Cox, é por meio da função de verossimilhança parcial dada por (A.1) ou (A.2) que usualmente faz-se as estimações para os parâmetros desconhecidos que medem os efeitos das covariáveis sobre a função de taxa de falha.

O método da diferença das verossimilhanças (MDV) compara as verossimilhanças dos modelos na presença da variável originalmente contínua e na presença da variável categorizada a partir desta variável contínua (Vinh-Hung et al., 2009). Devido aos problemas inerentes à categorização, conceitualmente, espera-se que a verossimilhança do modelo que incorpora uma variável categorizada, $\left[L_{c a t}\left(\boldsymbol{\beta}^{*}\right)\right]$, seja menor do que a verossimilhança do modelo que a mantêm em sua forma inicialmente contínua, $\left[L_{\text {cont }}(\boldsymbol{\beta})\right]$, em que os vetores $\boldsymbol{\beta}^{*}$ e $\boldsymbol{\beta}$ são obtidos ao maximizar a verossimilhança parcial dos modelos categorizado e contínuo, respectivamente (Vinh-Hung et al. (2009) e Maxwell e Delaney (1993)).

Seja $\boldsymbol{X}=\left(X_{1}, X_{2}, \ldots, X_{m}\right)^{\prime}$ o vetor de covariáveis do modelo de Cox. Suponha, sem perda de generalidade, que $X_{1}$ é a variável contínua que deseja-se categorizar. Se $m>1$, o modelo deve ser inicialmente ajustado na presença das variáveis em suas unidades de medida originais, ou seja, as variáveis contínuas devem ser mantidas em sua forma contínua. A verossimilhança desse modelo, $L_{\text {cont }}(\boldsymbol{\beta})$, deve ser retida. Se $m=1$, a verossimilhança $L_{\text {cont }}(\boldsymbol{\beta})$ será igual a verossimilhança para um modelo univariado ajustado apenas pela variável a ser categorizada, ainda em sua forma contínua.

Como no MPMIN, uma porcentagem central dos valores de $X_{1}$ (entre $80 \%$ e $90 \%$ das observações, caso não haja evidência gráfica para outro intervalo) deve ser testada como possíveis pontos de corte, evitando os extremos para reduzir a chance da formação de grupos muito pequenos. Suponha que deseja-se dividir os valores de $X_{1}$ em $r$ grupos, então para cada possível vetor de pontos de corte $\boldsymbol{c}^{\prime}=\left(c_{1}, \ldots, c_{r-1}\right)$, a verossimilhança parcial do modelo na presença da variável categorizada por $\boldsymbol{c}$, denotada por $L_{c a t}^{c}(\boldsymbol{\beta})$, é calculada.

$\mathrm{O}$ vetor de pontos escolhido como o vetor ideal é aquele associado ao valor de $\boldsymbol{c}$ que minimiza a diferença negativa (isto é, o valor negativo mais próximo de zero) dada por

$$
\Delta^{c}=L_{c a t}^{c}\left(\boldsymbol{\beta}^{*}\right)-L_{\text {cont }}(\boldsymbol{\beta}),
$$

isto é, o vetor que provoca a menor perda de informação referente à categorização e, consequentemente, resulta no modelo mais próximo do modelo que conserva a variável em sua forma contínua.

Alternativamente, o logaritmo das verossimilhanças pode ser usado para o cálculo de 
$\Delta^{c}$, representado por $l$, mas algumas precauções devem ser tomadas. Observe que os valores das verossimilhanças pertencem ao intervalo $[0,1]$, então os logaritmos retornarão valores negativos. Assim como em (3.6), o vetor $\boldsymbol{c}$ escolhido será aquele referente ao maior valor negativo oriundo da expressão equivalente a (3.6), dada por

$$
\Delta^{c}=l_{\text {cat }}^{c}\left(\boldsymbol{\beta}^{*}\right)-l_{\text {cont }}(\boldsymbol{\beta}),
$$

Note que, na prática, (3.7) pode assumir valores negativos e positivos, embora, conceitualmente, espera-se que $l_{\text {cat }}$ seja inferior ao $l_{\text {cont }}$ devido à perda de informação provocada pela categorização da variável $X_{1}$ e, portanto, a diferença devesse apresentar apenas valores negativos (Vinh-Hung et al. (2009) e Maxwell e Delaney (1993)). Os valores positivos para $\Delta^{c}$ podem ocorrer, por exemplo, quando a relação entre a variável $X_{1}$ e o logaritmo do risco é não-linear e não foi avaliada outra forma funcional para melhorar o ajuste do modelo. Nesse caso, pode ocorrer de $l_{\text {cat }}$ apresentar valores superiores ao de $l_{\text {cont }}$.

Supondo um modelo bem ajustado e assumindo que apenas as diferenças negativas devem ser retidas para avaliação do melhor vetor de pontos de corte associado, o uso da fórmula alternativa (3.7) faz sentido. Note que, diferenças positivas e negativas não podem ser comparadas pelo uso da fórmula alternativa, veja explicação no Apêndice B. Apenas a título de comparação, o vetor $\boldsymbol{c}$ associado à menor diferença positiva também será avaliado. Este vetor de pontos de corte está associado ao modelo com limiar que apresenta resultados superiores ao modelo que mantém a variável contínua, mas ainda assim, fornece resultados próximos a ele.

A fim de estimar a estabilidade dos resultados e identificar pontos de corte que sejam robustos à variabilidade dos dados, Vinh-Hung et al. (2009) sugere o uso do método de reamostragem bootstrap. Suponha um banco de dados com $n$ observações, cada amostra bootstrap é obtida ao amostrar do banco de dados original, com reposição, o vetor completo de informações sobre o indivíduo, ou seja, o tempo observado (de falha ou censura), o indicador de falha e o vetor de todas as covariáveis (Gong, 1986). São feitas $B$ reamostragens, cada amostra de tamanho $n$, e em cada uma delas um ponto (ou vetor de pontos) de corte $\boldsymbol{c}_{\text {boot }}$ é estimado pelo MDV. O valor que se repetir mais vezes entre as amostras bootstrap é selecionado como ponto de corte ideal. O valor de B sugerido por Vinh-Hung et al. (2009) é de 10000 .

\subsubsection{Testes corrigidos}

A fim de preservar a taxa de erro do tipo I global e corrigir o consequente viés causado pela seleção do ponto de corte "ótimo" baseada em testes múltiplos alguns testes corrigidos são propostos pela literatura. As primeiras duas correções são propostas quando a análise é feita no cenário univariado e a terceira estatística corrigida permite o ajuste de outras variáveis no modelo. Todos os testes são aplicados no caso de dicotomia (Klein e Wu, 2004) e faz-se uso das definições para o modelo de Cox indicadas no início deste Capítulo. Correções 
para politomia são apresentadas no capítulo seguinte.

\section{Cenário univariado}

Para os testes realizados no modelo ajustado apenas pela variável a ser dicotomizada $X$, suponha o teste $H_{0}: \beta_{c}=0$ ou, equivalentemente, $H_{0}: P(T \leq t \mid X \leq c)=P(T \leq t \mid X>c)$, para todo $t \geq 0$, em que $T$ é a variável aleatória representando o tempo e $c$ é ponto de corte que dicotomiza $X$. Além disso, a informação observada para o indivíduo $i$ da amostra é representada por $\left(t_{i}, \delta_{i}, x_{i}\right)$, em que $t_{i}$ é o instante de falha ou censura observado, $\delta_{i}$ é o indicador de falha e $x_{i}$ é o valor observado da variável $X_{i}, i=1, \ldots, n$. A primeira correção, apresentada por Jespersen (1986), é baseada na estatística

$$
S_{J}=\frac{\sup _{c \in\left[X_{(1)}, X_{(n)}\right]}\left|S_{c}\right|}{\sqrt{D}},
$$

em que $X_{(i)}$ representa a estatística de ordem da variável $X, S_{c}$ é a estatística de teste Score definida em (A.8), avaliada sob $H_{0}$ e com a variável $X$ dicotomizada com relação ao ponto de corte $c$, e $D$ é o número de falhas observadas na amostra. Sob hipótese nula, Jespersen (1986) demonstrou que $S_{J}$ converge em distribuição para o $\sup \left|W^{0}(p)\right|, 0 \leq p \leq 1$, em que $W^{0}$ é uma ponte Browniana definida no Apêndice B em (B.2).

Billingsley (1968) apresentou algumas propriedades de ponte Browniana, em particular, deduziu a distribuição do valor extremo de uma ponte Browniana $\sup \left|W^{0}(p)\right|$, para $0 \leq p \leq 1$ (páginas 83 a 86). A partir desse resultado é possível encontrar valores- $p$ e valores críticos do teste através da expressão

$$
P\left(\sup _{0 \leq p \leq 1}\left|W^{0}(p)\right| \geq b\right)=2\left(\sum_{j=1}^{\infty}(-1)^{j+1} \exp \left[-2 j^{2} b^{2}\right]\right), \quad b>0 .
$$

A soma infinita em (3.8) converge rapidamente, de tal forma que usualmente bastam os 5 primeiros termos para se obter uma aproximação satisfatória. De forma alternativa, Contal e O'Quigley (1999) sugerem aproximar a probabilidade em (3.8) pelo primeiro termo da soma, isto é, $2 \exp \left(-2 b^{2}\right)$, para $b>1$.

Contal e O'Quigley (1999) propuseram uma segunda estatística corrigida de teste, também baseada na estatística Score. Suponha uma amostra de variáveis aleatórias $\phi_{1}, \ldots, \phi_{n}$ permutáveis, ou seja, sua distribuição conjunta é invariante a permutações para qualquer valor de $n$. Se $\phi_{i}$ satisfaz as três condições

$$
\sum_{i=1}^{n} \phi_{i} \underset{p}{\rightarrow} 0, \quad \sum_{i=1}^{n} \phi_{i}^{2} \underset{p}{\rightarrow} 1 \text { e } \max _{1 \leq i \leq n}\left|\phi_{i}\right| \underset{p}{\rightarrow} 0 \quad \text { quando } \quad n \rightarrow \infty
$$


então, sob $H_{0}$, a função aleatória $S_{n}$ definida por

$$
S_{n}(p)=\sum_{i=1}^{[n p]} \phi_{i} \Longrightarrow W^{0}(p)
$$

em que $[n p]$ é o menor inteiro maior do que $(n p-1), p \in[0,1], S_{n}(p)=0$ para $0 \leq p<1 / n$ e o símbolo $\Longrightarrow$ indica convergência fraca. A terceira condição em (3.9) é denominada condição de Noether. O maior interesse do teste corrigido proposto por Contal e O'Quigley (1999) é encontrar o valor $p$ associado ao máximo da estatística $\left|S_{n}(p)\right|$; tal quantidade ao ser multiplicada por $n$ indicará o posto correspondente ao ponto de corte $c$ a ser escolhido. Sob hipótese nula, a distribuição limite de $\max \left|S_{n}(p)\right|$ é a mesma distribuição da estatística de teste corrigida $S_{J}$, dada por (3.8).

Para dados de sobrevivência, o processo $S_{n}(p)$ é alterado para cada valor observado da variável $X$ em que um indivíduo falha. Os scores $\phi_{i}$ são baseados na raiz do numerador da estatística de teste de logrank (3.10) ou, equivalentemente, baseado no teste de Savage. Suponha $D$ tempos de falha ordenados $t_{1}<\ldots<t_{D}$, em uma amostra de tamanho $n$. Para cada possível ponto de corte $c$, os dados podem ser apresentados em $D$ tabelas de contingência $2 \times 2$ associadas a $t_{j}, j=1, \ldots, D$, como apresentado na Tabela 3.2. A raiz do numerador do teste logrank apresentada em (3.3), para um ponto de corte $c$ fixado, é dado por

$$
U(c)=\sum_{j=1}^{D} \phi_{j}=\sum_{j=1}^{D}\left(d_{2 j}-d_{j} \frac{n_{2 j}}{n_{j}}\right),
$$

em que $d_{2 j}$ é o número de falhas no tempo $t_{j}^{-}$no grupo de indivíduos em que $X>c, d_{j}$ é o número de falhas no tempo $t_{j}^{-}, n_{2 j}$ é o número de indivíduos em risco no tempo $t_{j}^{-}$no grupo em que $X>c$ e $n_{j}$ é o total de indivíduos em risco em $t_{j}^{-}$. Note que, no cenário univariado quando duas curvas de sobrevivência são comparadas, o score (3.10) é igual ao vetor score dado por (A.3).

Quando não há censuras, $n=D$ e o teste de Savage é dado por

$$
S_{\text {Sav }}=\sum_{i=1}^{D}-I\left[X^{(i)} \leq c\right] a_{i}
$$

em que $X^{(i)}$ é o valor de $X_{i}$ associado ao tempo de falha ordenado $t_{i}$ e

$$
a_{i}=1-\sum_{k=1}^{i} \frac{1}{D-k+1}
$$

é o score associado ao teste de Savage, em que o termo $\sum_{k=1}^{i} 1 /(D-k+1)$ representa o valor esperado da $i$-ésima estatística de ordem de uma amostra de tamanho $n$ de uma distribuição exponencial padrão (Koziol e Petkau, 1978). Segundo Klein e Wu (2004), sob 
$H_{0}$, a média dos $a_{i}$ 's é igual a zero e a variância é dada por

$$
\sigma^{2}=\frac{\sum_{j=1}^{D} a_{j}^{2}}{D-1}
$$

Koziol e Petkau (1978) demonstraram a igualdade entre o score (3.10) e o negativo do teste de Savage $-S_{\text {Sav }}(3.11)$ :

$$
\begin{aligned}
U(c) & =\sum_{j=1}^{D}\left(d_{2 j}-d_{j} \frac{n_{2 j}}{n_{j}}\right) \\
& =\sum_{j=1}^{D}\left(I\left[X^{(j)} \leq c\right]-\frac{\sum_{i=1}^{D} I\left[X^{(i)} \leq c\right]-\sum_{i=1}^{j-1} I\left[X^{(i)} \leq c\right]}{D-j+1}\right) \\
& =\sum_{j=1}^{D}\left(I\left[X^{(j)} \leq c\right]-\frac{\sum_{i=j}^{D} I\left[X^{(i)} \leq c\right]}{D-j+1}\right) \\
& =\sum_{j=1}^{D} I\left[X^{(j)} \leq c\right]-\sum_{j=1}^{D} I\left[X^{(j)} \leq c\right]\left(\sum_{k=1}^{i} \frac{1}{D-k+1}\right) \\
& =\sum_{j=1}^{D} I\left[X^{(j)} \leq c\right] a_{j}=-S_{\text {Sav }} \quad \mathrm{e},
\end{aligned}
$$

portanto, a igualdade entre os testes logrank e Savage.

Agora, considere $X_{(1)}<\ldots<X_{(D)}$ as estatísticas de ordem da variável $X$. A forma padronizada dos scores do teste de Savage dada por

$$
\phi_{i}=\frac{a_{i}}{\sigma \sqrt{(D-1)}}, i=1, \ldots, D
$$

satisfaz as condições (3.9), isto é,

$$
\begin{gathered}
\sum_{j=1}^{D} \phi_{j}=\sum_{j=1}^{D} \frac{1}{\sqrt{D-1}} \frac{\left(a_{j}-\bar{a}\right)}{\sqrt{\sigma^{2}}}=\frac{1}{\sqrt{D-1}} \frac{\left(\sum_{j=1}^{D} a_{j}-D \bar{a}\right)}{\sqrt{\sigma^{2}}}=0 \\
\sum_{j=1}^{D} \phi_{j}^{2}=\sum_{j=1}^{D}\left[\frac{1}{\sqrt{D-1}} \frac{\left(a_{j}-\bar{a}\right)}{\sqrt{\sigma^{2}}}\right]^{2}=\frac{1}{\sigma^{2}} \frac{\sum_{j=1}^{D}\left(a_{j}-D \bar{a}\right)^{2}}{D-1}=1
\end{gathered}
$$

em que $\bar{a}$ é a média dos scores. Assumindo válida a condição de Noether, então, na ausência de censuras, o processo $S_{n}(p)$ é dado por:

$$
S_{n}(p)=\frac{1}{\sigma \sqrt{D-1}} \sum_{j=1}^{[D p]} a_{j}=\frac{1}{\sigma \sqrt{D-1}} U\left(X_{[D p]}\right)
$$

em que $X_{[D p]}$ é o valor de $X$ no posto $D p$.

No caso em que há censura, ou seja, $n \neq D$, a raiz do numerador da estatística logrank 
é utilizado como score sendo padronizado pela média e variância dos scores do teste de Savage calculadas sobre as observações que falharam, ficando assim definida como

$$
S_{n}(p)=\frac{1}{\sigma \sqrt{D-1}} U\left(X_{[D p]}\right)
$$

em que $\sigma$ é a raiz quadrada da variância apresentada em (3.13). Sob a suposição de um modelo com censura aleatória e sob $H_{0}$, o máximo do módulo dos processos (3.14) e (3.15), $\max \left|S_{n}(p)\right|$, converge em distribuição para o supremo de uma ponte Browniana, sup $\left|W^{0}(p)\right|$, para $0 \leq p \leq 1$, dada por (3.8). O valor de $c$ escolhido como melhor ponto de corte é aquele associado ao posto $D p$ encontrado ao maximizar $\left|S_{n}(p)\right|$, que possui a mesma distribuição limite que a estatística de Jespersen dada por (3.8) (Contal e O’Quigley, 1999).

\section{Cenário multivariado}

A extensão da abordagem proposta por Contal e O'Quigley (1999) permite a inclusão de covariáveis no modelo de regressão além da variável $X$ a ser categorizada. Esta abordagem aplica uma correção aos resíduos baseados no ajuste dessas outras covariáveis representadas por $\boldsymbol{Z}$. Para testar $H_{0}: P(T \leq t \mid X \leq c, \boldsymbol{Z})=P(T \leq t \mid X>c, \boldsymbol{Z})$, para todo $t \geq 0$, suponha o modelo de Cox com sua função de risco dada por

$$
h(t \mid X, \boldsymbol{Z})=h_{0}(t) \exp \left\{\boldsymbol{\beta}^{*^{\prime}} \boldsymbol{Z}+\beta I[X \leq c]\right\}
$$

Para estimar o parâmetro $c$ é necessário, primeiramente, estimar o coeficiente de regressão $\boldsymbol{\beta}^{*}$, por $\boldsymbol{b}^{*}$ ao maximizar a verossimilhança parcial no modelo sem $X$ e, então, calcular os resíduos de Cox-Snell definidos por:

$$
R_{i}=\hat{\Lambda}_{0}\left(t_{i}\right) \exp \left\{\boldsymbol{b}^{*^{\prime}} \boldsymbol{Z}_{\boldsymbol{i}}\right\}
$$

em que $\hat{\Lambda}_{0}\left(t_{i}\right)$ é a função de taxa de falha acumulada estimada pelo estimador de Breslow definido em (3.2). Se o modelo for adequado, esses resíduos se comportam como uma amostra censurada de uma exponencial padrão (Colosimo e Giolo, 2006).

Agora, com os dados ordenados de forma crescente segundo a variável $X$, seja $U_{\beta^{*}}^{c}$ o vetor score, como definido em (A.3), avaliado em $\beta=0, \boldsymbol{\beta}^{*}=\boldsymbol{b}^{*}$ e $c$ o valor de $X$ que separa a variável contínua em dois grupos. Assim, para uma amostra de tamanho $n$, é possível mostrar que

$$
U_{\beta^{*}}^{c}=\sum_{i=1}^{n} I\left[X_{i} \leq c\right] \psi_{i},
$$

em que $\psi_{i}=R_{i}-\delta_{i}$ é o score associado ao $i$-ésimo valor de $X$ ordenado. Consequentemente, 
o ponto de corte $c$ é igual ao $[n p]$-ésimo menor valor de $X$, tal que

$$
U_{\beta^{*}}^{c}(p)=\sum_{i=1}^{[n p]} \psi_{i}
$$

Por meio dos resultados deduzidos por Billingsley (1968) sobre processos ergódicos para somas parciais é possível mostrar que, sob $H_{0}$, o processo

$$
S(p)=\frac{U_{\beta^{*}}^{c}(p)}{v \sqrt{n}}
$$

converge fracamente para um movimento Browniano no intervalo unitário, sendo $v=E\left(\psi_{i}^{2}\right)^{1 / 2}$. Os $\psi_{i}$ 's têm média zero e $v$ pode ser consistentemente estimada por $\sum \psi_{i}^{2} / n$. Desse modo,

$$
S(p)=\frac{\sum_{i=1}^{[n p]} \psi_{i}}{\sqrt{\sum_{i=1}^{n} \psi_{i}^{2}}} .
$$

Uma vez que $\sum \psi_{i}=0$, tem-se que $S(p)-p S(1)=S(p)$ e, então, sob $H_{0}, S(p)$ converge para uma ponte Browniana segundo os resultados de Billingsley (1968) e o valor máximo de $|S(p)|$ tem distribuição limite dada pelo supremo de uma ponte Browniana e, portanto, o valor- $p$ pode ser encontrado usando (3.8). O valor de $p$ que maximiza a função $|S(p)|$ fornece a estimativa para $c$ igual ao $[n p]$-ésimo menor valor de $X$.

\subsubsection{Outros métodos}

Os métodos citados nesta seção não se enquadram no principal objetivo do trabalho (tricotomizar variáveis contínuas em análise de sobrevivência) e, portanto, não são aplicados aos dados e são brevemente descritos.

\section{Seleção de pontos de corte para equações de estimação generalizadas}

Para o caso em que deseja-se dicotomizar uma variável explicativa contínua e assumindo o cenário de Modelos Lineares Generalizados (MLG), Tunes-da-Silva e Klein (2011) propõem dois testes para seleção de ponto de corte: Wald e Score generalizado, e dois métodos para correção da significância dos testes a fim de preservar a taxa do erro tipo I: método de Lausen92, que será descrito no capítulo seguinte, e a segunda abordagem é uma modificação do método de Contal e O'Quigley (1999) também apresentado no capítulo seguinte. Tem-se o interesse em aplicar essas técnicas em problemas de regressão com pseudo-observações.

A abordagem das pseudo-observações é uma técnica flexível para modelagem direta de medidas de sobrevivência em dados com censura à direita, como a função de sobrevivência, a função de incidência acumulada, o tempo de sobrevivência médio para probabilidades de multiestados e a qualidade de vida média (Klein et al., 2008). As pseudo-observações são obtidas por meio da diferença entre o estimador baseado na amostra completa e o estimador 
leave-one-out para a medida de sobrevivência em questão.

Após a estimação das pseudo-observações, o problema envolvendo dados censurados é reduzido ao problema de GEE e os pseudo-valores estimados são usados no modelo de Equações de Estimação Generalizadas (GEE, generalized estimating equations) para modelar o efeito da covariável dicotomizada a partir de uma variável contínua sobre a resposta de interesse.

\section{Métodos de seleção de pontos de corte baseados nas curvas ROC}

Rota et al. (2015) apresentam a extensão dos métodos de estimação de pontos de corte baseados na curva ROC (Receiver Operating Characteristic) para o caso em que a variável resposta é o tempo de falha censurado: (i) Youden Index, (ii) Probabilidade de Concordância e (iii) ponto mais próximo de $(0,1)$. Os métodos (i), (ii) e (iii) são comumente usados no caso de resposta binária, em que deseja-se estimar o ponto de corte $c$ de uma variável contínua $X$, em que os indivíduos com valores de $X$ acima de $c$ (ou abaixo) são classificados como, por exemplo, doentes e, para $X \leq c$, não doentes (saudáveis). Para resposta dicotômica, os métodos citados são funções de $c$ e baseiam-se nas definições de sensibilidade $(S e)$ e especificidade $(S p)$ dadas por:

$$
\begin{aligned}
& S e(c)=P(X>c \mid \text { indivíduo doente }) \quad \text { e } \\
& S p(c)=P(X \leq c \mid \text { indivíduo saudável }) .
\end{aligned}
$$

A extensão das definições de $S e$ e $S p$ para resposta censurada não é direta pelo fato de não ser possível definir se o indivíduo censurado deve ser considerado como doente ou saudável até um determinado instante de tempo e, portanto, $S e$ e $S p$ não podem ser estimadas pelas simples proporções como no caso de resposta binária. Dessa forma, suponha que o tempo de falha seja representado pela variável $Z$, e $\tau$ é um instante de tempo limite de interesse clínico. A definição de doente depende se $Z \leq \tau$ ou $Z>\tau$. Assuma que valores altos de uma variável explicativa $X$ estão relacionados ao aumento do risco de ficar doente. Para um ponto de corte $c$, um indivíduo qualquer é dito como sendo teste positivo ou negativo dependendo se $X>c$ ou $X \leq c$. Dessa forma, em função de $c$, Se e $S p$ são definidor por

$$
\begin{aligned}
& S e(c)=P\left(X>c \mid Z_{i} \leq \tau\right) \quad \mathrm{e} \\
& S p(c)=P\left(X \leq c \mid Z_{i}>\tau\right)
\end{aligned}
$$

representando a probabilidade de um teste dar positivo dado que o indivíduo é doente e a probabilidade de um teste dar negativo dado que o indivíduo é saudável, respectivamente.

A curva ROC é definida ao plotar $S e(c)$ versus $1-S p(c)$, variando o valor de $c$. Os 
métodos Youden Index $(J)$ e Probabilidade de Concordância $(C Z)$ são definidos por

$$
\begin{aligned}
J & =S e(c)+S p(c)-1 \quad \mathrm{e} \\
C Z & =S e(c) \cdot S p(c),
\end{aligned}
$$

e os valores de $c$ que maximizam cada função são escolhidos como os pontos de corte ótimos.

O método do ponto mais próximo a $(0,1)$ é definido como a distância do par (1 $S p(c), S e(c))$ ao ponto de corte ótimo $(0,1)$ no plano ROC, que é obtida ao calcular a distância Euclidiana

$$
E R(c)=\left[(1-S e(c))^{2}+(1-S p(c))^{2}\right]^{1 / 2}
$$

O valor de $c$ que minimiza a função $E R$ é escolhido como ponto de corte ótimo.

Como exemplo de outros métodos, Heagerty e Zheng (2005) propõem uma nova medida de acurácia preditiva de um modelo de sobrevivência baseada na extensão das definições de sensibilidade e especificidade para variáveis dependentes do tempo. Ruopp et al. (2008) propõem, para resposta binária, um método empírico não paramétrico e uma abordagem paramétrica baseada na máxima verossimilhança para o cálculo do Youden Index e estimação do ponto de corte, no caso de dicotomia, a partir de observações afetadas por um limite de detecção (LOD, Limit of Detection); a presença de LOD permite a inserção de dados censurados na estimação do ponto de corte que maximiza a função Youden Index. Nakas et al. (2010) propõem uma generalização do Youden Index de modo a tricotomizar uma variável explicativa contínua. 


\section{Capítulo 4}

\section{Métodos de validação de pontos de corte e de correção do valor- $p$ e do risco relativo}

O valor- $p$ global e os riscos relativos estimados após a aplicação dos métodos de seleção do parâmetro de limiar $\boldsymbol{c}$ baseados em testes múltiplos, usualmente, apresentam um viés e, por isso, alguns métodos para correção dessas medidas foram desenvolvidos. Além disso, mesmo que técnicas de correção sejam aplicadas às estatísticas estimadas, o modelo pode não apresentar valor clínico, na prática. Dessa forma, além das correções, outra questão importante ao derivar um modelo preditivo é verificar se é possível predizer a resposta de pacientes que não pertencem ao conjunto de dados no qual o modelo foi construído. (Lausen e Schumacher (1996), Schumacher et al. (1997), Hothorn e Lausen (2002) e Klein e Wu (2004)).

Qualquer modelo de regressão ajustado deve mostrar-se útil antes de ser utilizado de maneira preditiva ou discriminatória. Por isso a importância da aplicação de técnicas de validação (ou generalização) nos resultados encontrados (Altman e Royston, 2000).

"Usefulness is determined by how well a model works in practice, not by how many zeroes there are in the associated p-values." Douglas G. Altman e Patrick Royston.

\subsection{Validação de resultados}

O método citado por Vinh-Hung et al. (2009) para validação dos pontos de corte, apresentado na Seção 3.3.2, pode ser usado para validar pontos de corte estimados por qualquer método e, também, validar o valor-p e os riscos relativos estimados no modelo categorizado. Suponha que o método $M$ seja utilizado para seleção do vetor de pontos de corte $\boldsymbol{c}$. Para um banco de dados com $n$ observações, são feitas $B$ amostras bootstrap de mesmo tamanho do vetor completo de informações sobre o indivíduo, com reposição.

Em cada uma das amostras, o vetor de pontos de corte $\boldsymbol{c}_{b o o t}$ é estimado pelo método $M$ e os correspondentes riscos relativos e valor- $p$ são calculados a partir do modelo categorizado por $\boldsymbol{c}_{\text {boot }}$. Os valores de $\boldsymbol{c}_{b o o t}$, valor- $p$ e riscos relativos que se repetirem mais vezes entre as amostras bootstrap são selecionados como as correções para as medidas avaliadas. O valor de $B$ sugerido por Vinh-Hung et al. (2009) é de 10000, mas pode ser escolhido ou- 
tro valor que seja considerado grande o suficiente de acordo com o banco de dados em análise.

\subsection{Correções para o valor- $p$ no caso de dicotomia}

Métodos para correção do valor- $p$ são comumente referenciados em artigos sobre categorização de variáveis contínuas (Lausen e Schumacher (1992), Hilsenbeck e Clark (1996), Hothorn e Lausen (2002), Hothorn e Lausen (2003) e Hothorn e Zeileis (2008)). Os dois métodos mais citados são detalhados a seguir.

Os métodos de ajuste sugeridos nesta seção são aplicáveis aos valores- $p$ mínimos $\left(p_{\min }\right)$ encontrados via MPMIN no cenário univariado para o caso de dicotomia.

\subsubsection{Método de Lausen92}

Miller e Siegmund (1982) deduziram a distribuição assintótica da raiz quadrada da estatística $\chi^{2}$ máxima selecionada na busca do ponto de corte "ótimo", para o caso de dicotomia e variável resposta binária. Consequentemente, deduziram uma forma corrigida para o valor- $p$ associado ao teste que maximiza uma estatística $\chi^{2}$. Lausen e Schumacher (1992) demonstraram que tal resultado também poderia ser aplicado às estatísticas calculadas a partir de dados de sobrevivência, além de outras estatísticas de teste com distribuição $t$-student ou Normal.

Para demonstração dos resultados encontrados por Lausen e Schumacher (1992), suponha independência e mesma distribuição para os pares $\left(T_{1}, \delta_{1}\right), \ldots,\left(T_{n}, \delta_{n}\right)$, em que $T_{i}$ são os tempos observados de falha ou censura e $\delta_{i}$ é o indicador de falha, para $i=1, \ldots, n$. Além disso, sendo $X$ uma variável aleatória contínua, suponha que deseja-se testar a hipótese nula de independência entre a variável a ser categorizada $X$ e o par $(T, \delta)$, ou seja, para todo $c$

$$
H_{0}: P(T \leq t \mid X \leq c)=P(T \leq t \mid X>c), \text { para todo } t \geq 0,
$$

em que $c$ é o ponto de corte da variável $X$. Sejam $R_{1 n}, \ldots, R_{n n}$ os postos de $T_{1}, \ldots, T_{n}$ e $a_{n}(1), \ldots, a_{n}(n)$ representam scores convenientemente definidos. No caso de empate ou censura nas observações, $a_{n}(i)$ refere-se aos scores médios ou à estatística logrank, $i=1, \ldots, n$. Dessa forma, seja

$$
S_{n}(c)=\sum_{i=1}^{n} I\left[X_{i} \leq c\right] a_{n}\left(R_{i n}\right)=\sum_{I\left[X_{i} \leq c\right]} a_{n}\left(R_{i n}\right)
$$

uma estatística de postos para $c$ fixado (e desconhecido), em que as amostras são definidas por $c$, e $I$ é a função indicadora. Para estimação de $c$ e avaliação da hipótese nula correspondente, o teste estatístico proposto pelos autores utiliza o máximo do módulo da forma padronizada da estatística de postos, dado por:

$$
M_{n}\left(\epsilon_{1}, \epsilon_{2}\right)=\max _{c \in\left[x_{1}, x_{2}\right]}\left|T_{n}(c)\right|
$$


em que,

$$
\begin{gathered}
T_{n}(c)=\frac{S_{n}(c)-E\left(S_{n}(c) \mid a, X\right)}{\left(\operatorname{Var}\left(S_{n}(c) \mid a, X\right)\right)^{1 / 2}}, \\
E(S n(c) \mid a, X)=n F_{n X}(c) \bar{a}_{n}, \mathrm{e} \\
\operatorname{Var}(\operatorname{Sn}(c) \mid a, X)=A_{n}^{2} n F_{n X}(c)\left(1-F_{n X}(c)\right),
\end{gathered}
$$

para $F_{n X}(c)=(1 / n) \sum_{i=1}^{n} I\left[X_{i} \leq c\right]$ a função de distribuição empírica de $X, x_{1}=F_{n X}^{-1}\left(\epsilon_{1}\right)$, $x_{2}=F_{n X}^{-1}\left(\epsilon_{2}\right), 0<\epsilon_{1}<\epsilon_{2}<1$ e a média e a variância dos scores $a_{i}$ 's são dadas, respectivamente, por $\bar{a}_{n}=(1 / n) \sum_{i=1}^{n} a_{n}(i)$ e $A_{n}^{2}=[1 /(n-1)] \sum_{i=1}^{n}\left[a_{n}(i)-\bar{a}_{n}\right]^{2}, i=1, \ldots, n$. O intervalo $\left[\epsilon_{1}, \epsilon_{2}\right]$ indica os valores de $X$ usados na busca ao parâmetro de limiar $c$, ou seja, $c \in\left[F_{n X}^{-1}\left(\epsilon_{1}\right), F_{n X}^{-1}\left(\epsilon_{2}\right)\right]$, em que $F_{n X}^{-1}(t)=\min \left\{x: F_{n X}(x) \geq t\right\}$. Por meio da restrição dos valores de busca da variável $X$ espera-se garantir uma quantidade razoável de observações em ambos os grupos e permitir os argumentos assintóticos usados para convergência da distribuição.

Assim, o estimador do ponto de corte $\hat{c}$ é dado por:

$$
\hat{c}=\min \left\{c: c \in\left[F_{n X}^{-1}\left(\epsilon_{1}\right), F_{n X}^{-1}\left(\epsilon_{2}\right)\right],\left|T_{n}(c)\right|=M_{n}\left(\epsilon_{1}, \epsilon_{2}\right)\right\} .
$$

É comum que mais de um valor de $k \in\{1, \ldots, n-1\}$ forneça o valor máximo de $\left|T_{n}(c)\right|$ e, portanto, o mínimo restringe à uma única solução para verificar o estimador de $\hat{c}$. Usando resultados gerais para variáveis aleatórias permutáveis de Billingsley (1968), Lausen e Schumacher (1992) demonstraram que a distribuição de $M_{n}\left(\epsilon_{1}, \epsilon_{2}\right)$, quando $n \rightarrow \infty$, converge para a mesma distribuição assintótica da raíz quadrada de uma $\chi^{2}$ deduzida por Miller e Siegmund (1982), dada por

$$
P\left(\sup _{t \in[\epsilon, 1-\epsilon]} \frac{\left|W^{0}(t)\right|}{(t(1-t))^{1 / 2}} \leq b\right) \cong 1-\varphi(b)\left(b-\frac{1}{b}\right) \log \left(\frac{(1-\epsilon)^{2}}{\epsilon^{2}}\right)+4\left(\frac{\varphi(b)}{b}\right)
$$

em que $t=F_{X}(x)$, sendo $F_{X}(x)=\sum_{i=1}^{n} I\left[X_{i} \leq x\right]$ a função de distribuição da variável $X, W^{0}$ representa uma ponte Browniana como definida em (B.2), $\varphi(b)$ denota a função densidade da Normal padrão e $b \rightarrow \infty$. Para dados de sobrevivência é usual tomar os scores da estatística logrank, dados por (3.10). Ao provar que os scores $a_{i}$ 's satisfazem as condições (3.9) e usando propriedades sobre os quantis empíricos e argumentos padrões sobre funções contínuas à direita com valores em [0,1] apresentados em (Billingsley (1968), página 29) verifica-se a convergência desejada.

O processo para dedução da correção para o valor- $p$ de Lausen e Schumacher (1992) se assemelha ao processo de dedução do teste corrigido proposto por Contal e O'Quigley (1999) apresentado na Seção 3.3.3. O processo de Contal e O'Quigley (1999) converge para uma ponte Browniana e pode ser interpretado como um redimensionamento sequencial dos 
incrementos, ou seja, cada parcela do processo é ajustada de modo a seguir as propriedades desejadas, por outro lado, o procedimento sugerido por Lausen e Schumacher (1992) deriva um teste estatístico após uma padronização global (Contal e O’Quigley, 1999).

O nível crítico corrigido para o teste que verifica a influência da variável categorizada sobre o tempo de sobrevivência, sob $H_{0}$, pode ser obtido por meio da expressão

$$
p_{\text {cor } 1}=\varphi(z)\left(z-\frac{1}{z}\right) \log \left(\frac{(1-\epsilon)^{2}}{\epsilon^{2}}\right)+4 \frac{\varphi(z)}{z}
$$

em que $z$ é o quantil $\left(1-p_{\min } / 2\right)$ da distribuição da Normal padrão e $p_{\min }$ é o valor- $p$ obtido pelo MPMIN.

Essa correção é indicada para quando existe um grande número de valores de pontos de corte a serem testados $(\geq 50)$. Para poucos pontos testados, esse método é muito conservador e pode apresentar valores de $p_{c o r 1}$ maiores do que 1, o que não é visto como um problema já que o interesse é buscar valor- $p$ mínimo associado aos testes múltiplos (Hilsenbeck e Clark, 1996).

Altman et al. (1994) deduziram algumas simplificações para a fórmula (4.1). Como, por exemplo, para $\epsilon=0.05$

$$
p_{\text {alt } 5}=-3.13 p_{\min }\left(1+1.65 \log \left(p_{\min }\right)\right)
$$

e para $\epsilon=0.10$

$$
p_{\text {alt } 10}=-1.63 p_{\min }\left(1+2.35 \log \left(p_{\min }\right)\right) .
$$

Essas aproximações trabalham bem quando o $p_{\text {min }}$ encontrado pertence ao intervalo $[0.0001,0.1]$. (Faraggi e Simon, 1996) indicam que, para obtenção de $p_{c o r 1} \leq 0.05$ é necessário obter um $p_{\min } \leq 0.002$.

A importância da correção do valor-p é exemplificada por Holländer et al. (2004) na avaliação do tempo de sobrevivência de pacientes com câncer de mama. A variável prognóstica SPF (S-phase fraction) é comumente usada para avaliação do tempo e, por isso, foi dicotomizada via teste logrank por MPMIN fornecendo $p_{\min }=0.007$, usando como intervalo de busca os quantis de 10 a 90 porcento da distribuição da variável SPF.

O resultado encontrado fornece indícios para acreditar que as curvas dos grupos com valores abaixo e acima do ponto de corte escolhido diferem. Entretanto, o valor- $p$ corrigido por (4.1) resultou em 0.12 , que indica um resultado contrário. A correção pode dar evidências para outra tomada de decisão, como neste caso, que a dicotomia não foi adequada e deve-se investigar outra forma de categorizar ou, ainda, outra forma funcional para covariável.

\subsubsection{Método de Lausen94}

A correção de Bonferroni padrão, frequentemente usada para controlar o erro tipo I global ao corrigir o valor- $p$ de cada um dos testes no caso de comparações múltiplas, diz que o nível 
de significância global é dado por $p_{\text {min }} \times m$, em que $m$ é o número de pontos de corte testados ou, equivalentemente, de testes realizados (Colosimo e Giolo, 2006). Essa abordagem é válida somente no caso em que as consecutivas estatísticas de teste são independentes, que não é o caso dos testes realizados via MPMIN.

Uma versão modificada da correção de Bonferroni padrão foi proposta por Lausen et al. (1994) e Altman et al. (1994) e o ajuste feito é baseado na desigualdade de Bonferroni melhorada de Worsley (1982). O valor- $p$ corrigido, denotado por $p_{\text {cor } 2}$, considera a correlação entre as estatísticas de teste para pontos de corte adjacentes. Suponha que $k$ pontos de corte sejam testados, então, segundo Lausen et al. (1994), a correção é dada por

$$
\begin{gathered}
p_{\text {cor } 2}=p_{\text {min }}+\sum_{i=1}^{k-1} D\left(\epsilon_{i}, \epsilon_{i+1}\right), \quad \text { em que } \\
D\left(\epsilon_{i}, \epsilon_{i+1}\right)=\frac{\exp \left(-z^{2} / 2\right)}{\pi}\left[a\left(\epsilon_{i}, \epsilon_{i+1}\right)-\left(\frac{z^{2}}{4}-1\right)\left(\frac{a\left(\epsilon_{i}, \epsilon_{i+1}\right)^{3}}{6}\right)\right] \mathrm{e} \\
a\left(\epsilon_{i}, \epsilon_{i+1}\right)=\left[1-\frac{\epsilon_{i}\left(1-\epsilon_{i+1}\right)}{\left(1-\epsilon_{i}\right) \epsilon_{i+1}}\right]^{1 / 2} .
\end{gathered}
$$

O valor $z$ é definido como na equação (4.1) e $\epsilon_{i}$ é a proporção de valores observados abaixo do $i$-ésimo ponto de corte. Correlações altas, como quando pontos adjacentes definem subgrupos praticamente idênticos, resultam em termos de ajuste menores (Hilsenbeck e Clark, 1996). Ao contrário do método de Lausen92, esta correção trabalha melhor quando poucos pontos de corte são avaliados. Neste caso, $p_{\text {cor } 2}$ tende a apresentar resultados menores do que $p_{\text {cor } 1 \text {. }}$

Mazumdar e Glassman (2000) recomendam o uso do mínimo entre $p_{c o r 1}$ e $p_{c o r 2}$, uma vez que os dois métodos tendem a dar correções muito conservadoras.

\subsubsection{Outras correções}

Hilsenbeck e Clark (1996) sugerem o uso da abordagem do teste de permutação ou de aleatorização para correção da probabilidade de significância. Sob a hipótese nula, os grupos gerados pelo ponto de corte selecionado não são estatisticamente distintos e, portanto, os valores da variável a ser categorizada $X$, podem ser aleatoriamente permutados pela amostra e o MPMIN é aplicado sobre essa nova amostra.

Repetindo esse processo um grande número de vezes e retendo os valores das estatísticas maximais selecionadas é possível gerar uma distribuição nula empírica para distribuição da estatística maximal. Por conseguinte, o valor- $p$ ajustado empiricamente, $p_{\text {emp }}$, pode ser obtido ao comparar a estatística de teste maximal observada com a distribuição nula empírica. Esta abordagem é computacionalmente intensiva e é intuitivamente atrativa por reproduzir o processo de maximização da seleção de ponto de corte.

Hothorn e Lausen (2003) deduziram a distribuição exata da estatística de postos maxi- 
mal selecionada baseada na extensão do algoritmo para a distribuição de uma estatística de postos lineares. Para amostras pequenas ou intermediárias o limite inferior da distribuição exata retorna melhores resultados do que as aproximações baseadas na desigualdade de Bonferroni melhorada ou no processo Gaussiano assintótico. Hothorn e Zeileis (2008) deduziram a estatística maximal selecionada generalizada. Outras formas de correção podem ser encontradas em Hothorn e Lausen (2002).

\subsection{Correções para o risco relativo no caso de dicotomia}

Quando comparações múltiplas são feitas a fim de encontrar o ponto de corte ideal, a correção do nível crítico do teste é importante, mas não avalia a potencial superestimação do efeito da variável dicotomizada $X^{\prime}=I[X \leq c]$ sobre o tempo de sobrevivência $T$, em que $c$ é o ponto de corte estimado. Um modo para corrigir este problema é diminuir a estimativa do parâmetro $\beta$ associado à variável, por meio de um valor $\hat{d}$, denominado fator de contração (shrinkage factor), de tal modo que o risco relativo corrigido deve ser estimado por

$$
\widehat{r r}_{c o r}=\exp (\hat{d} \hat{\beta})
$$

Avaliando o efeito do fator de contração sobre o risco relativo dado por (4.2), observa-se

que valores de $\hat{d}$ próximos a 1 indicam menor grau de superestimação, enquanto que valores próximos a zero indicam que o parâmetro de regressão foi substancialmente superestimado. Schumacher et al. (1997) propuseram quatro formas de estimar o fator de contração no cenário univariado em que a variável categorizada é dividida em apenas dois grupos.

\subsubsection{Fator de contração ad hoc}

A primeira abordagem, ad hoc, determina o fator de contração por meio do valor- $p$ corrigido pelo método de Lausen92, denotado por $p_{\text {cor }}$. Seja $z_{\text {pmin }}^{2}$ o valor da estatística de teste Wald (A.6), sob a hipótese nula, $H_{0}: \beta=0$. O fator de contração, $\hat{d}_{\text {adhoc }}$, é deduzido ao resolver

$$
1-F_{\chi^{2}}\left(z_{\text {pmin }}^{2} d_{\text {adhoc }}^{2}\right)=p_{c o r},
$$

em que $F_{\chi^{2}}$ representa a função de distribuição qui-quadrado com um grau de liberdade. Seja $z_{\text {pcor }}^{2}$ o valor da estatística Wald correspondente ao $p_{c o r}$. Assumindo que o desvio padrão do coeficiente de regressão estimado permanece o mesmo, o fator de contração é dado por

$$
\widehat{d}_{\text {adhoc }}=\sqrt{\frac{z_{\text {pcor }}^{2}}{z_{\text {pmin }}^{2}}} .
$$

As duas próximas abordagens são baseadas em técnicas de reamostragem e, portanto, são computacionalmente intensivas. 


\subsubsection{Fator de contração $v c$}

A segunda forma de deduzir o fator de contração baseia-se no método de calibração de um modelo por meio de validação cruzada $(v c)$ proposto por Verweij e Houwelingen (1993). A fim de exemplificar o processo de estimação de $\hat{d}_{v c}$, suponha o caso de validação cruzada dupla (two-fold cross-validation), em que a variável contínua $X$ é dicotomizada a partir de um ponto de corte $c$ estimado por um método $M$, e a variável binária é representada por $X^{\prime}$.

Inicialmente, a amostra de tamanho $n$ deve ser particionada aleatoriamente em dois grupos disjuntos $\mathrm{A}$ e B, de modo que haja um número similar de indivíduos em cada grupo, denotados por $n_{A}$ e $n_{B}$. Ao maximizar as verossimilhanças parciais para cada grupo obtémse as estimativas dos coeficientes de regressão associados à variável binária $\hat{\beta}_{-B}$ e $\hat{\beta}_{-A}$, respectivamente. Note que $\hat{\beta}_{-A}$ é independente das observações de A pois foi calculado no grupo $\mathrm{B}$, enquanto que $\hat{\beta}_{-B}$ é independente das observações de $\mathrm{B}$.

O score $\left(X_{i}^{\prime}-\bar{X}^{\prime}\right) \hat{\beta}_{-A}$ pode ser usado como um preditor para uma nova observação $T_{i}$ pertencente ao grupo A, em que $i=1,2, \ldots, n_{A}$ e $\bar{X}^{\prime}$ representa a média amostral de $X^{\prime}$. O mesmo pode ser observado para o grupo $\mathrm{B}$, ou seja, $\left(X_{i}^{\prime}-\bar{X}^{\prime}\right) \hat{\beta}_{-B}, i=1,2, \ldots, n_{B}$, funciona como preditor de uma nova observação $T_{i}$ pertencente ao grupo B. Ao concatenar os scores apresentados acima, uma nova variável $\left(X_{i}^{\prime}-\bar{X}^{\prime}\right) \hat{\beta}_{-k}$ é formada, em que $i=1,2, \ldots, n_{A}+n_{B}$ e $k=A$ ou $B$ é o grupo ao qual o $i$-ésimo indivíduo pertence.

Para verificar seu potencial preditivo, a nova variável é incluída como única variável associada ao tempo de sobrevivência observado no modelo de Cox usando todo conjunto de dados. O coeficiente de regressão estimado ao maximizar a verossimilhança parcial do modelo ajustado pela nova variável pode ser usado como fator de contração, $\hat{d}_{v c}$. Consequentemente, o preditor ajustado para o $i$-ésimo paciente é dado por $\left(X_{i}^{\prime}-\bar{X}^{\prime}\right) \hat{d}_{v c} \hat{\beta}_{-k}, i=1,2, \ldots, n_{A}+n_{B}$.

Este processo deve ser repetido dez ou mais vezes, e o fator de contração estimado é definido como a média dos fatores $\hat{d}_{v c}$ encontrados em cada iteração. Observe que a padronização para a variável $X^{\prime}$, dada por $\left(X_{i}^{\prime}-\bar{X}^{\prime}\right)$, é necessária para que a estimação do fator $\hat{d}_{v c}$ seja baseada em todos os coeficientes de regressão estimados, $\hat{\beta}_{-k}$, e não só sobre aqueles associados a $X_{i}^{\prime}=1$.

A validação cruzada tripla, quádrupla ou por $m$ vezes, para $m \in \mathbb{N}$, pode ser aplicada seguindo o mesmo algoritmo descrito acima. Schumacher et al. (1997) avaliaram o caso de validação cruzada deixando um fora ao tomar $m=n$, e o caso por 10 vezes ao tomar $m=10$ (leave-one-out e 10-fold cross-validation) e verificaram que os fatores de contração encontrados por esses dois métodos resultam essencialmente no mesmo valor. Em geral, as médias dos fatores de contração encontrados para diferentes valores de $m$ são similares. Entretanto, a $v c$ dupla é preferível pela simplicidade do algoritmo e não produz uma taxa de erro do tipo I tão acima do valor nominal (Mazumdar et al., 2003). 


\subsubsection{Fator de contração boot}

O primeiro passo para aplicação da terceira abordagem é a geração de $B=100$ amostras bootstrap. Cada uma delas, de mesmo tamanho da amostra original, é obtida ao amostrar, com reposição, o vetor completo de informações sobre a observação (quer dizer, o tempo $T_{i}$, a indicadora de falha $\delta_{i}$ e o vetor de todas as covariáveis). Para cada amostra bootstrap o ponto de corte "ótimo", $c_{\text {boot }}$, é calculado e seu correspondente parâmetro, $\hat{\beta}_{b o o t}$, é estimado. O ponto de corte obtido na amostra bootstrap deve ser aplicado aos dados originais conduzindo à estimação do parâmetro $\tilde{\beta}_{\text {boot }}$.

Espera-se que a média dos parâmetros estimados nos dados originais ao usar os pontos de

corte das amostras bootstrap, $\tilde{\hat{\beta}}_{\text {boot }}$, seja menor do que a média das estimativas nas amostras bootstrap para as quais os pontos de corte foram derivados, $\overline{\hat{\beta}}_{\text {boot }}$ (Schumacher et al., 1997). (Efron e Tibshirani, 1994) propõem estimar a quantidade de superestimação utilizando a diferença $\overline{\hat{\beta}}_{\text {boot }}-\overline{\tilde{\beta}}_{\text {boot }}$. Dessa forma, a estimativa para o risco relativo corrigido pode ser feita ao remover esse termo de superestimação, tal que

$$
\widehat{r r}_{\text {boot } 1}=\exp \left(\hat{\beta}-\left(\overline{\hat{\beta}}_{\text {boot }}-\overline{\tilde{\beta}}_{\text {boot }}\right)\right) \text {. }
$$

Também é possível estimar o fator de contração para correção do risco:

$$
\hat{d}_{\text {boot }}=\frac{\overline{\hat{\beta}}_{\text {boot }}}{\overline{\tilde{\beta}}_{\text {boot }}} .
$$

\subsubsection{Fator de contração heurístico}

A quarta forma de calcular o fator de contração é baseada no estimador heurístico descrito por Van Houwelingen e Le Cessie (1990). Seja $\widehat{\operatorname{var}}(\hat{\beta})$ a variância estimada de $\hat{\beta}$, coeficiente de regressão estimado associado à variável dicotomizada, para um ponto de corte fixado e desprezando a variabilidade adicional causada pela estimação do ponto de corte, o estimador para $\hat{d}$ é dado por

$$
\widehat{d}_{\text {heur }}=\frac{\hat{\beta}^{2}-\widehat{v a r}(\hat{\beta})}{\hat{\beta}^{2}} .
$$

O ponto de corte usado deve ter sido estimado por um dos métodos que utilizam comparações múltiplas, como os métodos do valor- $p$ mínimo e da diferença das verossimilhanças.

\subsection{Correção para valor- $p$ e razão de risco}

Os métodos de validação cruzada e o split-sample são usados para calcular as formas corrigidas do nível de significância e do risco relativo associados ao modelo categorizado (Royston et al., 2006). Estes métodos podem ser aplicados aos casos de dicotomia e politomia nos cenários univariado e multivariado.

É comum a aplicação deles no cenário univariado, mesmo quando o verdadeiro modelo 
possui outras covariáveis. Mazumdar et al. (2003) sugerem a extensão para o caso multivariado. Ademais, a extensão para o caso de politomia é proposto.

\subsubsection{Validação cruzada}

Suponha que o vetor de pontos de corte $\boldsymbol{c}$ foi deduzido por um dos métodos descritos no Capítulo 3, na Seção 3.3, chamado genericamente por $M$. O procedimento é bastante parecido ao método do fator de contração vc; entretanto, a ação realizada dentro dos grupos de treinamento e validação são diferentes.

Para estimar o nível de significância e o risco relativo é necessário dividir a amostra em $k$ grupos mutuamente exclusivos de tamanhos aproximadamente iguais, em que 1 deles é usado para estimação dos pontos de corte $\boldsymbol{c}_{v c}$ (grupo de treinamento) e os outros $k-1$ grupos (grupo de teste ou de validação) são categorizados segundo este ponto; este procedimento é feito para todos os $k$ grupos de modo que, ao final, todas as observações pertençam a uma das categorias formando uma nova variável preditora; o modelo de Cox na presença dessa nova variável é ajustado e estratificado pelos $k$ grupos e a estimação do valor- $p$ e dos riscos relativos associados a $\boldsymbol{c}_{v c}$ é feita.

Faraggi e Simon (1996) sugerem o uso de $k=2$ já que a validação cruzada para valores maiores de $k$ produzem taxas do erro tipo I acima da esperada (usualmente 0.05). Por isso, o algoritmo para $k=2$ é melhor descrito a seguir. A fim de simplificar a notação, será considerado o caso em que apenas um ponto de corte é estimado (caso de dicotomia), entretanto, a extensão para o caso em que $\boldsymbol{c}$ representa um vetor de pontos de corte associada à categorização em três ou mais subgrupos é diretamente alcançada.

i Os dados são divididos aleatoriamente ao meio em dois subconjuntos, A e B;

ii $\mathrm{O}$ ponto de corte $c_{(-B)}$ é estimado ao aplicar o método $M$ no subconjunto A;

iii As observações do subconjunto B são categorizadas segundo o ponto de $\operatorname{corte} c_{(-B)}$ e alocadas nos grupos $\mathrm{H}$ (valores acima do ponto de corte) e L (valores abaixo do ponto de corte);

iv $\mathrm{O}$ ponto de corte $c_{(-A)}$ é estimado ao aplicar o método $M$ no subconjunto B e

v As observações do subconjunto A são categorizadas segundo o ponto de $\operatorname{corte} c_{(-A)}$ e alocadas nos grupos H e L.

Uma vez finalizado o procedimento, todas as observações do conjunto de dados pertencem ao conjunto $\mathrm{H}$ ou $\mathrm{L}$, formando uma nova variável preditora $X^{*}$. Então, o valor- $p$ e o risco relativo são estimados considerando o modelo de regressão de Cox univariado ou multivariado, ajustado na presença de $X^{*}$ e estratificado tomando os subconjuntos A e B como estratos (Royston et al., 2006). 
Devido à chance da divisão aleatória da amostra separar em grupos de modo que poucos eventos sejam observados em cada um deles, embora não haja citação desse procedimento nos artigos, é proposto que o algoritmo da validação cruzada seja repetido $B$ vezes $(B \geq 50)$, para que o valor- $p$ corrigido seja dado pela moda dos valores- $p$ estimados e o risco relativo corrigido seja definido pela moda dos riscos relativos estimados.

Segundo Mazumdar et al. (2003), o ponto chave deste método é que o valor $\boldsymbol{c}$ usado para categorizar cada observação seja selecionado em um subconjunto que exclui essa observação, reduzindo as chances de estimativas viciadas causadas pela prática do ajuste e validação do modelo realizados sobre a mesma amostra. Note que os pontos de corte encontrados durante o procedimento da validação cruzada, $c_{(-A)}$ e $c_{(-B)}$, são irrelevantes na escolha do ponto de corte final a ser utilizado, que é encontrado por um método $M$ avaliado no conjunto de dados original.

\subsubsection{Split-Sample}

Este método é parecido com a validação cruzada. A amostra é particionada em dois subconjuntos de tamanhos aproximadamente iguais, um grupo de treinamento e outro grupo de validação. O ponto (ou vetor de pontos) de corte encontrado por um método $M$ aplicado ao conjunto de treinamento é usado para categorizar os indivíduos do grupo de validação, formando uma nova variável preditora categórica.

O valor- $p$ e os riscos relativos são calculados sob o modelo de Cox univariado ou multivari-

ado ajustado sobre o grupo de validação na presença da variável categorizada. Espera-se que as estimativas encontradas no grupo de validação sejam não viciadas pois o ponto de corte utilizado foi deduzido no conjunto de treinamento (Mazumdar et al. (2003) e Royston et al. (2006)). Assim como no caso da validação cruzada, é proposto repetir esse algoritmo um certo número de vezes $(\geq 50)$ e as medidas corrigidas são dadas pelas modas dos valores- $p$ e riscos relativos estimados.

Este método tende a ser preferível à validação cruzada pela simplicidade e familiaridade, pois costuma ser usado para validação de modelos prognósticos. Entretanto, essa técnica pode não ser tão eficiente em conjuntos de dados pequenos pois os pontos de corte e as medidas de interesse seriam avaliados em apenas metade da amostra (Mazumdar et al., 2003). 


\section{Capítulo 5}

\section{Aplicação}

Neste capítulo é realizada a aplicação dos métodos discutidos aos dados do InCor, apresentados no Capítulo 2. O objetivo é avaliar a discretização da variável fração de ejeção do ventrículo esquerdo (FE) originalmente contínua, importante fator prognóstico para os pacientes com insuficiência cardíaca. O caso de dicotomia será ilustrado, apesar do objetivo principal ser a divisão dos pacientes em três grupos segundo a FE (tricotomia). O método da diferença das verossimilhanças (MDV) e o método do valor- $p$ mínimo (MPMIN) se dividem da seguinte forma:

- Método da diferença das verossimilhanças 1 (MDV1): Seleciona o ponto de corte associado a menor diferença negativa.

- Método da diferença das verossimilhanças 2 (MDV2): Seleciona o ponto de corte associado a menor diferença positiva.

- Método do valor-p mínimo 1 (MPMIN1): Seleciona o ponto de corte associado ao menor valor- $p$ sob o teste razão de verossimilhança.

- Método do valor-p mínimo 2 (MPMIN2): Seleciona o ponto de corte associado ao menor valor- $p$ sob o teste Wald.

- Método do valor- $p$ mínimo 2 (MPMIN3): Seleciona o ponto de corte associado ao menor valor- $p$ sob o teste Score.

Cada um dos métodos anteriores será utilizado sob os cenários:

- Multivariado 1 (M1): A seleção das variáveis explicativas é feita a partir das variáveis previamente categorizadas, exceto a FE que é mantida contínua, e os pontos de corte são selecionados sob o modelo com as variáveis categóricas selecionadas.

- Multivariado 2 (M2): A seleção das variáveis explicativas é feita a partir das variáveis mantidas em suas formas originais (como foram apresentadas no banco de dados) e os pontos de corte são selecionados sob o modelo com as variáveis contínuas selecionadas. 
- Multivariado 3 (M3): A seleção das variáveis explicativas é feita a partir das variáveis mantidas em suas formas originais (como foram apresentadas no banco de dados). Após o ajuste do modelo, as variáveis selecionadas de origem contínua são categorizadas e, então, os pontos de corte são selecionados sob esse novo modelo.

- Univariado (Uni): Os pontos de corte são selecionados sob o modelo ajustado apenas pela variável FE.

Após a seleção serão aplicadas as seguintes técnicas de validação e correção dos valores- $p$ e dos riscos relativos:

- Bootstrap (boot): Valida o ponto de corte selecionado, o valor- $p$ e os riscos relativos estimados a partir do modelo categorizado.

- Validação Cruzada (vc): Corrige valor- $p$ e riscos relativos.

- Lausen92: Corrige valor-p obtido via MPMIN no cenário univariado para o caso de dicotomia.

- Lausen94: Corrige valor-p obtido via MPMIN no cenário univariado para o caso de dicotomia.

- Fator de contração adhoc $\left(f c_{a d h o c}\right)$ : Corrige o risco relativo obtido via MPMIN para o caso de dicotomia.

- Fator de contração heurístico $\left(f c_{\text {heur }}\right)$ : Corrige o risco relativo para o caso de dicotomia.

- Fator de contração boot1 $\left(f c_{b o o t} 1\right)$ : Corrige o risco relativo para o caso de dicotomia.

- Fator de contração boot2 $\left(f c_{\text {boot }} 2\right)$ : Corrige o risco relativo para o caso de dicotomia.

- Fator de contração $\boldsymbol{v} \boldsymbol{c}\left(f c_{v c}\right)$ : Corrige o risco relativo para o caso de dicotomia.

Cada cenário apresenta uma forma distinta de selecionar as variáveis explicativas que tem efeito sobre a variável resposta (tempo até o óbito). Estas foram as variáveis que se apresentaram significativas em pelo menos um dos cenários e suas respectivas categorias propostas pelos médicos do InCor:

Informações demográficas:

- Idade (IDADE) (anos): categorizada em inferior a 40 anos, de 40 a 65 anos, superior a 65 anos;

- Índice de Massa Corpórea (IMC): razão entre o peso e o quadrado da altura $\left(\mathrm{Kg} / \mathrm{m}^{2}\right)$, categorizado em inferior a $25 \mathrm{~kg} / \mathrm{m}^{2}$, de 25 a $30 \mathrm{~kg} / \mathrm{m}^{2}$, superior a $30 \mathrm{~kg} / \mathrm{m}^{2}$ e Sem resposta. 


\section{Informações clínicas:}

- Pressão arterial sistólica (PAS) (mmHg): pressão arterial na contração (sístole) do coração, (mmHg), categorizada como: inferior a $100 \mathrm{mmHg}$, de 100 a $130 \mathrm{mmHg}$, superior a $130 \mathrm{mmHg}$ e Sem resposta;

- Pressão arterial diastólica (PAD) (mmHg): pressão arterial no relaxamento (diástole) do coração (mmHg), cujas categorias consideradas foram: inferior a $80 \mathrm{mmHg}$, de 80 a $90 \mathrm{mmHg}$, superior a $90 \mathrm{mmHg}$ e Sem resposta;

- Etiologia (ETIO): categorizado como chagásico, hipertensivo, isquêmico, outros e Sem resposta;

- Classe funcional de insuficiência cardíaca (CLASSE): categorizado como classe I, classe II, classe III, classe IV e Sem resposta. O estado do paciente piora conforme aumenta a classe funcional;

- Etilismo (ETIL): Leve; moderado; intenso; sim, mas não quantificado; ex-etilista; não e Sem resposta.

Informações laboratoriais:

- Sódio (SODIO) (mEq/L): categorizado em inferior a $136 \mathrm{mEq} / \mathrm{L}, 136 \mathrm{mEq} / \mathrm{L}$ ou mais e Sem resposta;

- Leucócitos (LEUC) $\left(\mathrm{mm}^{3}\right)$ : classificados como inferior a 4000 por $\mathrm{mm}^{3}$, de 4000 a 11000 por $\mathrm{mm}^{3}$, superior a 11000 por $\mathrm{mm}^{3}$ e Sem resposta;

- Triglicérides (TRIGLI) (mg/dL): categorizados em inferior a $150 \mathrm{mg} / \mathrm{dL}$, de 150 a 300 $\mathrm{mg} / \mathrm{dL}$, superior a $300 \mathrm{mg} / \mathrm{dL}$ e Sem resposta;

- Creatinina (CREAT) (mg/dL): categorizada em inferior a $1.3 \mathrm{mg} / \mathrm{dL}$, de 1.3 a 2.6 $\mathrm{mg} / \mathrm{dL}$, superior a $2.6 \mathrm{mg} / \mathrm{dL}$ e Sem resposta.

Informações Morfológicas do coração:

- Espessura do septo (SEPTO) (mm): categorizada em inferior a $8 \mathrm{~mm}$, de 8 a $12 \mathrm{~mm}$, superior a $12 \mathrm{~mm}$ e Sem resposta;

- Diâmetro do Ventrículo Esquerdo na Diástole (DVED) (mm): categorizado em inferior a $60 \mathrm{~mm}$, de 60 a $75 \mathrm{~mm}$, superior a $75 \mathrm{~mm}$ e Sem resposta.

Embora existam rotinas automáticas para seleção de covariáveis, como backward, forward e stepwise, a seleção foi feita manualmente nos três cenários que consideram modelos multivariados (M1, M2 e M3). Inicialmente foi verificado o efeito de cada variável mais a FE contínua sobre a resposta, as variáveis que se apresentaram estatisticamente significativas 
foram retidas e ajustadas conjuntamente no modelo. Então, as variáveis foram removidas uma a uma de modo que se mantivesse no modelo a $\mathrm{FE}$, as outras três variáveis clinicamente importantes (IDADE, CLASSE e ETIO) e apenas outras variáveis estatisticamente significativas.

O modelo M1 foi avaliado no banco de dados com 2370 indivíduos (978 óbitos) e as variáveis significativas selecionadas foram: FE (na forma contínua), IDADE, CLASSE, ETIO, SEPTO, PAS, CREAT, ETIL, IMC, PAD, DVED, TRIGLI, SODIO e LEUC. Como citado, um grande problema da amostra é a presença de dados faltantes nas covariáveis. No caso do modelo M1, o problema foi contornado pela categorização das variáveis ao acrescentar uma categoria "sem resposta", como mencionado no Capítulo 2.

Mas, como discutido em Heller e Simonoff (1992), a categorização pré seleção de variáveis pode determinar se uma variável é significativa ou não dependendo da forma como ela for agrupada, além de afetar na significância de outras variáveis presentes no modelo (Maxwell e Delaney, 1993). Por isso, o ideal é avaliar se a variável contínua é relevante ao modelo para, então, discretizá-la se for necessário. Esta prática, apesar de indicada, pode não ser tão eficiente na presença de muitos dados faltantes e alguma forma para lidar com este problema deve ser encontrada. Uma possibilidade é usar técnicas de imputação de dados (Van Buuren et al. (1999) e White e Royston (2009)); o software estatístico $R$ disponibiliza os pacotes mice e mitools para imputações múltiplas. Por não ser a modelagem o foco do trabalho, a forma encontrada para lidar com este problema, usualmente aplicada (Clark e Altman, 2003), foi excluir as observações com dados faltantes para as variáveis clinicamente mais importantes: FE, IDADE, CLASSE e ETIO.

Ao remover os dados faltantes das principais covariáveis, a amostra em que os modelos M2 e M3 foram deduzidos ficou com 2083 indivíduos (878 óbitos). Neste contexto, as variáveis que melhor explicaram a resposta foram: FE (na forma contínua), IDADE, CLASSE, ETIO, SEPTO, PAS, CREAT, ETIL. Uma vez selecionadas as variáveis, o modelo M2 avalia a FE mantendo as variáveis contínuas durante a aplicação dos métodos e, portanto, espera-se perder menos informação ao determinar o melhor ponto de corte. Mas, por conta dos dados faltantes das outras variáveis selecionadas, também foi avaliado o modelo em que as variáveis significativas foram discretizadas após a seleção M3.

Alguns dos métodos descritos no decorrer do trabalho estão disponíveis em pacotes do software estatístico $R$. O gráfico do tempo de falha predito pelo modelo de Cox foi obtido pela função coxphQuantile do pacote clinfun. Para seleção do ponto de corte pelo método MPMIN3, o pacote maxstat disponibiliza a função maxstat.test que fornece, também, correção para o valor- $p$ pelos métodos Lausen92 e Lausen94. Neste mesmo pacote, as funções $p L a u s e n 92$ e $p L a u s e n 94$ fornecem o valor- $p$ corrigido para um dado valor- $p$ mínimo passado como parâmetro da função. Para seleção do ponto de corte pela estatística de teste corrigida segundo Contal e O'Quigley (1999) no cenário univariado, o pacote survMisc disponibiliza a função cutp. O restante dos métodos tiveram que ser programados. 


\subsection{Análise gráfica}

Para selecionar bons pontos de corte para uma variável explicativa contínua, primeiramente, deve-se avaliar graficamente a relação desta variável com a resposta. Três gráficos foram propostos no Capítulo 3, na Seção 3.2. Os gráficos referentes aos resíduos martingal e ao tempo de falha predito são aplicáveis em todos os cenários, já o gráfico do tempo de falha predito segundo método não-paramétrico de Kaplan-Meier (KM) é válido somente para o cenário univariado. .

Por conta da alta proporção de censuras (aproximadamente 60\%), ao separar a variável em grupos definidos pelos decis da FE, em alguns grupos não foi possível estimar o tempo de falha $t$ pelo estimador de Kaplan-Meier, de modo que $S(t \mid \mathrm{FE}) \leq 0.5$. Portanto, em vez de usar o tempo de falha mediano, o tempo em que $60 \%$ das observações permanecem livres de falha foi usado para predição no cenário univariado. O mesmo argumento é utilizado para justificar a apresentação de dois gráficos de tempo de falha predito por meio do modelo de regressão de Cox.

Supondo um modelo com as variáveis explicativas representadas por $\boldsymbol{x}^{\prime}=(\mathrm{FE}, \boldsymbol{z})$, em que $\boldsymbol{z}$ é o vetor do restante das covariáveis associadas ao tempo de falha presentes no modelo, o gráfico que plota o tempo de falha predito 1 versus a fração de ejeção apresenta os tempos preditos por

$$
\hat{S}_{0}(\hat{t})=(0.5)^{\exp \left(-\boldsymbol{x}^{\prime} \hat{\boldsymbol{\beta}}\right)}
$$

em que $50 \%$ das observações permanece em risco. O gráfico que plota o tempo de falha predito 2 versus a fração de ejeção apresenta os tempos preditos por

$$
\hat{S}_{0}(\hat{t})=(0.6)^{\exp \left(-\boldsymbol{x}^{\prime} \hat{\boldsymbol{\beta}}\right)}
$$

em que $60 \%$ das observações permanece livre de falha e $\hat{\boldsymbol{\beta}}$ é vetor de parâmetros estimados associados a $\boldsymbol{x}^{\prime}$. Para construção dos gráficos dos tempos de falha preditos via modelo de Cox, foram considerados os valores médios das demais covariáveis presentes no modelo de Cox $(\boldsymbol{z})$. A função do $R$ usada para gerar tais gráficos exige a construção do gráfico de dispersão dos tempos de falha observados versus a variável de principal interesse, e permite assumir outros valores para o restante das variáveis, que não o valor médio.

A Figura 5.1 apresentada a seguir é subdividida em três figuras menores, 5.1(a), 5.1(b) e 5.1(c), referentes aos cenários M1, M2, M3, respectivamente. Cada uma delas apresenta três gráficos: resíduos martingal versus $\mathrm{FE}$, tempo de falha predito 1versus $\mathrm{FE}$ e tempo de falha predito 2 versus FE. A Figura 5.2, referente ao cenário Uni, apresenta 4 gráficos: resíduos martingal versus $\mathrm{FE}$, tempo de falha predito via Kaplan-Meier (KM) versus FE e os dois inferiores apresentam os tempos de falha preditos 1 e 2 via modelo de regressão de Cox versus FE.

Os gráficos dos resíduos martingal e dos tempos de falha preditos apresentam comportamentos parecidos entre os cenários. A curva suavizada dos gráficos dos resíduos fica próxima 
de zero e apresenta mudança leve no comportamento entre os valores 30 e 40 da FE, portanto, a categorização da variável pode ser dispensada, caso seja possível; caso contrário, deve-se procurar por um valor entre 30 e 40 para categorização.

Os gráficos dos tempos de falha preditos pelo modelo de Cox apresentam uma relação monótona e, portanto, a dicotomia e a tricotomia podem ser aplicadas. Os gráficos dos tempos de falha preditos 1 têm mudança na inclinação da curva mais aparentes entre os pontos 30 e 40, enquanto que os gráficos relativos aos tempos de falha preditos 2 têm mudança mais aparente entre os pontos 50 e 60. O gráfico sobre o tempo de falha predito por KM indica picos nos pontos 30,40 e 60 aproximadamente.

Por sugestão gráfica, todos os métodos de seleção de ponto de corte, validação e correção dos resultados, nos casos de dicotomia e tricotomia, devem considerar como potenciais pontes de corte os valores da FE contidos no intervalo aproximado [30,60], que é bem representado ao tomar os $60 \%$ valores centrais da variável contínua $\mathrm{FE}$, ou seja, tomar $\epsilon=0.2$ como a proporção dos valores que devem ser desconsiderados na busca. Assim, para os cenários M1 e Uni, a busca pelo ponto de corte foi feita no intervalo [25, 60] e para os cenários M2 e M3, no intervalo $[25,58]$. A leve mudança nos intervalos de possíveis pontos de corte é resultado da alteração das amostras nos diferentes cenários devido ao problema de dados faltantes.

\subsection{Resultados para dicotomia}

Para o caso de dicotomia, procura-se um ponto de corte $c$ que separe os pacientes segundo o grupo com FE reduzida $(\mathrm{FE} \leq c)$ e o grupo com $\mathrm{FE}$ preservada $(\mathrm{FE}>c)$. A Tabela 5.1 apresenta os pontos de corte e riscos relativos estimados por MDV (MDV1 e MDV2) e a Tabela 5.2 apresenta os pontos de corte, riscos relativos e valores- $p$ estimados por MPMIN (MPMIN1, MPMIN2 e MPMIN3), em que o risco relativo representa a taxa de óbito entre os pacientes com FE preservada em relação aos pacientes com FE reduzida.

Tabela 5.1: Ponto de corte $c$ e risco relativo estimados via MDV nos 4 cenários.

\begin{tabular}{cccc} 
Cenário & Método & $\boldsymbol{c}$ & risco relativo \\
\hline \multirow{2}{*}{ M1 } & MDV1 & 40 & 0.7991 \\
& MDV2 & 26 & 0.7913 \\
M2 & MDV1 & 34 & 0.6145 \\
& MDV2 & 33 & 0.6072 \\
M3 & MDV1 & 32 & 0.6118 \\
& MDV2 & 27 & 0.5925 \\
\multirow{2}{*}{ Uni } & MDV1 & 27 & 0.5720 \\
& MDV2 & 28 & 0.5686 \\
\hline
\end{tabular}

Comparação entre os cenários: Para MDV, o cenário Uni apresenta valores baixos de $c$ e o cenário M1 apresenta o maior valor e o menor. Já MPMIN não sofre grande alteração com a mudança de cenário. Quanto aos riscos relativos, o cenário M1 apresenta as maiores estimativas para ambos os métodos $(\geq 0.73)$, enquanto que, para os outros cenários, as 


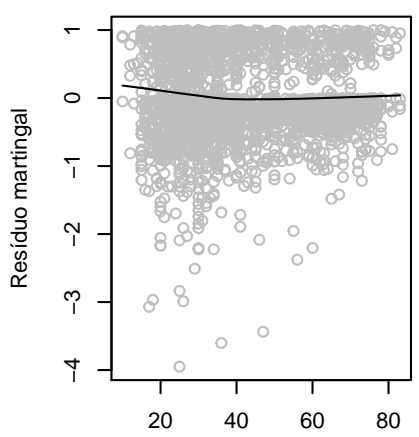

Fração de ejeção (\%)

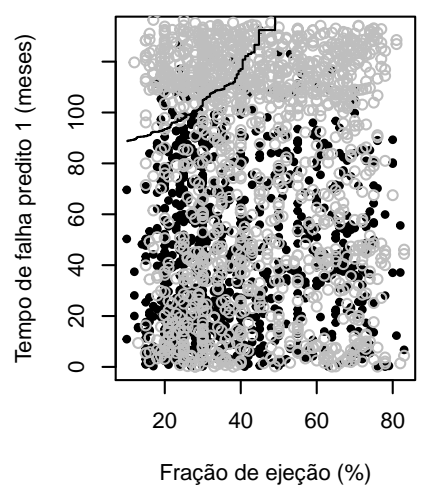

Fração de ejeção (\%)

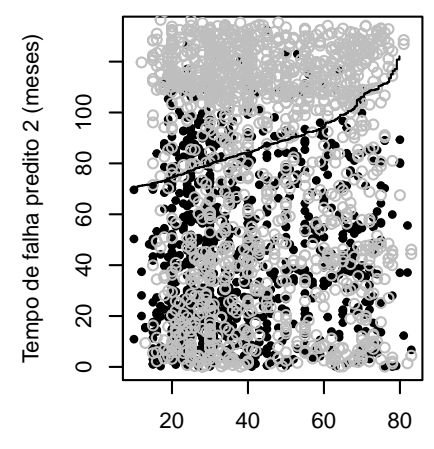

Fração de ejeção (\%)

(a) Figura 1 - referente ao cenário M1
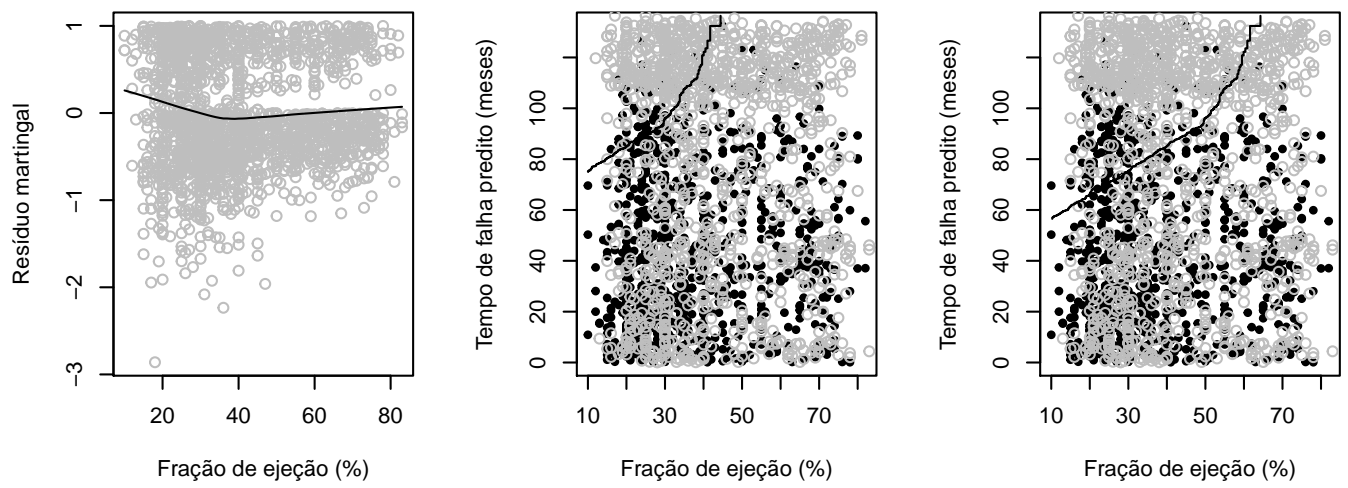

(b) Figura 2 - referente ao cenário M2
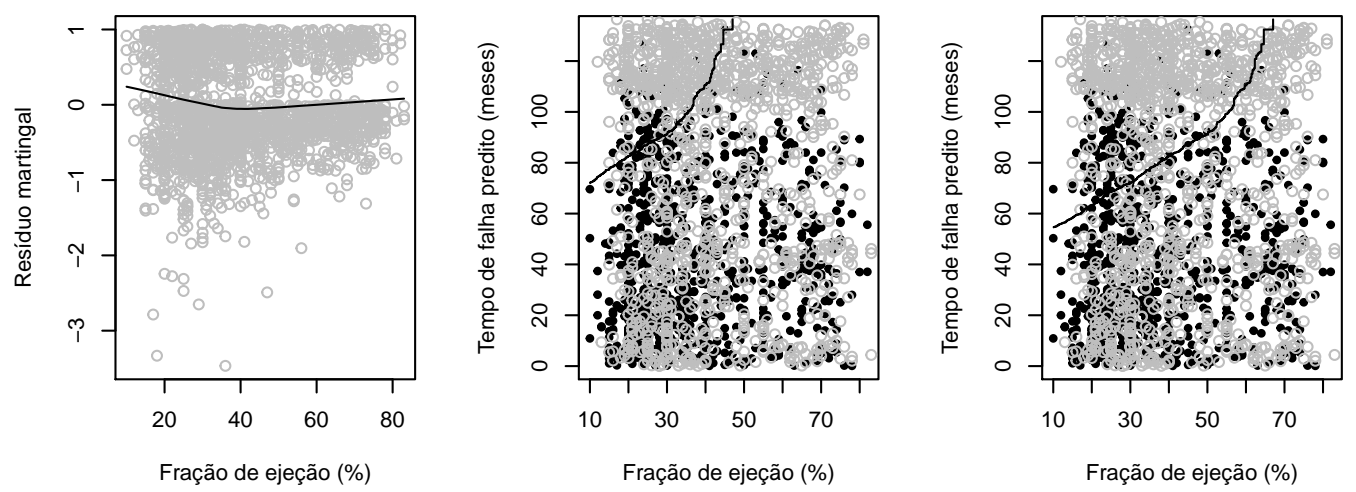

(c) Figura 3 - referente ao cenário M3

Figura 5.1: As figuras 1,2 e 3 apresentam os mesmos três gráficos em cada um delas. Gráfico 1 plota FE $\times$ Resíduo Martingal; Gráficos 2 e 3 apresentam os tempos de falha preditos versus $F E$, em que o tempo foi predito pelo modelo de Cox de tal modo que $S(t / F E)=0.5$ e $S(t / F E)=0.6$, respectivamente. Gráficos 2 e 3 são plotados sobre o gráfico de dispersão da FE pelos tempos de falha observados, em que os pontos pretos são os óbitos e os pontos cinzas são as censuras. 


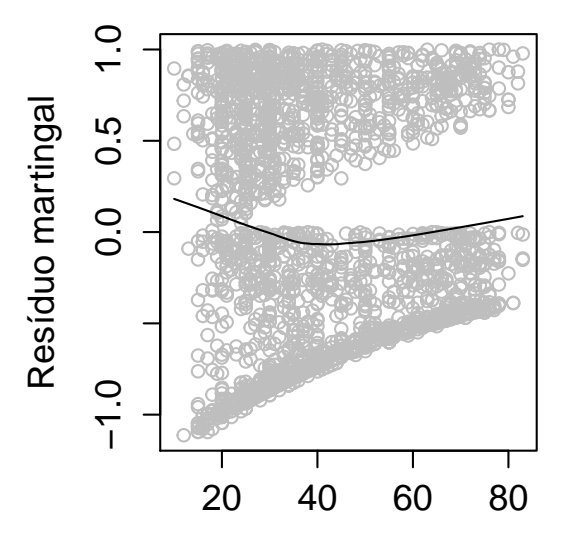

Fração de ejeção (\%)
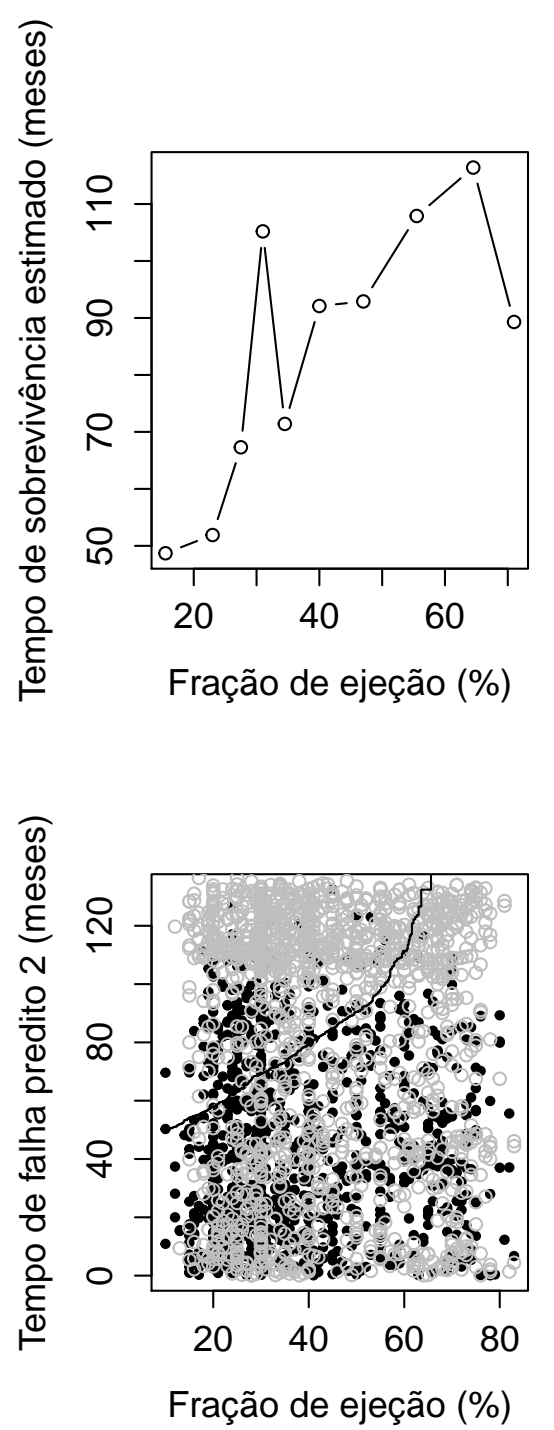

Figura 5.2: Os gráficos foram construídos com base no modelo de Cox ajustado para o cenário Uni. Gráfico 1 plota FE×Resíduo Martingal; Gráficos 2 plota o tempo de sobrevivência estimado pelo método de Kaplan-Meier versus o ponto médio dos grupos definidos pelos decis da variável FE e os Gráficos 3 e 4 apresentam os tempos de falha preditos 1 e 2 versus $F E$, em que o tempo foi estimado pelo modelo de Cox de tal modo que $S(t / F E)=0.5$ e $S(t / F E)=0.6$, respectivamente. Gráficos 3 e 4 são plotados sobre o gráfico de dispersão da FE pelos tempos de falha observados, em que os pontos pretos são os óbitos e os pontos cinzas são as censuras.

estimativas são de aproximadamente 0.6.

Comparação entre os métodos: MDV apresenta maior variabilidade entre os pontos de corte estimados entre os cenários (26 a 40) ao contrário de MPMIN (29 e 30). Ambos os métodos sugerem riscos relativos muito próximos.

Mazumdar e Glassman (2000) sugeriu avaliar os riscos relativos para auxiliar no processo de escolha do ponto de corte, pois as estatísticas de teste tendem a ser muito sensíveis ao tamanho da amostra. Segundo o critério do risco relativo, o ideal é selecionar aquele que apresenta a maior diferença entre os grupos avaliados. Os menores riscos (aproximadamente $0.57)$ foram estimados nos cenários: Uni com o método MDV2 $(c=28)$ e M2 com os métodos 
Tabela 5.2: Ponto de corte c, risco relativo e valor-p estimados via MPMIN nos 4 cenários.

\begin{tabular}{ccccc} 
Cenário & Método & $\boldsymbol{c}$ & risco relativo & valor- $\boldsymbol{p}$ \\
\hline \multirow{2}{*}{ M1 } & MPMIN1 & 29 & 0.7302 & 0.0001 \\
& MPMIN2 & 29 & 0.7302 & 0.0001 \\
M2 & MPMIN1 & 29 & 0.5686 & $<0.0001$ \\
& MPMIN2 & 29 & 0.5686 & $<0.0001$ \\
M3 & MPMIN1 & 30 & 0.5888 & $<0.0001$ \\
& MPMIN2 & 30 & 0.5888 & $<0.0001$ \\
\multirow{2}{*}{ Uni } & MPMIN1 & 30 & 0.5743 & $<0.0001$ \\
& MPMIN2 & 30 & 0.5743 & $<0.0001$ \\
& MPMIN3 & 30 & 0.5743 & $<0.0001$ \\
\hline
\end{tabular}

MPMIN1 e MPMIN2 $(c=29)$.

Tabela 5.3: Resultados das estatísticas de teste corrigidas pelos métodos de Contal O'Quigley e Jespersen.

\begin{tabular}{cccc} 
Cenário & Método & $\boldsymbol{c}$ & valor- $\boldsymbol{p}$ \\
\hline M1 & Correção de Contal e O'Quigley Multivariado & 42 & 0.3206 \\
M2 & Correção de Contal e O'Quigley Multivariado & 40 & 0.4865 \\
M3 & Correção de Contal e O'Quigley Multivariado & 40 & 0.5404 \\
\multirow{2}{*}{ Uni } & Correção de Contal e O'Quigley Univariado & 31 & $<0.0001$ \\
& Correção de Jespersen & 30 & $<0.0001$ \\
\hline
\end{tabular}

A Tabela 5.3 apresenta os pontos de corte e valores- $p$ obtidos pelas estatísticas corrigidas de Contal e O’Quigley (1999) e de Jespersen. A estatística de Jespersen é baseada na padronização da estatística de teste score e, portanto, fornece o mesmo ponto de corte selecionado por MPMIN3. Para o cálculo dos valores- $p$ associados à correção de Contal e O'Quigley nos cenários multivariados foram utilizadas dez iterações na expressão da distribuição assintótica das estatísticas de teste dada por (3.8). As correções para o valor-p dos cenários multivariados são baseados nos resíduos de Cox-Snell dos modelos, como resíduos com valores altos foram observados, justifica-se os valores- $p$ corrigidos muito acima dos valores desejados $(\leq 0.05)$. Portanto, para os cenários multivariados há indícios de que a dicotomia da variável FE não é indicada, o contrário pode ser observado para o cenário univariado para ambos os métodos.

\subsubsection{Correção dos resultados}

As tabelas C.3 e C.4, disponíveis no Apêndice C, apresentam os resultados de correção dos riscos relativos estimados por MDV e riscos relativos e valores- $p$ estimados por MPMIN, respectivamente. Os resultados foram obtidos a partir de 1000 repetições do método de validação cruzada dupla. Para cada iteração, os dois métodos de seleção de pontos de corte eram aplicados e os valores dos riscos relativos e valores- $p$ foram retidos. As tabelas apresentam as estatísticas média, média aparada (avaliando apenas os $80 \%$ valores centrais), moda, mediana e desvio padrão relacionadas aos valores retidos a partir das 1000 reamostragens. 
A moda é a estatística usada para definir o valor corrigido.

Para os métodos MDV e MPMIN, os cenários M2, M3 e Uni apresentam resultados similares para os riscos relativos, aproximadamente 0.60 , com desvio padrão aproximado de 0.02 , já o cenário M1 apresenta valores mais altos para ambos os métodos (0.81), acompanhados de desvios maiores (0.04). Quanto aos valores- $p$, a única combinação de método e cenário que apresentou alguma das estatísticas superior a 0.01 foi o método MPMIN2 no cenário M1, ainda assim, a moda se apresentou igual a 0.01, com desvio de 0.14.

A aplicação dos métodos Lausen92 e Lausen94 para correção do valor- $p$ resultou em valores inferiores a 0.01 em todos os cenários para os três testes, MPMIN1, MPMIN2 e MPMIN3; o valor de $\epsilon$ considerado foi igual 0.2. E os riscos relativos corrigidos pelos fatores de contração heurístico, boostrap e validação cruzada, disponíveis no C na Tabela C.9, resultaram valores próximos a 0.6 ; note que os valores de $\hat{d}$ foram próximos a 1 , o que indica que o risco relativo foi pouco superestimado pelo modelo categorizado. O fator de contração vc foi deduzido pela média de 100 fatores. O fator de contração adhoc não foi implementado, pois os valores- $p$ estimados por MPMIN foram muito próximos a zero.

\subsection{Resultados para tricotomia}

Para o caso de tricotomia, procura-se o vetor de pontos de corte $c^{\prime}=(c 1, c 2)$ que separa os pacientes segundo o grupo com FE reduzida $(\mathrm{FE} \leq c 1)$, FE limítrofe $(c 1<F E \leq c 2)$ e o grupo com FE preservada $(\mathrm{FE}>c 2)$. A Tabela 5.4 apresenta os pontos de corte e os riscos relativos 1 e 2 estimados por MDV (MDV1 e MDV2) e a Tabela 5.5 apresenta os pontos de corte, riscos relativos 1 e 2 e valores- $p$ estimados por MPMIN (MPMIN1, MPMIN2 e MPMIN3), em que o risco relativo 1 ( $r r 1$ ) representa a taxa de óbito entre os pacientes com FE limítrofe em relação aos pacientes com FE reduzida e o risco relativo $2(r r 2)$ representa a taxa de óbito entre os pacientes com FE preservada em relação aos pacientes com FE reduzida.

Comparação entre os cenários: Os valores de c1 são mais altos no cenário M1 e são parecidos no restante dos cenários, para ambos os métodos. Os valores de $c 2$ variam bastante entre os cenários para MDV (de 45 a 57) e, para MPMIN, são próximos apenas nos cenários M2, M3 e Uni. Em relação aos riscos relativos, é importante avaliar a distância $r r 1$ - rr2, pois espera-se que o risco relativo 1 seja maior do que o risco relativo 2, por motivos clínicos. Os riscos 1 e 2 foram parecidos entre os cenários M2 e M3, dentro de cada método. No cenário M1, os riscos 1 e 2 são mais próximos e no cenário UNI, são mais distantes.

Comparação entre os métodos: A distância entre os pontos de corte estimados $(c 2-c 1)$ variaram de 5 a 25 para o método MDV entre os cenários, ao contrário de MPMIN, que apresentou pontos de corte parecidos e a distância foi de aproximadamente 5 (exceto em M1 que apresentou 11). Para MDV, a menor distância entre os pontos de corte foi verificada no cenário M1 e a maior no cenário UNI. Para MDV, o cenário M1 apresentou $r r 2>r r 1$, indicando que o ajuste com os pontos de corte selecionados por este método não forneceu um resultado 
clinicamente significativo. O melhor modelo, no sentido de riscos relativos mais distantes, foi ajustado no cenário UNI com pontos de corte estimados por MPMIN ( $c 1=25$ e $c 2=30)$.

Tabela 5.4: Vetor de pontos de corte $c^{\prime}=(c 1, c 2)$, riscos relativos 1 e 2 estimados via $M D V$ nos 4 cenários.

\begin{tabular}{cccccc} 
Cenário & Método & $\boldsymbol{c 1}$ & $\boldsymbol{c 2}$ & risco relativo 1 & risco relativo 2 \\
\hline \multirow{2}{*}{ M1 } & MDV1 & 40 & 45 & 0.7338 & 0.8202 \\
& MDV2 & 35 & 54 & 0.7816 & 0.7828 \\
M2 & MDV1 & 34 & 56 & 0.6306 & 0.5830 \\
& MDV2 & 34 & 51 & 0.6398 & 0.6051 \\
M3 & MDV1 & 33 & 52 & 0.6398 & 0.5844 \\
& MDV2 & 33 & 53 & 0.6364 & 0.5795 \\
\multirow{2}{*}{ Uni } & MDV1 & 32 & 46 & 0.6704 & 0.5609 \\
& MDV2 & 26 & 52 & 0.6306 & 0.4974 \\
\hline
\end{tabular}

Tabela 5.5: Vetor de pontos de corte $c^{\prime}=(c 1, c 2)$, riscos relativos 1 e 2 e valor-p estimados via MPMIN nos 4 cenários.

\begin{tabular}{ccccccc} 
Cenário & Método & $\boldsymbol{c 1}$ & $\boldsymbol{c 2}$ & risco relativo 1 & risco relativo 2 & Valor- $\boldsymbol{p}$ \\
\hline \multirow{2}{*}{ M1 } & MPMIN1 & 29 & 40 & 0.7598 & 0.6694 & 0.0003 \\
& MPMIN2 & 29 & 40 & 0.7598 & 0.6694 & 0.0003 \\
M2 & MPMIN1 & 25 & 30 & 0.7278 & 0.5059 & $<0.0001$ \\
& MPMIN2 & 25 & 30 & 0.7278 & 0.5059 & $<0.0001$ \\
M3 & MPMIN1 & 25 & 31 & 0.7590 & 0.5201 & $<0.0001$ \\
& MPMIN2 & 25 & 31 & 0.7590 & 0.5201 & $<0.0001$ \\
\multirow{2}{*}{ Uni } & MPMIN1 & 25 & 30 & 0.7809 & 0.5202 & $<0.0001$ \\
& MPMIN2 & 25 & 30 & 0.7809 & 0.5202 & $<0.0001$ \\
& MPMIN3 & 25 & 30 & 0.7809 & 0.5202 & $<0.0001$ \\
\hline
\end{tabular}

\subsubsection{Correção dos resultados}

As tabelas C.7 e C.8, disponíveis no Apêndice C, apresentam os resultados para correção dos riscos relativos estimados por MDV e riscos relativos e valores- $p$ estimados por MPMIN, respectivamente. Os resultados foram obtidos por meio de 1000 repetições do método de validação cruzada. Para cada iteração, os dois métodos de seleção de pontos de corte foram aplicados, MDV e MPMIN, e os valores dos riscos relativos, $r r 1$ e $r r 2$, e valores- $p$ foram retidos. As tabelas apresentam as estatísticas média, média aparada, moda, mediana e desvio padrão relacionadas aos valores retidos a partir das 1000 reamostragens.

Os métodos MDV1 e MDV2 apresentam respostas diferentes entre si e se comportam de forma parecida entre os cenários M2, M3 e Uni. Nestes cenários, MDV1 indica rr1 aproximadamente igual a 0.65 e $r r 2$ aproximadamente igual a 0.60 , enquanto MDV2 indica $r r 1$ aproximadamente igual 0.63 e $r r 2$ aproximadamente 0.61 (desvios próximos a 0.04 ). No cenário M1, os métodos MDV1 e MDV2 se comportam de forma parecida e apresentam 
valores mais altos para os riscos estimados, sendo $r r 1$ aproximadamente igual a 0.80 e $r r 2$ aproximadamente igual a 0.77 (desvios próximos a 0.06).

Não é possível identificar um padrão nas respostas obtidas pelos métodos MPMIN1, MPMIN2 e MPMIN3. Entretanto, observa-se que, para os cenário M2, M3 e Uni, os valores de $r r 1$ se concentram próximos a 0.65 e os valores de $r r 2$ se concentram próximos a 0.55 (desvio padrão de aproximadamente 0.03); além disso, como no método MDV, observa-se valores mais altos no cenário M1, aproximadamente 0.79 para $r r 1$ e 0.77 para $\operatorname{rr} 2$ (desvio de aproximadamente 0.05). Ademais aos riscos mais altos, para o cenário M1, as estatísticas para os valores- $p$ foram superiores ou iguais a 0.01, mas ainda assim, a correção indica nível descritivo menor ou igual a 0.05 . Note que as estimativas para $r r 2$ foram inferiores às estimativas feitas por MDV e a distância entre os riscos foram superiores às distâncias dos riscos estimados por MDV.

\subsection{Validação dos pontos de corte, valores- $p$ e riscos relativos}

A fim de identificar estimativas de pontos de corte e de riscos relativos robustas à variabilidade dos dados, o método de reamostragem bootstrap foi aplicado aos dados. Foram feitas 2000 reamostragens para cada combinação de método de seleção de ponto de corte (MDV e MPMIN), cenário (M1, M2, M3 e Uni) e quantidade de grupos (dicotomia e tricotomia), e foram extraídos os respectivos valores dos pontos de corte, valores- $p$ e riscos relativos estimados a partir do modelo categorizado. O tamanho de cada amostra bootstrap foi igual ao tamanho da amostra considerada em cada cenário. Dentro de cada combinação, a moda dos pontos de corte, a moda dos valores- $p$ e a moda dos riscos relativos definirão os valores validados.

Para avaliar o comportamento das amostras bootstrap, no Apêndice C estão disponíveis as tabelas com as estatísticas média, média aparada, moda, mediana e desvio padrão para os valores estimadas para cada combinação e no Apêndice D estão disponíveis os histogramas da distribuição dos pontos de corte e riscos relativos estimados. Para dicotomia, as tabelas C.1 e C.2 e as figuras D.1 e D.2 estão relacionadas às amostras bootstrap calculadas sobre os métodos MDV e MPMIN, respectivamente. Para o caso de tricotomia, a Tabela C.5 e as figuras D.3 e D.4 estão relacionadas às amostras bootstrap calculadas sobre os métodos MDV1 e MDV2, e a Tabela C.6 e as figuras D.5, D.6 e D.7 estão relacionadas às amostras bootstrap calculadas sobre os métodos MPMIN1, MPMIN2 e MPMIN3. Todos os valores$p$ estimados nas amostras bootstrap em todas as combinações foram inferiores a 0.01 e, portanto, os resultados foram omitidos das tabelas a seguir.

Ao avaliar a Tabela 5.6 e as outras tabelas e figuras disponíveis nos apêndices sobre os valores obtidos nas amostras bootstrap para dicotomia, observa-se que os valores de ponto de corte $(c)$ estimados por MDV se concentram entre os valores de 30 a 35 e os valores estimados por MPMIN se concentram no valor 30, aproximadamente. Os histogramas dos riscos relativos $(r r)$ se assemelham à curva da distribuição Normal; para MDV as curvas são 
Tabela 5.6: Moda dos 2000 pontos de corte e moda dos 2000 riscos relativos estimados pelos métodos MDV e MPMIN nas amostras bootstrap, para os cenários M1, M2, M3 e Uni, no caso de dicotomia.

\begin{tabular}{ccccccc} 
& & \multicolumn{5}{c}{ Método } \\
\cline { 3 - 7 } Medida & Cenário & MDV1 & MDV2 & MPMIN1 & MPMIN2 & MPMIN3 \\
\hline \multirow{4}{*}{ c } & M1 & 40 & 35 & 29 & 29 & - \\
& M2 & 36 & 34 & 29 & 29 & - \\
& M3 & 35 & 31 & 29 & 29 & - \\
& Uni & 35 & 31 & 30 & 30 & 30 \\
& M1 & 0.78 & 0.76 & 0.69 & 0.69 & - \\
rr & M2 & 0.60 & 0.59 & 0.55 & 0.55 & - \\
& M3 & 0.61 & 0.58 & 0.57 & 0.57 & - \\
& Uni & 0.59 & 0.60 & 0.55 ou 0.57 & 0.57 & 0.57 \\
\hline
\end{tabular}

aproximadamente centradas em 0.60 (exceto para o cenário M1, que é centrado em 0.75 , aproximadamente) e para MPMIN, são aproximadamente centradas em 0.55 (exceto para o cenário M1, que é centrado em 0.70, aproximadamente).

Para ambos os métodos, a variabilidade da resposta, medida pelo desvio padrão, foi maior para o cenário M1. Para MDV, o desvio para os pontos de corte no cenário M1 é de aproximadamente 8.0 e para o restante dos cenários é de aproximadamente 4. Para MPMIN, o desvio para os pontos no cenário M1 é de aproximadamente 4.9 e para o resto dos cenários é aproximadamente 2.6. Em relação a variabilidade dos riscos, para os método MDV e MPMIN no cenário M1, os desvios foram de aproximadamente 0.09 e 0.06, respectivamente, no restante dos cenários o desvio foi de aproximadamente 0.04 para os dois métodos. O cenário M1 apresentou estimativas para o risco relativo superiores a 1 para os dois métodos.

Em relação aos pontos de corte estimados sem ajustes, disponíveis nas tabelas 5.1 e 5.2, apesar do cenário M1 apresentar valores mais altos para os pontos validados, ao avaliar os histogramas, é possível verificar que o intervalo em que valores de $c$ se concentram no cenário M1 também abrange o intervalo em que os valores de $c$ se concentram nos demais cenários.

Em relação às estimativas de $c$ e $r r$, MDV apresenta menor variabilidade nas estimativas validadas entre os cenários e MPMIN manteve a característica de estimativas robustas à troca de cenário e de teste escolhido (MPMIN1, MPMIN2 ou MPMIN3). MDV2 tende a ter resultados mais parecidos ao MPMIN do que ao MDV1. Ambos os métodos, com ou sem validação apresentaram riscos relativos aproximadamente iguais a 0.6 (exceto o cenário M1), mas para os resultados validados, os riscos relativos estimados por MPMIN, sugerem uma leve diminuição para 0.55 .

Ao avaliar o qualidade dos pontos de corte estimados segundo o critério do risco relativo, os pontos de corte selecionados são: $c=29$ estimado por MPMIN1 e MPMIN2 no cenário M2, e $c=30$ estimado por MPMIN1 no cenário Uni, o risco relativo estimado foi de 0.55.

Ao avaliar a Tabela 5.7 e as outras tabelas e figuras disponíveis nos apêndices sobre os valores obtidos nas amostras bootstrap para tricotomia, observa-se que os valores estimados do ponto de corte $c 1$ se concentram próximos a 34 para MDV e próximos a 25 para MPMIN, 
Tabela 5.7: Moda dos 2000 pontos de corte e 2000 riscos relativos estimados pelos métodos MDV e MPMIN nas amostras bootstrap, para os cenários M1, M2, M3 e Uni, no caso de tricotomia.

\begin{tabular}{ccccccc}
\multirow{2}{*}{ Medida } & Cenário & MDV1 & MDV2 & MPMIN1 & MPMIN2 & MPMIN3 \\
\cline { 3 - 7 } & M1 & 34 & 26 & 29 & 29 & - \\
c1 & M2 & 36 & 34 & 25 & 25 & - \\
& M3 & 34 & 26 & 25 & 25 & - \\
& Uni & 32 & 32 & 25 & 25 & 25 \\
& M1 & 0.8 & 0.77 & 0.76 & 0.76 & - \\
rr1 & M2 & 0.63 & 0.62 & 0.67 & 0.63 & - \\
& M3 & 0.63 & 0.63 & 0.65 & 0.65 & - \\
& Uni & 0.66 & 0.64 & 0.66 & 0.70 & 0.70 \\
& M1 & 57 & 54 & 40 & 40 & - \\
c2 & M2 & 45 & 45 & 30 & 30 & - \\
& M3 & 54 & 54 & 30 & 35 & - \\
& Uni & 55 & 45 ou 56 & 40 & 40 & 40 \\
& M1 & 0.74 & 0.74 & 0.57 ou 0.62 & 0.57 & - \\
rr2 & M2 & 0.55 & 0.56 & 0.46 & 0.46 & - \\
& M3 & 0.55 & 0.55 ou 0.57 & 0.48 & 0.48 & - \\
& Uni & 0.61 & 0.61 & 0.47 & 0.48 & 0.48 \\
\hline
\end{tabular}

enquanto que, para $c 2$, os histogramas se concentram nos pontos 45 e 54 para MDV e entre os pontos 30 e 40 para MPMIN. Note que valores próximos a 30 aparecem como estimativa para $c 1$ ou para $c 2$, o que indica que tal valor deve ser, de fato, um ponto de mudança de comportamento na resposta dos pacientes.

Para MDV e MPMIN, os valores validados de $c 1$ se concentram no mesmo intervalo das estimativas não ajustadas, e o mesmo pode ser observado para $c 2$ validados. Quanto aos riscos relativos, ao avaliar os histogramas que apresentam uma silhueta similar à curva da distribuição Normal, as estimativas de $r r 1$ para ambos os métodos, independente do cenário, são aproximadamente iguais a 0.64 , já os valores de $r r 2$ se concentram em 0.56 para MDV e em 0.47 para MPMIN. O método MPMIN sugere maior diferença entre os riscos dos grupos gerados pelos pontos de corte $c 1$ e $c 2$.

Os valores estimados e validados para $r r 1$ e $r r 2$ segundo MDV são muito parecidos, por outro lado, para MPMIN, apenas os valores validados de $r r 2$ são similares, a validação para rr1 indica uma redução aproximada de 0.1 . Note que, após a validação dos dados, a diferença de riscos entre os grupos gerados pelos pontos de corte foi reduzida para MPMIN. Ao avaliar o qualidade dos pontos de corte estimados segundo o critério do risco relativo, os pontos de corte selecionados são: $c 1=25$ e $c 2=40$ estimados por MPMIN2 e MPMIN3 no cenário Uni, os riscos relativos estimados foram de $r r 1=0.70$ e $r r 2=0.48$. 


\subsection{Comparação dos métodos}

A fim de comparar os métodos e cenários avaliados anteriormente, uma configuração única de amostra e variáveis significativas ao modelo foi definida para reaplicação dos métodos de seleção de pontos de corte e correção dos valores estimados. Por serem importantes sob o ponto de vista clínico, as variáveis IDADE, CLASSE e ETIO serão usadas como únicas variáveis no ajuste dos modelos M1, M2, além da FE. Também sob o ponto de vista estatístico, as três variáveis são significativas ao modelo independente do cenário. Entre elas, apenas a variável IDADE é contínua e será, portanto, categorizada no cenário M1. Obviamente, o cenário univariado não sofrerá alteração em relação ao ajuste do modelo e o cenário M3 passa a ser equivalente ao cenário M1. Quanto ao tamanho da amostra, as observações com dados faltantes para alguma das três variáveis foram removidas da amostra, restando 2083 observações (878 óbitos).

\subsubsection{Análise gráfica}

Os gráficos apresentados nesta seção são os mesmos apresentados na Seção 5.1. A Figura 5.3 apresenta as figuras 5.3(a) e 5.3(b) referentes aos cenários M1 e M2, respectivamente. Cada uma das figuras apresenta três gráficos: resíduos martingal versus FE, tempo de falha predito 1 versus FE e tempo de falha predito 2 versus FE. A Figura 5.4, referente ao cenário Uni, apresenta 4 gráficos: resíduos martingal versus FE, tempo de falha predito via KaplanMeier (KM) versus $\mathrm{FE}$ e os dois inferiores apresentam os tempos de falha 1 e 2 preditos via modelo de regressão de Cox versus FE.

Ao analisar os três tipos de gráficos apresentados na Figura 5.3, as conclusões entre os cenários foram similares e, também, foram parecidas com os resultados encontrados na Seção 5.1. Os gráficos dos resíduos martingal por apresentarem mudança leve no comportamento da curva entre os valores 30 e 40 indicam que a categorização da variável pode ser dispensada, caso seja possível. Os gráficos que representam a relação entre o tempo de falha predito e a FE apresentam uma relação monótona e, portanto, a dicotomia e a tricotomia podem ser avaliadas. Mudanças mais aparentes na inclinação das curvas foram verificadas entre os pontos $[30,40]$ e $[50,60]$. Ao avaliar a Figura 5.4, têm-se conclusões parecidas entre os mesmos gráficos em relação a (5.3), já para o gráfico do tempo de falha predito por Kaplan-Meier, observam-se picos próximos aos valores 40 e 55 da FE.

Sem evidências claras dos pontos de corte a serem utilizados, a busca pelos pontos deve ser feita no intervalo [30,60]. Tal intervalo é bem representado ao tomar os $60 \%$ valores centrais da variável contínua FE como potenciais pontos de corte e, então, todos os métodos de seleção de ponto de corte, validação e correção dos resultados, para dicotomia e tricotomia, consideraram o intervalo $[25,58]$. 


\subsubsection{Resultados para dicotomia}

A Tabela 5.8 apresenta os pontos de corte e riscos relativos estimados pelo MDV (MDV1 e MDV2) e a Tabela 5.9 apresenta os pontos de corte, riscos relativos e valores- $p$ estimados pelo MPMIN (MPMIN1, MPMIN2 e MPMIN3). Quanto aos pontos de corte, o método MDV1 apresentou aproximadamente o mesmo valor para $c$ (33), independentemente do cenário; o mesmo pode ser observado para MDV2 que estimou como ponto de corte ideal o valor 26. As estimativas pelo método MPMIN apresentam pouca variabilidade $(c=28$ ou $c=29)$. As estimativas para MDV2 se apresentam mais próximas das estimativas para MPMIN.

Quanto aos riscos relativos, os menores valores são observados para os métodos MDV2 e MPMIN $(<0.6)$, a menor estimativa para o risco é obtida pelo método MPMIN no cenário Uni com valor aproximado de 0,58 e ponto de corte $c=28$.
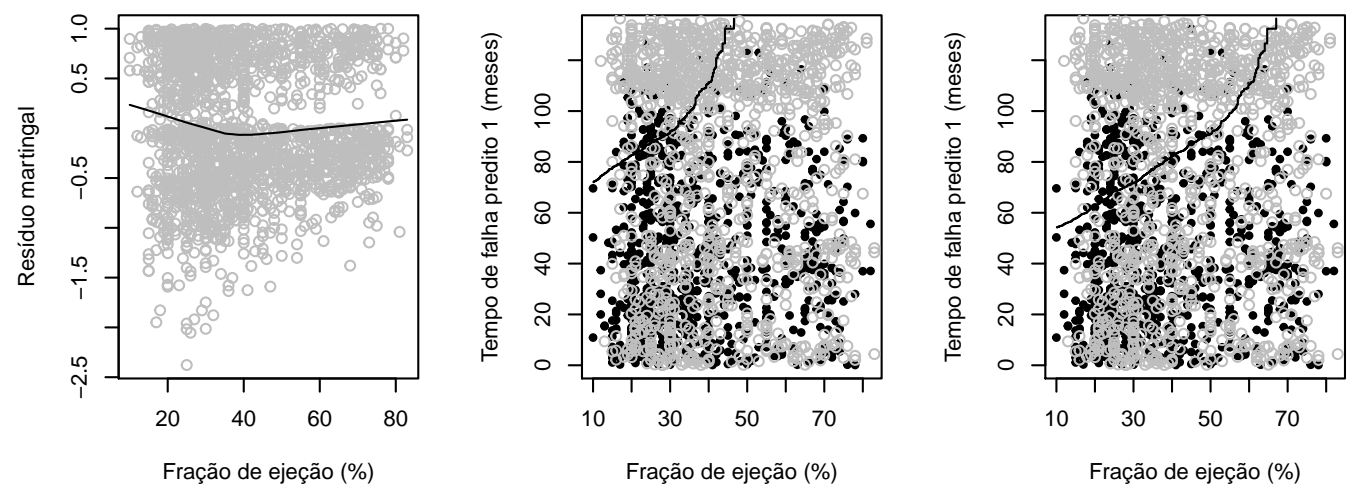

(a) Figura 1 - referente ao cenário M1
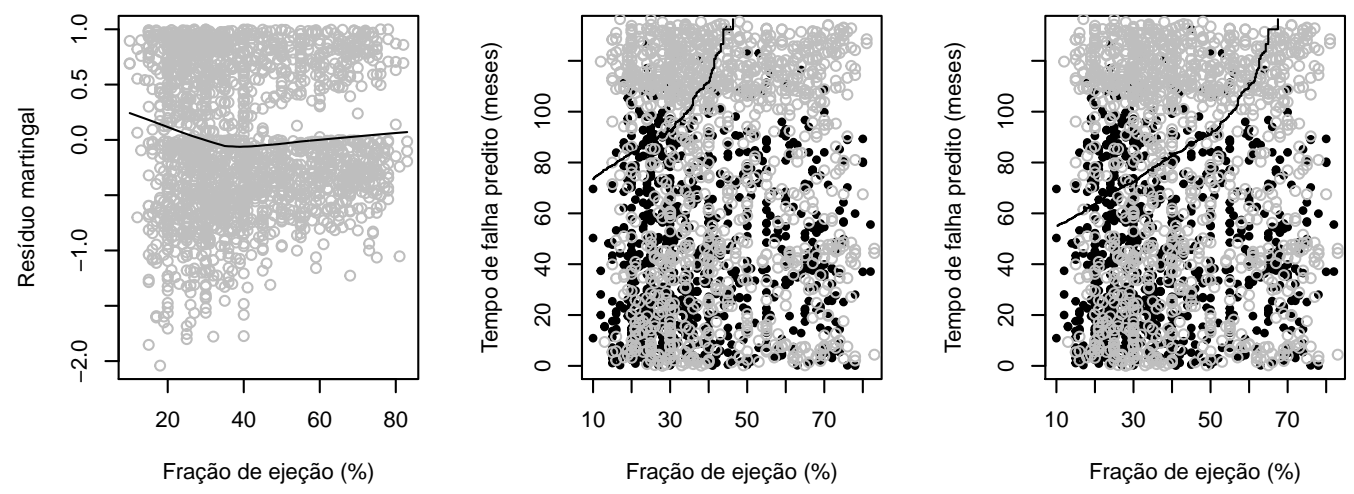

(b) Figura 2 - referente ao cenário M2

Figura 5.3: As figuras 1 e 2 apresentam os mesmos três gráficos em cada um delas. Gráfico 1 plota FE $\times$ Resíduo Martingal; Gráficos 2 e 3 apresentam os tempos de falha preditos versus FE, em que o tempo foi estimado pelo modelo de Cox de tal modo que $S(t / F E)=0.5$ e $S(t / F E)=0.6$, respectivamente. Gráficos 2 e 3 são plotados sobre o gráfico de dispersão da FE pelos tempos de falha observados, em que os pontos pretos são os óbitos e os pontos cinzas são as censuras. 

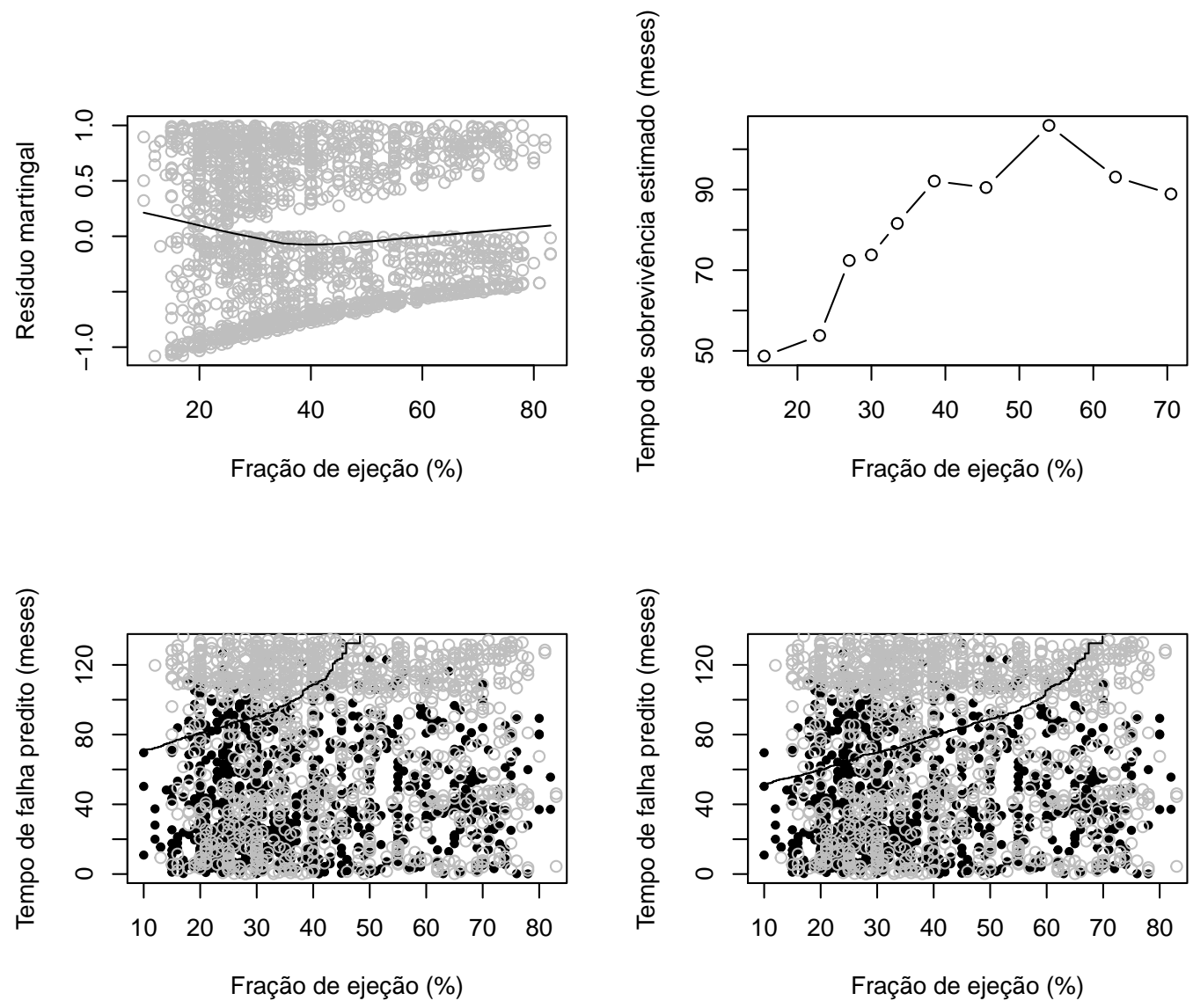

Figura 5.4: Os gráficos foram construídos com base no modelo de Cox ajustado para o cenário Uni. Gráfico 1 plota FE×Resíduo Martingal; Gráficos 2 plota o tempo de falha predito pelo método de Kaplan-Meier versus o ponto médio dos grupos definidos pelos decis da variável FE e os Gráficos 3 e 4 apresentam os tempos de falha preditos 1 e 2 versus $F E$, em que o tempo foi estimado pelo modelo de Cox de tal modo que $S(t / F E)=0.5$ e $S(t / F E)=0.6$, respectivamente. Gráficos 3 e 4 são plotados sobre o gráfico de dispersão da FE pelos tempos de falha observados, em que os pontos pretos são os óbitos e os pontos cinzas são as censuras.

A Tabela 5.10 apresenta os pontos de corte e valores- $p$ estimados a partir das estatísticas corrigidas de Contal e O'Quigley (1999) e de Jespersen. Para o cálculo do valor- $p$ no cenário multivariado pelo método de Contal O'Quigley, dez iterações foram usadas na expressão da distribuição assintótica dada por (3.8). Foram observados resíduos com valores altos, o que justifica os valores- $p$ corrigidos acima do valor desejado 0,05. Portanto, para os cenários multivariados há indícios de que a dicotomia da variável FE não é indicada, o contrário pode ser observado para o cenário Uni em ambos os métodos de correção.

\section{Correção dos resultados}

As tabelas C.12 e C.13, disponíveis no Apêndice C, apresentam os resultados de correção dos riscos relativos estimados por MDV e MPMIN, respectivamente. Os resultados foram obtidos a partir de 1000 repetições do método de validação cruzada. Para cada iteração, os dois métodos de seleção de pontos de corte eram aplicados, MDV e MPMIN, e os valores dos 
Tabela 5.8: Ponto de corte $c$ e risco relativo estimados via MDV nos 3 cenários.

\begin{tabular}{cccc} 
Cenário & Método & $\boldsymbol{c}$ & risco relativo \\
\hline \multirow{2}{*}{ M1 } & MDV1 & 33 & 0.6217 \\
& MDV2 & 26 & 0.5889 \\
M2 & MDV1 & 33 & 0.6389 \\
& MDV2 & 26 & 0.5957 \\
\multirow{2}{*}{ Uni } & MDV1 & 32 & 0.6359 \\
& MDV2 & 26 & 0.5920 \\
\hline
\end{tabular}

Tabela 5.9: Ponto de corte c, risco relativo e valor-p estimados via MPMIN nos 3 cenários.

\begin{tabular}{ccccc} 
Cenário & Método & $\boldsymbol{c}$ & risco relativo & Valor- $\boldsymbol{p}$ \\
\hline \multirow{2}{*}{ M1 } & MPMIN1 & 29 & 0.5818 & $<0.0001$ \\
& MPMIN2 & 29 & 0.5818 & $<0.0001$ \\
M2 & MPMIN1 & 29 & 0.5883 & $<0.0001$ \\
& MPMIN2 & 29 & 0.5883 & $<0.0001$ \\
& MPMIN1 & 28 & 0.5794 & $<0.0001$ \\
Uni & MPMIN2 & 28 & 0.5794 & $<0.0001$ \\
& MPMIN3 & 28 & 0.5794 & $<0.0001$ \\
\hline
\end{tabular}

Tabela 5.10: Resultados das estatísticas de teste corrigidas pelos métodos de Contal O'Quigley e Jespersen.

\begin{tabular}{cccc} 
Cenário & Método & $\boldsymbol{c}$ & valor- $\boldsymbol{p}$ \\
\hline M1 & Correção de Contal e O'Quigley Multivariado & 40 & 0.2829 \\
M2 & Correção de Contal e O'Quigley Multivariado & 40 & 0.3590 \\
& Correção de Contal e O'Quigley Univariado & 31 & 0.0003 \\
& Correção de Jespersen & 28 & $<0.0001$ \\
\hline
\end{tabular}


riscos relativos e valores- $p$ foram retidos. As tabelas apresentam as estatísticas relacionadas aos valores retidos a partir das 1000 reamostragens. As estimativas para o valor- $p$ corrigido foram inferiores a 0.01 e foram omitidas das tabelas.

MDV1 apresenta valor corrigido de $r r$ aproximadamente igual a 0.63, MDV2 aproximadamente igual a 0.57 e MPMIN aproximadamente igual a 0.60. Nota-se pequena diferença entre os riscos estimados para os métodos, considerando, então, valores corrigidos próximos a 0.6. As estimativas corrigidas dos riscos relativos para MDV1 são parecidas com as estimativas não corrigidas, enquanto que, para MDV2, as estimativas corrigidas apresentam-se menores e para MPMIN, apresentam-se maiores.

A aplicação dos métodos Lausen92 e Lausen94 para correção do valor- $p$ resultou em valores inferiores a 0.01 em todos os cenários para os três testes, MPMIN1, MPMIN2 e MPMIN3; o valor de $\epsilon$ considerado foi igual 0.2. E os riscos relativos corrigidos pelos fatores de contração heurístico, boostrap e validação cruzada, disponíveis no Apêndice C na Tabela C.18, resultaram valores próximos a 0.6 ; note que os valores de $\hat{d}$ foram próximos a 1 , o que indica que o risco relativo foi pouco superestimado pelo modelo categorizado. $\mathrm{O}$ fator de contração $v c$ foi deduzido pela média de 100 fatores. O fator de contração adhoc não foi implementado, pois os valores- $p$ estimados por MPMIN foram muito próximos a zero.

\subsubsection{Resultados para tricotomia}

A Tabela 5.11 apresenta os pontos de corte $(c 1$ e $c 2)$ e os riscos relativos $(r r 1$ e $r r 2)$ estimados por MDV (MDV1 e MDV2) e a Tabela 5.12 apresenta os pontos de corte, riscos relativos e valores- $p$ estimados por MPMIN (MPMIN1, MPMIN2 e MPMIN3). Quanto aos pontos de corte, MDV1 apresenta resultados parecidos para os três cenários, já para MDV2 não foi possível estabelecer um padrão. MPMIN apresenta os mesmos valores de $c 1$ e $c 2$ nos cenários M1 e Uni, já o cenário M2 apresenta um valor mais alto para $c 2$. Como na dicotomia, MPMIN apresenta menor variabilidade nos resultados entre os cenários.

Em relação aos riscos relativos, MDV1 apresenta estimativas levemente mais altas para $r r 1$ do que MDV2 e, consequentemente, maior diferença entre os riscos $(r r 1-r r 2)$. As estimativas de $r r 1$ feitas por MPMIN são próximas às estimativas de MDV1, mas os valores de $\operatorname{rr} 2$ são mais baixos e, portanto, MPMIN apresenta maior diferença entre os riscos. Do ponto de vista dos riscos, o melhor modelo foi ajustado no cenário Uni para os pontos de corte estimados pelo MPMIN $c 1=25$ e $c 2=30$.

\section{Correção dos resultados}

As tabelas C.16 e C.17, disponíveis no Apêndice C, apresentam os resultados para correção dos riscos relativos estimados por MDV e riscos relativos e valores- $p$ estimados por MPMIN, respectivamente. Os resultados foram obtidos por meio de 1000 repetições do método de validação cruzada. Para cada iteração, os dois métodos de seleção de pontos de corte foram aplicados, MDV e MPMIN, e os valores dos riscos relativos, $r r 1$ e $r r 2$, e valores- $p$ fo- 
Tabela 5.11: Vetor de pontos de corte $c^{\prime}=(c 1, c 2)$, riscos relativos 1 e 2 estimados via $M D V$ nos 3 cenários.

\begin{tabular}{cccccc} 
Cenário & Método & $\boldsymbol{c 1}$ & $\boldsymbol{c 2}$ & risco relativo 1 & risco relativo 2 \\
\hline \multirow{2}{*}{ M1 } & MDV1 & 32 & 37 & 0.7372 & 0.6258 \\
& MDV2 & 26 & 56 & 0.6279 & 0.5471 \\
M2 & MDV1 & 32 & 37 & 0.7438 & 0.6159 \\
& MDV2 & 31 & 45 & 0.6382 & 0.6051 \\
\multirow{2}{*}{ Uni } & MDV1 & 33 & 39 & 0.7062 & 0.6220 \\
& MDV2 & 32 & 57 & 0.6531 & 0.6044 \\
\hline
\end{tabular}

Tabela 5.12: Vetor de pontos de corte $c^{\prime}=(c 1, c 2)$, riscos relativos 1 e 2 e valor- $p$ estimados via MPMIN nos 3 cenários.

\begin{tabular}{ccccccc} 
Cenário & Método & $\boldsymbol{c 1}$ & $\boldsymbol{c 2}$ & risco relativo 1 & risco relativo 2 & Valor- $\boldsymbol{p}$ \\
\hline \multirow{2}{*}{ M1 } & MPMIN1 & 25 & 30 & 0.7468 & 0.5491 & $<0.0001$ \\
& MPMIN2 & 25 & 30 & 0.7468 & 0.5491 & $<0.0001$ \\
\multirow{2}{*}{ M2 } & MPMIN1 & 25 & 35 & 0.6818 & 0.5199 & $<0.0001$ \\
& MPMIN2 & 25 & 35 & 0.6818 & 0.5199 & $<0.0001$ \\
& MPMIN1 & 25 & 30 & 0.7585 & 0.5361 & $<0.0001$ \\
\multirow{2}{*}{ Uni } & MPMIN2 & 25 & 30 & 0.7585 & 0.5361 & $<0.0001$ \\
& MPMIN3 & 25 & 30 & 0.7585 & 0.5361 & $<0.0001$ \\
\hline
\end{tabular}

ram retidos. As tabelas apresentam as estatísticas relacionadas aos valores retidos a partir das 1000 reamostragens. As estimativas para o valor- $p$ corrigido foram inferiores a $0.01 \mathrm{e}$ foram omitidas das tabelas

Para MDV1 e MDV2, as estimativas para $r r 1$ são próximas a 0.64 , com desvio padrão igual a 0.04 , para os três cenários; para $r r 2$ as estatísticas são próximas a 0.60 , com desvio de 0.03, aproximadamente. Para MPMIN1, as estimativas de $r r 1$ são de aproximadamente 0.66 (desvio igual a 0.03) e para $r r 2$ são de aproximadamente 0.56 (desvio igual a 0.02) para todos os cenários; os métodos MPMIN2 e MPMIN3 apresentam estatísticas de $r r 1$ próximas a 0.62 (desvio igual a 0.03) e as estimativas para $r r 2$ são similares entre os cenários, com valores próximos a 0.57 com erro de, aproximadamente, 0.03 .

Observe que as correções indicam pouca diferença entre os grupos com FE limítrofe e preservada, exceto para MPMIN1. As correções tornaram as estimativas de MDV1 e MDV2 próximas, além disso, essas estimativas foram próximas às correções feitas no caso em que as amostras ainda não haviam sido ajustadas, nos cenários M2, M3 e Uni.

\subsubsection{Validação dos pontos de corte, valores- $p$ e riscos relativos}

Assim como na Seção 5.4, a fim de identificar estimativas de pontos de corte e de riscos relativos robustas à variabilidade dos dados, o método de reamostragem bootstrap foi aplicado aos dados. Foram feitas 2000 reamostragens para cada combinação de método de seleção de ponto de corte (MDV e MPMIN), cenário (M1, M2 e Uni) e quantidade de grupos (dicotomia e tricotomia), e foram extraídos os respectivos valores dos pontos de corte, 
valores- $p$ e riscos relativos estimados a partir do modelo categorizado. As modas de cada valor estimado foram escolhidas como valor validado.

Para dicotomia, as tabelas C.10 e C.11 e as figuras D.8 e D.9, disponíveis nos apêndices C e D, estão relacionadas às amostras bootstrap calculadas sobre os métodos MDV e MPMIN, respectivamente. Para o caso de tricotomia, a Tabela C.14 e as figuras D.10 e D.11, também disponíveis em (C) e (D), estão relacionadas às amostras bootstrap calculadas sobre os métodos MDV1 e MDV2, e a Tabela C.15 e as figuras D.12, D.13 e D.14 estão relacionadas às amostras bootstrap calculadas sobre os métodos MPMIN1, MPMIN2 e MPMIN3. Todos os valores- $p$ estimados nas amostras bootstrap em todas as combinações foram inferiores a 0.01 e, portanto, os resultados foram omitidos das tabelas a seguir.

Tabela 5.13: Moda dos 2000 pontos de corte e moda dos 2000 riscos relativos estimados pelos métodos MDV e MPMIN nas amostras bootstrap, para os cenários M1, M2 e Uni, no caso de dicotomia.

\begin{tabular}{ccccccc} 
& & \multicolumn{5}{c}{ Métodos } \\
\cline { 3 - 7 } Medida & Cenário & MDV1 & MDV2 & MPMIN1 & MPMIN2 & MPMIN3 \\
\hline \multirow{2}{*}{ c } & M1 & 35 & 31 & 29 & 29 & - \\
& M2 & 35 & 31 & 29 & 29 & - \\
& Uni & 36 & 31 & 30 & 28 & 28 \\
\multirow{3}{*}{ rr } & M1 & 0.63 & 0.61 & 0.55 & 0.56 & - \\
& M2 & 0.62 & 0.61 & 0.58 & 0.58 & - \\
& Uni & 0.62 & 0.62 & 0.57 & 0.57 ou 0.58 & 0.57 \\
\hline
\end{tabular}

Ao avaliar a Tabela 5.13 e as outras tabelas e figuras disponíveis nos apêndices sobre os valores obtidos nas amostras bootstrap para dicotomia, observa-se que os valores de ponto de corte $(c)$ estimados por MDV se concentram entre os valores de 30 a 35 e os valores estimados por MPMIN se concentram no valor 29, aproximadamente. Os histogramas dos riscos relativos $(r r)$ se assemelham à curva da distribuição Normal; para MDV as curvas são aproximadamente centradas em 0.62 e para MPMIN, são aproximadamente centradas em 0.57. Os desvios padrão dos valores obtidos para $c$ são aproximadamente iguais a 4 para MDV e iguais a 3 para MPMIN entre os cenários; para os riscos relativos, os desvios são aproximadamente iguais a 0.05 para MDV e 0.04 para MPMIN.

Os métodos MDV1 e MPMIN retornam valores validados de $c$ e $r r$ próximos aos valores anteriormente estimados, apresentados nas tabelas 5.8 e 5.9. O método MDV2 estimou anteriormente $c=26$ e os valores validados foram iguais a 31 em todos cenários; ainda assim, MDV2 permanece tendo estimativas de $c$ mais próximas de MPMIN do que de MDV1. Ao avaliar o qualidade dos pontos de corte estimados segundo o critério do risco relativo, o ponto de corte selecionado é $c=29$ estimado por MPMIN1 no cenário M1 e o risco relativo estimado foi de 0.55 .

Ao avaliar a Tabela 5.14 e as outras tabelas e figuras disponíveis nos apêndices sobre os valores obtidos nas amostras bootstrap para tricotomia, observa-se que os valores estimados do ponto de corte $c 1$ se concentram próximos a 32 para MDV e próximos a 25 para MPMIN 
Tabela 5.14: Moda dos 2000 pontos de corte e 2000 riscos relativos estimados pelos métodos MDV e MPMIN nas amostras bootstrap, para os cenários M1, M2 e Uni, no caso de tricotomia.

\begin{tabular}{ccccccc} 
& & \multicolumn{5}{c}{ Métodos } \\
\cline { 3 - 7 } Medida & Cenário & MDV1 & MDV2 & MPMIN1 & MPMIN2 & MPMIN3 \\
\hline \multirow{2}{*}{ c1 } & M1 & 32 & 31 & 25 & 25 & - \\
& M2 & 32 & 31 & 25 & 25 & - \\
& Uni & 32 & 32 & 25 & 25 & 25 \\
rr1 & M1 & 0.69 & 0.64 & 0.69 & 0.69 & - \\
& M2 & 0.66 & 0.64 & 0.66 & 0.65 ou 0.68 & - \\
& Uni & 0.66 & 0.64 & 0.67 & 0.67 & 0.66 \\
\multirow{2}{*}{ c2 } & M1 & 45 & 45 & 30 & 35 & - \\
& M2 & 45 & 45 & 30 & 35 & - \\
& Uni & 45 & 45 ou 56 & 30 & 30 & 30 \\
\cline { 3 - 7 } rr2 & M1 & 0.59 & 0.60 & 0.49 & 0.51 & - \\
& M2 & 0.58 & 0.60 & 0.49 & 0.51 & - \\
& Uni & 0.57 ou 0.61 & 0.61 & 0.52 & 0.52 & 0.52 \\
\hline
\end{tabular}

e, para $c 2$, os histogramas se concentram nos pontos 45 e 54 para MDV e entre os pontos 30 e 35 para MPMIN. Apesar de $c 1$ ser próximo a 32 para MDV, os histogramas para $c 1$ também apresentam um pico no valor 25, que é o valor corrigido para MPMIN.

Quanto aos riscos relativos, ao avaliar os histogramas que apresentam uma silhueta similar à curva da distribuição Normal, as estimativas de $r r 1$ para ambos os métodos, independente do cenário, são aproximadamente iguais a 0.66 , já os valores de $r r 2$ se concentram em 0.6 para MDV e em 0.52 para MPMIN. O método MPMIN sugere maior diferença entre os riscos dos grupos gerados pelos pontos de corte $c 1$ e $c 2$.

Os valores de $c 1$ e $c 2$ validados tem menor variabilidade que seus respectivos valores não validados disponíveis nas tabelas 5.11 e 5.12 entre os cenários e para ambos os métodos. Os valores de $r r 1$ e $r r 2$ validados para MDV continuam muito parecidos com os valores não validados, enquanto que, para MPMIN, os valores validados para $r r 1$ indicam uma redução de aproximadamente 0.1 em relação aos não validados e os valores validados de $r r 2$ são similares aos não validados. Note que após a validação dos dados, a diferença de riscos entre os grupos gerados pelos pontos de corte estimados via MPMIN foi reduzida.

Ao avaliar o qualidade dos pontos de corte estimados segundo o critério do risco relativo, os pontos de corte selecionados são: $c 1=25$ e $c 2=30$ estimados por MPMIN1 no cenário $\mathrm{M} 1$, os riscos relativos estimados foram de $r r 1=0.69$ e $r r 2=0.49$.

\subsection{Modelos finais}

As tabelas 5.16 e 5.17 apresentam os pontos de corte estimados pelos métodos MDV, MPMIN, estatísticas corrigidas de Contal O'Quigley (dicotomia) e Jespersen (dicotomia), média (dicotomia), mediana (dicotomia) e tercis (tricotomia) da fração de ejeção e, também, consideram os pontos de corte validados pelo método boot a partir de MDV e MPMIN. 
A fim de comparar os modelos ajustados pela fração de ejeção categorizada pelos pontos de corte estimados (ou validados) foram usadas as estatísticas: variação explicada $R^{2}$ e o $A I C$ (Akaike Information Criterion) definidos no Apêndice B, a estatística qui-quadrado Score e seu respectivo valor- $p$, e os riscos relativos $(r r)$ estimados a partir do modelo categorizado. Valores altos para $R^{2}$ e $\chi^{2}$, e valores baixos para AIC e valor- $p$ indicam melhor ajuste dos dados pelo modelo.

Cada cenário apresenta um valor diferente para o número de parâmetros estimados no modelo (graus de liberdade da estatística Score). Um modelo com mais variáveis explicativas pode parecer ter um ajuste melhor por possuir mais termos, influenciando os valores de $R^{2}$ e $\chi^{2}$. A fim de comparar os modelos entre os cenários, a única estatística levada em conta é a AIC, que é ajustada pelos graus de liberdade do modelo. E, então, as outras estatísticas de comparação podem ser usadas dentro de cada cenário para avaliar o melhor modelo ajustado. Todos os valores- $p$ são inferiores a 0.01 e não são úteis na classificação dos modelos e, também, as estimativas de $R^{2}$ foram próximas dentro de cada cenário e, portanto, são pouco informativas.

Olhando para o cenário M1, o ponto de corte que resultou no melhor ajuste para dicotomia, segundo AIC, $\chi^{2}$ e $r r$, foi $c=29$ estimado por MPMIN e MPMIN*; para tricotomia, $c 1=25$ e $c 2=35$ estimados por MPMIN2* resultou no melhor ajuste segundo AIC, e os pontos $c 1=25$ e $c 2=30$ estimados por MPMIN e MPMIN1*, segundo $\chi^{2}$ e $r r$.

Para o cenário M2, o ponto de corte que resultou no melhor ajuste para dicotomia, segundo AIC, $\chi^{2}$ e $r r$, foi $c=29$ estimado por MPMIN e MPMIN*; para tricotomia, $c=$ $(25,35)$ estimado por MPMIN e MPMIN2* resultou no melhor ajuste segundo AIC e $\chi^{2}$, e os pontos $c 1=25$ e $c 2=30$ estimados por MPMIN1*, segundo $r r$.

No cenário Uni, os pontos de corte que resultaram no melhor ajuste para dicotomia, segundo AIC, $\chi^{2}$ e $r r$, foi $c=28$ estimado por MPMIN, MPMIN2*, MPMIN3* e Jespersen e, para tricotomia, $c=(25,30)$ estimados por MPMIN e MPMIN*.

De modo geral, para os três cenários, o melhor ajuste para o caso de dicotomia foi feito na presença da variável dicotomizada por $c$ aproximadamente igual a 29, estimado por MPMIN, com risco relativo próximo a 0.58 ; e para o caso de tricotomia, foi feito para $c=(25,30)$, estimados por MPMIN, com rr1 próximo a 0.75 (exceto para M2, próximo a 0.68 ) e $r r 2$ próximo a 0.53 .

Segundo o critério AIC, ao avaliar a Tabela 5.16, o modelo ajustado na presença da variável dicotomizada por $c=29$ no cenário M1 apresenta o melhor desempenho preditivo, em que $c$ foi estimado pelos métodos MPMIN e MPMIN*. Ao avaliar a Tabela 5.17, o modelo na presença da variável tricotomizada por $c 1=25$ e $c 2=35$ estimados por MPMIN2* no cenário M1 apresenta o melhor desempenho preditivo. Ao avaliar as duas tabelas, com os resultados de dicotomia e tricotomia, o melhor ajuste é observado para o modelo na presença da variável FE tricotomizada pelo par de pontos de corte $c=(25,35) \quad(\mathrm{AIC}=12326.59)$ no cenário M1.

Ao avaliar as curvas de Kaplan-Meier apresentadas na Figura 5.5 e os teste logrank e 
Tabela 5.15: Resultados dos testes logrank e Wilcoxon utilizados para as comparações dos grupos de pacientes com ICFER, ICFEL e ICFEP, dois a dois.

\begin{tabular}{ccccc} 
Grupos comparados & Teste logrank & Valor p & Teste Wilcoxon & Valor p \\
\hline Todos & 66.4 & $<0.01$ & 67.0 & $<0.01$ \\
ICFER e ICFEL & 18.3 & $<0.01$ & 17.1 & $<0.01$ \\
ICFEL e ICFEP & 10.4 & $<0.01$ & 11.5 & $<0.01$ \\
ICFER e ICFEP & 67.1 & $<0.01$ & 67.8 & $<0.01$ \\
\hline
\end{tabular}

Wilcoxon apresentados na Tabela 5.15 verifica-se que os pontos de corte selecionados pelo método MPMIN2* no cenário M1, $c=(25,35)$, produziram categorias com riscos estatisticamente distintos. Note que tais pontos apresentaram ser mais eficientes para separação dos indivíduos segundo a FE do que os pontos inicialmente sugeridos $c=(45,55)$, avaliados no Capítulo 2.

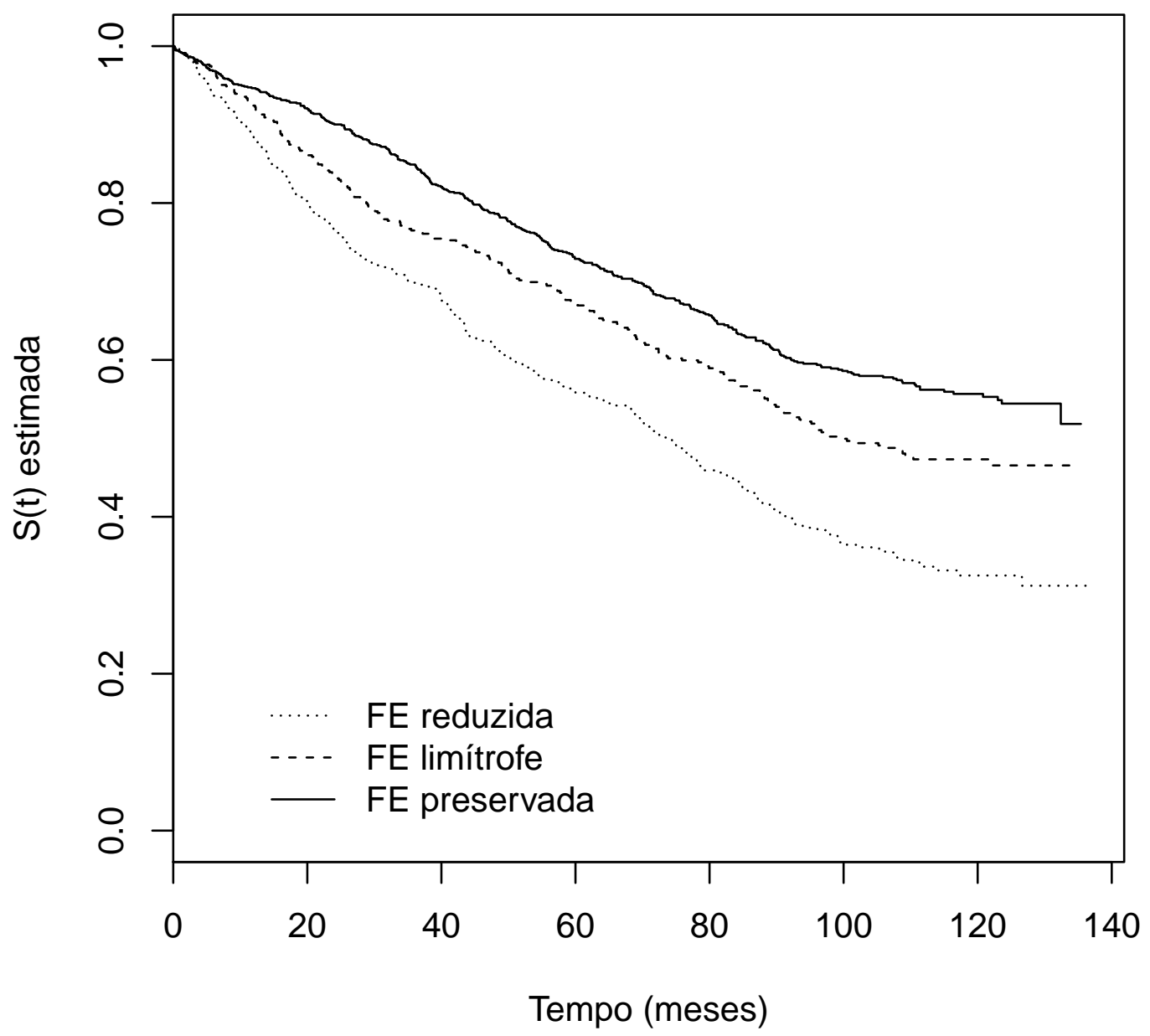

Figura 5.5: Curvas de Kaplan-Meier para as três categorias de FE definidas pelos pontos de corte $25 \%$ e $35 \%$ selecionados por AIC. 
Tabela 5.16: Para cada ponto de corte estimado c, os valores da estatística Score $\chi^{2}$ e seus respectivos graus de liberdade (g.l.) e valor-p, variação explicada $R^{2}$, AIC e risco relativo (rr) foram calculados no modelo categorizado. Os métodos MDV, MPMIN, MDV ${ }^{*}, M P M I N^{*}$, estatísticas corrigidas de Contal O'Quigley e Jespersen, além da média e mediana da FE foram avaliados nos 3 cenários propostos para o caso em que as amostras foram homogeneizadas entre os cenários.

\begin{tabular}{|c|c|c|c|c|c|c|c|c|}
\hline Cenário & Método & $c$ & $R^{2}$ & AIC & $\chi^{2}$ & g.l. & valor- $p$ & $\mathbf{r r}$ \\
\hline \multirow{9}{*}{ M1 } & MDV1 & 33 & 0.10 & 12341.91 & 216.63 & \multirow{9}{*}{12} & $<0.01$ & 0.62 \\
\hline & MDV2 & 26 & 0.10 & 12339.95 & 222.76 & & $<0.01$ & 0.59 \\
\hline & MPMIN & 29 & 0.10 & 12331.30 & 230.47 & & $<0.01$ & 0.58 \\
\hline & Mediana & 36 & 0.09 & 12345.03 & 212.81 & & $<0.01$ & 0.63 \\
\hline & Média & 40.5 & 0.09 & 12354.31 & 202.16 & & $<0.01$ & 0.66 \\
\hline & Contal & 40 & 0.09 & 12354.31 & 202.16 & & $<0.01$ & 0.66 \\
\hline & $\mathrm{MDV}^{*}$ & 35 & 0.10 & 12342.23 & 215.62 & & $<0.01$ & 0.62 \\
\hline & MDV2* & 31 & 0.10 & 12334.90 & 224.39 & & $<0.01$ & 0.60 \\
\hline & MPMIN* & 29 & 0.10 & 12331.30 & 230.47 & & $<0.01$ & 0.58 \\
\hline \multirow{9}{*}{ M2 } & MDV1 & 33 & 0.09 & 12351.28 & 199.48 & \multirow{9}{*}{11} & $<0.01$ & 0.64 \\
\hline & MDV2 & 26 & 0.09 & 12346.51 & 207.70 & & $<0.01$ & 0.60 \\
\hline & MPMIN & 29 & 0.10 & 12338.18 & 214.95 & & $<0.01$ & 0.59 \\
\hline & Mediana & 40 & 0.09 & 12353.21 & 196.60 & & $<0.01$ & 0.64 \\
\hline & Média & 36 & 0.09 & 12364.48 & 185.11 & & $<0.01$ & 0.68 \\
\hline & Contal & 40.5 & 0.09 & 12364.48 & 185.11 & & $<0.01$ & 0.68 \\
\hline & MDV1* & 35 & 0.09 & 12351.32 & 198.65 & & $<0.01$ & 0.64 \\
\hline & MDV2* & 31 & 0.09 & 12345.61 & 206.01 & & $<0.01$ & 0.62 \\
\hline & MPMIN* & 29 & 0.10 & 12338.18 & 214.95 & & $<0.01$ & 0.59 \\
\hline \multirow{11}{*}{ Uni } & MDV1 & 32 & 0.02 & 12485.71 & 45.73 & \multirow{11}{*}{1} & $<0.01$ & 0.64 \\
\hline & MDV2 & 26 & 0.02 & 12481.71 & 53.45 & & $<0.01$ & 0.59 \\
\hline & MPMIN & 28 & 0.03 & 12473.05 & 62.24 & & $<0.01$ & 0.58 \\
\hline & Mediana & 36 & 0.02 & 12488.04 & 42.19 & & $<0.01$ & 0.64 \\
\hline & Média & 40.5 & 0.02 & 12496.27 & 33.17 & & $<0.01$ & 0.66 \\
\hline & Contal & 31 & 0.02 & 12479.63 & 52.38 & & $<0.01$ & 0.62 \\
\hline & Jespersen & 28 & 0.03 & 12473.05 & 62.24 & & $<0.01$ & 0.58 \\
\hline & $\mathrm{MDV}^{*}$ & 36 & 0.02 & 12488.04 & 42.19 & & $<0.01$ & 0.64 \\
\hline & $\operatorname{MDV} 2 *$ & 31 & 0.02 & 12479.63 & 52.38 & & $<0.01$ & 0.62 \\
\hline & MPMIN1* & 30 & 0.03 & 12473.63 & 59.30 & & $<0.01$ & 0.60 \\
\hline & MPMIN2*MPMIN3* & 28 & 0.03 & 12473.05 & 62.24 & & $<0.01$ & 0.58 \\
\hline
\end{tabular}

i MDV*, MPMIN1*, MPMIN2* e MPMIN3* referenciam o método de validação bootstrap usando os métodos MDV, MPMIN1, MPMIN2 e MPMIN3, respectivamente.

${ }^{\text {ii }}$ A estatística qui-quadrado Score e o valor-p referem-se ao teste global de ajuste do modelo. 
Tabela 5.17: Para cada par de pontos de corte estimado c1 e c2, os valores da estatística Score $\chi^{2}$ e seus respectivos graus de liberdade (g.l.) e valor-p, variação explicada $R^{2}$, AIC e riscos relativos (rr1 e rr2) foram calculados no modelo categorizado. Os métodos MDV, MPMIN, $M D V^{*}, M P M I N^{*}$ e os tercis da FE foram avaliados nos 3 cenários propostos para o caso em que as amostras foram homogeneizadas entre os cenários.

\begin{tabular}{|c|c|c|c|c|c|c|c|c|c|c|}
\hline Cenário & Método & $c 1$ & $c 2$ & $R^{2}$ & AIC & $\chi^{2}$ & g.l. & valor- $p$ & rr1 & rr2 \\
\hline \multirow{8}{*}{ M1 } & MDV1 & 40 & 45 & 0.09 & 12355.55 & 202.50 & \multirow{8}{*}{13} & $<0.01$ & 0.58 & 0.67 \\
\hline & MDV2 & 35 & 54 & 0.10 & 12344.23 & 215.63 & & $<0.01$ & 0.62 & 0.62 \\
\hline & MPMIN & 25 & 30 & 0.10 & 12326.74 & 237.86 & & $<0.01$ & 0.75 & 0.53 \\
\hline & Tercil & 30 & 46 & 0.10 & 12334.01 & 228.19 & & $<0.01$ & 0.62 & 0.57 \\
\hline & MDV1* & 32 & 45 & 0.10 & 12343.72 & 216.96 & & $<0.01$ & 0.65 & 0.60 \\
\hline & MDV2* & 31 & 45 & 0.10 & 12336.55 & 224.72 & & $<0.01$ & 0.62 & 0.59 \\
\hline & MPMIN1* & 25 & 30 & 0.10 & 12326.74 & 237.86 & & $<0.01$ & 0.75 & 0.53 \\
\hline & MPMIN2* & 25 & 35 & 0.10 & 12326.59 & 237.69 & & $<0.01$ & 0.69 & 0.51 \\
\hline \multirow{8}{*}{ M2 } & MDV1 & 34 & 56 & 0.09 & 12354.18 & 198.35 & \multirow{8}{*}{12} & $<0.01$ & 0.66 & 0.61 \\
\hline & MDV2 & 34 & 51 & 0.09 & 12353.44 & 198.99 & & $<0.01$ & 0.68 & 0.61 \\
\hline & MPMIN & 25 & 35 & 0.10 & 12335.02 & 220.62 & & $<0.01$ & 0.68 & 0.52 \\
\hline & Tercil & 30 & 46 & 0.10 & 12344.25 & 210.04 & & $<0.01$ & 0.63 & 0.59 \\
\hline & MDV1* & 32 & 45 & 0.09 & 12353.09 & 199.95 & & $<0.01$ & 0.66 & 0.62 \\
\hline & MDV2* & 31 & 45 & 0.09 & 12347.30 & 206.24 & & $<0.01$ & 0.64 & 0.61 \\
\hline & MPMIN1* & 25 & 30 & 0.10 & 12335.61 & 220.58 & & $<0.01$ & 0.73 & 0.54 \\
\hline & MPMIN2* & 25 & 35 & 0.10 & 12335.02 & 220.62 & & $<0.01$ & 0.68 & 0.52 \\
\hline \multirow{8}{*}{ Uni } & MDV1 & 34 & 52 & 0.02 & 12490.66 & 42.16 & \multirow{8}{*}{2} & $<0.01$ & 0.66 & 0.63 \\
\hline & MDV2 & 33 & 53 & 0.02 & 12487.97 & 45.18 & & $<0.01$ & 0.65 & 0.62 \\
\hline & MPMIN & 25 & 30 & 0.03 & 12468.28 & 69.11 & & $<0.01$ & 0.76 & 0.54 \\
\hline & Tercil & 30 & 46 & 0.03 & 12475.04 & 59.77 & & $<0.01$ & 0.62 & 0.58 \\
\hline & MDV1* & 32 & 55 & 0.02 & 12486.67 & 46.57 & & $<0.01$ & 0.66 & 0.60 \\
\hline & MDV2* & 32 & 45 & 0.02 & 12487.23 & 46.13 & & $<0.01$ & 0.66 & 0.62 \\
\hline & $\mathrm{MDV} 2 * *$ & 32 & 56 & 0.02 & 12487.48 & 45.92 & & $<0.01$ & 0.65 & 0.62 \\
\hline & MPMIN* & 25 & 30 & 0.03 & 12468.28 & 69.11 & & $<0.01$ & 0.76 & 0.54 \\
\hline
\end{tabular}

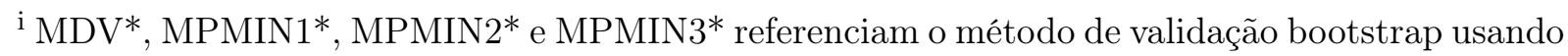
os métodos MDV, MPMIN1, MPMIN2 e MPMIN3, respectivamente.

ii A estatística qui-quadrado Score e o valor-p referem-se ao teste global de ajuste do modelo.

iii MDV2** foi usado pois a amostra bootstrap gerada pelo método MDV2 no cenário Uni apontou duas modas para o ponto $\mathrm{c} 2$. 


\subsection{Discussão}

Os diferentes cenários e métodos de seleção de pontos de corte e de correção e validação das estimativas foram avaliados em dois casos:

Caso 1: Os modelos nos cenários M1, M2, M3 foram ajustados com diferentes variáveis significativas e amostras de tamanhos diferentes, devido ao problema de dados faltantes. A mesma amostra foi utilizada para os cenários M1 e Uni.

Caso 2: Os modelos nos cenários M1 e M2 foram ajustados com as mesmas variáveis significativas e mesma amostra, para facilitar a comparação entre os métodos. O cenário Uni também foi avaliado nessa mesma amostra.

A análise gráfica foi importante para restringir a busca pelos pontos de corte para a variável fração de ejeção, além de indicar as regiões mais prováveis para encontrá-los. Os gráficos reduziram as buscas para os $60 \%$ centrais dos valores da variável, agilizando o processo de busca para os métodos que exigem comparações múltiplas e contribuindo para que a taxa do erro tipo I não fique tão acima da esperada 0.05.

As estimativas para os pontos de corte dadas por MDV1, MDV2 e MPMIN diferem entre si (para dicotomia e tricotomia). Os resultados para MDV1 e MDV2 diferem como esperado, afinal, consideram pontos de corte associados às verossimilhanças maior e menor que a verossimilhança do modelo contínuo. Os resultados para MPMIN1, MPMIN2 e MPMIN3 são muito próximos e, em muitos casos, são iguais quando avaliados dentro de um mesmo cenário, o que também é esperado, dada a equivalência assintótica dos três respectivos testes: TRV, Wald e Score e, por isso, frequentemente será referenciado o resultado obtido por MPMIN apenas.

\section{Dicotomia:}

$M D V$ : Caso 1: As estimativas para $c$ e $r r$ são mais altas para o cenário M1 do que para os demais cenários. MDV2 apresenta estimativas (e validações) para $c$ mais baixas do que MDV1, dentro de cada cenário. Os valores validados são bem próximos aos valores estimados no cenário M2, para o restante dos cenários observa-se grandes diferenças como, por exemplo, no cenário M1, em que $c$ passou de 26 (estimado) para 35 (validado). Os riscos relativos validados, ao contrário dos estimados, ficam mais parecidos entre os cenários, exceto para M1. Caso 2: As estimativas e (validações) para $c$ e $r r$ parecem ser menos sensíveis à troca de cenário, para MDV1 e MDV2. Os valores de $r r$ são próximos a 0.61 . Nos casos 1 e 2, os riscos relativos e valores- $p$ corrigidos pelos métodos $v c$ ou $f c$ são muito próximos aos riscos e valores- $p$ validados.

MPMIN: Os casos 1 e 2 apresentam estimativas e validações de $c$ e $r r$ muito próximas, 
exceto por M1 que apresenta estimativas e validações para $r r$ mais altas no caso 1 . Nos casos 1 e 2, além dos riscos relativos e valores- $p$ validados e corrigidos pelos métodos $v c$ ou $f c$ serem muito próximos entre si, eles também são parecidos com os riscos e valores- $p$ validados e corrigidos por MDV.

\section{Tricotomia:}

MDV: Caso 1: MDV1 e MDV2 apresentam maior quantidade de possíveis vetores de pontos de corte, $\boldsymbol{c}$, do que MPMIN. Os valores de $\boldsymbol{c}$ validados foram distantes dos pontos estimados. O cenário M1 apresenta riscos relativos estimados (e validados) mais altos em comparação aos demais cenários. Os riscos relativos validados são parecidos entre os cenários M2, M3 e Uni, ao contrário dos riscos estimados. Os valores de $r r 2$ corrigidos são um pouco mais altos do que os validados. As validações e correções apresentam valores- $p$ menores ou iguais a 0.01. Caso 2: As estimativas de $\boldsymbol{c}$ e dos riscos para MDV1 são pouco sensíveis à troca de cenário, o mesmo pode ser observado para MDV2. Os valores validados acentuam a semelhança das estimativas entre os cenários. As correções feitas pelo método vc para os riscos relativos e valores- $p$ foram próximas aos valores validados.

MPMIN: Caso 1: Os métodos MPMIN1, MPMIN2 e MPMIN3 apresentam resultados parecidos para $\boldsymbol{c}$ dentro cada cenário, inclusive para os resultados validados. As estimativas e validações para $r r 1$ foram mais altas para os cenários M1 e Uni, e para $r r 2$, mais altas para o cenário M1. As diferenças entre os riscos estimados ou validados $r r 1$ e $r r 2$ são menores em M1 e maiores em Uni. Os valores de $r r 2$ corrigidos pelo método $v c$ são mais altos que os validados. Os valores- $p$ corrigidos por $v c$ são menores ou iguais a 0.01. Caso 2: As estimativas para $\boldsymbol{c}$ são semelhantes entre os métodos e os cenários. Em relação aos valores corrigidos por $v c$, os resultados se comportam da mesma forma que no caso 1.

De modo geral, MPMIN foi menos sensível às trocas de cenários e à validação nas estimativas de pontos de corte do que MDV, tanto para dicotomia quanto para tricotomia, nos casos 1 e 2 . Os riscos relativos corrigidos e validados apresentam valores muito próximos a 0.60 no caso de dicotomia, para tricotomia há maior variabilidade. O caso 2 apresenta estimativas mais robustas à troca de cenários dentro de cada método de categorização.

No caso 1, o cenário M1 apresenta estimativas de pontos de corte ou riscos relativos mais altos que nos demais cenários, este fato pode ter sido conduzido pelo maior número de covariáveis ajustadas ao modelo, aumentando a quantidade de parâmetros estimados e introduzindo maior erro às estimativas. No caso 2 , foi usada a mesma amostra em todas as situações, e os cenários M1 e M2 tiveram as mesmas variáveis ajustadas ao modelo, com uma única variável originalmente contínua e, portanto, houve pouca diferença entre eles, o que pode justificar os resultados parecidos entre os cenários dentro de cada método. Nos casos 1 e 2, nos métodos que envolviam reamostragens, $c v$ e boot, as estimativas de $c$ e $r r$ 
no cenário M1 apresentam maiores desvios que nos demais cenários.

Os valores validados apresentam uma certa convergência dos resultados entre os cenários para $c$ e $r r$, para os métodos MDV1, MDV2 e MPMIN. Os valores estimados, validados e corrigidos para $c 1$ apresentam valores próximos dentro de cada método e os valores de $c 2$ variam entre 30 e 57, que foram os valores de mudança de inclinação nas curvas dos gráficos apresentados.

As estimações, validações e correções indicam valores- $p \leq 0.01$ em todos os cenários, para todos os métodos, nos casos 1 e 2 . Apenas as estatísticas corrigidas baseadas no teste de Contal O'Quigley nos cenários multivariados indicam que a dicotomia da FE não é indicada ao apresentar valores- $p \geq 0.28$, indicando que a variável FE binária não tem efeito sobre o tempo de óbito dos pacientes com IC.

Observe que os melhores modelos selecionados pelos critérios AIC, $\chi^{2}$ ou riscos relativos foram ajustados na presença da FE categorizada a partir de pontos de corte estimados ou validados por MPMIN (ver Seção 5.6). Os pontos de corte estimados por MPMIN conduzem a riscos relativos mais distantes do que os pontos estimados por MDV, o que pode ser indício de que MPMIN pode ser mais eficiente para selecionar pontos de corte para categorização de variáveis de modo que os grupos gerados tenham riscos estatisticamente distintos do que MDV.

\subsubsection{Pesquisas futuras}

Baseando-se nos resultados obtidos durante o trabalho, seguem algumas pretensões de pesquisas futuras:

- Comparar os métodos MDV e MPMIN via estudo de simulação.

- Estudar sobre possíveis ajustes nos métodos de seleção de pontos de corte a fim de enfatizar a diferença entre os grupos de principal interesse.

- Se aprofundar em métodos de correção das estimativas feitas a partir de modelos categorizados para o caso de politomia.

- Aplicar os métodos citados na Seção 3.3.4 e verificar possíveis extensões para o caso de tricotomia na presença de censura.

- Verificar métodos para resolução do problema de dados faltantes. 


\section{Apêndice A}

\section{Testes da razão de verossimilhanças, Wald e Score}

Outros possíveis testes indicados para estimação dos pontos de corte que permitem a inserção de outras covariáveis significativas na análise, são os testes de razão de verossimilhança, Wald e score (Klein e Wu (2004) e Holländer et al. (2004)). Para dedução dos três testes citados é necessário utilizar-se de resultados assintóticos e métodos para amostras grandes.

Então, suponha uma amostra aleatória obtida de um modelo semi-paramétrico de Cox dada por $\left(t_{i}, \delta_{i}, x_{i}\right)$, para $i=1,2, \ldots, n$, em que $t_{i}$ é o instante de tempo de falha ou censura, $\delta_{i}$ é a indicadora de falha ( $\delta_{i}=1$ se em $t_{i}$ o indivíduo falhou, $\delta_{i}=0$ se em $t_{i}$ o indivíduo falhou), $\boldsymbol{x}_{i}=\left(x_{1 i}, \ldots, x_{m i}\right)^{\prime}$ é o vetor $m$-dimensional de variáveis independentes e $\boldsymbol{\theta}=\left(\theta_{1}, \ldots, \theta_{m}\right)^{\prime}$ é um vetor $m$-dimensional de parâmetros desconhecidos associado às $m$ covariáveis. Assim, a função de verossimilhança parcial para $\boldsymbol{\theta}$ é dada por

$$
L(\boldsymbol{\theta})=\prod_{i=0}^{n}\left[L_{i}(\boldsymbol{\theta})\right]^{\delta_{i}}=\prod_{i=0}^{n}\left(\frac{\exp \left\{\boldsymbol{x}_{i}^{\prime} \boldsymbol{\theta}\right\}}{\sum_{j \in R\left(t_{i}\right)} \exp \left\{\boldsymbol{x}_{j}^{\prime} \boldsymbol{\theta}\right\}}\right)^{\delta_{i}}
$$

em que $R\left(t_{i}\right)$ é o conjunto de índices dos indivíduos em risco, ou seja, que não falharam ou foram censurados no instante $t_{i}$ (Colosimo e Giolo, 2006). A Expressão A.1 supõe que não há empates nos instantes de falha observados, isto é, assume que os tempos de sobrevivência são contínuos. Para incorporar observações empatadas pode-se utilizar a aproximação para a verossimilhança parcial dada anteriormente proposta por Breslow (1972) e Peto (1972) é dada por

$$
L(\boldsymbol{\theta})=\prod_{i=0}^{n}\left[L_{i}(\boldsymbol{\theta})\right]^{\delta_{i}}=\prod_{i=0}^{n}\left(\frac{\exp \left\{\boldsymbol{s}_{i}^{\prime} \boldsymbol{\theta}\right\}}{\left[\sum_{j \in R\left(t_{i}\right)} \exp \left\{\boldsymbol{x}_{j}^{\prime} \boldsymbol{\theta}\right\}\right]^{d_{i}}}\right)^{\delta_{i}}
$$

em que $\boldsymbol{s}_{i}$ é o vetor formado pela soma das correspondentes $m$ covariáveis para os indivíduos que falham no mesmo instante $t_{i}$ e $d_{i}$ é o número de falhas neste momento.

Os valores de $\boldsymbol{\theta}$ que maximizam a verossimilhança parcial dada por (A.1) ou (A.2), estimativas de máxima verossimilhança de $\boldsymbol{\theta}$ são denotados por $\hat{\boldsymbol{\theta}}$ e são obtidos ao resolver 
o sistema de equações dado por

$$
U(\boldsymbol{\theta})=\sum_{i=0}^{n} \boldsymbol{U}_{i}(\boldsymbol{\theta})=\sum_{i=0}^{n}\left[\frac{\partial l_{i}(\boldsymbol{\theta})}{\partial \theta_{j}}\right]_{m \times 1},
$$

em que $\boldsymbol{U}(\boldsymbol{\theta})$ é o vetor score ou vetor score total, cada elemento $U_{j}$ é o score ou função score $, j=1,2, \ldots, m$, e $l_{i}(\boldsymbol{\theta})=\log \left[L_{i}(\boldsymbol{\theta})\right]$. Utilizando-se a teoria de processos de contagem e martingais (Gill, 1984), pode ser mostrado que $\boldsymbol{U}(\boldsymbol{\theta})$ é assintoticamente normal com média zero e matriz de variância e covariância igual a

$$
\mathcal{I}(\boldsymbol{\theta})=\sum_{i=0}^{n} \mathcal{I}_{i}(\boldsymbol{\theta})=\sum_{i=0}^{n}\left[E\left(-\frac{\partial^{2} l_{i}(\boldsymbol{\theta})}{\partial \theta_{j} \partial \theta_{l}}\right)\right]_{m \times m}
$$

matriz de informação de Fisher ou matriz de informação esperada. Por conveniência, diz-se que $U(\boldsymbol{\theta})$ é assintoticamente normal com média zero e matriz de covariância $\mathcal{I}(\boldsymbol{\theta})$, quando, na verdade, a versão padronizada $n^{-1 / 2} \mathcal{I}(\boldsymbol{\theta})$ é que converge para Normal.

Sob certas condições de regularidade (Kalbfleisch e Prentice, 2011), $\hat{\boldsymbol{\theta}}$ é um estimador consistente para $\boldsymbol{\theta}$, e $n^{-1 / 2} I(\hat{\boldsymbol{\theta}})$ é um estimador consistente para $n^{-1 / 2} \mathcal{I}(\boldsymbol{\theta})$, em que $I(\hat{\boldsymbol{\theta}})$ é a matriz de informação observada, dada por

$$
\boldsymbol{I}(\boldsymbol{\theta})=\sum_{i=0}^{n} \boldsymbol{I}_{i}(\boldsymbol{\theta})=\sum_{i=0}^{n}\left(-\frac{\partial^{2} l_{i}(\boldsymbol{\theta})}{\partial \theta_{j} \partial \theta_{l}}\right)_{m \times m} .
$$

Para definição dos testes, suponha o caso geral em que se queira testar as hipóteses $H_{0}: \boldsymbol{\theta}=\boldsymbol{\theta}_{0}$ versus $H_{1}: \boldsymbol{\theta} \neq \boldsymbol{\theta}_{0}$. Para aplicação do MPMIM supõe-se $\boldsymbol{\theta}_{0}=\mathbf{0}$; assim, verificase a cada ponto (ou vetor pontos) de corte, $c$, testado se a variável categorizada a partir dele tem influência significativa nos tempos de sobrevivência dos indivíduos.

\section{Teste Wald.}

O teste Wald envolve a distribuição assintótica do estimador de máxima verossimilhança (EMV) de $\boldsymbol{\theta}, \hat{\boldsymbol{\theta}}$. Se $L(\boldsymbol{\theta})$ é três vezes diferenciável e condições razoáveis de regularidade sobre a terceira derivada são satisfeitas, então pode ser demonstrado que, para uma amostra suficientemente grande, $\hat{\boldsymbol{\theta}}$ é a única solução para $U(\boldsymbol{\theta})=\mathbf{0}$ e sua distribuição assintótica é uma normal multivariada com média $\boldsymbol{\theta}$ e matriz de covariância $\mathcal{I}^{-1}(\boldsymbol{\theta})$, ou mais corretamente, sua forma padronizada $\sqrt{n}(\hat{\boldsymbol{\theta}}-\boldsymbol{\theta})$ que converge para uma normal (Kalbfleisch e Prentice, 2011). A partir desses resultados é possível fazer inferência sobre $\boldsymbol{\theta}$ e fazer estimação intervalar. A estatística de teste Wald é igual a

$$
W=\left(\hat{\boldsymbol{\theta}}-\boldsymbol{\theta}_{0}\right)^{\prime} \mathcal{I}\left(\boldsymbol{\theta}_{0}\right)\left(\hat{\boldsymbol{\theta}}-\boldsymbol{\theta}_{0}\right)
$$

e é assintoticamente distribuída por $\chi^{2}$ com $m$ graus de liberdade. Por ser um estimador consistente, a matriz de informação esperada (A.4) da Equação A.6 pode ser substituída 
pela matriz de informação observada (A.5); a substituição é necessária pela impossibilidade do cálculo de (A.4) devido a censura. Segue deste fato que uma estatística assintoticamente equivalente a A.6 e que deve ser usada para cálculo é dada por

$$
W=\left(\hat{\boldsymbol{\theta}}-\boldsymbol{\theta}_{0}\right)^{\prime} \boldsymbol{I}\left(\boldsymbol{\theta}_{0}\right)\left(\hat{\boldsymbol{\theta}}-\boldsymbol{\theta}_{0}\right) .
$$

O valor de $W$ é então comparado ao valor encontrado na tabela da distribuição de probabilidade qui-quadrado com $m$ graus de liberdade. Dado um nível de significância $\alpha$ (geralmente, 0.05$)$, se $P\left(\chi^{2}>W\right) \leq \alpha$ rejeita-se $H_{0}$, ou seja, valores altos de $W$ indicam rejeição da hipótese nula.

\section{Teste Score.}

Sabendo que, sob certas condições de regularidade, $\boldsymbol{U}(\boldsymbol{\theta})$ segue uma distribuição $N\left(0, \boldsymbol{I}\left(\boldsymbol{\theta}_{0}\right)\right)$ para amostras grandes, seja $\mathcal{I}\left(\boldsymbol{\theta}_{0}\right)$ uma matriz não singular. Então

$$
S=\boldsymbol{U}^{\prime}\left(\boldsymbol{\theta}_{0}\right)^{\prime} \mathcal{I}^{-1}\left(\boldsymbol{\theta}_{0}\right) \boldsymbol{U}\left(\boldsymbol{\theta}_{0}\right)
$$

possui distribuição assintótica $\chi^{2}$ com $m$ graus de liberdade (Lawless, 2011). Como no caso do teste Wald, sob $H_{0}, \mathcal{I}(\hat{\boldsymbol{\theta}})$ pode ser substituído por seu estimador consistente $\mathcal{I}\left(\boldsymbol{\theta}_{0}\right)$; além disso, devido à presença de censura, $\mathcal{I}(\cdot)$ não pode ser avaliada. Conforme discutido anteriormente, utilizando $\boldsymbol{I}(\cdot)$ em seu lugar, define-se a estatística de teste Score, dada por

$$
S=\boldsymbol{U}^{\prime}\left(\boldsymbol{\theta}_{0}\right)^{\prime} \boldsymbol{I}^{-1}\left(\boldsymbol{\theta}_{0}\right) \boldsymbol{U}\left(\boldsymbol{\theta}_{0}\right)
$$

O valor encontrado para $S$ é comparado ao valor encontrado na tabela da qui-quadrado com $m$ graus de liberdade. Dado um nível de significância $\alpha$, caso $P\left(\chi^{2}>S\right) \leq \alpha$ rejeitase $H_{0}$. Valores altos de $S$ indicam rejeição da hipótese nula. Note que, a estatística dada pela expressão A.9 tem a propriedade de não carecer da estimação de $\boldsymbol{\theta}$ para o seu cálculo (Lawless, 2011).

\section{Teste da razão de verossimilhança.}

A terceira estatística proposta é chamada de teste da razão de verossimilhança (TRV) (Lawless, 2011). Sob $H_{0}: \boldsymbol{\theta}=\boldsymbol{\theta}_{0}$, a distribuição assintótica da estatística

$$
T R V\left(\boldsymbol{\theta}_{0}\right)=-2 \log \left[\frac{L\left(\boldsymbol{\theta}_{0}\right)}{L(\hat{\boldsymbol{\theta}})}\right]=2 l(\hat{\boldsymbol{\theta}})-2 l\left(\boldsymbol{\theta}_{0}\right)
$$

é uma qui-quadrado com $m$ graus de liberdade. A hipótese nula é rejeitada para valores altos do TRV. 


\section{Apêndice B}

\section{Outras definições}

\section{Eficiência relativa assintótica}

Para definição da eficiência relativa assintótica (ARE, do inglês, Asymptotic Relative Efficiency), suponha $\hat{\beta}_{\text {cont }}$ o parâmetro de regressão estimado associado à variável contínua $X$ e $\hat{\beta}_{\text {cat }}$ o parâmetro de regressão estimado associado à variável categorizada a partir de $X$, então a eficiência de $\hat{\beta}_{\text {cat }}$ com respeito a $\hat{\beta}_{\text {cont }}$ é dada por

$$
A R E=\frac{V_{\text {cont }}}{V_{\text {cat }}}
$$

em que $V_{\text {cont }}$ é a variância assintótica esperada de $\hat{\beta}_{\text {cont }}$ e $V_{\text {cat }}$ é a variância assintótica esperada de $\hat{\beta}_{\text {cat }}$ (Dasgupta, 1998). Lembre que, para o modelo de Cox, os parâmetros estimados são obtidos ao maximizar a verossimilhança parcial. Os valores de ARE pertencem ao intervalo $[0, \infty)$, em que ARE $>1$ indica que $\hat{\beta}_{\text {cat }}$ é mais eficiente do que $\hat{\beta}_{\text {cont }}$.

\section{Uso da fórmula alternativa para o método das diferenças das veros- similhanças}

Suponha dois vetores de pontos de corte $\boldsymbol{c}_{1}$ e $\boldsymbol{c}_{2}$ de tal modo que $l_{\text {cat }}^{c_{1}}\left(\boldsymbol{\beta}^{*}\right)<l_{\text {cont }}(\boldsymbol{\beta})$ e $l_{\text {cat }}^{c_{2}}\left(\boldsymbol{\beta}^{*}\right)>l_{\text {cont }}(\boldsymbol{\beta})$. Segue que,

$$
\begin{gathered}
\Delta^{c_{1}}=l_{\text {cat }}^{c_{1}}\left(\boldsymbol{\beta}^{*}\right)-l_{\text {cont }}(\boldsymbol{\beta}) \geq 0 \mathrm{e} \\
\Delta^{c_{2}}=l_{\text {cat }}^{c_{2}}\left(\boldsymbol{\beta}^{*}\right)-l_{\text {cont }}(\boldsymbol{\beta}) \leq 0 .
\end{gathered}
$$

Identificar qual das diferenças $L_{c a t}^{c_{1}}-L_{\text {cont }}$ e $L_{c a t}^{c_{1}}-L_{\text {cont }}$, em módulo, é menor a partir da comparação entre os $\Delta^{c_{1}}$ e $\Delta^{c_{2}}$ é inviável por conta da natureza da função logarítmica, que decresce rapidamente para valores que se aproximam de zero. Em outras palavras, dados dois vetores de pontos de corte $c_{1}$ e $c_{2}$, de modo que $L_{c a t}^{c_{1}}-L_{c o n t}=-\left(L_{c a t}^{c_{2}}-L_{\text {cont }}\right)$, é possível que as diferenças em módulo do logaritmos das verossimilhanças sejam distintas, ou seja, $\left|l_{\text {cat }}^{c_{1}}-l_{\text {cont }}\right| \neq\left|l_{\text {cat }}^{c_{2}}-l_{\text {cont }}\right|$. 


\section{Ponte Browniana}

Uma ponte Browniana $W^{0}(u)$ é um processo estocástico Gaussiano com $u \in[0,1]$ definida como

$$
W^{0}(u)=W(u)-u W(1), 0 \leq u \leq 1,
$$

em que $\{W(u), u \geq 0\}$ é um movimento Browniano. Esse processo é tal que $E\left(W^{0}\right)=0$ e $\operatorname{Cov}\left(W^{0}(s), W^{0}(u)\right)=s(1-u)$ para $s<u$ e $s, u \in[0,1)$.

\section{Medidas de comparação}

Após o ajuste de modelos, duas estatísticas frequentemente usadas a fim de comparar os modelos são a variação explicada $R^{2}$ e o AIC (Akaike Information Criterion) definidos por

$$
\begin{aligned}
R^{2} & =1-\exp \left(-\frac{\tilde{\Gamma}}{n}\right) \quad \mathrm{e} \\
A I C & =2 k-2 \log (\hat{L}),
\end{aligned}
$$

em que $\tilde{\Gamma}$ é o TRV definido em (A.10), $n$ é o tamanho da amostra em que o teste foi aplicado, $k$ é número de variáveis no modelo e $\hat{L}$ é a verossimilhança parcial maximizada pelo parâmetro estimado (Heller e Simonoff, 1992). O $R^{2}$ é a porcentagem da variação da variável resposta explicada pela relação com uma ou mais variáveis preditoras. Quanto maior o valor de $R^{2}$, melhor o modelo ajusta os dados. O $A I C$ é uma medida de qualidade relativa de um modelo. Dado um conjunto de possíveis modelos, $A I C$ seleciona àquele que retorna a menor perda de informação relativa aos outros. Quanto menor o valor de $A I C$, melhor o modelo ajusta os dados. 


\section{Apêndice C}

\section{Tabelas para os resultados dos métodos de corre- ção e validação}

Tabela C.1: Método de validação boot no caso de dicotomia para o método MDV- caso 1

Tabela C.2: Método de validação boot no caso de dicotomia para o método MPMIN- caso 1

Tabela C.3: Método de correção $c v$ no caso de dicotomia para o método MDV- caso 1

Tabela C.4: Método de correção $c v$ no caso de dicotomia para o método MPMIN- caso 1

Tabela C.5: Método de validação boot no caso de tricotomia para o método MDV- caso 1

Tabela C.6: Método de validação boot no caso de tricotomia para o método MPMIN- caso 1

Tabela C.7: Método de correção $c v$ no caso de tricotomia para o método MDV- caso 1

Tabela C.8: Método de correção $c v$ no caso de tricotomia para o método MPMIN- caso 1

Tabela C.9: Método de correção $f c$ no caso de dicotomia para o cenário univariado- caso 1

Tabela C.10: Método de validação boot no caso de dicotomia para o método MDV- caso 2

Tabela C.11: Método de validação boot no caso de dicotomia para o método MPMIN-caso 2

Tabela C.12: Método de correção $c v$ no caso de dicotomia para o método MDV- caso 2

Tabela C.13: Método de correção $c v$ no caso de dicotomia para o método MPMIN- caso 2

Tabela C.14: Método de validação boot no caso de tricotomia para o método MDV- caso 2

Tabela C.15: Método de validação boot no caso de tricotomia para o método MPMIN-caso 2

Tabela C.16: Método de correção $c v$ no caso de tricotomia para o método MDV- caso 2

Tabela C.17: Método de correção $c v$ no caso de tricotomia para o método MPMIN- caso 2

Tabela C.18: Método de correção $f c$ no caso de dicotomia para o cenário univariado- caso 2 
Tabela C.1: Estatisticas obtidas a partir de 2000 amostras bootstrap para os pontos de corte $e$ riscos relativos, ci e rri, no caso de dicotomia, em que $i=1$,2. Os indices 1 e 2 referem-se aos métodos MDV1 e MDV2, respectivamente. Além disso, o procedimento foi realizada para cada um dos 4 cenários avaliados.

\begin{tabular}{|c|c|c|c|c|c|c|}
\hline Cenário & Medida & Média & Média aparada & Moda & Mediana & Desvio padrão \\
\hline \multirow{4}{*}{ M1 } & $\mathrm{c} 1$ & 37.22 & 36.30 & 40.00 & 36.00 & 8.10 \\
\hline & c2 & 35.35 & 34.48 & 35.00 & 34.00 & 7.64 \\
\hline & rr1 & 0.80 & 0.79 & 0.78 & 0.78 & 0.09 \\
\hline & $\operatorname{rr} 2$ & 0.79 & 0.78 & 0.76 & 0.76 & 0.08 \\
\hline \multirow{4}{*}{ M2 } & $\mathrm{c} 1$ & 33.04 & 33.32 & 36.00 & 34.00 & 3.98 \\
\hline & $\mathrm{c} 2$ & 31.64 & 31.63 & 34.00 & 32.00 & 3.71 \\
\hline & rr1 & 0.62 & 0.62 & 0.60 & 0.62 & 0.05 \\
\hline & $\mathrm{rr} 2$ & 0.60 & 0.60 & 0.59 & 0.60 & 0.05 \\
\hline \multirow{4}{*}{ M3 } & c1 & 32.61 & 32.35 & 35.00 & 33.00 & 4.47 \\
\hline & $c 2$ & 31.41 & 31.06 & 31.00 & 31.00 & 4.06 \\
\hline & $\operatorname{rr} 1$ & 0.61 & 0.61 & 0.61 & 0.61 & 0.05 \\
\hline & $\operatorname{rr} 2$ & 0.60 & 0.60 & 0.58 & 0.60 & 0.05 \\
\hline \multirow{4}{*}{ Uni } & $\mathrm{c} 1$ & 32.96 & 33.05 & 35.00 & 33.00 & 4.53 \\
\hline & $\mathrm{c} 2$ & 32.02 & 31.74 & 31.00 & 32.00 & 4.22 \\
\hline & rr1 & 0.59 & 0.59 & 0.59 & 0.59 & 0.04 \\
\hline & $\operatorname{rr} 2$ & 0.59 & 0.59 & 0.60 & 0.59 & 0.04 \\
\hline
\end{tabular}

Tabela C.2: Estatísticas obtidas a partir de 2000 amostras bootstrap para os pontos de corte $e$ riscos relativos, ci e rri, no caso de dicotomia, em que $i=1$,2,3. Os indices 1, 2 e 3 referem-se aos métodos MPMIN1, MPMIN2 e MPMIN3, respectivamente. Além disso, o procedimento foi realizada para cada um dos 4 cenários avaliados. Todas as estatísticas para os valores-p foram inferiores a 0.01 e, portanto, foram omitidas da tabela.

\begin{tabular}{ccccccc} 
Cenário & Medida & Média & Média aparada & Moda & Mediana & Desvio padrão \\
\hline \multirow{3}{*}{ M1 } & c1 & 30.51 & 29.33 & 29.00 & 29.5 & 4.89 \\
& c2 & 30.42 & 29.25 & 29.00 & 29.0 & 4.87 \\
& rr1 & 0.70 & 0.70 & 0.69 & 0.70 & 0.06 \\
& rr2 & 0.70 & 0.70 & 0.69 & 0.70 & 0.06 \\
M2 & c1 & 28.92 & 28.49 & 29.00 & 29.00 & 2.46 \\
& c2 & 28.46 & 28.16 & 29.00 & 29.00 & 2.38 \\
& rr1 & 0.55 & 0.55 & 0.55 & 0.55 & 0.04 \\
\multirow{4}{*}{ M3 } & rr2 & 0.55 & 0.55 & 0.55 & 0.55 & 0.04 \\
& $\mathrm{c} 1$ & 29.87 & 29.69 & 29.00 & 30.00 & 2.84 \\
& $\mathrm{c} 2$ & 29.41 & 28.89 & 29.00 & 29.00 & 2.60 \\
& $\mathrm{rr} 1$ & 0.56 & 0.56 & 0.57 & 0.56 & 0.04 \\
& $\mathrm{rr} 2$ & 0.56 & 0.56 & 0.57 & 0.56 & 0.04 \\
& $\mathrm{c} 1$ & 30.36 & 30.04 & 30.00 & 30.00 & 3.10 \\
& $\mathrm{c} 2$ & 29.44 & 29.22 & 30.00 & 29.00 & 2.62 \\
& $\mathrm{c} 3$ & 29.43 & 29.22 & 30.00 & 29.00 & 2.64 \\
& $\mathrm{rr} 1$ & 0.56 & 0.56 & 0.55 ou 0.57 & 0.56 & 0.04 \\
& $\mathrm{rr} 2$ & 0.56 & 0.56 & 0.57 & 0.56 & 0.04 \\
& $\mathrm{rr} 3$ & 0.56 & 0.56 & 0.57 & 0.56 & 0.04
\end{tabular}


Tabela C.3: Estatísticas obtidas a partir de 1000 repetições do método de validação cruzada para obtenção dos riscos relativos e valores-p, ci e pi, no caso de dicotomia, em que $i=1$ e 2. Os índices 1 e 2 referem-se aos métodos MDV1 e MDV2, respectivamente. Além disso, o procedimento foi realizada para cada um dos 4 cenários avaliados.

\begin{tabular}{ccccccc} 
Cenário & Medida & Média & Média aparada & Moda & Mediana & Desvio padrão \\
\hline \multirow{2}{*}{ M1 } & rr1 & 0.81 & 0.81 & 0.81 & 0.81 & 0.04 \\
& rr2 & 0.81 & 0.81 & 0.81 & 0.81 & 0.04 \\
M2 & rr1 & 0.61 & 0.61 & 0.61 & 0.61 & 0.03 \\
& rr2 & 0.60 & 0.60 & 0.60 & 0.60 & 0.03 \\
\multirow{2}{*}{ M3 } & rr1 & 0.61 & 0.61 & 0.61 & 0.61 & 0.02 \\
& rr2 & 0.62 & 0.62 & 0.62 & 0.62 & 0.01 \\
\multirow{2}{*}{ Uni } & rr1 & 0.60 & 0.60 & 0.61 & 0.60 & 0.02 \\
& rr2 & 0.60 & 0.60 & 0.59 & 0.60 & 0.02 \\
\hline
\end{tabular}

Tabela C.4: Estatisticas obtidas a partir de 1000 repetições do método de validação cruzada para estimação dos riscos relativos e valores-p, rri e pi, no caso de dicotomia, em que $i=1$,2,3. Os índices 1, 2 e 3 referem-se aos métodos MPMIN1, MPMIN2 e MPMIN3, respectivamente. Além disso, o procedimento foi realizada para cada um dos 4 cenários avaliados. Todas as estatísticas para os valores-p foram inferiores a 0.01 e, portanto, foram omitidas da tabela, exceto para o cenário M1.

\begin{tabular}{ccccccc} 
Cenário & Medida & Média & Média aparada & Moda & Mediana & Desvio padrão \\
\hline \multirow{3}{*}{ M1 } & rr1 & 0.81 & 0.80 & 0.79 & 0.79 & 0.05 \\
& rr2 & 0.80 & 0.80 & 0.79 & 0.79 & 0.05 \\
& $\mathrm{p} 1$ & $<0.01$ & $<0.01$ & $<0.01$ & $<0.01$ & $<0.01$ \\
\multirow{3}{*}{ M2 } & $\mathrm{p} 2$ & 0.05 & 0.02 & 0.01 & 0.01 & 0.14 \\
& $\mathrm{rr} 1$ & 0.60 & 0.60 & 0.60 & 0.60 & 0.02 \\
$\mathrm{~N} 3$ & $\mathrm{rr} 2$ & 0.60 & 0.60 & 0.60 & 0.60 & 0.02 \\
& $\mathrm{rr} 1$ & 0.62 & 0.62 & 0.61 & 0.62 & 0.03 \\
\multirow{3}{*}{ Uni } & $\mathrm{rr} 2$ & 0.62 & 0.62 & 0.61 & 0.61 & 0.02 \\
& $\mathrm{rr} 1$ & 0.60 & 0.60 & 0.61 & 0.60 & 0.02 \\
& $\mathrm{rr} 2$ & 0.60 & 0.60 & 0.58 & 0.59 & 0.02 \\
\hline
\end{tabular}


Tabela C.5: Estatisticas obtidas a partir de 2000 amostras bootstrap para seleção dos pontos de corte e estimação dos riscos relativos, cij e rrij, no caso de tricotomia, em que o ponto de corte $i=1$, 2 é estimado pelo método $j=1$,2. $O$ indice $i=1$ indica o limite entre os grupos 1 e 2 da FE e o indice $i=2$ indica o limite entre os grupos 2 e 3 da FE. O indice $j$ refere-se ao método $M D V j$. Além disso, o procedimento foi realizada para cada um dos 4 cenários avaliados.

\begin{tabular}{|c|c|c|c|c|c|c|}
\hline Cenário & Medida & Média & Média aparada & Moda & Mediana & Desvio padrão \\
\hline \multirow{8}{*}{ M1 } & $\mathrm{c} 11$ & 35.55 & 34.78 & 34.00 & 35.00 & 6.87 \\
\hline & $c 21$ & 50.90 & 51.45 & 57.00 & 52.00 & 6.83 \\
\hline & c12 & 35.10 & 34.28 & 26.00 & 35.00 & 6.73 \\
\hline & $\mathrm{c} 22$ & 50.40 & 50.92 & 54.00 & 52.00 & 7.01 \\
\hline & $\operatorname{rr} 11$ & 0.82 & 0.81 & 0.80 & 0.81 & 0.10 \\
\hline & $\operatorname{rr} 21$ & 0.78 & 0.78 & 0.74 & 0.77 & 0.12 \\
\hline & $\operatorname{rr} 12$ & 0.81 & 0.80 & 0.77 & 0.80 & 0.10 \\
\hline & rr22 & 0.78 & 0.77 & 0.74 & 0.77 & 0.12 \\
\hline \multirow{8}{*}{ M2 } & c11 & 32.59 & 32.42 & 36.00 & 33.00 & 4.10 \\
\hline & $\mathrm{c} 21$ & 48.69 & 49.15 & 45.00 & 49.00 & 6.49 \\
\hline & c12 & 31.88 & 31.54 & 34.00 & 32.00 & 4.00 \\
\hline & $\mathrm{c} 22$ & 48.74 & 48.99 & 45.00 & 49.00 & 6.63 \\
\hline & $\operatorname{rr} 11$ & 0.64 & 0.64 & 0.63 & 0.64 & 0.06 \\
\hline & rr21 & 0.59 & 0.59 & 0.55 & 0.58 & 0.09 \\
\hline & $\operatorname{rr} 12$ & 0.63 & 0.63 & 0.62 & 0.63 & 0.06 \\
\hline & rr22 & 0.58 & 0.58 & 0.56 & 0.58 & 0.08 \\
\hline \multirow{8}{*}{ M3 } & c11 & 31.97 & 31.67 & 34.00 & 32.00 & 4.61 \\
\hline & $c 21$ & 48.32 & 48.89 & 54.00 & 49.00 & 6.64 \\
\hline & c12 & 31.43 & 31.16 & 26.00 & 32.00 & 4.46 \\
\hline & $\mathrm{c} 22$ & 48.23 & 48.76 & 54.00 & 49.00 & 6.74 \\
\hline & $\operatorname{rr} 11$ & 0.65 & 0.65 & 0.63 & 0.65 & 0.07 \\
\hline & rr21 & 0.58 & 0.57 & 0.55 & 0.57 & 0.08 \\
\hline & $\operatorname{rr} 12$ & 0.65 & 0.65 & 0.63 & 0.64 & 0.07 \\
\hline & $\operatorname{rr} 22$ & 0.57 & 0.57 & 0.55 ou 0.57 & 0.57 & 0.08 \\
\hline \multirow{8}{*}{ Uni } & c11 & 33.35 & 33.41 & 32.00 & 33.00 & 4.31 \\
\hline & $\mathrm{c} 21$ & 48.36 & 48.63 & 55.00 & 49.00 & 6.52 \\
\hline & c12 & 32.72 & 32.43 & 32.00 & 33.00 & 4.33 \\
\hline & c22 & 48.34 & 48.84 & 45 ou 56.00 & 49.00 & 6.59 \\
\hline & $\operatorname{rr} 11$ & 0.66 & 0.66 & 0.66 & 0.66 & 0.07 \\
\hline & $\operatorname{rr} 21$ & 0.62 & 0.62 & 0.61 & 0.61 & 0.08 \\
\hline & $\operatorname{rr} 12$ & 0.65 & 0.65 & 0.64 & 0.65 & 0.07 \\
\hline & rr22 & 0.61 & 0.61 & 0.61 & 0.61 & 0.07 \\
\hline
\end{tabular}


Tabela C.6: Estatísticas obtidas a partir de 2000 amostras bootstrap para seleção dos pontos de corte e estimação dos riscos relativos, cij e rrij, no caso de tricotomia, em que o ponto de corte $i=1$,2 é estimado pelo método $j=1$,2,3. $O$ indice $i=1$ indica o limite entre os grupos 1 e 2 da FE e $o$ indice $i=2$ indica o limite entre os grupos 2 e 3 da FE. $O$ indice $j$ refere-se ao método MPMINj. Além disso, o procedimento foi realizada para cada um dos 4 cenários avaliados. Todas as estatisticas para os valores-p foram inferiores a 0.01 e, portanto, foram omitidas da tabela.

\begin{tabular}{|c|c|c|c|c|c|c|}
\hline Cenário & Medida & Média & Média aparada & Moda & Mediana & Desvio padrão \\
\hline \multirow{8}{*}{ M1 } & $\mathrm{c} 11$ & 29.16 & 28.24 & 29.00 & 29.00 & 3.88 \\
\hline & $c 21$ & 45.00 & 45.08 & 40.00 & 43.00 & 9.26 \\
\hline & $\mathrm{c} 12$ & 28.91 & 28.15 & 29.00 & 29.00 & 3.64 \\
\hline & $\mathrm{c} 22$ & 45.09 & 45.03 & 40.00 & 43.00 & 9.36 \\
\hline & $\operatorname{rr} 11$ & 0.73 & 0.72 & 0.76 & 0.72 & 0.10 \\
\hline & rr21 & 0.66 & 0.65 & 0.57 ou 0.62 & 0.62 & 0.12 \\
\hline & $\operatorname{rr} 12$ & 0.73 & 0.73 & 0.76 & 0.73 & 0.11 \\
\hline & rr22 & 0.65 & 0.64 & 0.57 & 0.62 & 0.12 \\
\hline \multirow{8}{*}{ M2 } & $\mathrm{c} 11$ & 26.31 & 26.02 & 25.00 & 25.00 & 1.90 \\
\hline & $c 21$ & 37.47 & 35.76 & 30.00 & 34.00 & 9.21 \\
\hline & c12 & 26.31 & 26.10 & 25.00 & 25.00 & 1.83 \\
\hline & $\mathrm{c} 22$ & 38.06 & 36.32 & 30.00 & 35.00 & 9.14 \\
\hline & $\operatorname{rr} 11$ & 0.67 & 0.67 & 0.67 & 0.66 & 0.08 \\
\hline & rr21 & 0.48 & 0.48 & 0.46 & 0.48 & 0.05 \\
\hline & $\operatorname{rr} 12$ & 0.66 & 0.66 & 0.63 & 0.66 & 0.08 \\
\hline & rr22 & 0.48 & 0.48 & 0.46 & 0.48 & 0.05 \\
\hline \multirow{8}{*}{ M3 } & c11 & 26.81 & 26.57 & 25.00 & 25.00 & 2.10 \\
\hline & c21 & 38.07 & 36.22 & 30.00 & 35.00 & 7.79 \\
\hline & c12 & 26.99 & 26.80 & 25.00 & 27.00 & 2.03 \\
\hline & $\mathrm{c} 22$ & 38.70 & 37.57 & 35.00 & 36.00 & 7.52 \\
\hline & rr11 & 0.69 & 0.68 & 0.65 & 0.68 & 0.08 \\
\hline & rr21 & 0.49 & 0.49 & 0.48 & 0.48 & 0.05 \\
\hline & $\operatorname{rr} 12$ & 0.68 & 0.67 & 0.65 & 0.67 & 0.08 \\
\hline & rr22 & 0.49 & 0.49 & 0.48 & 0.49 & 0.05 \\
\hline \multirow{12}{*}{ Uni } & c11 & 27.55 & 27.44 & 25.00 & 28.00 & 2.03 \\
\hline & c21 & 41.02 & 40.79 & 40.00 & 40.00 & 7.82 \\
\hline & c12 & 27.15 & 27.08 & 25.00 & 27.50 & 1.95 \\
\hline & $\mathrm{c} 22$ & 39.23 & 37.45 & 40.00 & 38.00 & 7.39 \\
\hline & c13 & 27.14 & 27.08 & 25.00 & 27.00 & 1.95 \\
\hline & $c 23$ & 39.35 & 37.52 & 40.00 & 38.00 & 7.44 \\
\hline & rr11 & 0.67 & 0.67 & 0.66 & 0.67 & 0.06 \\
\hline & rr21 & 0.49 & 0.49 & 0.47 & 0.49 & 0.04 \\
\hline & rr12 & 0.69 & 0.68 & 0.70 & 0.68 & 0.07 \\
\hline & rr22 & 0.49 & 0.49 & 0.48 & 0.49 & 0.04 \\
\hline & rr13 & 0.69 & 0.68 & 0.70 & 0.68 & 0.07 \\
\hline & rr23 & 0.49 & 0.49 & 0.48 & 0.49 & 0.04 \\
\hline
\end{tabular}


Tabela C.7: Estatísticas obtidas a partir de 1000 repetições do método de validação cruzada para obtenção dos pontos de corte e estimação dos riscos relativos, cij e rrij, no caso de tricotomia, em que o ponto de corte $i=1,2$ é estimado pelo método $j=1$,2. $O$ indice $i=1$ indica o limite entre os grupos 1 e 2 da FE e o índice $i=2$ indica o limite entre os grupos 2 e 3 da FE. $O$ indice $j$ refere-se ao método $M D V j$. Além disso, o procedimento foi realizada para cada um dos 4 cenários avaliados.

\begin{tabular}{ccccccc} 
Cenário & Medida & Média & Média aparada & Moda & Mediana & Desvio padrão \\
\hline \multirow{3}{*}{ M1 } & rr11 & 0.80 & 0.80 & 0.81 & 0.80 & 0.06 \\
& $\operatorname{rr} 21$ & 0.77 & 0.77 & 0.75 & 0.76 & 0.05 \\
& $\operatorname{rr} 12$ & 0.80 & 0.79 & 0.80 & 0.79 & 0.06 \\
& $\operatorname{rr} 22$ & 0.78 & 0.78 & 0.78 & 0.78 & 0.06 \\
\multirow{3}{*}{ M2 } & $\operatorname{rr} 11$ & 0.62 & 0.62 & 0.62 & 0.62 & 0.04 \\
& $\operatorname{rr} 21$ & 0.58 & 0.58 & 0.59 & 0.59 & 0.04 \\
& $\operatorname{rr} 12$ & 0.60 & 0.60 & 0.60 & 0.60 & 0.04 \\
\multirow{3}{*}{ M3 } & $\operatorname{rr} 22$ & 0.58 & 0.58 & 0.57 & 0.58 & 0.05 \\
& $\operatorname{rr} 11$ & 0.66 & 0.66 & 0.65 & 0.66 & 0.04 \\
& $\operatorname{rr} 21$ & 0.60 & 0.60 & 0.61 & 0.60 & 0.04 \\
& $\operatorname{rr} 12$ & 0.64 & 0.64 & 0.64 & 0.64 & 0.04 \\
\multirow{3}{*}{ Uni } & $\operatorname{rr} 22$ & 0.61 & 0.61 & 0.61 & 0.61 & 0.04 \\
& $\operatorname{rr} 11$ & 0.65 & 0.65 & 0.67 & 0.65 & 0.04 \\
& $\operatorname{rr} 21$ & 0.60 & 0.60 & 0.61 & 0.61 & 0.03 \\
& $\operatorname{rr} 12$ & 0.63 & 0.63 & 0.63 & 0.63 & 0.04 \\
\hline
\end{tabular}


Tabela C.8: Estatísticas obtidas a partir de 1000 repetições do método de validação cruzada para estimação dos riscos relativos e valores-p, rrij e pj, no caso de tricotomia, em que o ponto de corte $i=1,2$ é estimado pelo método $j=1,2,3 . O$ indice $i=1$ indica o limite entre os grupos 1 e 2 da FE e o indice $i=2$ indica o limite entre os grupos 2 e 3 da FE. $O$ índice $j$ refere-se ao método MPMINj. Além disso, o procedimento foi realizada para cada um dos 4 cenários avaliados. Todas as estatísticas para os valores-p foram inferiores a $0.01 \mathrm{e}$, portanto, foram omitidas da tabela, exceto para o cenário M1.

\begin{tabular}{|c|c|c|c|c|c|c|}
\hline Cenário & Medida & Média & Média aparada & Moda & Mediana & Desvio padrão \\
\hline \multirow{6}{*}{ M1 } & $\operatorname{rr} 11$ & 0.81 & 0.80 & 0.80 & 0.80 & 0.05 \\
\hline & rr21 & 0.75 & 0.75 & 0.77 & 0.75 & 0.04 \\
\hline & $\operatorname{rr} 12$ & 0.80 & 0.79 & 0.79 & 0.79 & 0.05 \\
\hline & rr22 & 0.77 & 0.77 & 0.77 & 0.77 & 0.06 \\
\hline & p1 & 0.03 & 0.01 & 0.01 & 0.01 & 0.07 \\
\hline & p2 & 0.03 & 0.01 & 0.01 & 0.01 & 0.07 \\
\hline \multirow{4}{*}{ M2 } & $\operatorname{rr} 11$ & 0.65 & 0.65 & 0.65 & 0.65 & 0.04 \\
\hline & $\operatorname{rr} 21$ & 0.54 & 0.53 & 0.54 & 0.53 & 0.02 \\
\hline & $\operatorname{rr} 12$ & 0.61 & 0.61 & 0.62 & 0.61 & 0.03 \\
\hline & $\operatorname{rr} 22$ & 0.56 & 0.56 & 0.57 & 0.56 & 0.03 \\
\hline \multirow{4}{*}{ M3 } & $\operatorname{rr} 11$ & 0.69 & 0.69 & 0.69 & 0.69 & 0.03 \\
\hline & $\operatorname{rr} 21$ & 0.59 & 0.59 & 0.60 & 0.58 & 0.02 \\
\hline & $\operatorname{rr} 12$ & 0.66 & 0.65 & 0.66 & 0.65 & 0.03 \\
\hline & $\operatorname{rr} 22$ & 0.60 & 0.60 & 0.59 & 0.60 & 0.03 \\
\hline \multirow{6}{*}{ Uni } & $\operatorname{rr} 11$ & 0.67 & 0.67 & 0.68 & 0.67 & 0.03 \\
\hline & $\operatorname{rr} 21$ & 0.54 & 0.53 & 0.53 & 0.53 & 0.01 \\
\hline & $\operatorname{rr} 12$ & 0.62 & 0.62 & 0.62 & 0.62 & 0.03 \\
\hline & rr22 & 0.55 & 0.55 & 0.55 & 0.55 & 0.02 \\
\hline & $\operatorname{rr} 13$ & 0.62 & 0.62 & 0.62 & 0.62 & 0.03 \\
\hline & rr23 & 0.55 & 0.55 & 0.55 & 0.55 & 0.02 \\
\hline
\end{tabular}


Tabela C.9: Fatores de contração heurístico, bootstrap e validação cruzada para o cenário univariado no caso de dicotomia.

\begin{tabular}{|c|c|c|c|c|}
\hline Fator de contração & Método & $c$ & $\hat{d}$ & $\widehat{r r}$ \\
\hline \multirow{3}{*}{ Heurístico } & MDV1 & 27 & 0.9852 & 0.5768 \\
\hline & MDV2 & 28 & 0.9859 & 0.5731 \\
\hline & MPMIN & 30 & 0.9866 & 0.5786 \\
\hline \multirow{3}{*}{ Heurístico* } & MDV1 & 35 & 0.9836 & 0.6094 \\
\hline & MDV2 & 31 & 0.9854 & 0.5929 \\
\hline & MPMIN & 30 & 0.9866 & 0.5786 \\
\hline \multirow{3}{*}{ Bootstrap1 } & MPMIN1 & - & - & 0.6053 \\
\hline & MPMIN2 & - & - & 0.6042 \\
\hline & MPMIN3 & - & - & 0.6046 \\
\hline \multirow{3}{*}{ Bootstrap2 } & MPMIN1 & - & 0.9119 & 0.6031 \\
\hline & MPMIN2 & - & 0.9157 & 0.6018 \\
\hline & MPMIN3 & - & 0.9148 & 0.6021 \\
\hline \multirow{3}{*}{ Validação Cruzada } & MDV1 & 27 & 0.9549 & 0.5866 \\
\hline & MDV2 & 28 & 0.9773 & 0.5759 \\
\hline & MPMIN & 30 & 0.9676 & 0.5847 \\
\hline \multirow{3}{*}{ Validação Cruzada* } & MDV1 & 35 & 0.9664 & 0.6147 \\
\hline & MDV2 & 31 & 0.9641 & 0.5997 \\
\hline & MPMIN & 30 & 0.9676 & 0.5847 \\
\hline
\end{tabular}

\footnotetext{
${ }^{\mathrm{i}}$ Heurístico* indica o fator de contração heurístico aplicado aos pontos de corte validados pelo método boot.

ii Validação cruzada* indica o fator de contração validação cruzada aplicado aos pontos de corte validados pelo método boot.
}

Tabela C.10: Estatísticas obtidas a partir de 2000 amostras bootstrap para os pontos de corte e riscos relativos, ci e rri, no caso de dicotomia, em que $i=1$,2. Os índices 1 e 2 referem-se aos métodos MDV1 e MDV2, respectivamente. Além disso, o procedimento foi realizada para cada um dos 3 cenários avaliados.

\begin{tabular}{ccccccc} 
Cenário & Medida & Média & Média aparada & Moda & Mediana & Desvio padrão \\
\hline \multirow{4}{*}{ M1 } & c1 & 32.55 & 32.37 & 35.00 & 33.00 & 4.25 \\
& c2 & 31.31 & 31.08 & 31.00 & 31.00 & 3.93 \\
& rr1 & 0.62 & 0.62 & 0.63 & 0.62 & 0.05 \\
& rr2 & 0.61 & 0.61 & 0.61 & 0.61 & 0.05 \\
\multirow{3}{*}{ M2 } & c1 & 32.14 & 31.87 & 35.00 & 32.00 & 4.12 \\
& c2 & 30.90 & 30.74 & 31.00 & 31.00 & 3.81 \\
& rr1 & 0.63 & 0.63 & 0.62 & 0.63 & 0.05 \\
& rr2 & 0.62 & 0.62 & 0.61 & 0.62 & 0.05 \\
\multirow{3}{*}{ Uni } & c1 & 33.38 & 33.54 & 36.00 & 34.00 & 4.29 \\
& $\mathrm{c} 2$ & 31.86 & 31.56 & 31.00 & 32.00 & 4.06 \\
& rr1 & 0.63 & 0.63 & 0.62 & 0.63 & 0.05 \\
& rr2 & 0.62 & 0.62 & 0.62 & 0.62 & 0.05 \\
\hline
\end{tabular}


Tabela C.11: Estatísticas obtidas a partir de 2000 amostras bootstrap para os pontos de corte $e$ riscos relativos, ci e rri, no caso de dicotomia, em que $i=1$,2,3. Os indices 1, 2 e 3 referem-se aos métodos MPMIN1, MPMIN2 e MPMIN3, respectivamente. Além disso, o procedimento foi realizada para cada um dos 3 cenários avaliados. Todas as estatisticas para os valores-p foram inferiores a 0.01 e, portanto, foram omitidas da tabela.

\begin{tabular}{ccccccc} 
Cenário & Medida & Média & Média aparada & Moda & Mediana & Desvio padrão \\
\hline \multirow{3}{*}{ M1 } & $\mathrm{c} 1$ & 29.27 & 28.60 & 29.0 & 29.00 & 2.74 \\
& $\mathrm{c} 2$ & 28.65 & 28.26 & 29.0 & 29.00 & 2.48 \\
& $\mathrm{rr} 1$ & 0.56 & 0.56 & 0.55 & 0.56 & 0.04 \\
& $\mathrm{rr} 2$ & 0.56 & 0.56 & 0.56 & 0.56 & 0.04 \\
\multirow{3}{*}{ M2 } & $\mathrm{c} 1$ & 28.81 & 28.19 & 29.00 & 29.00 & 2.72 \\
& $\mathrm{c} 2$ & 28.25 & 27.70 & 29.00 & 29.00 & 2.51 \\
& $\mathrm{rr} 1$ & 0.58 & 0.57 & 0.58 & 0.58 & 0.04 \\
& $\mathrm{rr} 2$ & 0.57 & 0.57 & 0.58 & 0.57 & 0.04 \\
& $\mathrm{c} 1$ & 28.97 & 28.43 & 30.00 & 29.00 & 2.46 \\
\multirow{3}{*}{ Uni } & $\mathrm{c} 2$ & 28.39 & 28.07 & 28.00 & 28.00 & 2.23 \\
& $\mathrm{c} 3$ & 28.36 & 28.06 & 28.00 & 28.00 & 2.24 \\
& $\mathrm{rr} 1$ & 0.57 & 0.57 & 0.57 & 0.57 & 0.04 \\
& $\mathrm{rr} 2$ & 0.57 & 0.57 & 0.57 & 0.57 ou 0.58 & 0.04 \\
& $\mathrm{rr} 3$ & 0.57 & 0.57 & 0.57 & 0.57 & 0.04 \\
\hline
\end{tabular}

Tabela C.12: Estatisticas obtidas a partir de 1000 repetições do método de validação cruzada para obtenção dos riscos relativos e valores-p, ci e pi, no caso de dicotomia, em que $i=1$ e 2. Os índices 1 e 2 referem-se aos métodos MDV1 e MDV2, respectivamente. Além disso, o procedimento foi realizada para cada um dos 3 cenários avaliados.

\begin{tabular}{ccccccc} 
Cenário & Medida & Média & Média aparada & Moda & Mediana & Desvio padrão \\
\hline \multirow{2}{*}{ M1 } & rr1 & 0.65 & 0.65 & 0.66 & 0.65 & 0.01 \\
& rr2 & 0.57 & 0.57 & 0.57 & 0.57 & $<0.01$ \\
\multirow{2}{*}{ M2 } & rr1 & 0.63 & 0.63 & 0.62 & 0.62 & 0.03 \\
& rr2 & 0.62 & 0.62 & 0.62 & 0.62 & 0.02 \\
\multirow{2}{*}{ Uni } & rr1 & 0.63 & 0.63 & 0.63 & 0.63 & 0.03 \\
& rr2 & 0.62 & 0.62 & 0.61 & 0.62 & 0.02 \\
\hline
\end{tabular}

Tabela C.13: Estatísticas obtidas a partir de 1000 repetições do método de validação cruzada para estimação dos riscos relativos e valores- , rri e pi, no caso de dicotomia, em que $i=1$,2,3. Os índices 1, 2 e 3 referem-se aos métodos MPMIN1, MPMIN2 e MPMIN3, respectivamente. Além disso, o procedimento foi realizada para cada um dos 3 cenários avaliados. Todas as estatísticas para os valores-p foram inferiores a 0.01 e, portanto, foram omitidas da tabela, exceto para o cenário M1.

\begin{tabular}{ccccccc} 
Cenário & Medida & Média & Média aparada & Moda & Mediana & Desvio padrão \\
\hline \multirow{2}{*}{ M1 } & rr1 & 0.62 & 0.61 & 0.61 & 0.61 & 0.02 \\
& rr2 & 0.61 & 0.61 & 0.60 & 0.61 & 0.02 \\
\multirow{2}{*}{ M2 } & rr1 & 0.62 & 0.62 & 0.62 & 0.62 & 0.02 \\
& rr2 & 0.62 & 0.61 & 0.60 & 0.61 & 0.02 \\
\multirow{2}{*}{ Uni } & rr1 & 0.61 & 0.61 & 0.60 & 0.61 & 0.02 \\
& rr2 & 0.61 & 0.61 & 0.60 & 0.61 & 0.02 \\
& rr3 & 0.61 & 0.61 & 0.60 & 0.61 & 0.02 \\
\hline
\end{tabular}


Tabela C.14: Estatísticas obtidas a partir de 2000 amostras bootstrap para seleção dos pontos de corte e estimação dos riscos relativos, cij e rrij, no caso de tricotomia, em que o ponto de corte $i=1,2$ é estimado pelo método $j=1,2$. O indice $i=1$ indica o limite entre os grupos 1 e 2 da FE e o indice $i=2$ indica o limite entre os grupos 2 e 3 da FE. $O$ indice j refere-se ao método MDVj. Além disso, o procedimento foi realizada para cada um dos 3 cenários avaliados.

\begin{tabular}{|c|c|c|c|c|c|c|}
\hline Cenário & Medida & Média & Média aparada & Moda & Mediana & Desvio padrão \\
\hline \multirow{8}{*}{ M1 } & c11 & 32.41 & 32.02 & 32.00 & 32.00 & 4.15 \\
\hline & c21 & 48.07 & 48.60 & 45.00 & 48.00 & 6.87 \\
\hline & c12 & 31.84 & 31.56 & 31.00 & 32.00 & 4.14 \\
\hline & c22 & 47.99 & 48.48 & 45.00 & 48.00 & 6.89 \\
\hline & $\operatorname{rr} 11$ & 0.67 & 0.67 & 0.69 & 0.67 & 0.07 \\
\hline & $\operatorname{rr} 21$ & 0.62 & 0.62 & 0.59 & 0.61 & 0.08 \\
\hline & $\operatorname{rr} 12$ & 0.66 & 0.66 & 0.64 & 0.66 & 0.06 \\
\hline & $\operatorname{rr} 22$ & 0.62 & 0.61 & 0.60 & 0.61 & 0.07 \\
\hline \multirow{8}{*}{ M2 } & c11 & 31.72 & 31.55 & 32.00 & 32.00 & 4.01 \\
\hline & c21 & 48.08 & 48.42 & 45.00 & 48.50 & 6.92 \\
\hline & $\mathrm{c} 12$ & 31.15 & 30.85 & 31.00 & 31.00 & 3.86 \\
\hline & $\mathrm{c} 22$ & 47.52 & 47.96 & 45.00 & 48.00 & 7.01 \\
\hline & $\operatorname{rr} 11$ & 0.67 & 0.67 & 0.66 & 0.66 & 0.06 \\
\hline & $\operatorname{rr} 21$ & 0.60 & 0.59 & 0.58 & 0.59 & 0.07 \\
\hline & $\operatorname{rr} 12$ & 0.66 & 0.66 & 0.64 & 0.66 & 0.06 \\
\hline & $\operatorname{rr} 22$ & 0.59 & 0.59 & 0.60 & 0.59 & 0.07 \\
\hline \multirow{8}{*}{ Uni } & c11 & 33.35 & 33.41 & 32.00 & 33.00 & 4.31 \\
\hline & $\mathrm{c} 21$ & 48.36 & 48.63 & 55.00 & 49.00 & 6.52 \\
\hline & $\mathrm{c} 12$ & 32.72 & 32.43 & 32.00 & 33.00 & 4.33 \\
\hline & c22 & 48.34 & 48.84 & 45 ou 56.00 & 49.00 & 6.59 \\
\hline & $\operatorname{rr} 11$ & 0.66 & 0.66 & 0.66 & 0.66 & 0.07 \\
\hline & $\operatorname{rr} 21$ & 0.62 & 0.62 & 0.57 ou 0.61 & 0.61 & 0.08 \\
\hline & $\operatorname{rr} 12$ & 0.65 & 0.65 & 0.64 & 0.65 & 0.07 \\
\hline & rr22 & 0.61 & 0.61 & 0.61 & 0.61 & 0.07 \\
\hline
\end{tabular}


Tabela C.15: Estatísticas obtidas a partir de 2000 amostras bootstrap para seleção dos pontos de corte e estimação dos riscos relativos, cij e rrij, no caso de tricotomia, em que o ponto de corte $i=1,2$ é estimado pelo método $j=1$,2,3. $O$ indice $i=1$ indica o limite entre os grupos 1 e 2 da FE e $o$ indice $i=2$ indica o limite entre os grupos 2 e 3 da FE. O indice $j$ refere-se ao método MPMINj. Além disso, o procedimento foi realizada para cada um dos 3 cenários avaliados. Todas as estatísticas para os valores-p foram inferiores a 0.01 e, portanto, foram omitidas da tabela.

\begin{tabular}{|c|c|c|c|c|c|c|}
\hline Cenário & Medida & Média & Média aparada & Moda & Mediana & Desvio padrão \\
\hline \multirow{8}{*}{ M1 } & c11 & 26.58 & 26.30 & 25.00 & 25.00 & 1.99 \\
\hline & $c 21$ & 37.98 & 35.98 & 30.00 & 35.00 & 8.27 \\
\hline & $\mathrm{c} 12$ & 26.59 & 26.43 & 25.00 & 25.00 & 1.85 \\
\hline & $\mathrm{c} 22$ & 38.37 & 36.46 & 35.00 & 36.00 & 7.95 \\
\hline & $\operatorname{rr} 11$ & 0.69 & 0.69 & 0.69 & 0.69 & 0.07 \\
\hline & rr21 & 0.53 & 0.52 & 0.49 & 0.52 & 0.05 \\
\hline & $\operatorname{rr} 12$ & 0.69 & 0.68 & 0.69 & 0.68 & 0.07 \\
\hline & $\operatorname{rr} 22$ & 0.52 & 0.52 & 0.51 & 0.52 & 0.05 \\
\hline \multirow{8}{*}{ M2 } & c11 & 26.40 & 26.23 & 25.00 & 25.00 & 1.86 \\
\hline & $c 21$ & 38.21 & 37.18 & 30.00 & 35.00 & 8.76 \\
\hline & $\mathrm{c} 12$ & 26.54 & 26.42 & 25.00 & 25.00 & 1.82 \\
\hline & $\mathrm{c} 22$ & 39.36 & 38.21 & 35.00 & 36.00 & 8.57 \\
\hline & $\operatorname{rr} 11$ & 0.68 & 0.68 & 0.66 & 0.67 & 0.07 \\
\hline & $\operatorname{rr} 21$ & 0.51 & 0.51 & 0.49 & 0.51 & 0.04 \\
\hline & $\operatorname{rr} 12$ & 0.67 & 0.67 & 0.65 ou 0.68 & 0.67 & 0.07 \\
\hline & rr22 & 0.51 & 0.51 & 0.51 & 0.51 & 0.05 \\
\hline \multirow{12}{*}{ Uni } & c11 & 26.87 & 26.53 & 25.00 & 27.00 & 1.91 \\
\hline & $\mathrm{c} 21$ & 38.55 & 36.94 & 30.00 & 36.00 & 7.57 \\
\hline & c12 & 26.55 & 26.36 & 25.00 & 25.00 & 1.74 \\
\hline & $\mathrm{c} 22$ & 37.43 & 35.62 & 30.00 & 36.00 & 7.23 \\
\hline & c13 & 26.54 & 26.36 & 25.00 & 25.00 & 1.74 \\
\hline & $\mathrm{c} 23$ & 37.53 & 35.70 & 30.00 & 36.00 & 7.26 \\
\hline & $\operatorname{rr} 11$ & 0.67 & 0.67 & 0.67 & 0.67 & 0.07 \\
\hline & rr21 & 0.52 & 0.51 & 0.52 & 0.51 & 0.05 \\
\hline & $\operatorname{rr} 12$ & 0.69 & 0.68 & 0.67 & 0.68 & 0.08 \\
\hline & rr22 & 0.51 & 0.51 & 0.52 & 0.51 & 0.05 \\
\hline & $\operatorname{rr} 13$ & 0.69 & 0.68 & 0.66 & 0.68 & 0.08 \\
\hline & rr23 & 0.51 & 0.51 & 0.52 & 0.51 & 0.05 \\
\hline
\end{tabular}


Tabela C.16: Estatísticas obtidas a partir de 1000 repetições do método de validação cruzada para obtenção dos pontos de corte e estimação dos riscos relativos, cij e rrij, no caso de tricotomia, em que o ponto de corte $i=1,2$ é estimado pelo método $j=1$,2. $O$ indice $i=1$ indica o limite entre os grupos 1 e 2 da FE e o indice $i=2$ indica o limite entre os grupos 2 e 3 da FE. $O$ indice $j$ refere-se ao método MDVj. Além disso, o procedimento foi realizada para cada um dos 3 cenários avaliados.

\begin{tabular}{ccccccc} 
Cenário & Medida & Média & Média aparada & Moda & Mediana & Desvio padrão \\
\hline \multirow{3}{*}{ M1 } & rr11 & 0.66 & 0.65 & 0.65 & 0.65 & 0.04 \\
& $\operatorname{rr} 21$ & 0.61 & 0.61 & 0.60 & 0.61 & 0.03 \\
& $\operatorname{rr} 12$ & 0.64 & 0.64 & 0.64 & 0.64 & 0.04 \\
& $\operatorname{rr} 22$ & 0.61 & 0.61 & 0.61 & 0.61 & 0.04 \\
\multirow{3}{*}{ M2 } & $\operatorname{rr} 11$ & 0.65 & 0.65 & 0.65 & 0.65 & 0.04 \\
& $\operatorname{rr} 21$ & 0.59 & 0.59 & 0.59 & 0.59 & 0.03 \\
& $\operatorname{rr} 12$ & 0.63 & 0.63 & 0.63 & 0.63 & 0.04 \\
& $\operatorname{rr} 22$ & 0.59 & 0.59 & 0.59 & 0.59 & 0.04 \\
\multirow{3}{*}{ Uni } & $\operatorname{rr} 11$ & 0.65 & 0.65 & 0.65 & 0.65 & 0.04 \\
& $\operatorname{rr} 21$ & 0.60 & 0.60 & 0.61 & 0.61 & 0.03 \\
& $\operatorname{rr} 12$ & 0.63 & 0.63 & 0.63 & 0.63 & 0.04 \\
& $\operatorname{rr} 22$ & 0.61 & 0.61 & 0.61 & 0.61 & 0.04
\end{tabular}

Tabela C.17: Estatísticas obtidas a partir de 1000 repetições do método de validação cruzada para estimação dos riscos relativos e valores-p, rrij e pj, no caso de tricotomia, em que o ponto de corte $i=1,2$ é estimado pelo método $j=1,2,3 . O$ indice $i=1$ indica o limite entre os grupos 1 e 2 da FE e o indice $i=2$ indica o limite entre os grupos 2 e 3 da FE. $O$ índice $j$ refere-se ao método MPMINj. Além disso, o procedimento foi realizada para cada um dos 3 cenários avaliados. Todas as estatisticas para os valores-p foram inferiores a 0.01 e, portanto, foram omitidas da tabela, exceto para o cenário M1.

Cenário Medida Média Média aparada Moda Mediana Desvio padrão

\begin{tabular}{|c|c|c|c|c|c|c|}
\hline \multirow{4}{*}{ M1 } & rr11 & 0.67 & 0.67 & 0.67 & 0.67 & 0.03 \\
\hline & $\operatorname{rr} 21$ & 0.57 & 0.57 & 0.57 & 0.57 & 0.02 \\
\hline & $\operatorname{rr} 12$ & 0.64 & 0.64 & 0.65 & 0.63 & 0.03 \\
\hline & $\operatorname{rr} 22$ & 0.59 & 0.59 & 0.59 & 0.59 & 0.03 \\
\hline \multirow{4}{*}{ M2 } & rr11 & 0.66 & 0.66 & 0.66 & 0.66 & 0.03 \\
\hline & $\operatorname{rr} 21$ & 0.56 & 0.56 & 0.56 & 0.56 & 0.02 \\
\hline & $\operatorname{rr} 12$ & 0.62 & 0.62 & 0.62 & 0.62 & 0.03 \\
\hline & $\operatorname{rr} 22$ & 0.58 & 0.58 & 0.57 & 0.57 & 0.03 \\
\hline \multirow{6}{*}{ Uni } & $\operatorname{rr} 11$ & 0.67 & 0.67 & 0.66 & 0.67 & 0.03 \\
\hline & rr21 & 0.56 & 0.56 & 0.55 & 0.56 & 0.02 \\
\hline & $\operatorname{rr} 12$ & 0.62 & 0.62 & 0.61 & 0.62 & 0.03 \\
\hline & $\operatorname{rr} 22$ & 0.57 & 0.57 & 0.57 & 0.57 & 0.03 \\
\hline & $\operatorname{rr} 13$ & 0.62 & 0.62 & 0.62 & 0.62 & 0.03 \\
\hline & $\operatorname{rr} 23$ & 0.57 & 0.57 & 0.57 & 0.57 & 0.03 \\
\hline
\end{tabular}


Tabela C.18: Fatores de contração heurístico, bootstrap e validação cruzada para o cenário univariado no caso de dicotomia.

\begin{tabular}{ccccc} 
Fator de contração & Método & $c$ & $\hat{d}$ & $\widehat{r r}$ \\
\hline \multirow{3}{*}{ Heurístico } & MDV1 & 32 & 0.9778 & 0.6423 \\
& MDV2 & 26 & 0.9809 & 0.5980 \\
& MPMIN & 28 & 0.9835 & 0.5846 \\
Heurístico* & MDV1 & 31 & 0.9805 & 0.6220 \\
& MDV2 & 36 & 0.9759 & 0.6492 \\
& MPMIN1 & 30 & 0.9828 & 0.6024 \\
Bootstrap1 & MIN2/MPMIN3 & 28 & 0.9835 & 0.5846 \\
& MPMIN2 & - & - & 0.6020 \\
Bootstrap2 & MPMIN3 & - & - & 0.6001 \\
& MPMIN1 & - & 0.9317 & 0.6013 \\
& MPMIN2 & - & 0.9377 & 0.5993 \\
Validação Cruzada & MPMIN3 & - & 0.9375 & 0.5994 \\
& MDV1 & 32 & 0.9700 & 0.6445 \\
& MDV2 & 26 & 0.9640 & 0.6032 \\
& MPMIN & 28 & 0.9724 & 0.5882 \\
Validação Cruzada* & MDV1 & 31 & 0.9657 & 0.6265 \\
& MDV2 & 36 & 0.9646 & 0.6525 \\
& MPMIN1 & 30 & 0.9646 & 0.6081 \\
& MPMIN2/MPMIN3 & 28 & 0.9724 & 0.5882 \\
\hline
\end{tabular}

${ }^{\mathrm{i}}$ Heurístico* indica o fator de contração heurístico aplicado aos pontos de corte validados pelo método boot.

ii Validação cruzada* indica o fator de contração validação cruzada aplicado aos pontos de corte validados pelo método boot. 


\section{Apêndice D}

\section{Histogramas da distribuição dos pontos de corte e riscos relativos estimados pelo método boot}

Figura D.1: Histogramas da distribuição dos pontos de corte e riscos relativos estimados pelo método de validação boot no caso de dicotomia para o método MDV - caso 1

Figura D.2: Histogramas da distribuição dos pontos de corte e riscos relativos estimados pelo método de validação boot no caso de dicotomia para o método MPMIN - caso 1

Figura D.3: Histogramas da distribuição dos pontos de corte e riscos relativos estimados pelo método de validação boot no caso de tricotomia para o método MDV1 - caso 1

Figura D.4: Histogramas da distribuição dos pontos de corte e riscos relativos estimados pelo método de validação boot no caso de tricotomia para o método MDV2 - caso 1

Figura D.5: Histogramas da distribuição dos pontos de corte e riscos relativos estimados pelo método de validação boot no caso de tricotomia para o método MPMIN1 - caso 1

Figura D.6: Histogramas da distribuição dos pontos de corte e riscos relativos estimados pelo método de validação boot no caso de tricotomia para o método MPMIN2 - caso 1

Figura D.7: Histogramas da distribuição dos pontos de corte e riscos relativos estimados pelo método de validação boot no caso de tricotomia para o método MPMIN3 - caso 1

Figura D.8: Histogramas da distribuição dos pontos de corte e riscos relativos estimados pelo método de validação boot no caso de dicotomia para o método MDV - caso 2

Figura D.9: Histogramas da distribuição dos pontos de corte e riscos relativos estimados pelo método de validação boot no caso de dicotomia para o método MPMIN - caso 2

Figura D.10: Histogramas da distribuição dos pontos de corte e riscos relativos estimados pelo método de validação boot no caso de tricotomia para o método MDV1 - caso 2

Figura D.11: Histogramas da distribuição dos pontos de corte e riscos relativos estimados pelo método de validação boot no caso de tricotomia para o método MDV2 - caso 2

Figura D.12: Histogramas da distribuição dos pontos de corte e riscos relativos estimados pelo método de validação boot no caso de tricotomia para o método MPMIN1 - caso 2

Figura D.13: Histogramas da distribuição dos pontos de corte e riscos relativos estimados pelo método de validação boot no caso de tricotomia para o método MPMIN2 - caso 2

Figura D.14: Histogramas da distribuição dos pontos de corte e riscos relativos estimados pelo método de validação boot no caso de tricotomia para o método MPMIN3 - caso 2 

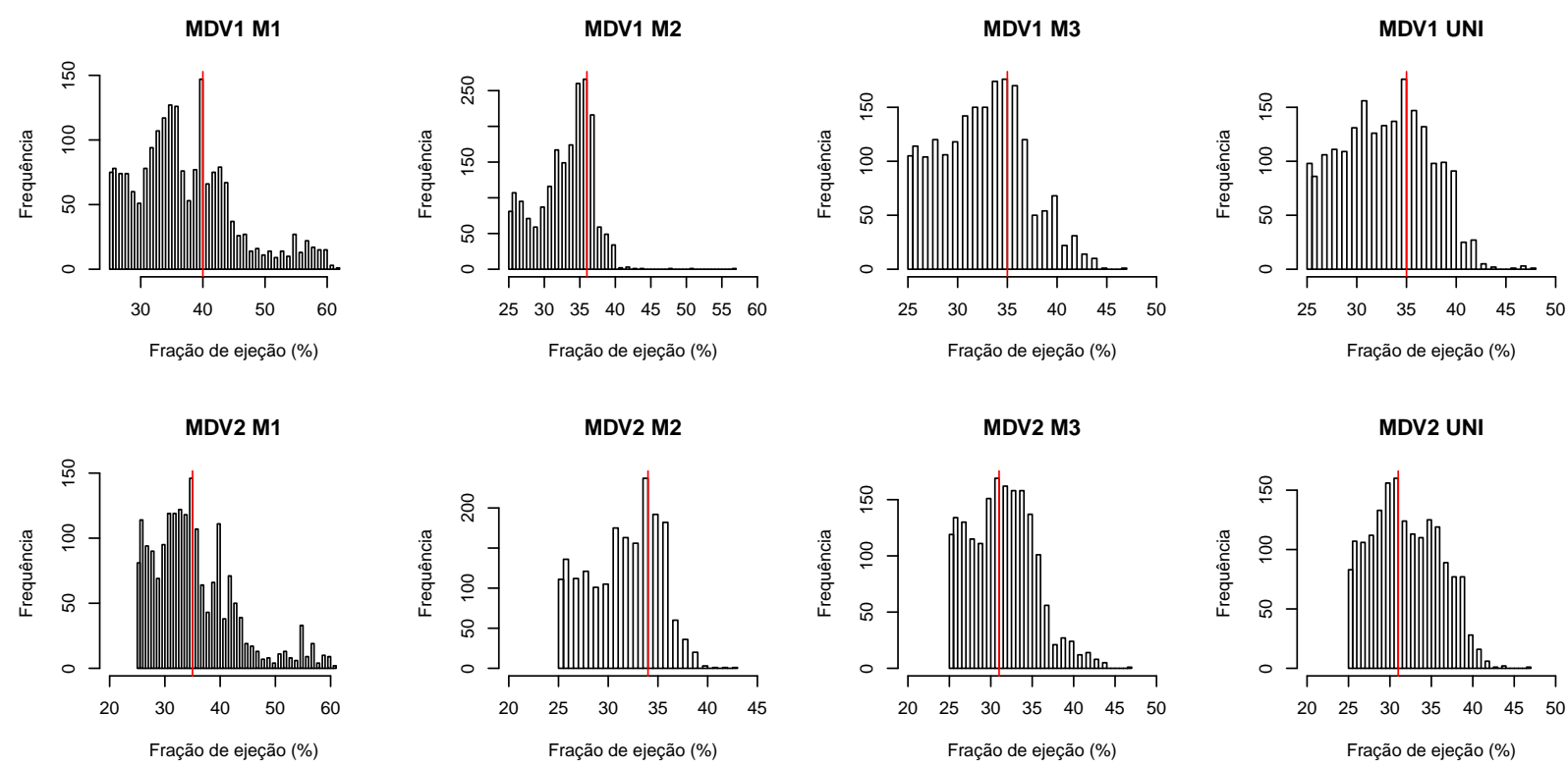

(a) Figura 1: Histogramas referentes aos pontos de corte estimados
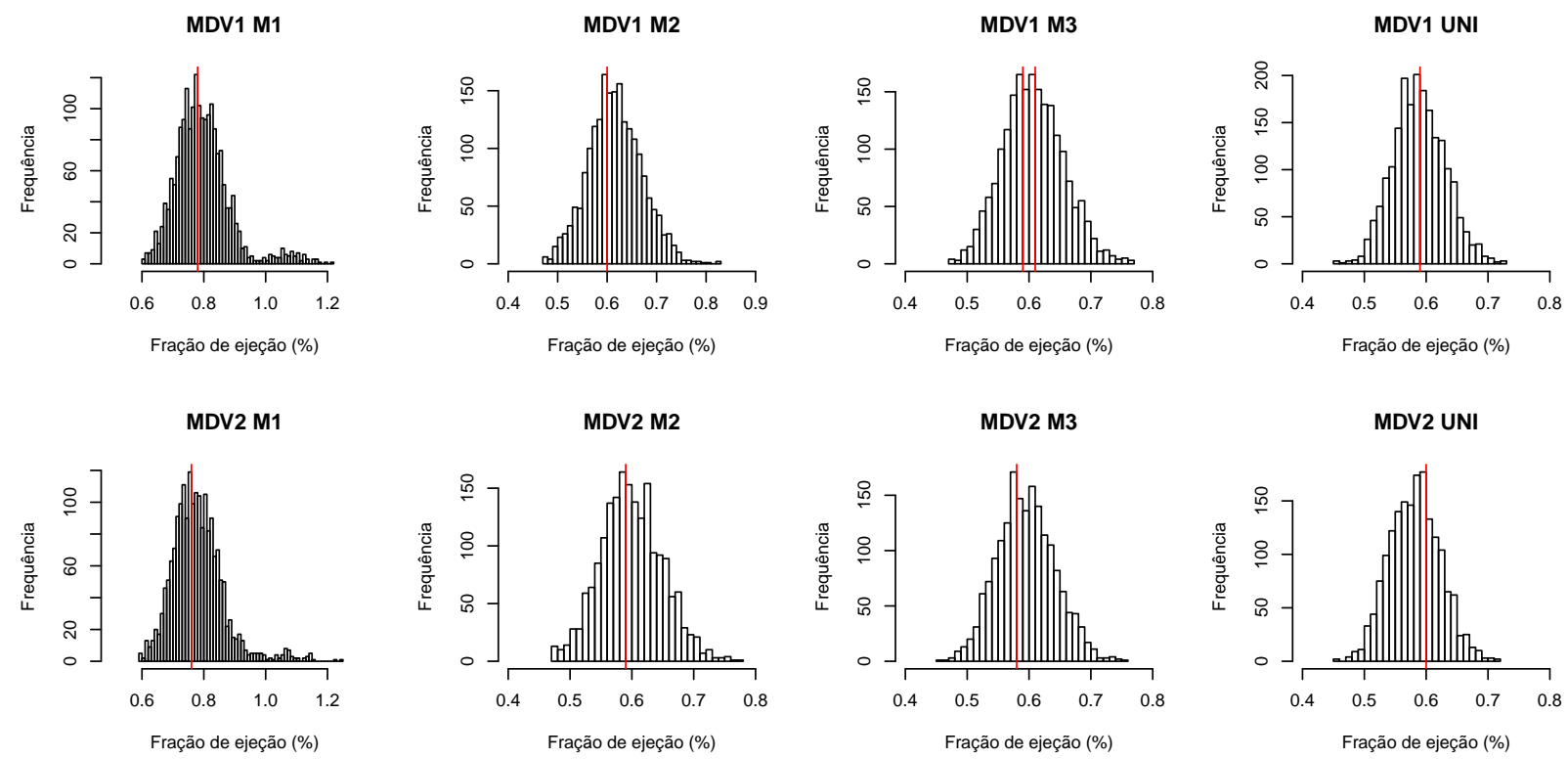

(b) Figura 2: Histogramas referentes aos riscos relativos estimados

Figura D.1: As figuras 1 e 2 apresentam os histogramas da distribuição dos pontos de corte e riscos relativos estimados pelo método de validação bootstrap para os métodos de seleção de pontos de corte MDV1 e MDV2, nos cenários M1, M2, M3 e Uni, respectivamente. Linha vermelha indica a moda. 

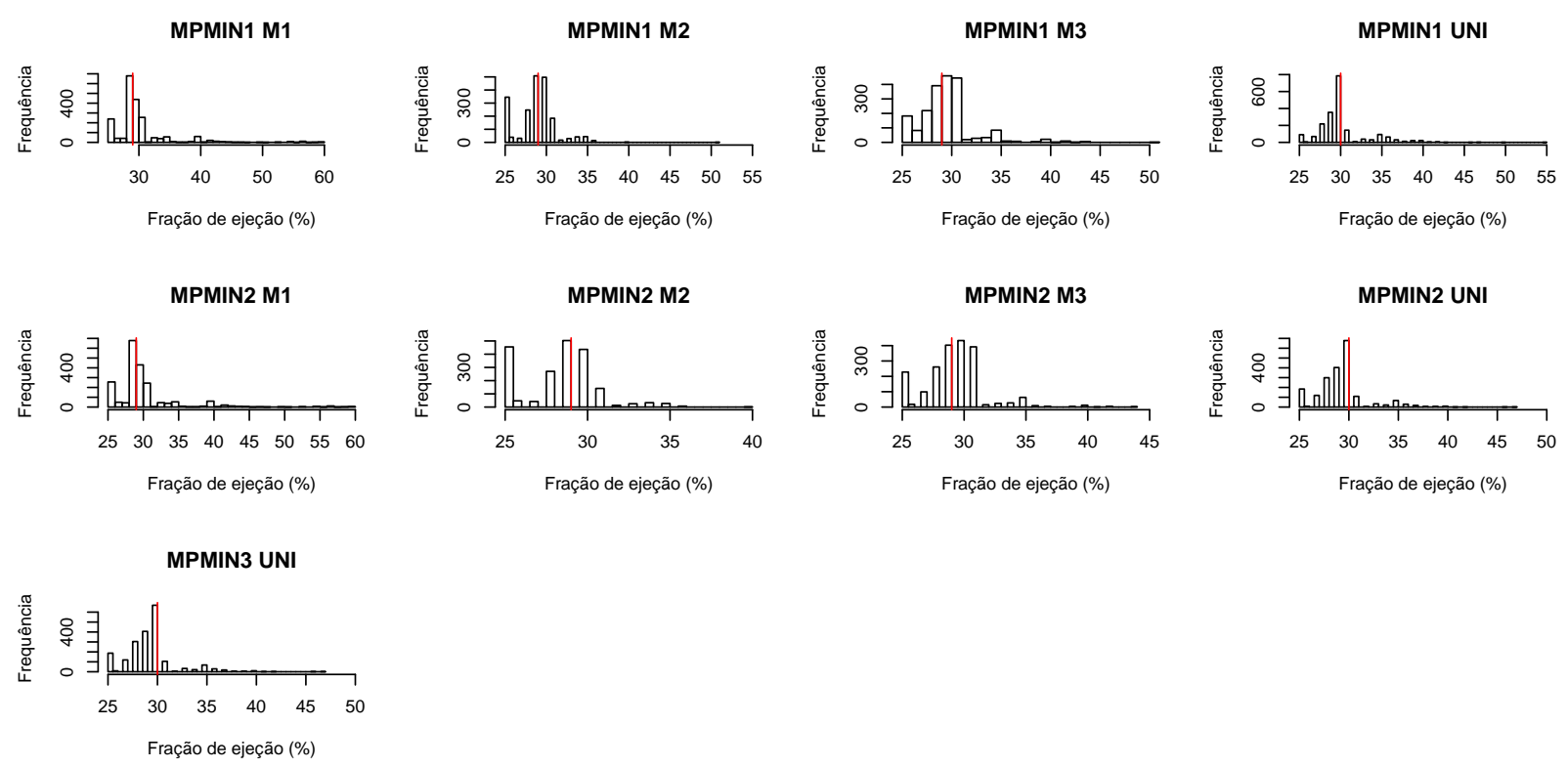

(a) Figura 1: Histogramas referentes aos pontos de corte estimados
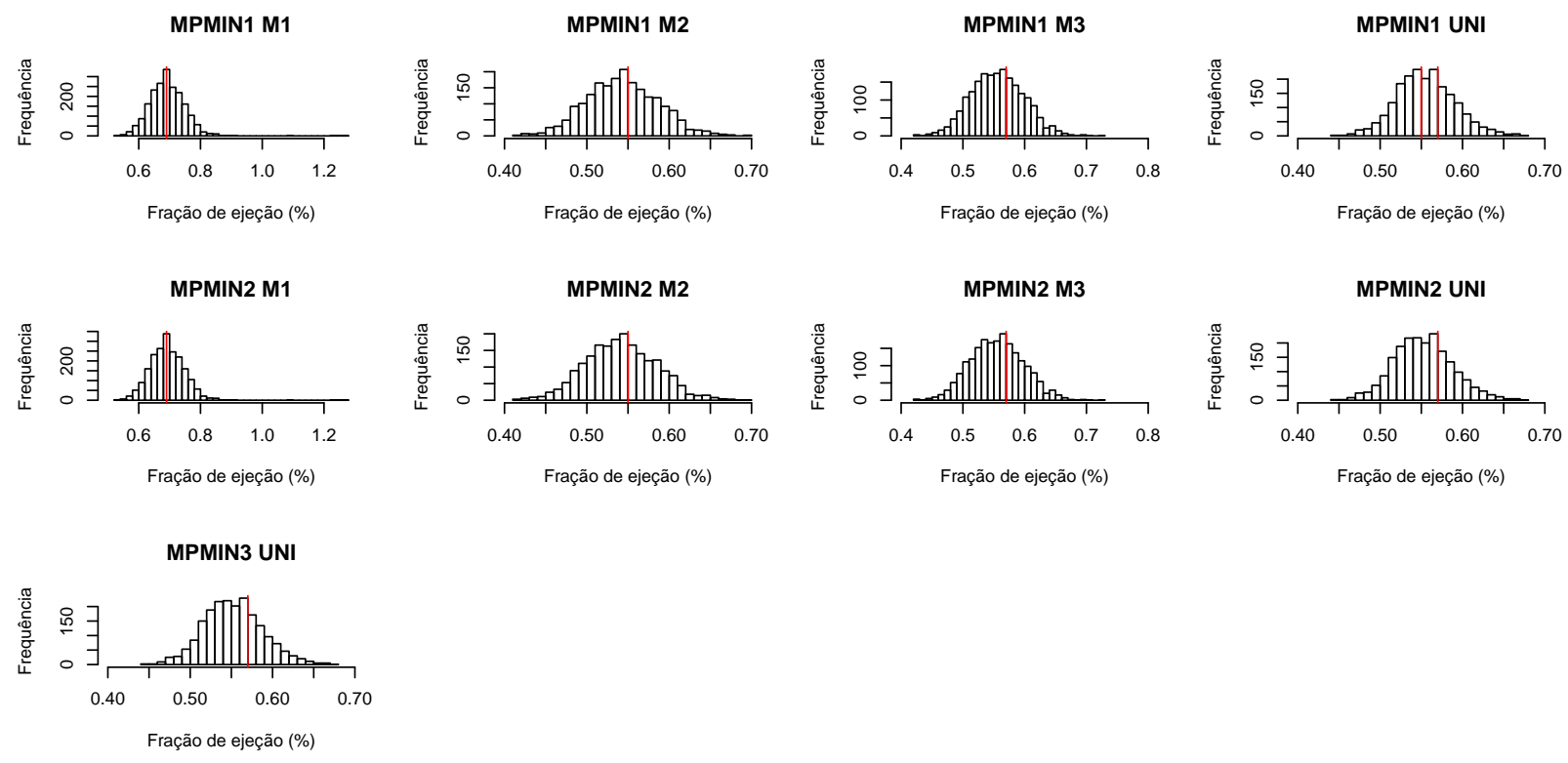

(b) Figura 2: Histogramas referentes aos riscos relativos estimados

Figura D.2: As figuras 1 e 2 apresentam os histogramas da distribuição dos pontos de corte e riscos relativos estimados pelo método de validação bootstrap para os métodos de seleção de pontos de corte MPMIN1, MPMIN2 e MPMIN3 nos cenários M1, M2, M3 e Uni, respectivamente. Linha vermelha indica a moda. 

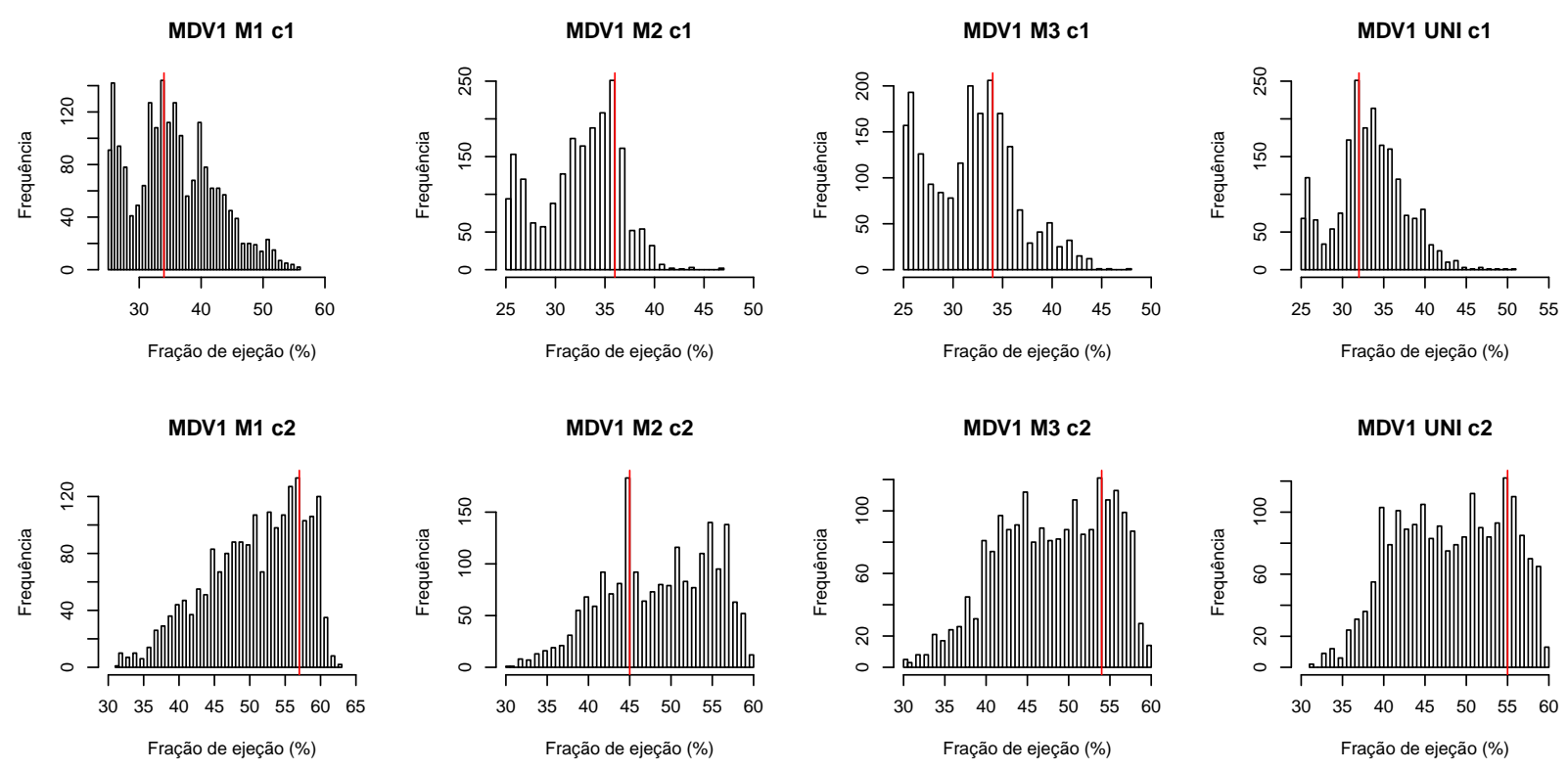

(a) Figura 1: Histogramas referentes aos pontos de corte c1 e c2 estimados
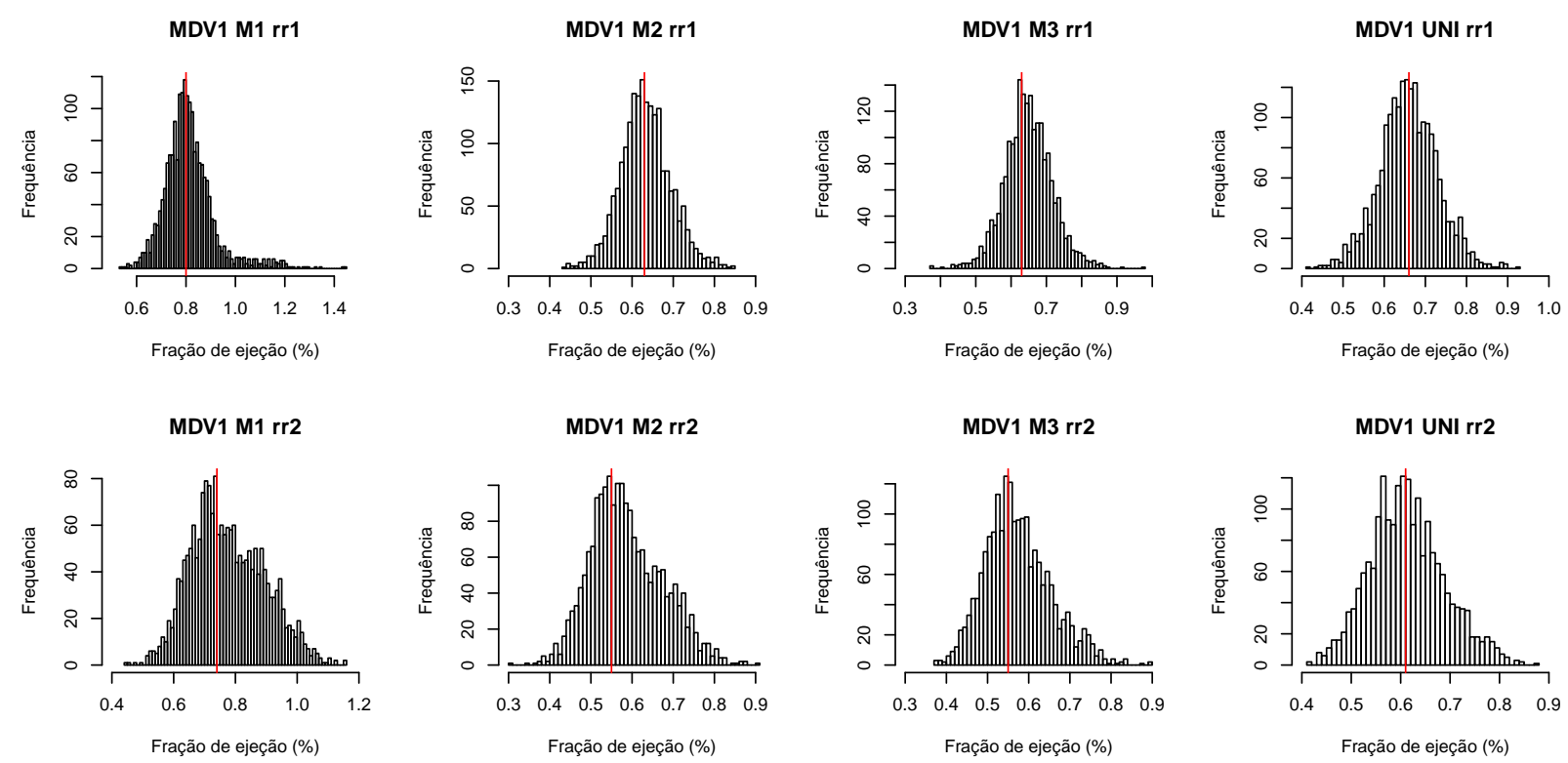

(b) Figura 2: Histogramas referentes aos riscos relativos rr1 e rr2 estimados

Figura D.3: As figuras 1 e 2 apresentam os histogramas da distribuição dos pontos de corte e riscos relativos estimados pelo método de validação bootstrap para o método de seleção de pontos de corte MDV1 nos cenários M1, M2, M3 e Uni, respectivamente. Linha vermelha indica a moda. 

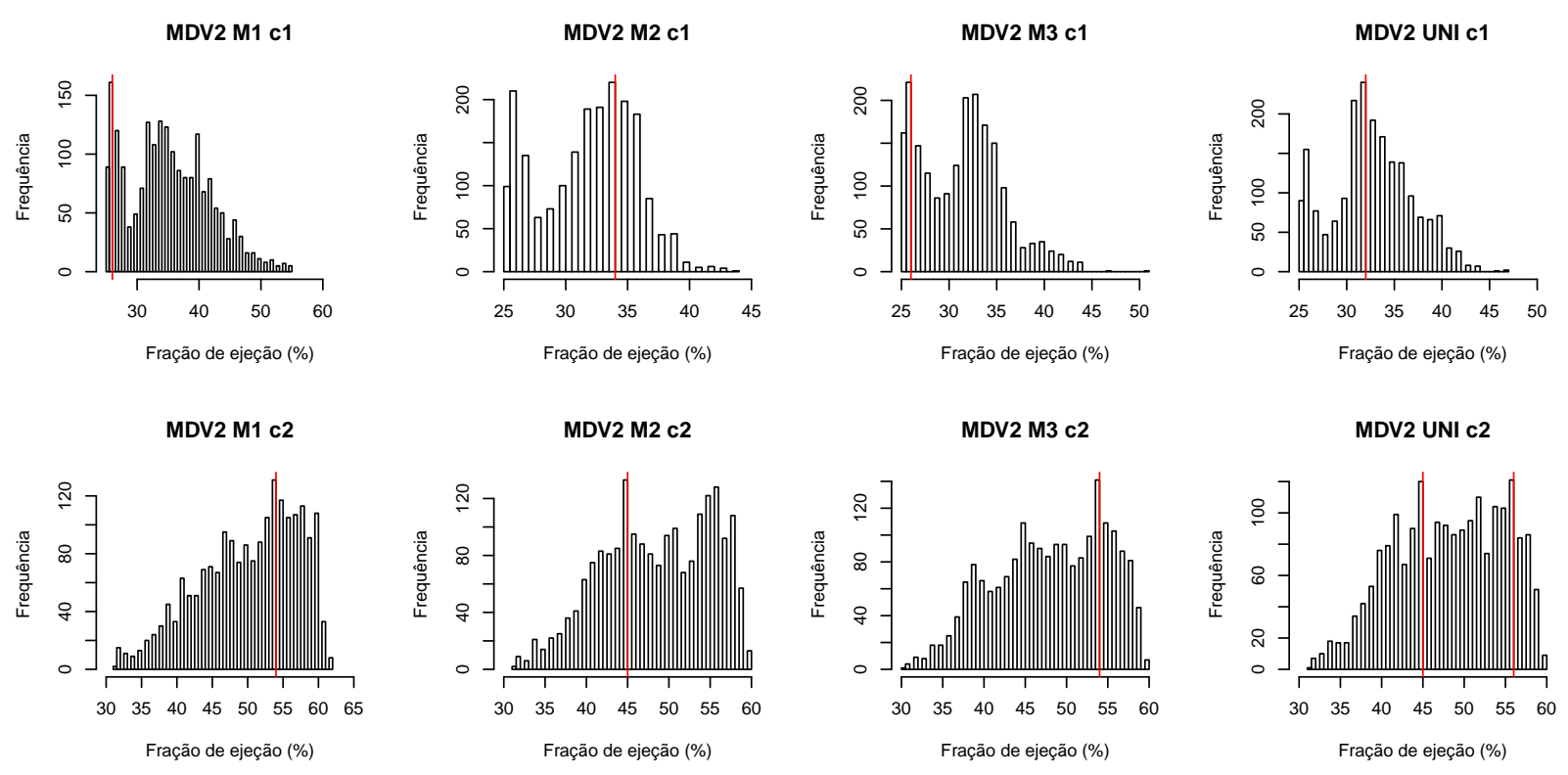

(a) Figura 1: Histogramas referentes aos pontos de corte c1 e c2 estimados
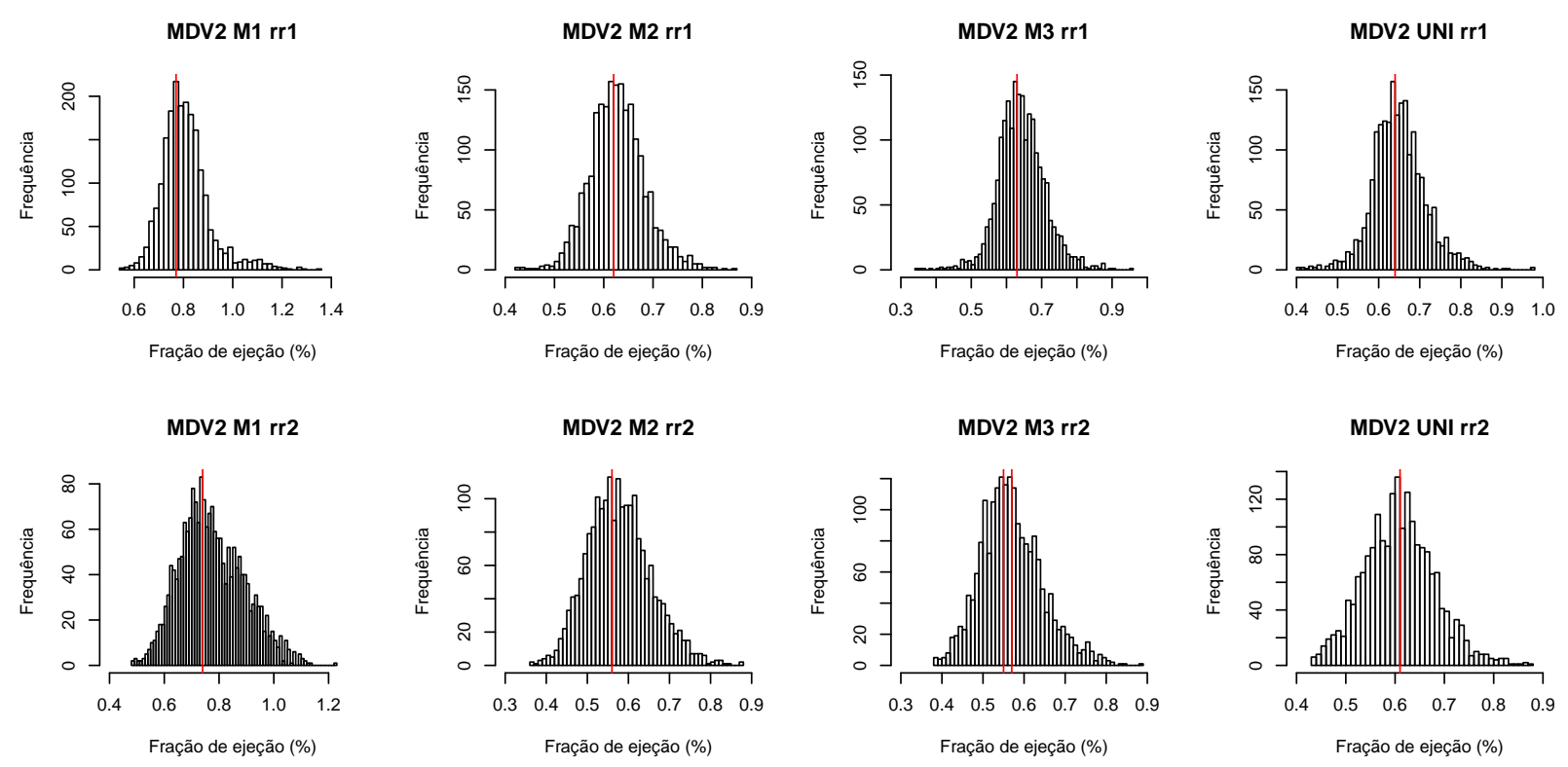

(b) Figura 2: Histogramas referentes aos riscos relativos rr1 e rr2 estimados

Figura D.4: As figuras 1 e 2 apresentam os histogramas da distribuição dos pontos de corte $e$ riscos relativos estimados pelo método de validação bootstrap para o método de seleção de pontos de corte MDV2 nos cenários M1, M2, M3 e Uni, respectivamente. Linha vermelha indica a moda. 

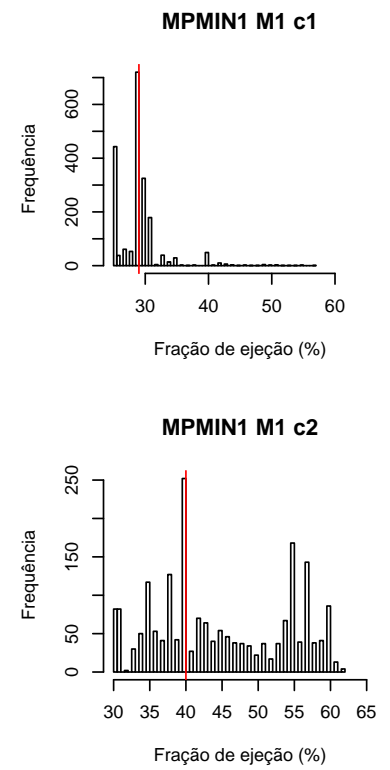

MPMIN1 M2 c1

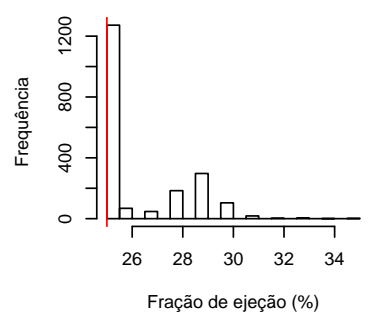

MPMIN1 M2 c2

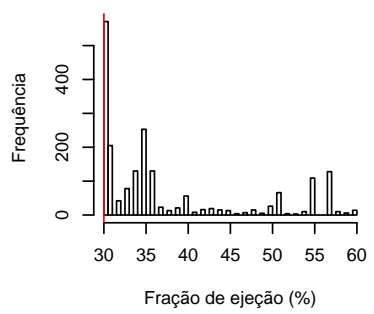

MPMIN1 M3 c1

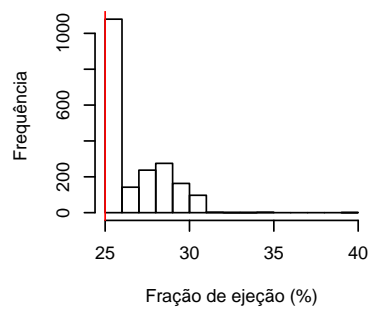

MPMIN1 M3 c2

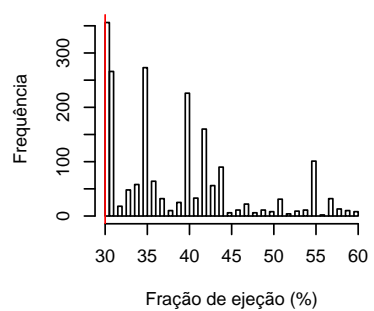

MPMIN1 UNI c1

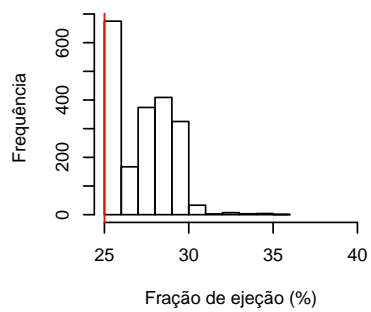

MPMIN1 UNI c2

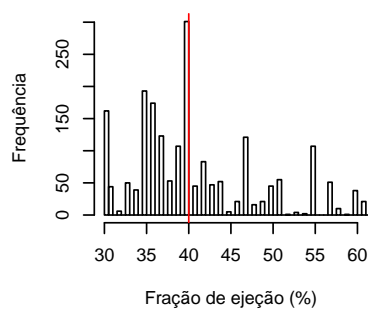

(a) Figura 1: Histogramas referentes aos pontos de corte c1 e c2 estimados

MPMIN1 M1 rr1

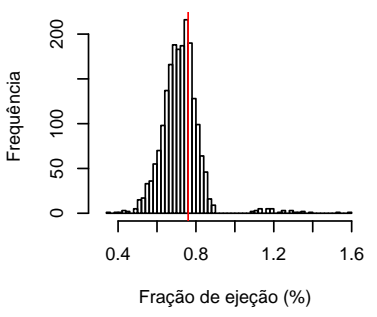

MPMIN1 M1 rr2

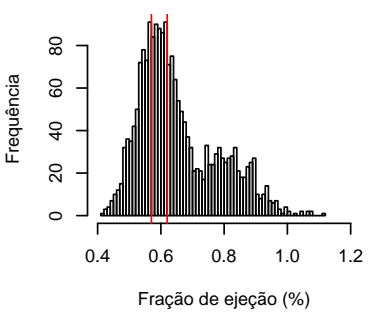

MPMIN1 M2 rr1

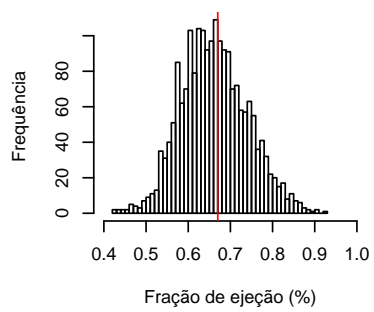

MPMIN1 M2 rr2

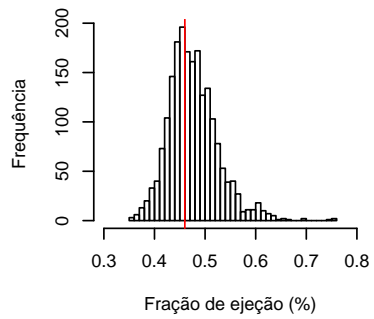

MPMIN1 M3 rr1

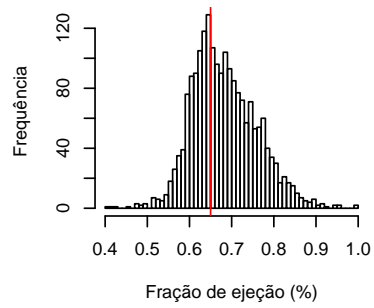

MPMIN1 M3 rr2

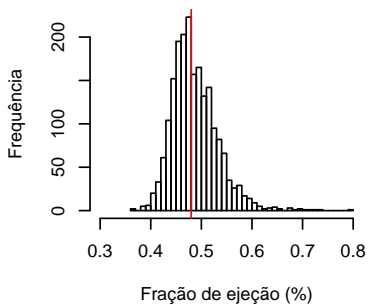

MPMIN1 UNI rr1

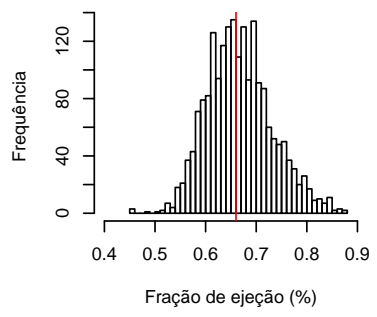

MPMIN1 UNI rr2

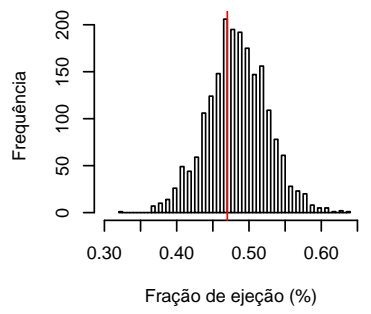

(b) Figura 2: Histogramas referentes aos riscos relativos rr1 e rr2 estimados

Figura D.5: As figuras 1 e 2 apresentam os histogramas da distribuição dos pontos de corte $e$ riscos relativos estimados pelo método de validação bootstrap para o método de seleção de pontos de corte MPMIN1 nos cenários M1, M2, M3 e Uni, respectivamente. Linha vermelha indica a moda. 


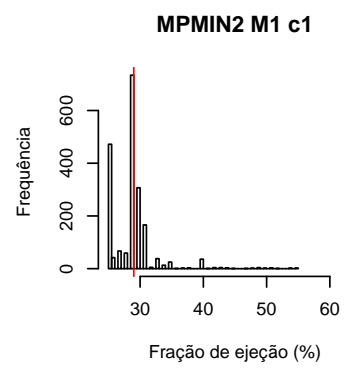

MPMIN2 M1 c2

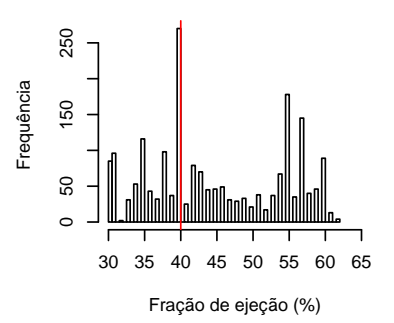

MPMIN2 M2 c1

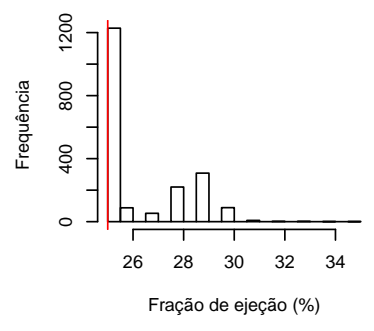

MPMIN2 M2 c2

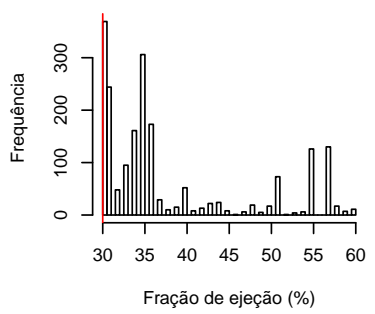

MPMIN2 M3 c1

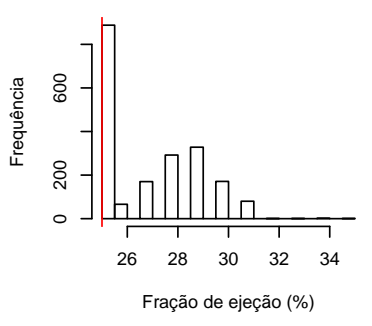

MPMIN2 M3 c2

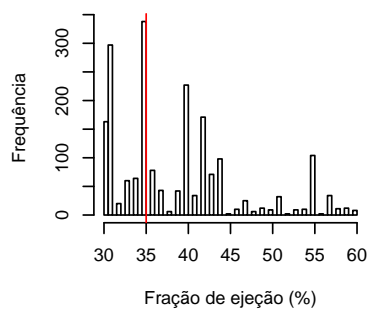

MPMIN2 UNI c1

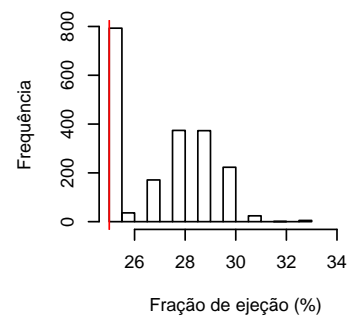

MPMIN2 UNI c2

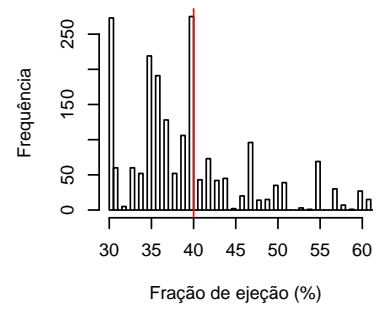

(a) Figura 1: Histogramas referentes aos pontos de corte c1 e c2 estimados

MPMIN2 M1 rr1

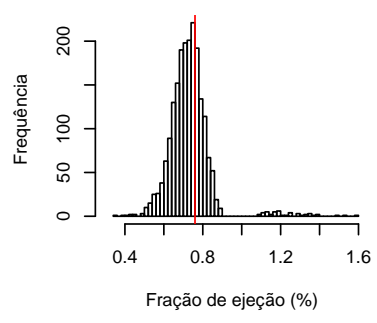

MPMIN2 M1 rr2

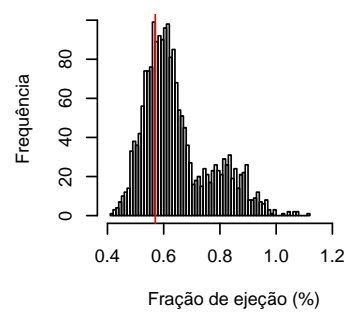

MPMIN2 M2 rr1

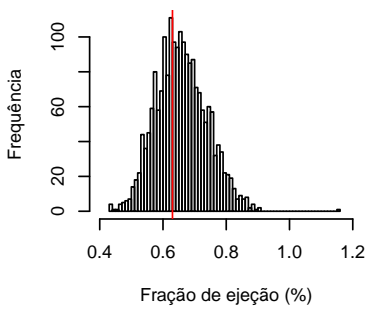

MPMIN2 M2 rr2

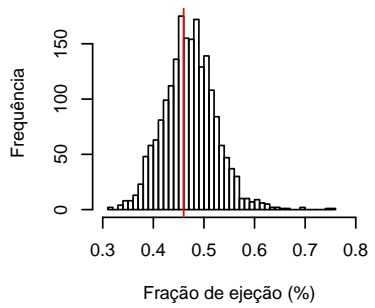

MPMIN2 M3 rr1

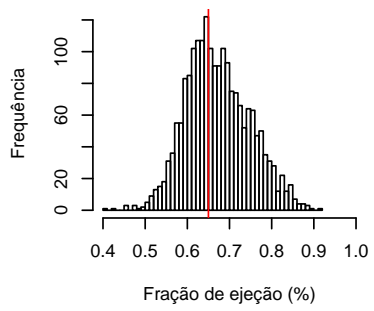

MPMIN2 M3 rr2

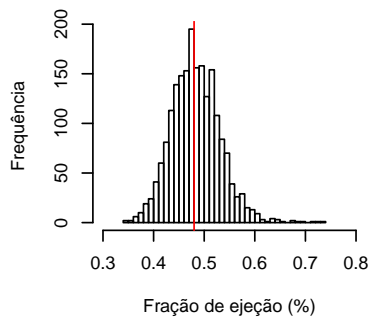

MPMIN2 UNI rr1

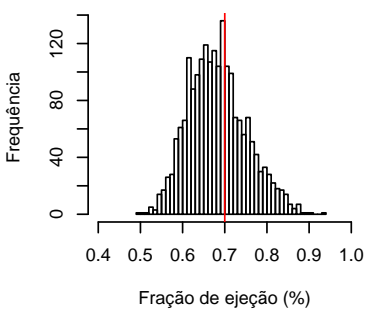

MPMIN2 UNI rr2

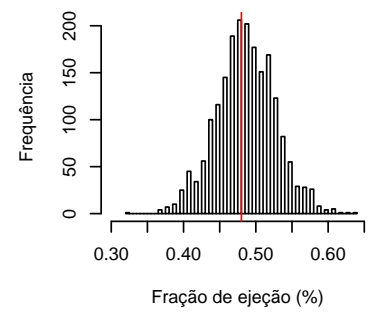

(b) Figura 2: Histogramas referentes aos riscos relativos rr1 e rr2 estimados

Figura D.6: As figuras 1 e 2 apresentam os histogramas da distribuição dos pontos de corte e riscos relativos estimados pelo método de validação bootstrap para o método de seleção de pontos de corte MPMIN2 nos cenários M1, M2, M3 e Uni, respectivamente. Linha vermelha indica a moda. 
MPMIN3 UNI c1

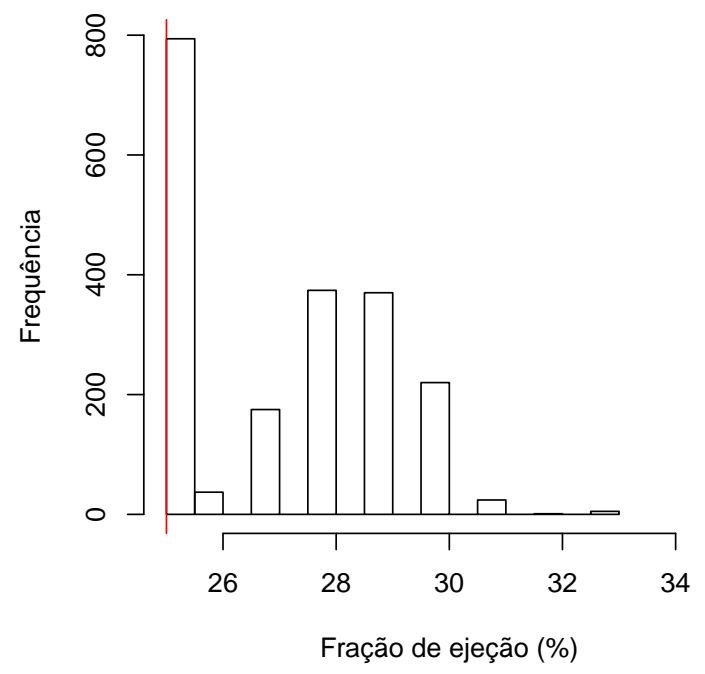

MPMIN3 UNI c2

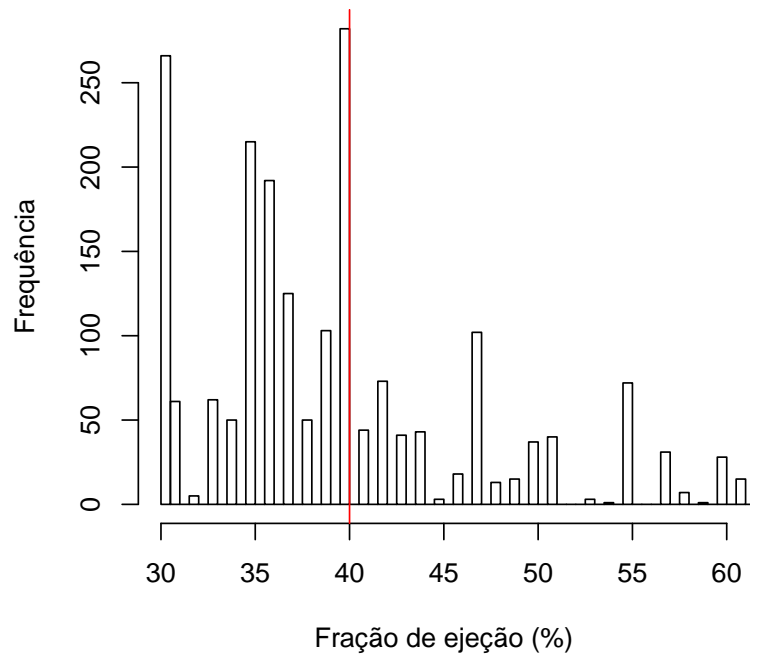

(a) Figura 1: Histogramas referentes aos pontos de corte c1 e c2 estimados

MPMIN3 UNI rr1

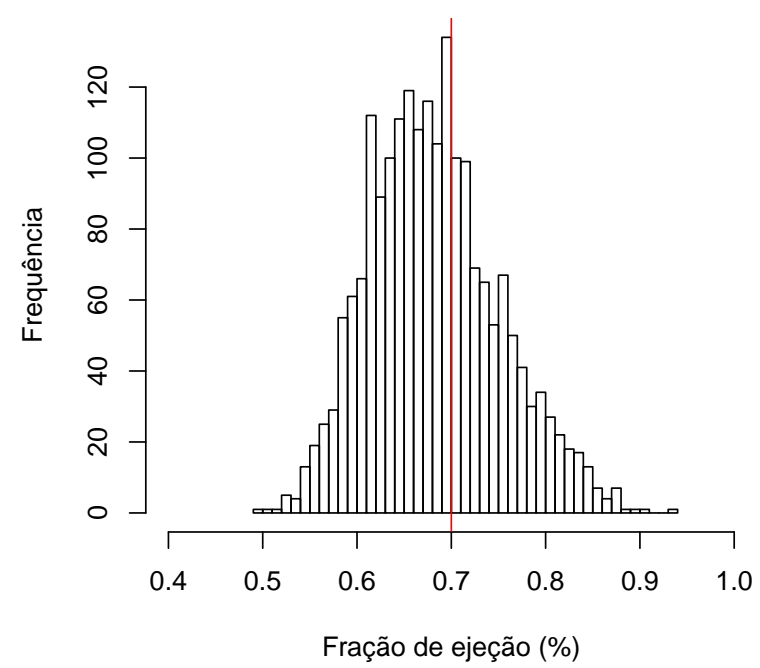

MPMIN3 UNI rr2

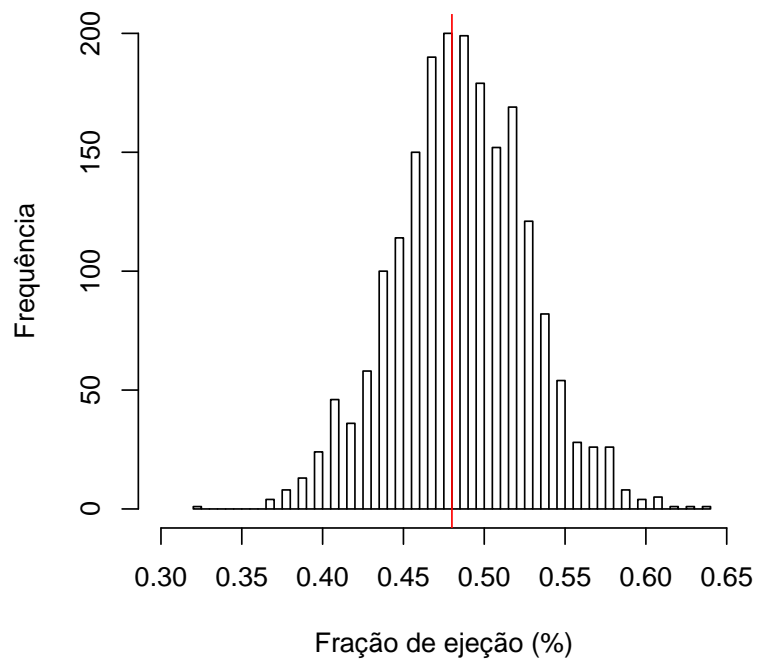

(b) Figura 2: Histogramas referentes aos riscos relativos rr1 e rr2 estimados

Figura D.7: As figuras 1 e 2 apresentam os histogramas da distribuição dos pontos de corte $e$ riscos relativos estimados pelo método de validação bootstrap para o método de seleção de pontos de corte MPMIN3 no cenário Uni, respectivamente. Linha vermelha indica a moda. 

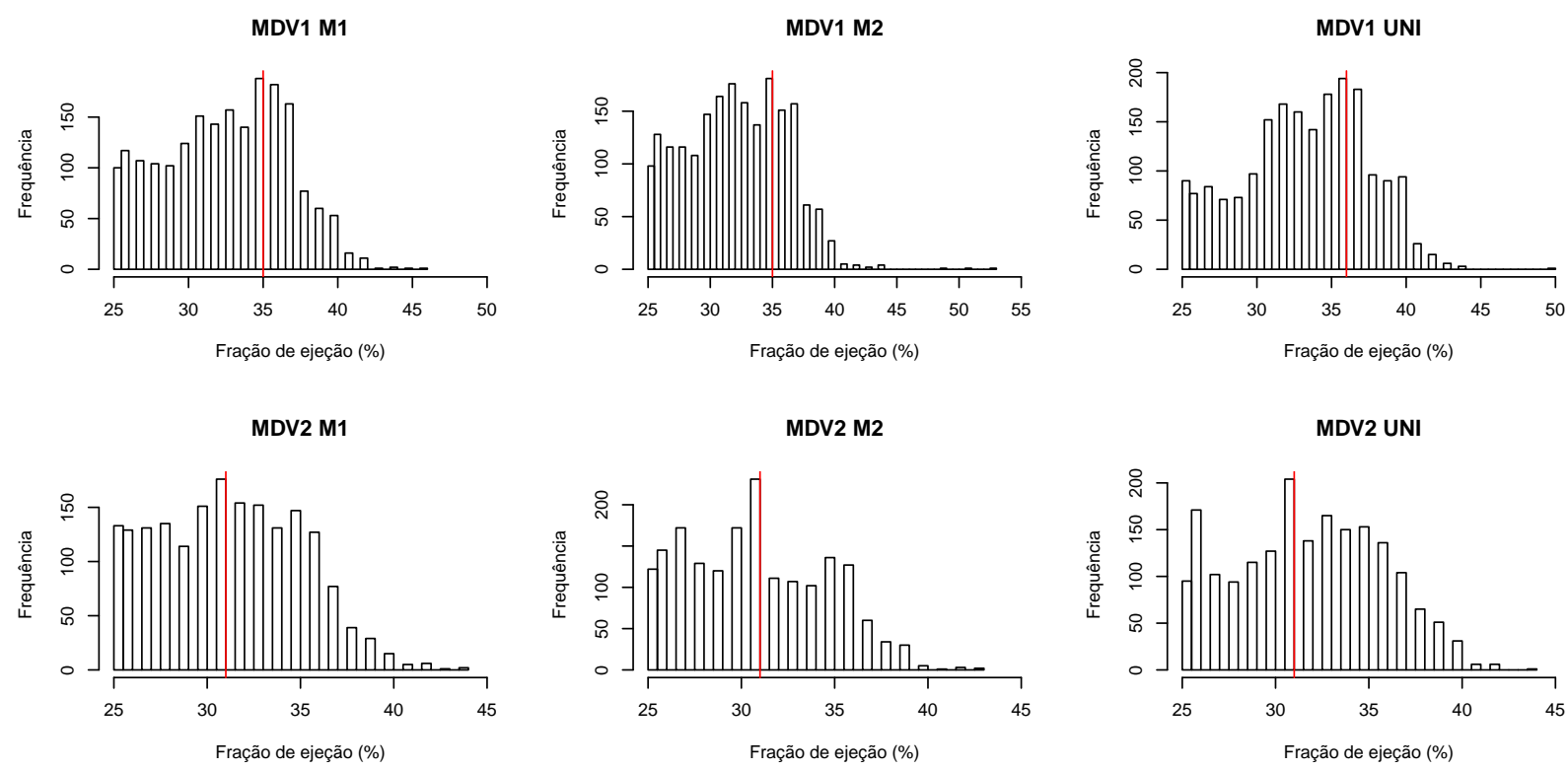

(a) Figura 1: Histogramas referentes aos pontos de corte estimados
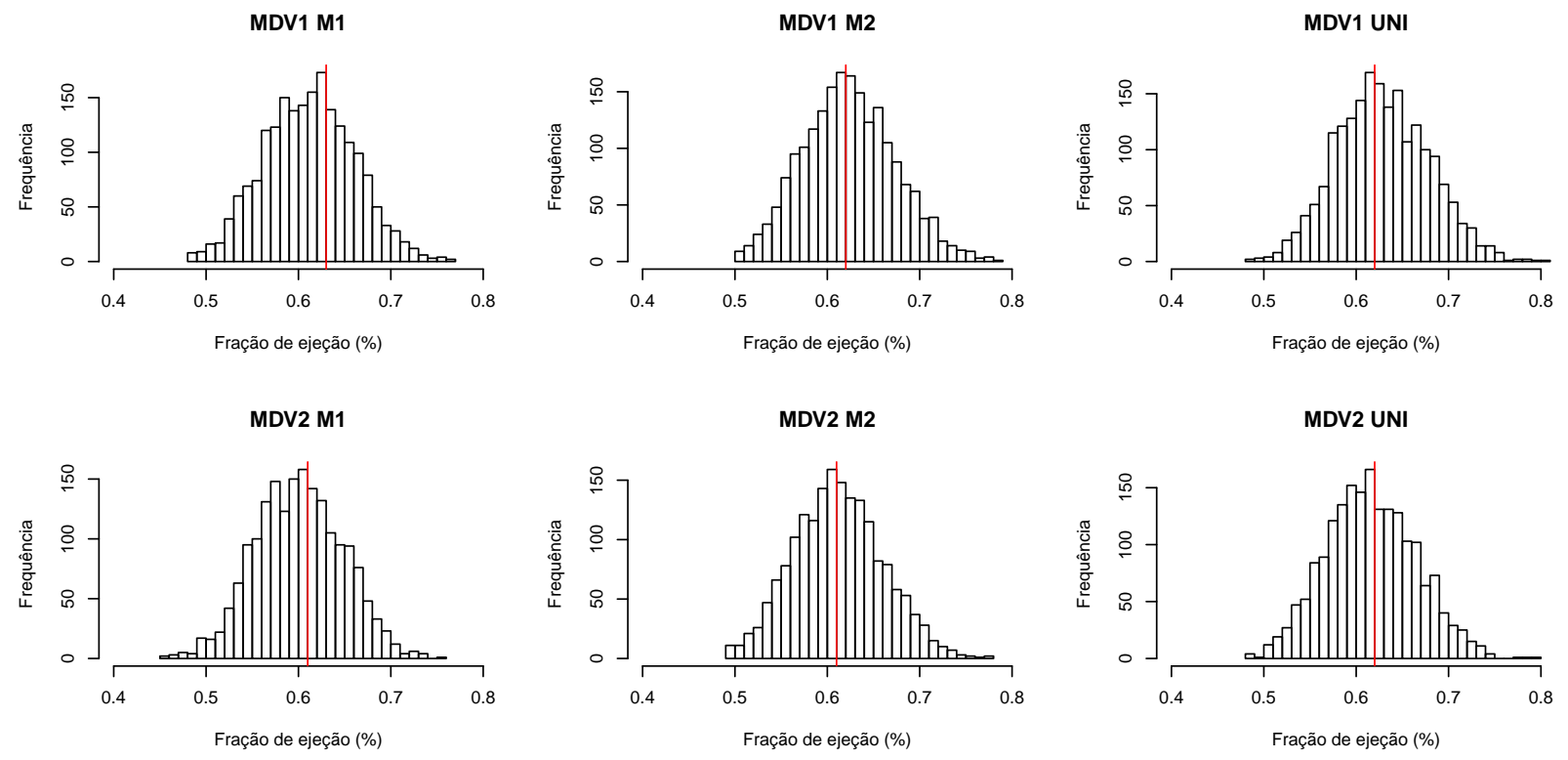

(b) Figura 2: Histogramas referentes aos riscos relativos estimados

Figura D.8: As figuras 1 e 2 apresentam os histogramas da distribuição dos pontos de corte $e$ riscos relativos estimados pelo método de validação bootstrap para os métodos de seleção de pontos de corte MDV1 e MDV2, nos cenários M1, M2 e Uni, respectivamente. Linha vermelha indica a moda. (caso2) 
MPMIN1 M1

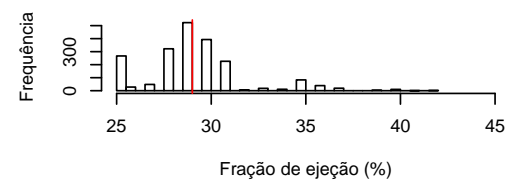

MPMIN2 M1

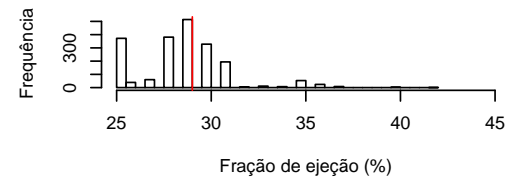

MPMIN3 UNI

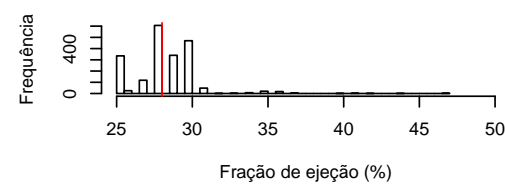

MPMIN1 M2

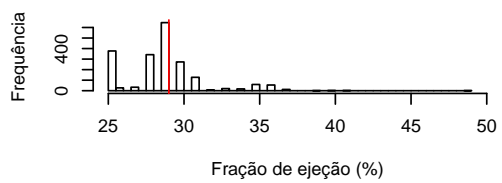

MPMIN2 M2

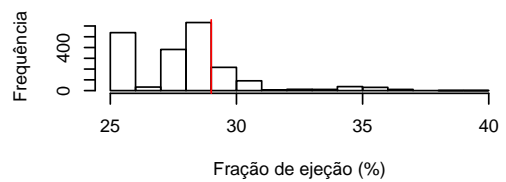

MPMIN1 UNI

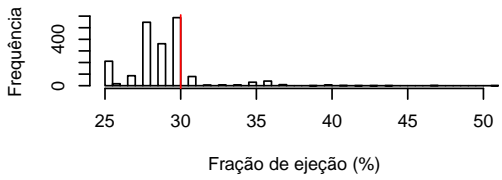

MPMIN2 UNI

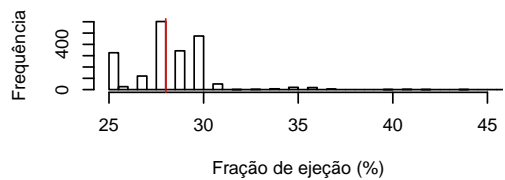

(a) Figura 1: Histogramas referentes aos pontos de corte estimados
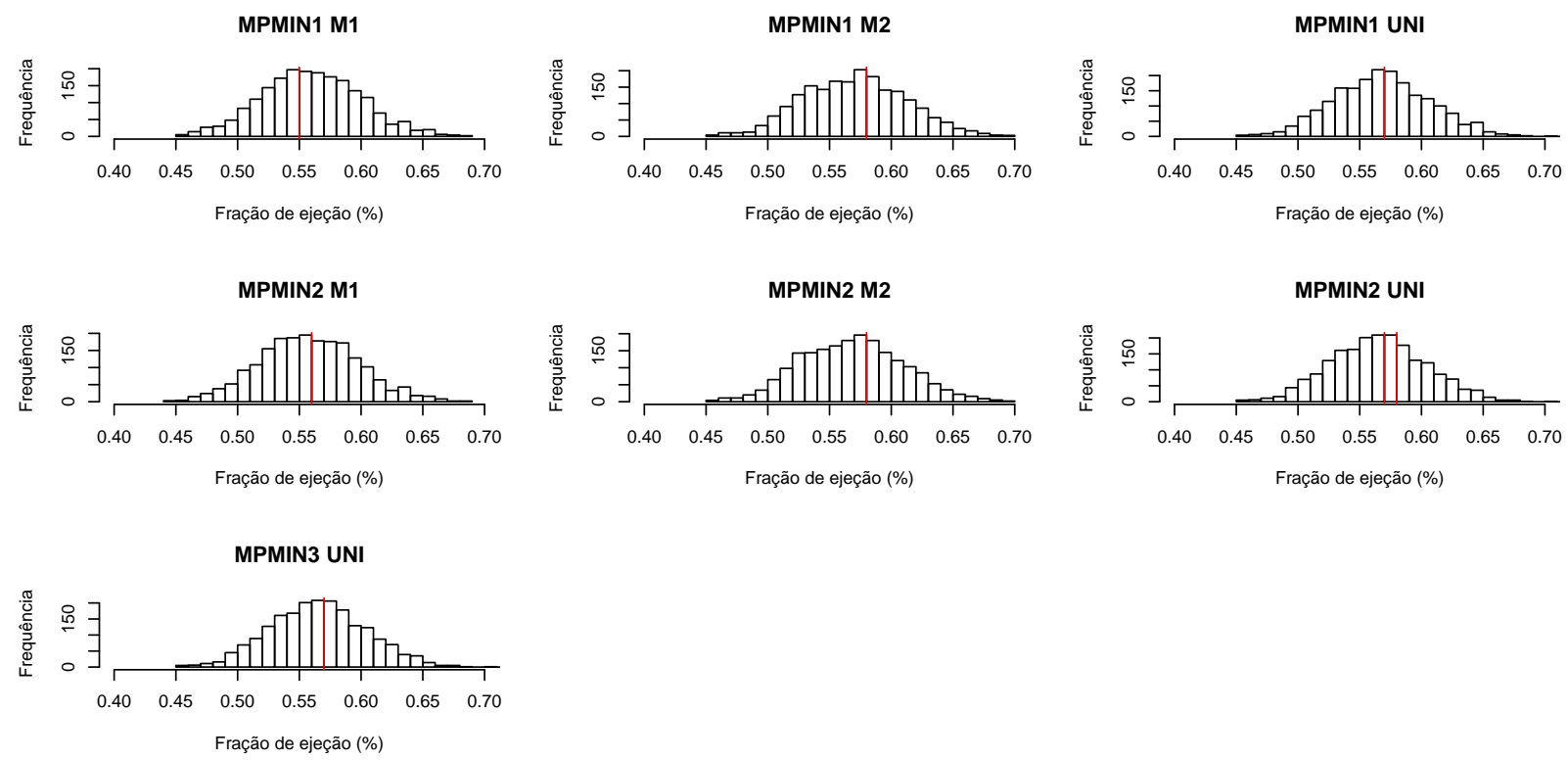

(b) Figura 2: Histogramas referentes aos riscos relativos estimados

Figura D.9: As figuras 1 e 2 apresentam os histogramas da distribuição dos pontos de corte $e$ riscos relativos estimados pelo método de validação bootstrap para os métodos de seleção de pontos de corte MPMIN1, MPMIN2 e MPMIN3 nos cenários M1, M2 e Uni, respectivamente. Linha vermelha indica a moda. (caso2) 

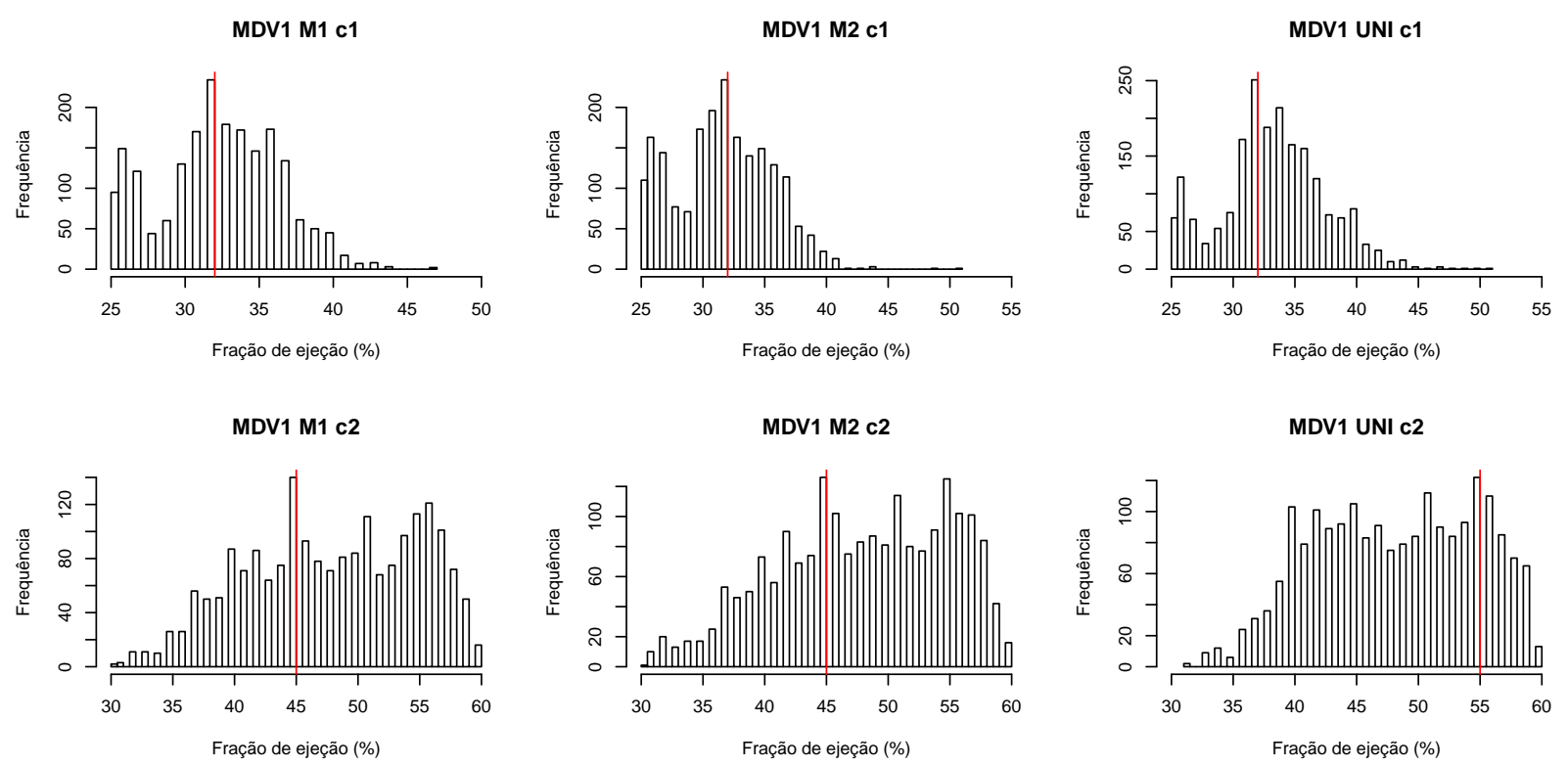

(a) Figura 1: Histogramas referentes aos pontos de corte c1 e c2 estimados
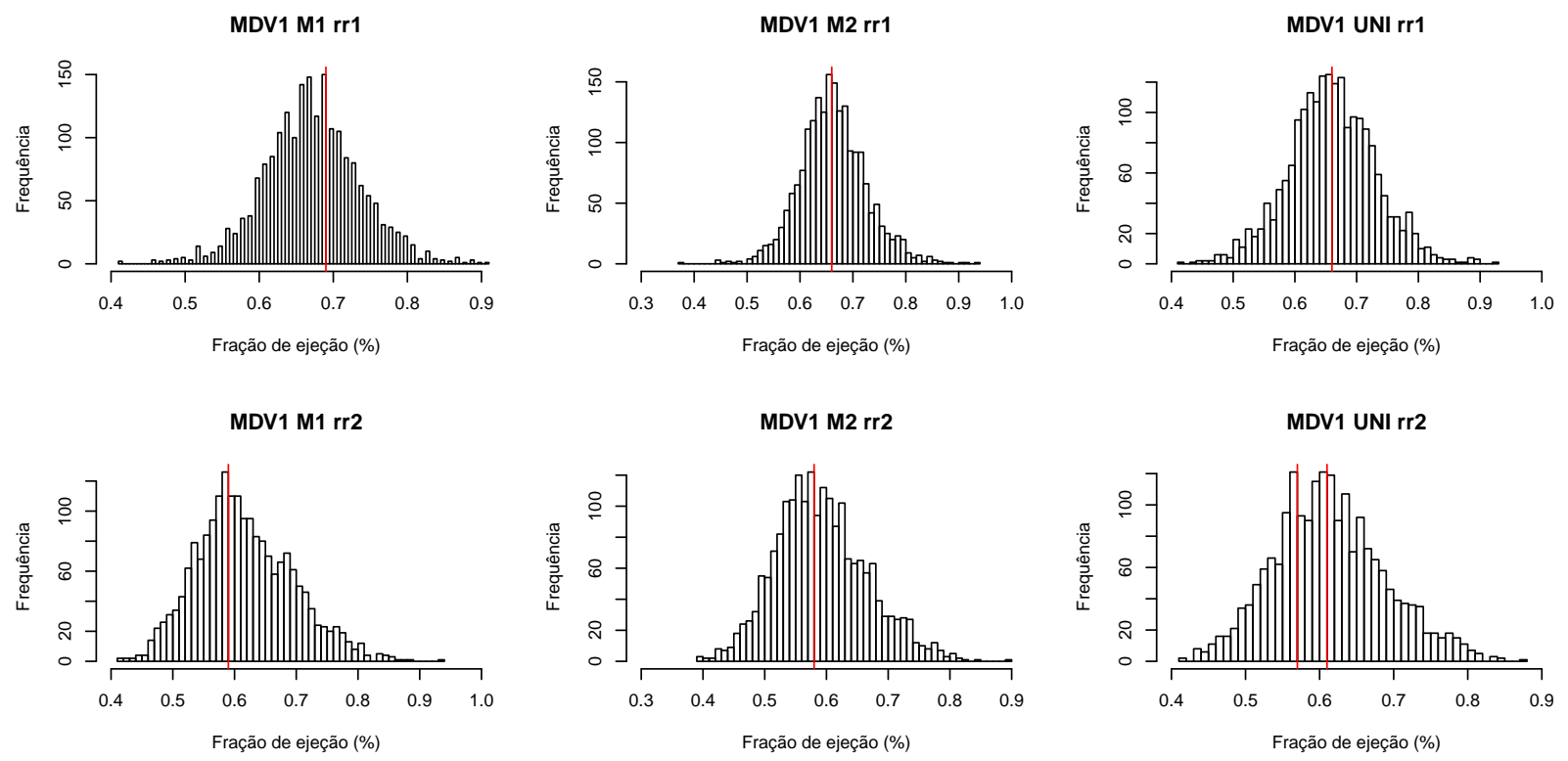

(b) Figura 2: Histogramas referentes aos riscos relativos rr1 e rr2 estimados

Figura D.10: As figuras 1 e 2 apresentam os histogramas da distribuição dos pontos de corte e riscos relativos estimados pelo método de validação bootstrap para o método de seleção de pontos de corte MDV1 nos cenários M1, M2, M3 e Uni, respectivamente. Linha vermelha indica a moda. (caso2) 
MDV2 M1 c1

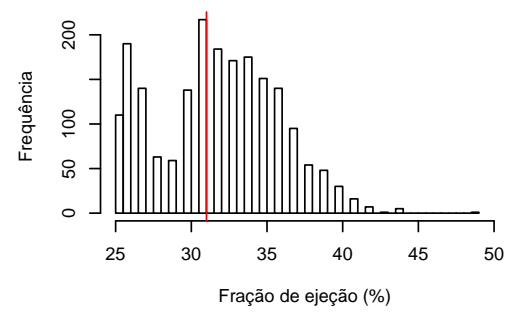

MDV2 M1 c2

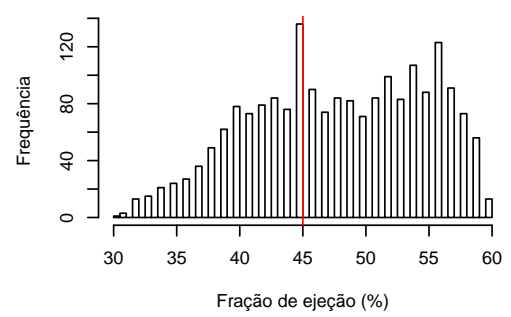

MDV2 M2 c1

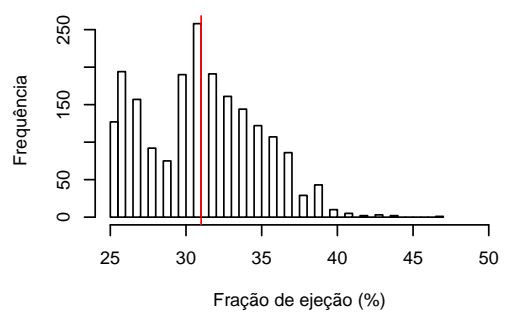

MDV2 M2 c2

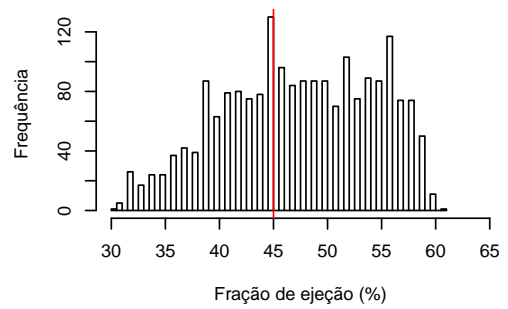

MDV2 UNI c1

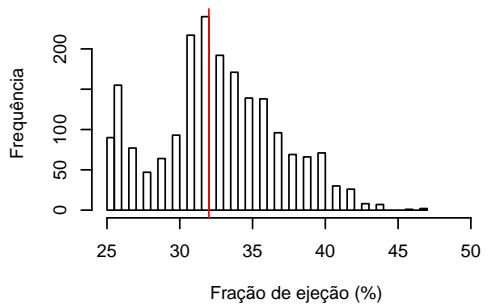

MDV2 UNI c2

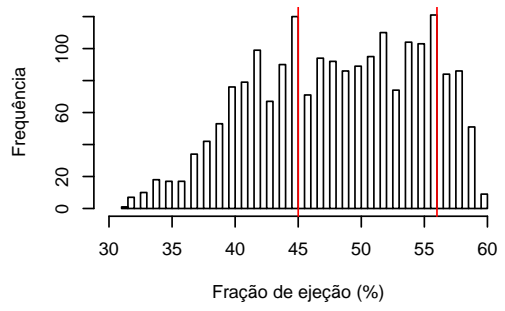

(a) Figura 1: Histogramas referentes aos pontos de corte c1 e c2 estimados
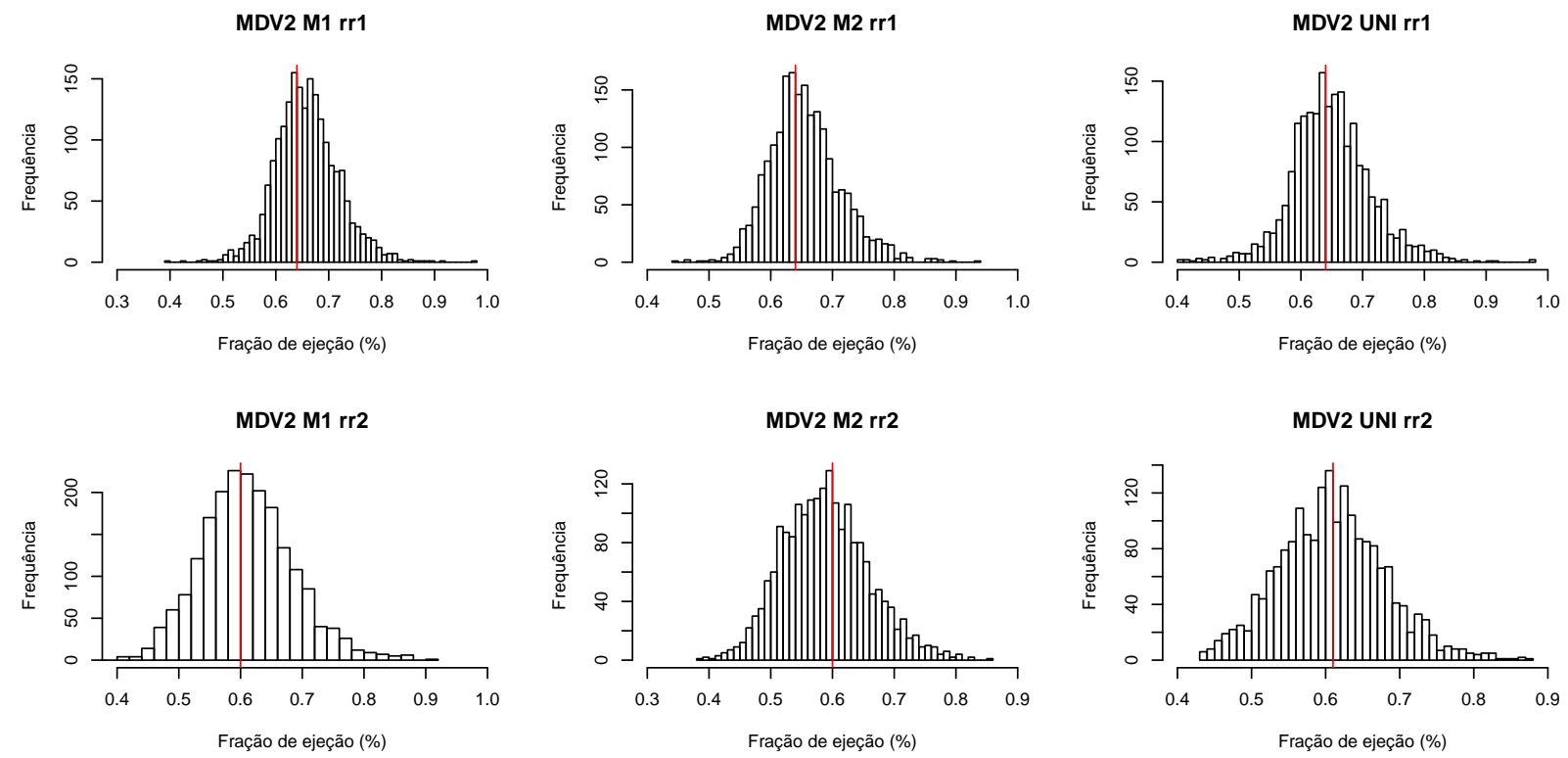

(b) Figura 2: Histogramas referentes aos riscos relativos rr1 e rr2 estimados

Figura D.11: As figuras 1 e 2 apresentam os histogramas da distribuição dos pontos de corte $e$ riscos relativos estimados pelo método de validação bootstrap para o método de seleção de pontos de corte MDV2 nos cenários M1, M2, M3 e Uni, respectivamente. Linha vermelha indica a moda. (caso2) 
MPMIN1 M1 c1

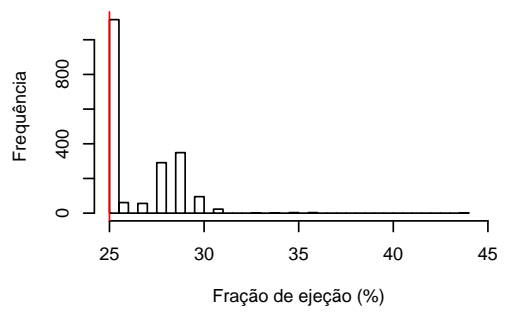

MPMIN1 M1 c2

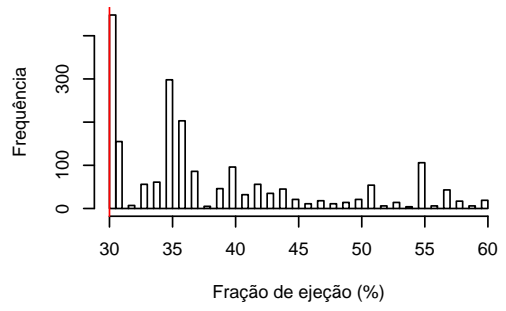

MPMIN1 M2 c1

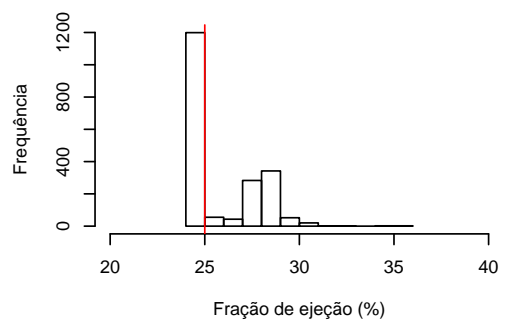

MPMIN1 M2 c2

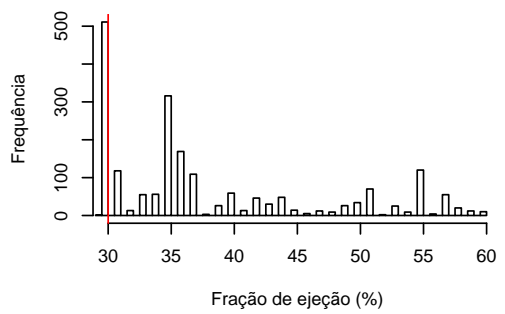

MPMIN1 UNI c1

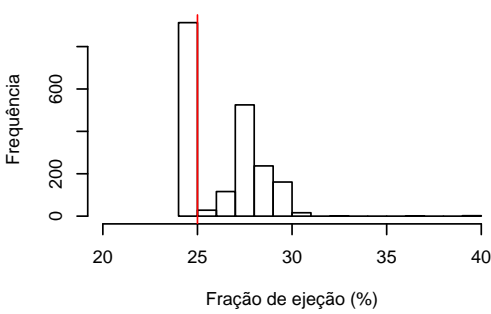

MPMIN1 UNI c2

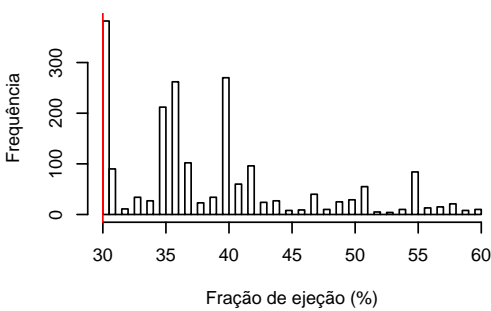

(a) Figura 1: Histogramas referentes aos pontos de corte c1 e c2 estimados

MPMIN1 M1 rr1

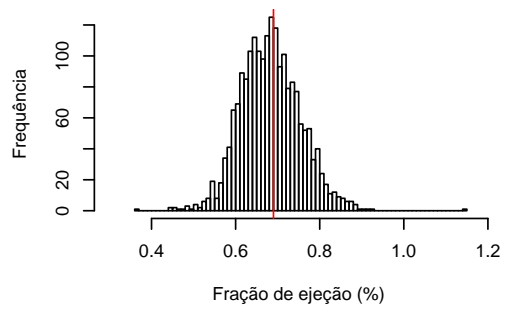

MPMIN1 M1 rr2

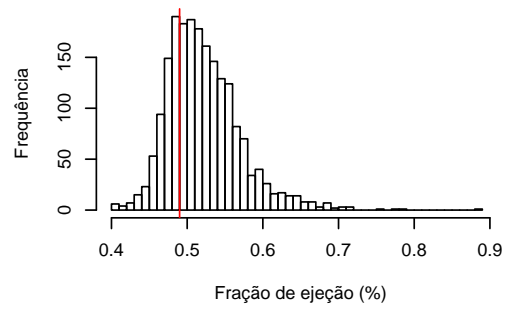

MPMIN1 M2 rr1

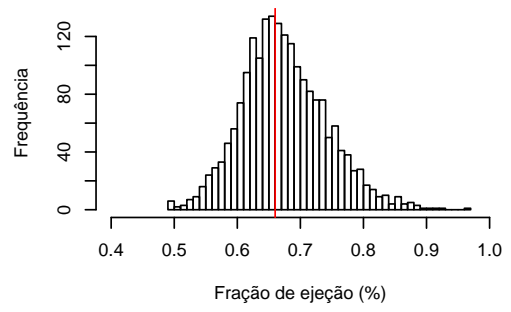

MPMIN1 M2 rr2

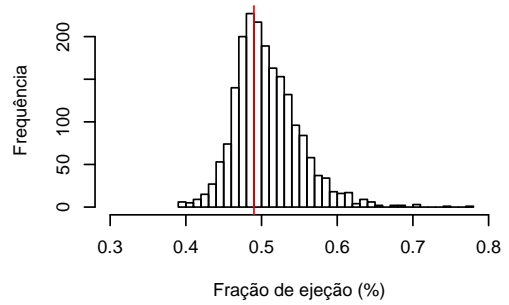

MPMIN1 UNI rr1

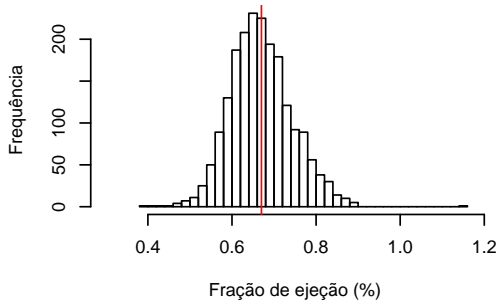

MPMIN1 UNI rr2

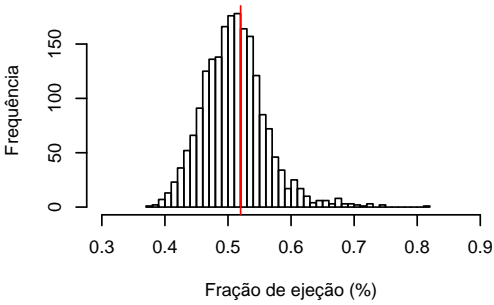

(b) Figura 2: Histogramas referentes aos riscos relativos rr1 e rr2 estimados

Figura D.12: As figuras 1 e 2 apresentam os histogramas da distribuição dos pontos de corte $e$ riscos relativos estimados pelo método de validação bootstrap para o método de seleção de pontos de corte MPMIN1 nos cenários M1, M2, M3 e Uni, respectivamente. Linha vermelha indica a moda. (caso2) 
MPMIN2 M1 c1

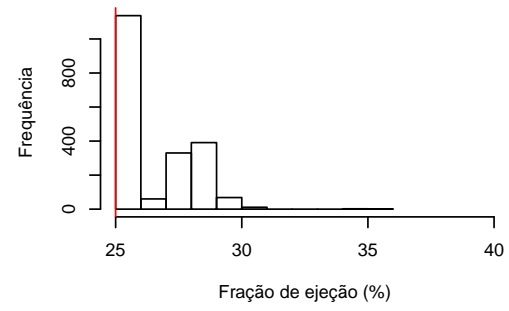

MPMIN2 M1 c2

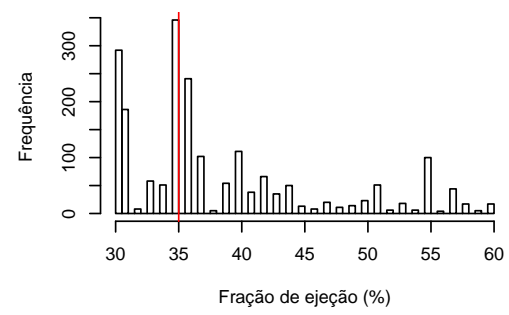

MPMIN2 M2 c1

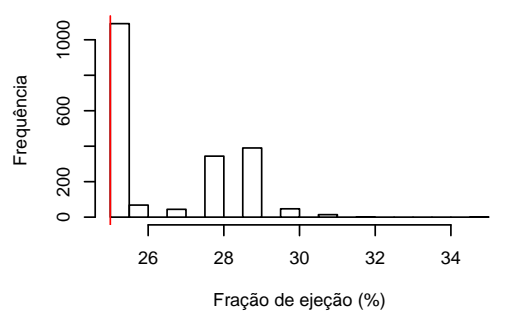

MPMIN2 M2 c2

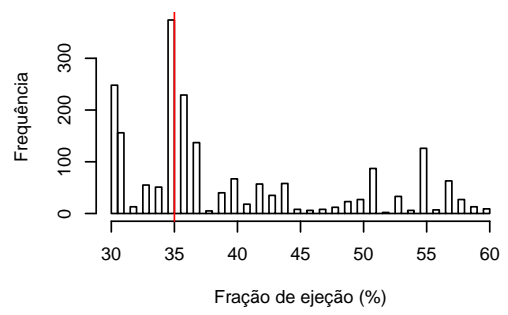

MPMIN2 UNI c1

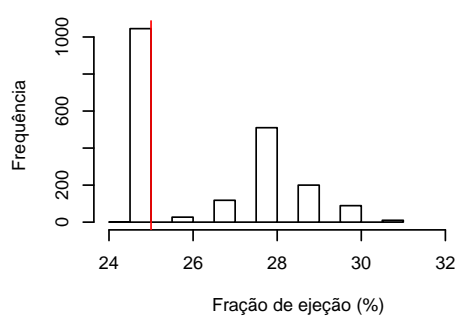

MPMIN2 UNI c2

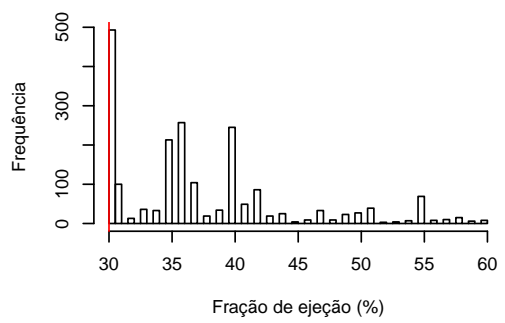

(a) Figura 1: Histogramas referentes aos pontos de corte c1 e c2 estimados

MPMIN2 M1 rr1

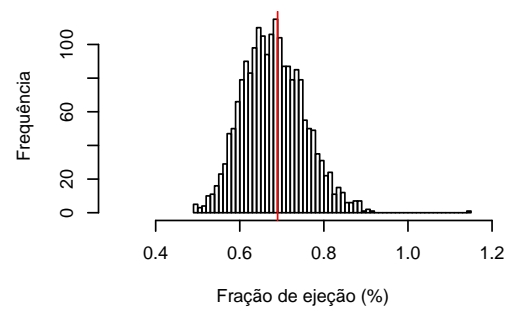

MPMIN2 M1 rr2

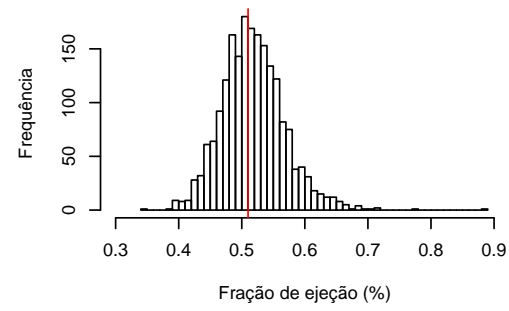

MPMIN2 M2 rr1

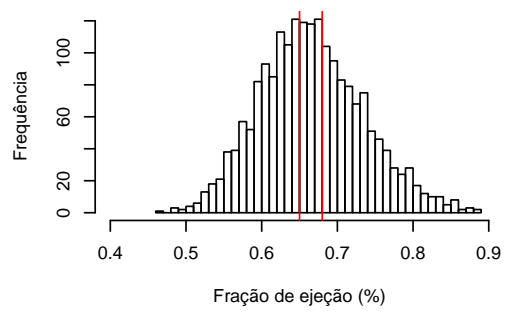

MPMIN2 M2 rr2

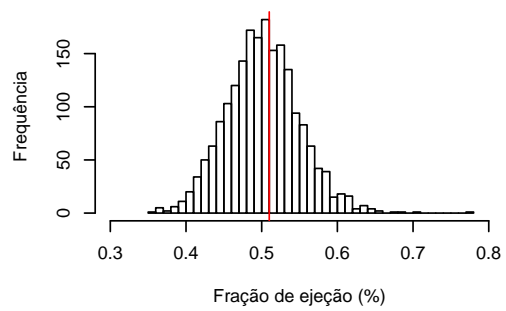

MPMIN2 UNI rr1

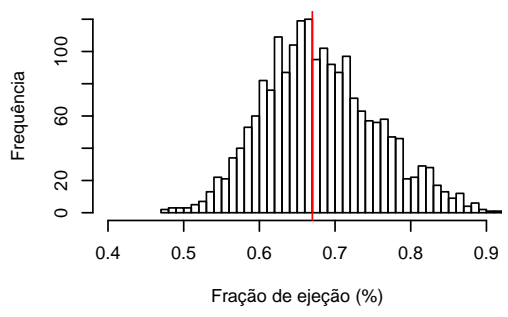

MPMIN2 UNI rr2

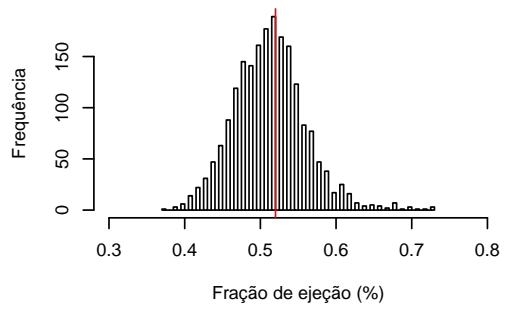

(b) Figura 2: Histogramas referentes aos riscos relativos rr1 e rr2 estimados

Figura D.13: As figuras 1 e 2 apresentam os histogramas da distribuição dos pontos de corte $e$ riscos relativos estimados pelo método de validação bootstrap para o método de seleção de pontos de corte MPMIN2 nos cenários M1, M2, M3 e Uni, respectivamente. Linha vermelha indica a moda. (caso2) 
MPMIN3 UNI c1

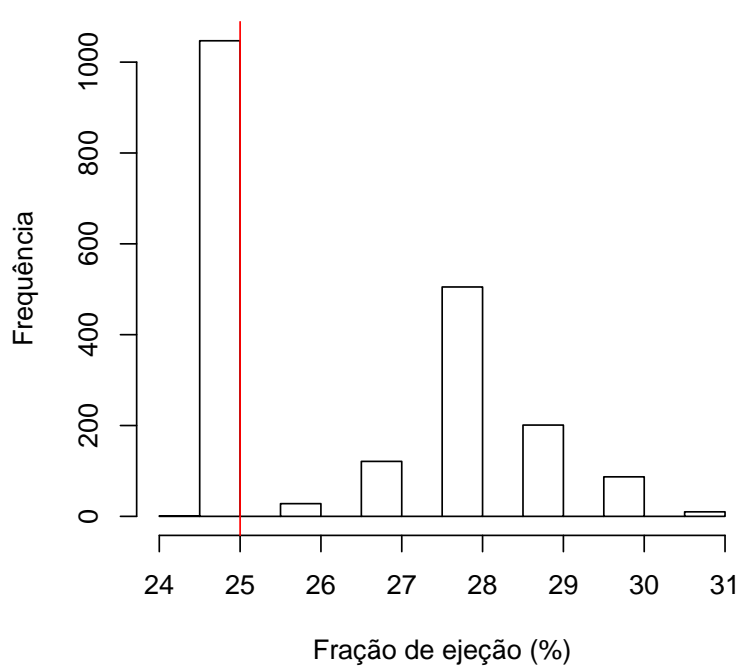

MPMIN3 UNI c2

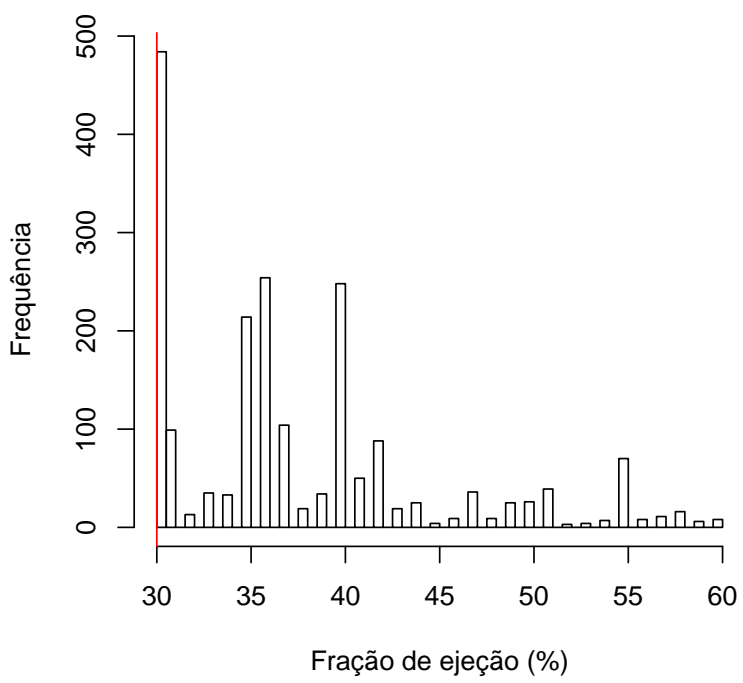

(a) Figura 1: Histogramas referentes aos pontos de corte c1 e c2 estimados

MPMIN3 UNI rr1

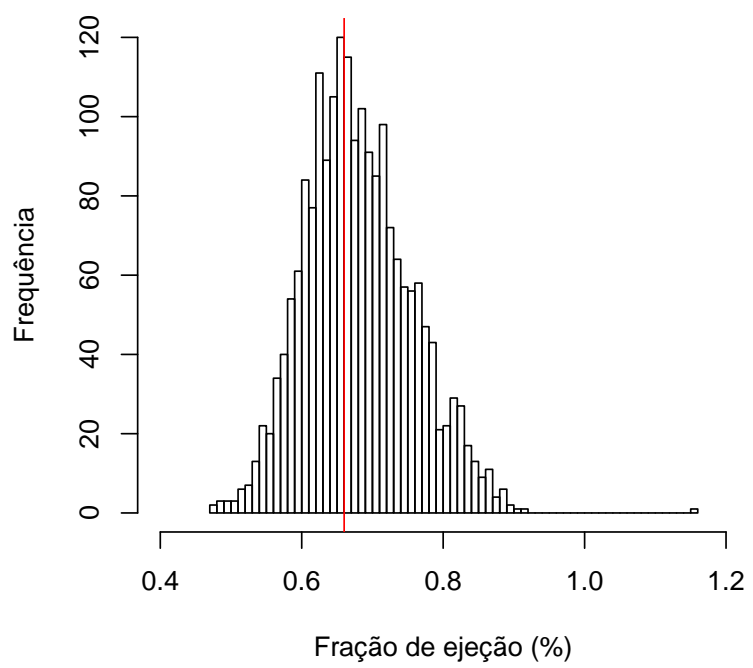

MPMIN3 UNI rr2

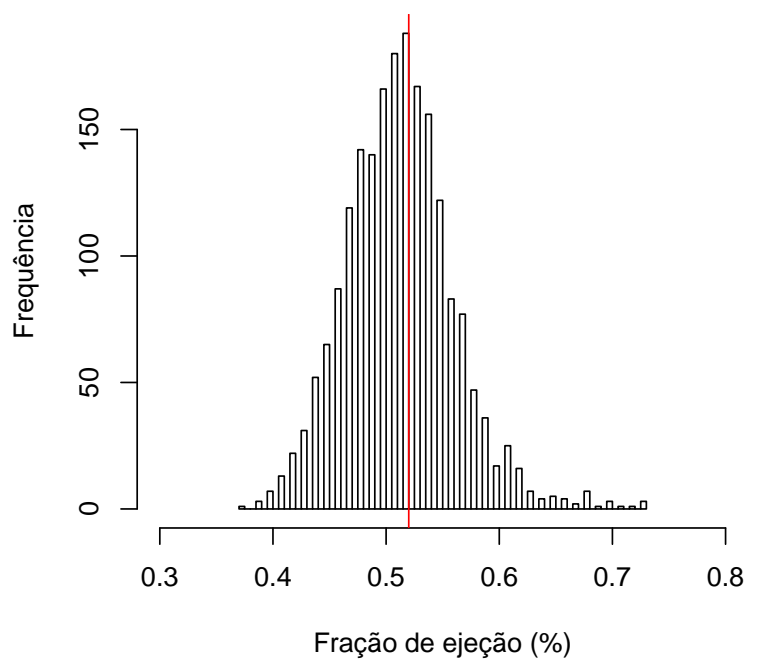

(b) Figura 2: Histogramas referentes aos riscos relativos rr1 e rr2 estimados

Figura D.14: As figuras 1 e 2 apresentam os histogramas da distribuição dos pontos de corte e riscos relativos estimados pelo método de validação bootstrap para o método de seleção de pontos de corte MPMIN3 no cenário Uni, respectivamente. Linha vermelha indica a moda. (caso2) 


\section{Referências Bibliográficas}

Altman(2005) Douglas G Altman. Categorizing continuous variable. Wiley Online Library. Citado na pág. 2, 13, 24, 25

Altman e Royston(2000) Douglas G. Altman e Patrick Royston. What do we mean by validating a prognostic model? Statistics in medicine, 19(4):453-473. Citado na pág. 1, 3, 35

Altman e Royston(2006) Douglas G Altman e Patrick Royston. The cost of dichotomising continuous variables. $B m j, 332(7549): 1080$. Citado na pág. 18, 19

Altman et al.(1994) Douglas G Altman, Berthold Lausen, Willi Sauerbrei e Martin Schumacher. Dangers of using "optimal" cutpoints in the evaluation of prognostic factors. Journal of the National Cancer Institute, 86(11):829-835. Citado na pág. 38, 39

Bennette e Vickers(2012) Caroline Bennette e Andrew Vickers. Against quantiles: categorization of continuous variables in epidemiologic research, and its discontents. $B M C$ medical research methodology, 12(1):1. Citado na pág. 2, 12, 18

Billingsley(1968) Patrick Billingsley. Convergence of probability measures. Wiley. Citado na pág. $27,31,37$

Bocchi et al.(2009) Edimar Alcides Bocchi, Fabiana Goulart Marcondes Braga, Silvia Moreira Ayub Ferreira, Luis Eduardo Paim Rohde, Wilson Alves de Oliveira, Dirceu Rodrigues de Almeida, Maria da Consolação Vieira Moreira, Reinaldo Bulgarelli Bestetti, Solange Bordignon, Clério Azevedo et al. III diretriz brasileira de insuficiência cardíaca crônica. Arquivos Brasileiros de Cardiologia, 93(1):3-70. Citado na pág. 6

Bocchi et al.(2012) Edimar Alcides Bocchi, FG Marcondes-Braga, Fernando Bacal, Almir Sérgio Ferraz, D Albuquerque e D Rodrigues. Atualização da diretriz brasileira de insuficiência cardíaca crônica-2012. Arquivos brasileiros de Cardiologia, 98(1):1-33. Citado na pág. 5

Bovitz et al.(2016) Tanya Bovitz, David T Gilbertson e Charles A Herzog. Administrative data and the philosopher's stone: Turning heart failure claims data into quantitative assessment of left ventricular ejection fraction. The American journal of medicine, 129(2): 223-225. Citado na pág. 5

Breslow(1972) Norman E Breslow. Discussion of professor cox's paper. J Royal Stat Soc $B, 34: 216-217$. Citado na pág. 17

Buettner et al.(1997) Petra Buettner, Claus Garbe e Irene Guggenmoos-Holzmann. Problems in defining cutoff points of continuous prognostic factors: example of tumor thickness in primary cutaneous melanoma. Journal of clinical epidemiology, 50(11):1201-1210. Citado na pág. 19,24 
Clark e Altman(2003) Taane G Clark e Douglas G Altman. Developing a prognostic model in the presence of missing data: an ovarian cancer case study. Journal of clinical epidemiology, 56(1):28-37. Citado na pág. 48

Clark et al.(2003) TG Clark, MJ Bradburn, SB Love e DG Altman. Survival analysis part iv: further concepts and methods in survival analysis. The British Journal of Cancer, 89 (5):781. Citado na pág. 13, 18, 25

Cohen(1983) Jacob Cohen. The cost of dichotomization. Applied psychological measurement, 7(3):249-253. Citado na pág. 13

Colosimo e Giolo(2006) Enrico Antônio Colosimo e Suely Ruiz Giolo. Análise de sobrevivência aplicada. Em ABE-Projeto Fisher. Edgard Blücher. Citado na pág. 1, 17, 22, 30, 39, 75

Connor(1972) Robert J Connor. Grouping for testing trends in categorical data. Journal of the American Statistical Association, 67(339):601-604. Citado na pág. 13

Contal e O'Quigley(1999) Cécile Contal e John O'Quigley. An application of changepoint methods in studying the effect of age on survival in breast cancer. Computational statistics Ef data analysis, 30(3):253-270. Citado na pág. 27, 28, 30, 31, 37, 38, 48, 53, 61

Dasgupta(1998) Anirban Dasgupta. Asymptotic relative efficiency (are). Encyclopedia of Biostatistics. Citado na pág. 79

David(1972) Cox R David. Regression models and life tables (with discussion). Journal of the Royal Statistical Society, 34:187-220. Citado na pág. 11

Efron e Tibshirani(1994) Bradley Efron e Robert J Tibshirani. An introduction to the bootstrap. CRC press. Citado na pág. 42

El Aouar et al.(2013) Lilia Maria Mameri El Aouar, Diana Meyerfreud, Pedro Magalhães, Sérgio Lamêgo Rodrigues, MP Baldo, Y Brasil et al. Relação entre volume do átrio esquerdo e disfunção diastólica em 500 casos de uma população brasileira. Arq Bras Cardiol, 101(1):52-8. Citado na pág. 5

Faraggi e Simon(1996) David Faraggi e Richard Simon. A simulation study of crossvalidation for selecting an optimal cutpoint in univariate survival analysis. Statistics in medicine, 15(20):2203-2213. Citado na pág. 3, 18, 20, 24, 38, 43

Freitas et al.(2005) Humberto FG Freitas, Paulo R Chizzola, Ângela T Paes, Antonio CP Lima e Alfredo J Mansur. Risk stratification in a brazilian hospital-based cohort of 1220 outpatients with heart failure: role of chagas' heart disease. International journal of cardiology, 102(2):239-247. Citado na pág. 5

Gill(1984) Richard D Gill. Understanding cox's regression model: a martingale approach. Journal of the American Statistical Association, 79(386):441-447. Citado na pág. 76

Gong(1986) Gail Gong. Cross-validation, the jackknife, and the bootstrap: excess error estimation in forward logistic regression. Journal of the American Statistical Association, 81(393):108-113. Citado na pág. 26

Heagerty e Zheng(2005) Patrick J Heagerty e Yingye Zheng. Survival model predictive accuracy and roc curves. Biometrics, 61(1):92-105. Citado na pág. 33 
Heller e Simonoff(1992) Glenn Heller e Jeffrey S. Simonoff. Prediction in censored survival data: a comparison of the proportional hazards and linear regression models. Biometrics, páginas 101-115. Citado na pág. 11, 17, 48, 80

Hilsenbeck e Clark(1996) Susan Galloway Hilsenbeck e Gary M Clark. Practical p-value adjustment for optimally selected cutpoints. Statistics in medicine, 15(1):103-112. Citado na pág. $3,19,36,38,39$

Holländer et al.(2004) Norbert Holländer, Willi Sauerbrei e Martin Schumacher. Confidence intervals for the effect of a prognostic factor after selection of an 'optimal'cutpoint. Statistics in medicine, 23(11):1701-1713. Citado na pág. 22, 38, 75

Hothorn e Lausen(2002) Torsten Hothorn e Berthold Lausen. Maximally selected rank statistics in r. $R$ News, 2(1):3-5. Citado na pág. 35, 36, 40

Hothorn e Lausen(2003) Torsten Hothorn e Berthold Lausen. On the exact distribution of maximally selected rank statistics. Computational Statistics $\&$ Data Analysis, 43(2): 121-137. Citado na pág. 36, 39

Hothorn e Zeileis(2008) Torsten Hothorn e Achim Zeileis. Generalized maximally selected statistics. Biometrics, 64(4):1263-1269. Citado na pág. 36, 40

Jespersen(1986) NCB Jespersen. Dichotomizing a continuous covariate in the Cox regression model. Københavns Universitet. Institute of Mathematical Statistics. Citado na pág. 27

Kalbfleisch e Prentice(2011) John D Kalbfleisch e Ross L Prentice. The statistical analysis of failure time data, volume 360. John Wiley \& Sons. Citado na pág. 76

Klein e Wu(2004) John P Klein e Jing-Tao Wu. Discretizing a continuous covariate in survival studies. Handbook of Statistics, 23:27-42. Citado na pág. 2, 19, 22, 26, 28, 35, 75

Klein et al.(2008) John P. Klein, Mette Gerster, Per Kragh Andersen e Sergey Tarima. Sas and $\mathrm{R}$ functions to compute pseudo-values for censored data regression. Computer methods and programs in biomedicine, 89(3):289-300. Citado na pág. 31

Koziol e Petkau(1978) James A Koziol e A John Petkau. Sequential testing of the equality of two survival distributions using the modified savage statistic. Biometrika, páginas 615623. Citado na pág. 28, 29

Lagakos(1988) SW Lagakos. Effects of mismodelling and mismeasuring explanatory variables on tests of their association with a response variable. Statistics in medicine, 7(1-2): 257-274. Citado na pág. 13

Lala et al.(2016) Radu Ioan Lala, Dan Darabantiu, Luminita Pilat e Maria Puschita. Galectina-3: Ligação entre rigidez miocárdica e arterial em pacientes com insuficiência cardíaca descompensada? Arq Bras Cardiol, 106(2):121-129. Citado na pág. 5

Lausen e Schumacher(1992) Berthold Lausen e Martin Schumacher. Maximally selected rank statistics. Biometrics, páginas 73-85. Citado na pág. 36, 37, 38

Lausen e Schumacher(1996) Berthold Lausen e Martin Schumacher. Evaluating the effect of optimized cutoff values in the assessment of prognostic factors. Computational Statistics \& Data Analysis, 21(3):307-326. Citado na pág. 18, 35 
Lausen et al.(1994) Berthold Lausen, Willi Sauerbrei e Martin Schumacher. Classification and regression trees (cart) used for the exploration of prognostic factors measured on different scales. Computational Statistics, páginas 483-496. Citado na pág. 39

Lawless(2011) Jerald F Lawless. Statistical models and methods for lifetime data, volume 362. John Wiley \& Sons. Citado na pág. 22, 23, 77

Lima e Saito(2015) Antonio Carlos Pedroso de Lima e Pedro Minoru Saito. Relatório de análise estatística sobre o projeto: "Análise dos pacientes portadores de insuficiência cardíaca com fração de ejeção preservada encaminhados para o hospital terciário de cardiologia". Relatório técnico, IME-USP, São Paulo. Citado na pág. 7

Maxwell e Delaney(1993) Scott E Maxwell e Harold D Delaney. Bivariate median splits and spurious statistical significance. Psychological bulletin, 113(1):181. Citado na pág. 12, 13, $18,25,26,48$

Mazumdar e Glassman(2000) Madhu Mazumdar e Jill R Glassman. Categorizing a prognostic variable: review of methods, code for easy implementation and applications to decision-making about cancer treatments. Statistics in medicine, 19(1):113-132. Citado na pág. $1,2,3,14,15,19,20,22,39,52$

Mazumdar et al.(2003) Madhu Mazumdar, Alex Smith e Jennifer Bacik. Methods for categorizing a prognostic variable in a multivariable setting. Statistics in medicine, 22(4): 559-571. Citado na pág. 2, 18, 19, 20, 41, 43, 44

Meijers et al.(2016) WC Meijers, AR van der Velde e RA de Boer. Biomarkers in heart failure with preserved ejection fraction. Netherlands Heart Journal, 24(4):252-258. Citado na pág. 5

Messias et al.(2016) Leandro Rocha Messias, Aryanne Guimarães Ferreira, Sandra Marina Ribeiro de Miranda, José Antônio Caldas, Jader Cunha de Azevedo Teixeira, Ana Carolina Nader Vasconcelos Messias, Elisabeth Maróstica e Claudio Tinoco Mesquita. Efeito do nebivolol sobre parâmetros do mibg e exercício na insuficiência cardíaca com fração de ejeção normal. Arq Bras Cardiol, 106(5):358-366. Citado na pág. 5, 6

Miller e Siegmund(1982) Rupert Miller e David Siegmund. Maximally selected chi square statistics. Biometrics, páginas 1011-1016. Citado na pág. 36, 37

Morgan e Elashoff(1986) Timothy M Morgan e Robert M Elashoff. Effect of categorizing a continuous covariate on the comparison of survival time. Journal of the American Statistical Association, 81(396):917-921. Citado na pág. 13

Moutinho et al.(2008) Marco Aurélio Esposito Moutinho, Flávio Augusto Colucci, Veronica Alcoforado, Leandro Reis Tavares, Mauricio Bastos Freitas Rachid, Maria Luisa Garcia Rosa, Mário Luiz Ribeiro, Rosemery Abdalah, Juliana Lago Garcia e Evandro Tinoco Mesquita. Insuficiência cardíaca com fração de ejeção preservada e com disfunção sistólica na comunidade. Arq Bras Cardiol, 90(2):145-50. Citado na pág. 6

Nakas et al.(2010) Christos T Nakas, Todd A Alonzo e Constantin T Yiannoutsos. Accuracy and cut-off point selection in three-class classification problems using a generalization of the youden index. Statistics in medicine, 29(28):2946-2955. Citado na pág. 33 
Nogueira et al.(2010) Patrícia Resende Nogueira, Salvador Rassi e Krislainy de Sousa Corrêa. Perfil epidemiológico, clínico e terapêutico da insuficiência cardíaca em hospital terciário. Arq Bras Cardiol, 95(3):392-8. Citado na pág. 5, 6

Paes et al.(2008) MJoelma Oliveira Paes, YAO Duarte, Maria Lúcia Lebrão, Jair Lício Ferreira Santos e Ruy Laurenti. Impacto do sedentarismo na incidência de doenças crônicas e incapacidades e na ocorrência de óbitos entre os idosos do município de são paulo. Saúde Coletiva, 5(24):183-8. Citado na pág. 1

Rota et al.(2015) Matteo Rota, Laura Antolini e Maria Grazia Valsecchi. Optimal cutpoint definition in biomarkers: the case of censored failure time outcome. BMC medical research methodology, 15(1):1. Citado na pág. 18, 32

Royston e Altman(1994) Patrick Royston e Douglas G Altman. Regression using fractional polynomials of continuous covariates: parsimonious parametric modelling. Applied statistics, páginas 429-467. Citado na pág. 12

Royston et al.(2006) Patrick Royston, Douglas G. Altman e Willi Sauerbrei. Dichotomizing continuous predictors in multiple regression: a bad idea. Statistics in medicine, 25(1):127141. Citado na pág. 2, 12, 19, 42, 43, 44

Ruopp et al.(2008) Marcus D Ruopp, Neil J Perkins, Brian W Whitcomb e Enrique F Schisterman. Youden index and optimal cut-point estimated from observations affected by a lower limit of detection. Biometrical Journal, 50(3):419-430. Citado na pág. 33

Santos e Vieira(2016) Eduardo Cavalcanti Lapa Santos e Jefferson Luís Vieira. Novas diretrizes para diagnóstico e tratamento da insuficiência cardíaca. https://www.pebmed.com.br/2016/07/13/ novas-diretrizes-para-diagnostico-e-tratamento-da-insuficiencia-cardiaca/, 2016. último acesso em 13/12/2016. Citado na pág. 6

Schumacher et al.(1997) Martin Schumacher, Norbert Holländer e Willi Sauerbrei. Resampling and cross-validation techniques: a tool to reduce bias caused by model building? Statistics in medicine, 16(24):2813-2827. Citado na pág. 19, 20, 24, 35, 40, 41, 42

Taylor e Yu(2002) Jeremy MG Taylor e Menggang Yu. Bias and efficiency loss due to categorizing an explanatory variable. Journal of Multivariate Analysis, 83(1):248-263. Citado na pág. 12, 14, 19

Tueller et al.(2016) Stephen J Tueller, Pascal R Deboeck e Richard A Van Dorn. Getting less of what you want: reductions in statistical power and increased bias when categorizing medication adherence data. Journal of behavioral medicine, páginas 1-12. Citado na pág. 18

Tunes-da-Silva e Klein(2011) Gisela Tunes-da-Silva e John P. Klein. Cutpoint selection for discretizing a continuous covariate for generalized estimating equations. Computational statistics \&̧ data analysis, 55(1):226-235. Citado na pág. 1, 31

Van Buuren et al.(1999) Stef Van Buuren, Hendriek C Boshuizen, Dick L Knook et al. Multiple imputation of missing blood pressure covariates in survival analysis. Statistics in medicine, 18(6):681-694. Citado na pág. 48

Van Houwelingen e Le Cessie(1990) JC Van Houwelingen e S Le Cessie. Predictive value of statistical models. Statistics in medicine, 9(11):1303-1325. Citado na pág. 42 
Verweij e Houwelingen(1993) Pierre J. M. Verweij e Hans C. Van Houwelingen. Crossvalidation in survival anlysis. Statistics in medicine, 12(24):2305-2314. Citado na pág. 41

Villacorta e Maisel(2016) Humberto Villacorta e Alan S Maisel. Teste com st2 solúvel: Um biomarcador promissor no tratamento da insuficiência cardíaca. Arq Bras Cardiol, 106(2):145-152. Citado na pág. 6

Vinh-Hung et al.(2009) Vincent Vinh-Hung, Helena M Verkooijen, Gerald Fioretta, Isabelle Neyroud-Caspar, Elisabetta Rapiti, Georges Vlastos, Carole Deglise, Massimo Usel, Jean-Michel Lutz e Christine Bouchardy. Lymph node ratio as an alternative to pn staging in node-positive breast cancer. Journal of clinical oncology, 27(7):1062-1068. Citado na pág. $25,26,35$

Weinberg(1995) Clarice R Weinberg. How bad is categorization? Epidemiology, páginas 345-347. Citado na pág. 12

White e Royston(2009) Ian R White e Patrick Royston. Imputing missing covariate values for the cox model. Statistics in medicine, 28(15):1982-1998. Citado na pág. 48

Williams et al.(2006) Brent A Williams et al. Finding optimal cutpoints for continuous covariates with binary and time-to-event outcomes. Relatório técnico, Citeseer. Citado na pág. 2,15

Worsley(1982) KJ Worsley. An improved bonferroni inequality and applications. Biometrika, 69(2):297-302. Citado na pág. 39 